\title{
Optimal Simple Money Rules in a Small Open Economy with Information Uncertainty and Measurement Error
}

\author{
By \\ Deming Luo \\ B.A. and M.A. Nanjing University, China \\ A thesis submitted to the Faculty of Graduate Studies and Research in partial \\ fulfillment of the requirement for the degree of \\ Doctor of Philosophy \\ In the Department of Economics \\ CARLETON UNIVERSITY \\ Ottawa, Ontario \\ November 2008
}

Copyright (C) 2008, Deming Luo

All rights reserved. This work may not be reproduced

in whole or in part, by photocopying or other means without

permission of the author 


$\begin{array}{ll}\begin{array}{l}\text { Library and } \\ \text { Archives Canada }\end{array} & \begin{array}{l}\text { Bibliothèque et } \\ \text { Archives Canada }\end{array} \\ \begin{array}{l}\text { Published Heritage } \\ \text { Branch }\end{array} & \begin{array}{l}\text { Direction du } \\ \text { Patrimoine de l'édition }\end{array} \\ \begin{array}{l}\text { 395 Wellington Street } \\ \text { Ottawa ON K1A 0N4 } \\ \text { Canada }\end{array} & \begin{array}{l}\text { 395, rue Wellington } \\ \text { Ottawa ON K1A 0N4 } \\ \text { Canada }\end{array}\end{array}$

Your file Votre référence ISBN: 978-0-494-47482-2

Ourfile Notre référence

ISBN: 978-0-494-47482-2

NOTICE:

The author has granted a nonexclusive license allowing Library and Archives Canada to reproduce, publish, archive, preserve, conserve, communicate to the public by telecommunication or on the Internet, loan, distribute and sell theses worldwide, for commercial or noncommercial purposes, in microform, paper, electronic and/or any other formats.

The author retains copyright ownership and moral rights in this thesis. Neither the thesis nor substantial extracts from it may be printed or otherwise reproduced without the author's permission.
AVIS:

L'auteur a accordé une licence non exclusive permettant à la Bibliothèque et Archives Canada de reproduire, publier, archiver, sauvegarder, conserver, transmettre au public par télécommunication ou par l'Internet, prêter, distribuer et vendre des thèses partout dans le monde, à des fins commerciales ou autres, sur support microforme, papier, électronique et/ou autres formats.

L'auteur conserve la propriété du droit d'auteur et des droits moraux qui protège cette thèse. $\mathrm{Ni}$ la thèse ni des extraits substantiels de celle-ci ne doivent être imprimés ou autrement reproduits sans son autorisation.
In compliance with the Canadian Privacy Act some supporting forms may have been removed from this thesis.

While these forms may be included in the document page count, their removal does not represent any loss of content from the thesis.
Conformément à la loi canadienne sur la protection de la vie privée, quelques formulaires secondaires ont été enlevés de cette thèse.

Bien que ces formulaires aient inclus dans la pagination, il n'y aura aucun contenu manquant.

\section{Canada}




\begin{abstract}
The central focus of this thesis is whether or not the exchange rate or some other dimension of the external side of the economy should form an integral part of the monetary rule of a small open economy (SOE) in which the central bank faces data deficiencies and individuals encounter measurement error. It does so in the context of a SOE model that features two of the most important empirical characteristics associated with SOEs-incomplete exchange rate pass-through and incomplete asset markets. After calibrating the implied dynamic stochastic general equilibrium (DSGE) model and deriving the loss function used by the monetary authority from the utility function of the representative household, I first examine what monetary policy rule would perform best if the monetary authority used the reaction coefficients that are typically adopted in the literature. Under a number of different information scenarios, the model's simulations suggest that the rule most often advocated for the closed economy case - the Taylor rulealso works best for this SOE. The thesis then goes on to consider whether the Taylor rule continues to perform best when the reaction coefficients in the money rule are derived optimally. For the same information cases as discussed earlier, the model's simulations now indicate that some reflection of the external environment facing the SOE-either the real exchange rate gap and/or the law of one price gap - always will improve monetary policy performance. The significance of this is not just that external factors should form a part of a money rule but that policy makers should be careful in simply using coefficients that have been found helpful in other parts of the literature.
\end{abstract}


In the course of this discovery, the thesis also uncovers a number of other important regularities, suggesting, for example, an important information role for interest rate smoothing. In addition, the welfare gain from including interest rate smoothing and/or the real exchange rate or law of one price gap in the policy rule increases more in those cases where the monetary authority has less current and/or less reliable information. Finally we find that the introduction of measurement error (for both private agents and the monetary authority) leaves the ranking of optimal money rules unaltered while introducing a new feature--the optimal weight placed on indicators that can be observed only with noise is now smaller than otherwise. 


\section{Acknowledgements}

After several years hard work, my thesis is finally complete. In looking back at the distance covered, I must first thank my supervisor, Professor J. Stephen Ferris, who has been a constant source of help, encouragement, and guidance. Through our years of association, our many long conversations have been invaluable in improving my understanding of macroeconomics, monetary economics and research generally. The experience of working with him will benefit me forever. Therefore I wish to express my sincere thanks for his contributions to the thesis: his time, patience, understanding, and affection.

I would also like to thank Professor Ehsan Choudhri, Professor Lilia Karnizova, Professor Yiagadeesen Samy, Professor Yazid Dissou. Over the past few years they have provided me with many constructive comments and suggestions that have greatly improved the coherence and structure of my thesis. Dr. Ali Dib of Bank of Canada, the external examiner, gave me many constructive comments and suggestions during my thesis examination, I would like to express my sincere thanks to him. I am also grateful to Professor Christopher Worswick who, as joint Ph.D. program supervisor, not only helped me with my program of study and research, but also provided generous financial support at critical intervals. This was especially appreciated.

I would also like to specifically thank Professors Michael J. Moore of the School of Mathematics and Statistics at Carleton University and Jiankang Zhang of the Department of Economics, for their help with the mathematics used to derive the thesis model. Somewhat more generally, I would also like to thank Professors Huntley 
Schaller, Hashmat Khan, Fanny S. Demers, Michel Demers, Charles Freedman, Makoto Nirei, Patrick J. Coe, P. N. Rowe, Zhiqi Chen and Zhihao Yu for offering advice and constructive criticism at various stages in my graduate program and during the writing of my thesis. Last, but not least, I would like to thank the Economics Department, from its Chair, Professor Keir Armstrong, through the Departmental Administrator, Ms. Dawn Bjornson, to the Graduate Administrator, Ms. Marge Brooks, and the rest of the staff for the environment and help that have left me with so many pleasant memories of my period of study and teaching here at Carleton University.

Finally, I cannot adequately express my appreciation to my wife, Dr. Lijan Hou, for her patience, understanding, and encouragement. In supporting my years of study and in raising my daughter Julia, she has born all the burdens of life and endured hardships that others would have found unendurable. For all this she has earned my unconditional love. I only hope I can make the results of my study worthy of her sacrifice. 


\section{Table of Contents}

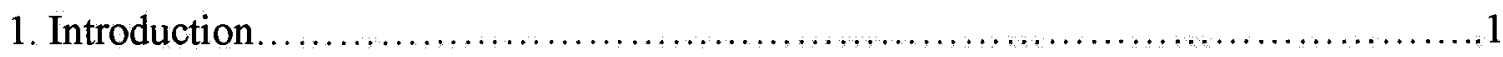

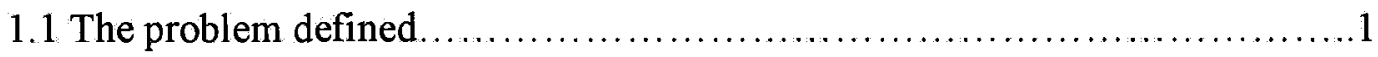

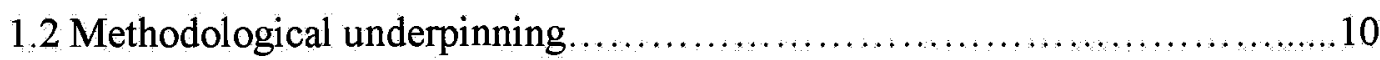

1.3 Basic features and contributions..................................... 12

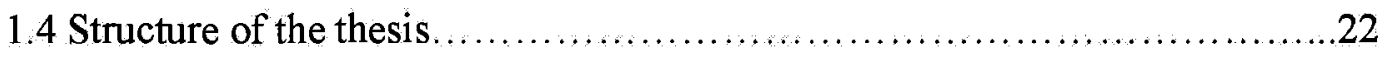

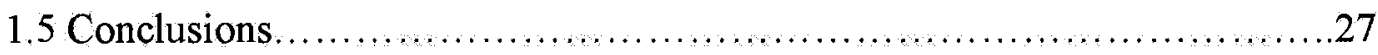

2. A small open economy model with incomplete exchange rate pass-through............30

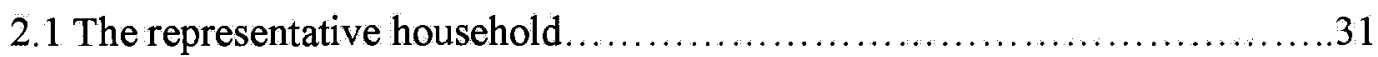

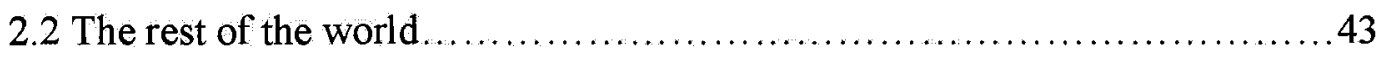

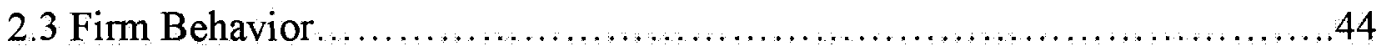

2.4 Import Pricing Behavior ...........................................49

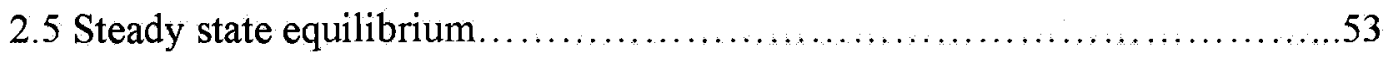

2.6 The new Keynesian Phillips curve ......................................58

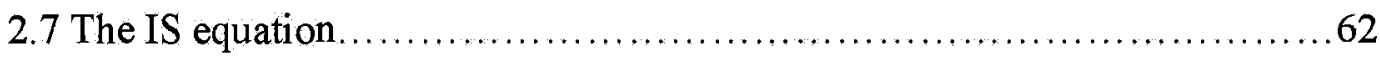

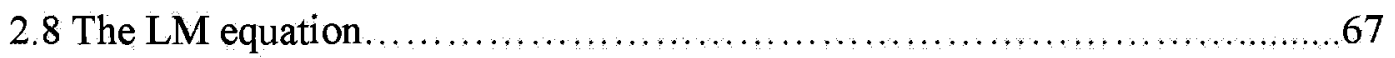

2.9 The evolution of the real exchange rate and net foreign assets.............68

2.10 Rewriting the equations of motion in terms of efficiency gaps............71

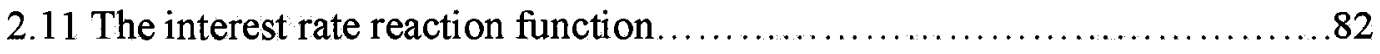

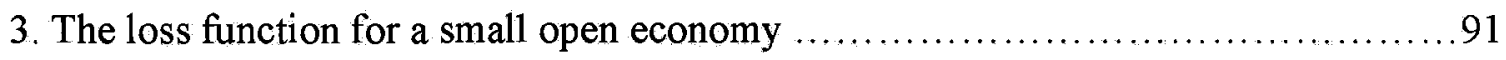

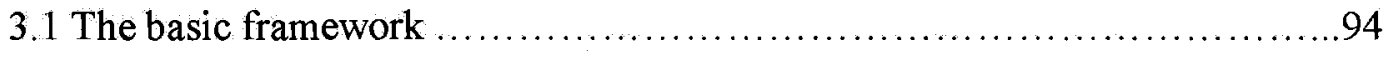

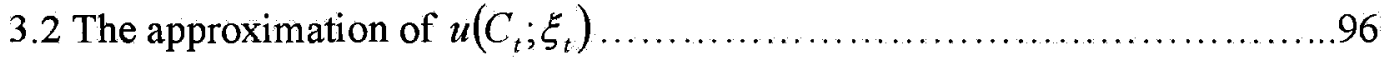


3.3 The approximation of $\int_{0}^{1} \widetilde{v}\left(y_{t}(i) ; \ddot{\xi}_{t}\right) d i$

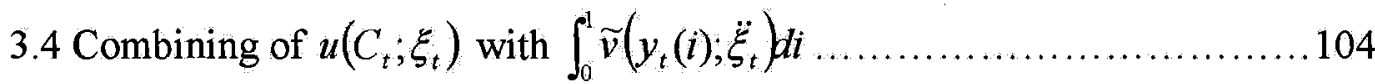

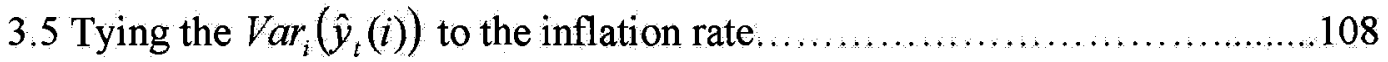

3.6 The period loss function without the real balances........................111

3.7 The period loss function with the real balances .........................116

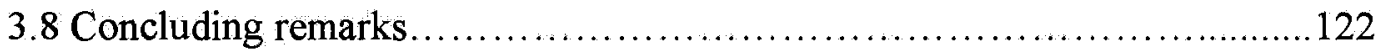

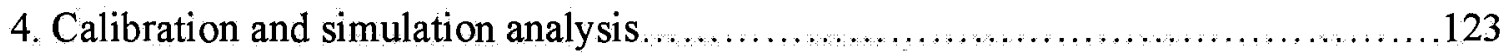

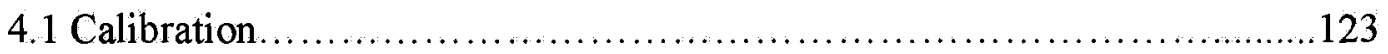

4.2 Impulse responses of key variables to shocks........................... 131

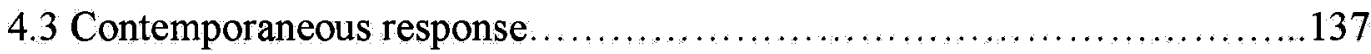

4.4 Data availability allows only lagged response ..........................152

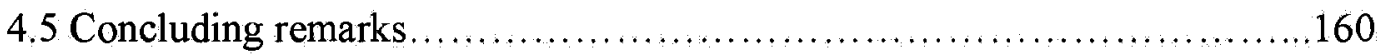

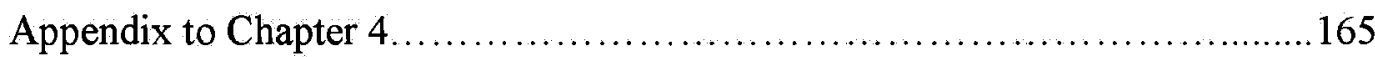

5. Fully optimal monetary policy rules for the small open economy..................170

5.1 The mathematics of optimal policy rules............................... 170

5.2 A fully optimal monetary policy rule under commitment....................174

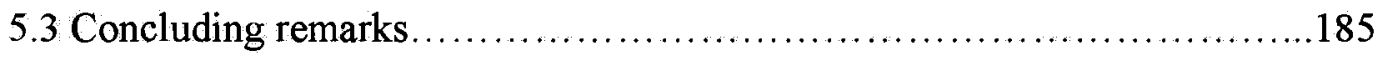

6. The role of openness considerations in optimal simple monetary policy rules........187

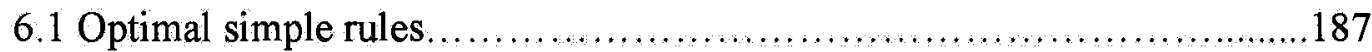

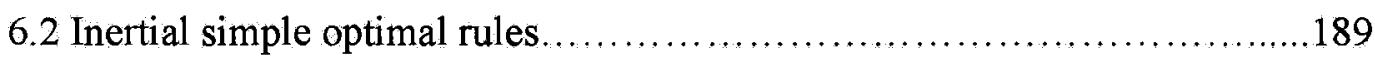

6.3 Optimal smoothing rules .......................................... 202

6.4 Non-inertial optimal simple rules....................................209 
6.5 Robustness analysis.

6.6 Concluding remarks

Appendix to Chapter 6

7. Optimal simple monetary policy rules when households observe aggregate prices with error. 235

7.1 Introduction 233

7.2 The model of a small open economy with private measurement error. 239

7.3 Optimal simple policy rules when private agents observe aggregate price levels with error 248

7.4 Optimal simple policy rules when both private agents and policymakers face the same measurement error. 259

7.5 Concluding remarks 267

References. .270 


\section{List of Tables}

Table 1.1 Inflation targeting in some countries.

Table 4.1 The parameter values chosen to calibrate the model......................127

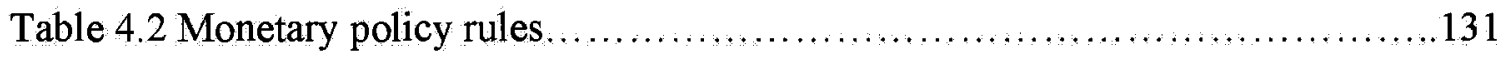

Table 4.3 Simple rules with domestic output prices in Case $1 \ldots \ldots \ldots \ldots \ldots \ldots \ldots \ldots . \ldots \ldots$

Table 4.4 Simple rules with interest rate smoothing in Case $1 \ldots \ldots \ldots \ldots \ldots \ldots \ldots \ldots 141$

Table 4.5 Inflation gaps measured in domestic output prices in Case 1 ...............143

Table 4.6 Domestic inflation gaps with interest-rate smoothing in Case $1 \ldots \ldots \ldots \ldots \ldots 143$

Table 4.7 The monetary authority responds to CPI inflation in Case $1 \ldots \ldots \ldots \ldots \ldots \ldots . . .145$

Table 4.8 CPI inflation and interest rate smoothing in Case $1 \ldots \ldots \ldots \ldots \ldots \ldots \ldots \ldots \ldots$

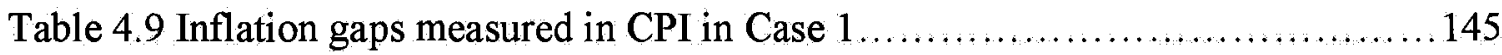

Table 4.10 CPI inflation gaps and interest rate smoothing in Case $1 . \ldots \ldots \ldots \ldots \ldots \ldots . \ldots 146$

Table 4.11 Targeting inflation measured in domestic prices in Case $2 \ldots \ldots \ldots \ldots \ldots \ldots . \ldots 151$

Table 4.12 Domestic prices and interest rate smoothing in Case $2 \ldots \ldots \ldots \ldots \ldots \ldots \ldots 152$

Table 4.13 Policy uses domestic output-price inflation in Case $3 \ldots \ldots \ldots \ldots \ldots \ldots \ldots \ldots 156$

Table 4.14 The domestic inflation with interest-rate smoothing in Case $3 \ldots \ldots \ldots \ldots \ldots . .157$

Table 4.15 Targeting the domestic output inflation gap in Case $3 \ldots \ldots \ldots \ldots \ldots \ldots \ldots \ldots . \ldots \ldots$

Table 4.16 Domestic inflation gap with interest rate smoothing in Case $3 \ldots \ldots \ldots \ldots \ldots .158$

Table 4.17 Inflation measured in domestic output prices in Case $4 \ldots \ldots \ldots \ldots \ldots \ldots \ldots$.........

Table 4.18 Domestic inflation with interest rate smoothing in Case $4 \ldots \ldots \ldots \ldots \ldots \ldots 160$

Table 6.1 Partially optimal simple rules and domestic prices in Case $1 \ldots \ldots \ldots \ldots \ldots \ldots . . .193$

Table 6.2 Partially optimal simple rules and CPI prices in Case $1 \ldots \ldots \ldots \ldots \ldots \ldots \ldots . \ldots 197$ 
Table 6.3 Partially optimal simple rules and domestic prices in Case 2.

Table 6.4 Partially optimal simple rules and domestic prices in Case $3 \ldots \ldots \ldots \ldots \ldots \ldots . . \ldots 1$

Table 6.5 Partially optimal simple rules and domestic prices in Case $4 \ldots \ldots \ldots \ldots \ldots \ldots . .201$

Table 6.6 Fully optimal simple rules and domestic prices in Case 1 .................204

Table 6.7 Fully optimal simple rules and domestic prices in Case $2, \ldots \ldots \ldots \ldots \ldots \ldots \ldots . \ldots 208$

Table 6.8 Fully optimal simple rules and domestic prices in Case $3 \ldots \ldots \ldots \ldots \ldots \ldots . \ldots 208$

Table 6.9 Fully optimal simple rules and domestic prices in Case $4 \ldots \ldots \ldots \ldots \ldots \ldots \ldots . .209$

Table 6.10 Non-inertial optimal simple rules and domestic prices in Case $1 \ldots \ldots \ldots \ldots .210$

Table 6.11 Non-inertial optimal simple rules and CPI prices in Case $1 \ldots \ldots \ldots \ldots \ldots \ldots .211$

Table 6.12 Non-inertial optimal simple rules and domestic prices in Case 2 ...........215

Table 6.13 Non-inertial optimal simple rules and domestic prices in Case $3 \ldots \ldots \ldots \ldots . .217$

Table 6.14 Non-inertial optimal simple rules and domestic prices in Case 4..........217

Table 6.15 The degree of exchange rate pass-through and CPI prices in Case $1 \ldots \ldots \ldots 222$

Table 6.16 The degree of exchange rate pass-through and domestic prices in Case 1..222

Table 6.17 The size of the risk premium shock and CPI prices in Case $1 \ldots \ldots \ldots \ldots \ldots \ldots .224$

Table 6.18 The size of the risk premium shock and domestic prices in Case 1 .........224

Table 6.19 The welfare gains from incorporating the lagged interest rate $\ldots \ldots \ldots \ldots \ldots . .227$

Table 7.1 Non-inertial optimal policy rules when inflation contains noise ............250

Table 7.2 Non-Inertial optimal policy rules when information is complete............251

Table 7.3 Partially optimal inertial policy rules when inflation contains noise $\ldots \ldots \ldots \ldots . .252$

Table 7.4 Partially optimal inertial policy rules when information is complete.........253

Table 7.5 Fully optimal inertial policy rules when inflation contains noise............255

Table 7.6 Fully optimal inertial policy rules when information is complete ............255 
Table 7.7 Non-inertial rules when inflation is observed with larger noise.............257

Table 7.8 Partially optimal inertial policy rules when inflation contains more noise....257

Table 7.9 Fully optimal inertial policy rules when inflation contains more noise.......257

Table 7.10 Non-inertial optimal policy rules when information is symmetric..........261

Table 7.11 Partially inertial rules when measurement error is symmetric .............261

Table 7.12 Fully optimal inertial rules when measurement error is symmetric.........262 


\section{List of Figures}

Figure 1,1 The output gap..........,

Figure 4.1 Impulse responses to a productivity shock ........................... 133

Figure 4.2 Impulse responses to a risk premium shock ......................... 135

Figure 7.1 Lagged interest rate serves to minimize the welfare loss in percentage ......253

Figure 7.2 Optimal coefficient values for inflation in non-inertial policy rules with and

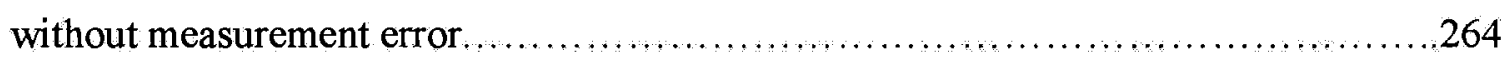

Figure 7.3 Optimal coefficient values for inflation in partially inertial policy rules with

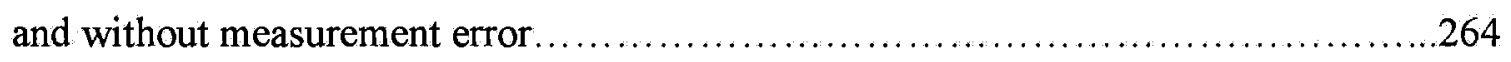

Figure 7.4 Fully optimal inflation coefficients with interest rate smoothing, with and

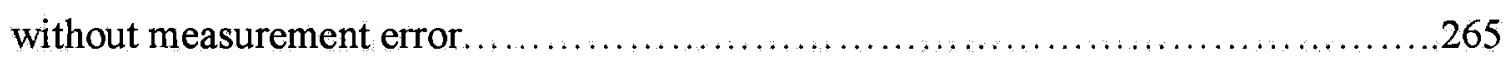

Figure 7.5 Optimized reaction coefficients of the Wicksellian natural rate for non-inertial policy rules. 267 


\section{Chapter 1}

\section{Introduction}

\subsection{The Problem Defined}

In this thesis I am interested in the properties of optimal simple monetary policy rules that can be adopted by a small open economy. Hence I begin by defining what is meant by a simple policy rule. A simple policy rule is a feedback rule in which a policy instrument, such as a short-term interest rate, is made to respond linearly to a set of endogenous variables, such as the deviation of inflation from its targeted value or the output gap. In a now classic paper, Taylor (1993) proposed that US monetary policy, during the period 1987 - 1992 of relatively stable inflation and growth rates, should be characterized by one such rule, in particular,

$$
i_{t}=i^{*}+\pi_{t}+0.5\left(\pi_{t}-\pi^{*}\right)+0.5 y_{t},
$$

where $i_{t}$ represents the U.S. federal funds rate, the intercept $i^{*}$ denotes the "equilibrium" real funds rate (set equal to 2 percent), $\pi_{t}$ is the rate of inflation over the previous four quarters, $\pi^{*}$ is the target inflation rate (set equal to 2 percent), and $y_{t}$ is the percent deviation of real GDP from its target. The rule implies that if both the inflation rate and the real GDP are on target, the federal funds rate would equal 4 percent, or 2 percent in real terms. Otherwise the federal funds rate would be raised when inflation exceeded its target and in the presence of a real output gap. Equation (1.1) soon became well known as 'the Taylor rule'. Following Taylor's (1993) pioneering work, a large and growing 
literature has developed on the relevance of the Taylor and other simple policy rules (see Taylor 1999, for example). A large number of studies in monetary policy now show that simple feedback money rules, especially of the Taylor type, work well in simulated economic models to deliver low inflation rates and output stability in the presence of monetary and real shocks. Similarly, many empirical studies report that Taylor policy specifications can describe the actual behavior of central banks in several countries. ${ }^{1}$

Perhaps because of its original application to the U.S. economy, the analyses of alternative simple policy rules were typically undertaken in a closed economy context. However, it wasn't long before economists began to ask whether a Taylor type monetary policy rule would still be efficient as a guideline for conducting monetary policy in an open economy setting. In the small open economy literature, a number of simple monetary policy rules have been investigated in a number of different modeling contexts, but a consensus conclusion has not yet been reached. That is, the literature has not yet resolved whether the Taylor rule is a desirable monetary policy rule for an open economy and/or whether the exchange rate should form part of the rule. Should the latter prove positive, a further unresolved issue is how should the policy instrument change in response to the exchange rate?

In surveying the current open economy literature on monetary policy, the following papers are illustrative of the wide variety of different conclusions that have been offered with respect to different policy rules. For example, using a backwardlooking open economy model with sticky prices, Ball (1999) argues that a money rule that includes the real exchange rate will be optimal when compared to either simple

\footnotetext{
${ }^{1}$ See, for example, Rotemberg and Woodford (1999), Rudebusch and Svensson (1999), Clarida, Gali and Gertler $(1998,2000)$.
} 
inflation targeting or the use of the Taylor rule, unless the latter are modified in important ways. ${ }^{2}$ In a dynamic stochastic forward-looking general equilibrium model with the incomplete exchange rate pass-through, Monacelli (2003) finds that optimal commitment (relative to discretion) will entail smoothing deviations from the law of one price and hence requires more stable nominal and real exchange rates. Using a two-country model, Benigno and Benigno (2000) and Weerapana (2000) report substantial (world) welfare improvement when an exchange rate term is incorporated into the policy rule. Similarly, Smets and Wouters (2002) argue that an exclusive focus on the stabilization of domestic price inflation is no longer optimal when import prices are sticky and exchange rate passthrough is gradual and Malik (2005) demonstrates that the welfare-enhancing policy implies a "dirty floating" under domestic inflation targeting when incomplete and imperfect asset markets are introduced to the model.

On the other hand, Clarida, Gali, and Gertler (2001), Aoki (2001), Gali and Monacelli (2002), find that the policy problem for the small open economy in which the households are allowed to share consumption risk internationally and exchange rate passthrough is complete is essentially identical to the policy problem faced by the closed economy. Hence the closed economy rule will remain optimal if combined with flexible exchange rates. A similar result is obtained by Clarida, Gali, and Gertler (2002) when the analysis is extended to a two-country setting. They conclude that central banks need only adjust the interest rate in response to domestic inflation when the economy is characterized by a Nash equilibrium, while under a cooperative equilibrium, interest rates should respond to foreign inflation as well. In either case, flexible exchange rates are

\footnotetext{
${ }^{2}$ For robustness, Ball (1999) considers the six rules, distinguished mainly by the size of their reaction coefficients and lagged interest rate terms.
} 
desirable. Moreover, when exchange rate pass-through is incomplete, Dib (2003) shows that the optimal policy problem for a small open economy is isomorphic to that for a closed economy, but Kollmann (2002), Batini, Harrison, and Millard (2003), Leitemo and Soderstrom (2005), and Adolfson (2007) all find that welfare performance is improved only marginally by incorporating an exchange rate term into the policy rule. Using a multi-country model with complete exchange rate pass-through, Taylor (1999) simulates a policy rule for the European Central Bank interest rate that includes the exchange rate as well as output and inflation, and finds that the policy outcomes are mixed. In some countries (France and Italy), a policy rule that includes the exchange rate leads to better performance, but in others (Germany) produces poorer performance. In closely related work, Devereux and Engel (2003) show that the impact of alternative exchange rate regimes on the policy performance is closely related to the pricing strategy employed in setting prices of import goods. In particular, when prices are pre-set in the currency of the producer (denoted producer currency pricing, PCP), floating exchange rates are optimal in the presence of country specific real shocks. However, when prices are pre-set in the consumer's currency (denoted local currency pricing, LCP), there is no benefit to exchange rate flexibility. In a small emerging economy, Choudhri (2005) finds that the relative performance of the alternative rules depends on the social welfare criterion used for assessing different policy rules and on the type of shock that the economy experiences. $^{3}$

\footnotetext{
${ }^{3}$ Choudhri argues that the loss function used to evaluate different monetary policy rules should also include exchange rate variability if there is fear of a floating exchange rate within the emerging economy. Variability of the interest rate may also be of concern, especially if financial institutions are vulnerable. However the traditional criterion does not include these terms.
} 
In this thesis I evaluate the relative performance of a series of different simple monetary policy rules applied to a small open economy. The objective is to discover a set of regularities that describe the optimal properties or characteristics of a simple optimal money rule for a small open economy in which the central bank faces data information uncertainty. The small open economy developed here is distinctive in that it incorporates Calvo pricing with indexation (to induce sticky domestic prices) together with incomplete exchange rate pass-through (because of quadratic costs of import price adjustment) and finally incomplete asset markets. With a domestic economy of this type and facing a number of domestic and external shocks, the economy is assumed to have a welfare maximizing central bank that can choose across a number of potential money rules for interest rate setting. Five simple rules are considered both with and without interest rate smoothing. Rule 1 is the most comprehensive rule, corresponding to the case where the monetary authority sets the short-term interest rate in response to changes in the inflation rate, the real GDP output gap, the real exchange rate gap, and the law of one price gap. This we call the comprehensive rule (C). Dropping the law of one price gap from Rule 1 gives us Rule 2, called the real exchange rate rule (ER). For comparative purposes, we replace the real exchange rate term in Rule 2 with the law of one price gap. This becomes Rule 3, the exchange rate pass-through rule (PT). Rule 4 has no openness component and corresponds to the Taylor rule (TR). Rule 5 further supposes that the monetary policy authority chooses to focus exclusively on inflation. This we call inflation targeting (IT). ${ }^{4}$

\footnotetext{
${ }^{4}$ The terminology used here differs somewhat from that used by Svensson (2000). Svensson links "targeting" to the monetary authority's objective. Hence when stabilizing inflation around the inflation rate target is the only objective for monetary policy, Svensson calls this strict inflation targeting. When there are additional objectives for monetary policy, for instance stabilizing output as well, monetary policy is called flexible inflation targeting.
} 
As suggested above, an important policy issue in which I am interested is what has been called policy inertia. For a closed economy, Aoki (2003) has shown that optimal policy will involve the lagged interest rate, where the degree of interest-rate smoothing depends on the degree of demand shock persistence. In addition, actual monetary policy in the United States and elsewhere seems to involve some degree of interest-rate smoothing. For example, Clarida et al (2000) estimate the degree of interest-rate smoothing at 0.68 for pre-Volcker period, and 0.79 for Volcker-Greenspan era. Across six different quarterly U.S. data samples (differing in their definition of output gap), Kozicki (1999) reports a range for the degree of interest-rate smoothing is from 0.75 to 0.82 , while across 16 different quarterly samples of U.S. data (differing in output gap, inflation, and sample period definition), Amato and Laubach (1999) report a range of the degree of interest-rate smoothing from 0.78 to 0.92 . Since most of studies are based on a closed model of a (largely) closed economy, it is of interest to ask whether optimal monetary policy will still involve interest rate smoothing when the economy is open.

A third issue addressed in the thesis is which measure of inflation should be the target of the policy maker. In a closed economy there will be no difference between domestic output and consumer price inflation. Hence it won't matter whether the central bank targets inflation measured in terms of the GDP deflator, the producer price index (PPI), or the consumer price index (CPI). However, because in an open economy the CPI includes import as well as domestic output prices, the answer is not straightforward. In practice, it appears that most countries that have adopted explicit inflation-targeting have targeted CPI inflation (or a close variant). Table 1.1 presents evidence of current practice. In academic policy research, however, Clarida et al. (2001) and Aoki (2001) have argued 
that it is domestic output price inflation that should be targeted, while Adolfson (2007) argues that policy in response to CPI inflation will be optimal. Finally, Huang and Liu (2005) find that a hybrid rule under which the short-term interest rate responds to the CPI inflation and PPI inflation results in a welfare level close to the optimum, whereas policy rules ignore PPI inflation can result in a significant welfare loss. In this thesis I will reexamine this issue at length.

Table 1.1 Inflation targeting in some countries

\begin{tabular}{l|l|l}
\hline Countries & Year adopted & Target variable \\
\hline Australia & 1993 & CPI (after 1988) \\
\hline Canada & 1991 & CPI (excluding food, energy and taxes) \\
\hline Finland & 1993 & CPI (excluding taxes, housing, and interest) \\
\hline Israel & 1991 & CPI \\
\hline New Zealand & 1988 & CPI (excluding taxes and interest) \\
\hline Spain & 1994 & CPI \\
\hline Sweden & 1993 & CPI \\
\hline UK & 1992 & Retail price index (excluding interest) \\
\hline
\end{tabular}

Source: Bernanke et al. (1999), Huang and Liu (2005).

A fourth issue considered is what should be the relevant criteria for measuring the relative performance of different simple policy rules; that is, what is the appropriate loss function? In the current literature, the standard criterion employed to evaluate monetary policy is to assume a loss function that is an increasing function of the levels of variance in inflation and the output gap. ${ }^{5}$ Often this is given the form, $L_{t}=\hat{\Pi}_{t}{ }^{2}+\lambda x_{t}{ }^{2}$,

\footnotetext{
${ }^{5}$ Corsetti and Pesenti (2005) argue that the policy objective can be written in an equivalent form as deviations from the levels arising under flexible prices. A similar welfare criterion is also adopted by Choudhri (2005).
} 
where the parameter $\lambda \geq 0$ denotes the relative weight that the central bank places on output stabilization and inflation is measured in terms of domestic output prices (see Aoki, 2003, for example). When the analysis is extended to an open economy, inflation is often measured by the CPI (see Monacelli, 2003, and Adolfson, 2007).

In relation to this loss function; several questions can be raised. First of all, what is the appropriate value to set for the parameter $\lambda$. In the literature, $\lambda$ is often set equal to 0.5 . While such a specification is plausible, with the value of $\lambda$ assigned arbitrarily, it is ultimately ad hoc. Later in the thesis I ask what difference is made when we set $\lambda$ optimally. The second question is whether the measure of inflation used should be CPI or domestic output price inflation. And the last question is whether this form of the loss function is appropriate for the small open economy. Adolfson (2007) argues that such a loss function is suitable for an open economy with incomplete exchange rate passthrough, since the relative price distortion arising from sticky domestic and import prices can be taken into account by stabilizing CPI inflation. Smets and Wouters (2002) find that the social cost of relative price variability is minimized by a weighted average of domestic price inflation and import price inflation. Using a quadratic approximation of the average utility, Benigno (2004) shows that a weighted mean of the two regional inflation rates in an optimal currency area is the appropriate objective for monetary policy, given nominal rigidities in both regions. On the other hand, Malik (2005), Kirsanova, Leith, and Wern-Lewis (2006) all find that the loss function for an open economy will involve more terms. Because the welfare criterion (loss function) plays a crucial role in determining the optimized reaction coefficients when assessing alternative simple monetary policy rules, we need to clarify the above questions. 
The fifth issue on which this thesis focuses is whether the simple money rule depends upon the information available to the central bank. That is, in the presence of price stickiness, money is non-neutral so that monetary policy can affect efficiency. The efficiency of the monetary policy, however, is a function of its design and this, in turn, is affected by the reliability and accuracy of information used in its formation. Generally speaking, policymakers face two types of information problems: (1) uncertainty about the true structure of the economy; and (2) uncertainty about the true values of such macroeconomic variables as GDP, the GDP deflator, the Wicksellian natural interest rate, and the natural level of output, etc. One approach to answering the first is to search for monetary policy rules that work well across a wide range of structural models, see Levin et al. (1997). In the face of uncertainty about the true value of macroeconomic variables, policymakers design optimal strategies to extract information from noisy signals, see Aoki (2003), Orphanides (2003), and Svensson and Woodford (2003). It is worth emphasizing that data uncertainty issue can be mitigated by information extracting procedures but often cannot be completely resolved. Thus generally the policy maker will always face information uncertainty in deciding on its monetary policy. Given the generality of this condition, the specific question I raise is whether and how the policy rule that optimizes social welfare changes as a function of the information set available to the policy maker.

Finally, the targeted level of output often used to measure the output gap is its steady state value while the inflation target is often taken to be a simple constant (often set at zero). Similarly, the real interest rate (the Wicksellian real interest rate) used in the policy rule is often simplified to be some constant value, such as 2 percent (Taylor 1993). 
In the model of chapter 2 , however, we argue that the targeted level of real output should be given by the output level that would arise under perfectly flexible prices (in a model where subsidies/taxes correct for monopoly distortion). This also implies that in an economy exposed to both domestic and external shocks, neither the inflation rate nor the interest rate under the perfectly prices would be fixed, instead changing as the economy is hit by different shocks. The question is then, given that the central bank wishes to minimize the domestic welfare distortions that arise under sticky prices, what are the implications for policy when the central bank targets the Wicksellian natural rate and its changing inflation target.

\subsection{Methodological Underpinning}

As Walsh (2003) and others have argued, the standard Keynesian approach to monetary policy analysis prior to the early 1990 s combined the assumption of nominal price rigidity with a simple structure for linking the quantity of money to aggregate spending. This linkage often used a simple quantity theory equation in which nominal demand was set equal to nominal supply (as in traditional IS-LM analysis) with a random disturbance included. Although this approach has proved useful in addressing a wide range of monetary policy issues, its theoretical foundations were weak and unmotivated. To address this issue a new type of the model is developed and popularized by Yun (1996), Goodfriend and King (1997), Rotemberg and Woodford (1995, 1997), McCallum and Nelson (1999), Clarida, Gali, and Gertler (1999), Svensson and Woodford (1999), Woodford $(1999,2003)$, among others. The new model is now built on the optimizing 
behavior of economic agents and is often referred to as new Keynesianism, which has become the workhorse of Keynesian monetary policy analysis.

A similar innovation occurred in open economy macroeconomics. Traditionally, the analysis of monetary policy in an open economy was based on the static framework developed by Mundell (1963) and Fleming (1962) and made dynamic by Dornbusch (1976). As such it is often referred to as the Mundell-Fleming-Dornbusch model. Just like the IS-LM framework used in the closed economy setting, the Mundell-FlemingDornbusch model played an important role in the analysis of the open economy. However, it had similar drawbacks. One salient weakness was that its aggregate macroeconomic relationships were specified directly rather than derived from assumptions about the optimizing behavior of individual and firms. As a result, the model was of limited use for conducting alternative monetary policy analysis since it was unable to link aggregate outcomes back to the welfare of the agents in the model. The motivation to re-develop microfoundations to overcome this drawback led an area of economics widely described as "new open economy macroeconomics" (henceforth NOEM). The following features are normally captured by a NOEM model: economic behavior based on optimization, sticky prices and/or wages in at least some sectors of the economy, the incorporation of stochastic shocks, and the evaluation of monetary policy based on households' utility. The seminal work in this area is Obstfeld and Rogoff (1995a, 1996). In their classic book (1996) a two-country model is used in which all of these features are included. Other important contributions include Betts and Devereux (1997, 1998), Kollmann (1997, 1999, 2002), Gali and Monacelli (2002), Ghironi (1999), Svensson (2000), Benigno and Benigno (2000), Chari, Kehoe and McGrattan (2000), Smets and 
Wouters (2000), Clarida, Gali, and Gertler (2001), Corsetti and Pesenti (2001, 2005), Choudhri, Faruqee, and Hakura (2005), Sutherland (2005), among many others. ${ }^{6}$

This is the spirit in which I develop the dynamic stochastic general equilibrium open economy model that forms the basis of this thesis. The model solves the representative household and firm optimization problems facing sticky prices and stochastic shocks as the foundation of aggregate expenditure and supply relations. Alternative policy rules for the central bank in this economy are then evaluated relative to the potential welfare gains that could be realized by these representative agents.

\subsection{Basic Features and Contributions}

The starting point for my small open economy analysis is Gali and Monacelli (2002). Following them I assume that the world economy consists of two parts: a home economy and foreign one, the latter called the rest of the world. Compared to the rest of the world, the home economy is assumed to be small so that developments there have no impact on the rest of the world. A representative household in the home economy is modeled as maximizing its expected utility by purchasing both home produced and imported goods for consumption financed by its supply of specialized labor and the profit from its ownership of domestic firms. A typical firm is assumed to hire specialized labor to produce differentiated domestic goods that are used for domestic consumption and export, and to set its prices of domestic goods by using Calvo's (1983) pricing

\footnotetext{
${ }^{6}$ Some of the literature on NOEM can be found on http://www.geocities.com/brian $\mathrm{m}$ doyle/open.html, which is a New Open Economy Macroeconomics homepage, created and maintained by Brian Doyle, and on the web site, http://www. geocities.com/monetaryrules/mpoe.htm, which is about Monetary Policy Rules in Open Economies, created and maintained by Gianluca Benigno, Pierpaolo Benigno, and Fabio Ghironi. In addition, Lane (2001) and Bowman and Doyle (2003) provide good surveys on NOEM.
} 
specification. From this I obtain two key equations of motion by log-linearizing movements about the equilibrium conditions for the small open economy: the first is the new Keynesian Phillips curve and the second is the IS curve. The government's behavior is not modeled explicitly except to collect lump-sum taxes and subsidize firms (to offset monopolistic power) and to facilitate money transfers to households. The monetary authority is to set the short-term interest rate consistent with maintaining a zero expected rate of inflation.

While Gali and Monacelli (2002) deliver a model that is well specified and solvable, the implications of their model for optimal monetary policy are dependent on the assumption of a number of strong conditions that do away with important features of the real world. The following is a list of special features that distinguish my analysis from that of Gali and Monacelli, features and contribution can be assessed relative to Gali and Monacelli's results for optimal monetary policy.

(1) Incomplete exchange rate pass-through. Gali and Monacelli (2002) assume that the law of one price holds for individual goods at all times (both for import and export goods), which implies that the exchange rate pass-through is complete. This conflicts with well-established empirical facts. For example, Goldberg and Kentter (1997) find that the fraction by which changes in the exchange rate are passed-through to internal import prices is typically around 0.5 for one year. Similarly, Campa and Goldberg (2002) test the simple versions of the LCP and PCP hypotheses for complete passthrough to import prices and find that both hypotheses are rejected for most countries in their sample of OECD countries. ${ }^{7}$ To incorporate this feature into my model, I assume

\footnotetext{
${ }^{7}$ Some extensive theoretical and empirical surveys can be found in Rogoff (1996), Engel (1993, 2002), Rogers and Jenkins (1996), and Choudhri et al. (2005).
} 
that there are no transportation costs or trade barriers between the small open economy and the rest of the world so that the law of one price holds when imported goods arrived at domestic docks. However, in setting the domestic currency price for imported goods, importers face quadratic adjustment costs that generate deviations from the law of one price in the short run. In the export market, however, I assume that the law of one price continues to hold.

(2) Incomplete asset markets. As argued by Obstfeld and Rogoff (1995b, 1996), the assumption of complete asset markets is not realistic in a model with imperfections and rigidities in its goods markets because with nominal rigidities monetary policy will affect real variables including the current account. The fact that current account imbalances have increased steadily in the rich countries over the past 20 years (Blanchard, 2007), implies that asset markets are not complete in practice either. In the literature, however, many economists make this unrealistic assumption for the sake of simplicity and convenience (see Chari et al., 2000, Clarida et al., 2001, and Gali and Monacelli, 2002, for example). In doing so the interaction of current account dynamics with inflation, output, and other important macroeconomic variables is shut down. To restore this channel, I assume that the representative household has access to a one-period risk-free domestic bond and a risky foreign bond. The foreign bond is assumed to be denominated in the foreign currency and traded freely internationally subject to a risk premium which depends on both the net foreign asset position of domestic economy and a time varying shock to the risk premium. The inclusion of either of these two features (incomplete exchange rate pass-through and incomplete asset markets) leads monetary policy analysis 
for an open economy to be fundamentally different from that involved in a closed economy setting.

(3) Real balances. Real money balances are often omitted from the household's utility function as in Gali and Monacelli (2002) and now the omission of a motivation for money holdings has gradually become a fashion in the NOEM literature (see Woodford, 2003). In part this is because interest rate setting by the central bank makes the money supply endogenous and in part because that the impact of money on household utility is assumed to be very small so that its presence can be neglected. In chapter three we show that if real balances are omitted, then the interest rate will not appear in the loss function that is derived from household utility. This, in turn, implies that the central bank will be less constrained in adjusting its short-term interest rate to bring other targeted variables back into line and so more tolerant of volatility in the interest rate. This hypothesis, however, does not get much support from actual central bank behavior since central banks are observed to behave cautiously in changing interest rates. To help account for this phenomenon (in addition to motivating money's use in the economy) I incorporate real money balances into the household's utility function. As we will see, the inclusion of real balances results in one more term than usual (the interest rate) appearing in the loss function.

(4) Price indexation. Price stickiness in my model (as in many others) is incorporated by assuming the firms face Calvo's price-setting mechanism. This gives rise to a new Keynesian Phillips curve characterized by the absence of inflation inertia. To capture the inflation inertia often observed across countries I introduce price indexation into the model following Christano et al. (2001). The basic idea is that a firm will either 
receive a signal to change its price and will do so optimally, or it will not, in which case it follows a simple price indexation rule to automatically update its price. This automatic adjustment mechanism is also applied to importers.

(5) Targeted gaps. The model in Chapter 2 is written in terms of the relevant welfare measures--the gaps arising between actual and flexible price (socially optimal) values. In our setup, then, two basic types of distortions from the social optimum are built in. The first arises from monopolistic (rather than perfect) competition and the second arises from sticky prices. This gives rise to several types of efficiency gaps. For example, the gap between (the logarithm of) actual and steady state output can be decomposed into: (1) the gap between the competitive (efficient) level of steady state output and the steady state level of output, defined as $x^{*}=\ln \left(Y^{*} / \bar{Y}\right)$, where $Y^{*}$ denotes that the efficient level of output that would arise in absence of monopoly distortion and any shocks, and $\bar{Y}$ denotes the steady state level of output arising from the economy in absence of shocks and the distortion arising from prices stickiness but with the distortion from the market power owned by the firm; (2) the gap between (the logarithm of) the natural level of output and the steady state level of output, defined as $x_{t}^{f}=\ln \left(Y_{t}^{f} / \bar{Y}\right)$, where $Y_{t}^{f}$ denotes the natural level of output that would arise under complete price flexibility; (3) the gap between (the logarithm of) actual and steady state output, defined as $x_{t}^{a}=\ln \left(Y_{t} / \bar{Y}\right)$; and (4) the gap between actual and the flexible price output level, defined as $x_{t}=\ln \left(Y_{t} / \bar{Y}\right)-\ln \left(Y_{t}^{f} / \bar{Y}\right)=\ln \left(Y_{t} / Y_{t}^{f}\right)$. The relationship among these concepts is illustrated in Figure 1.1. 


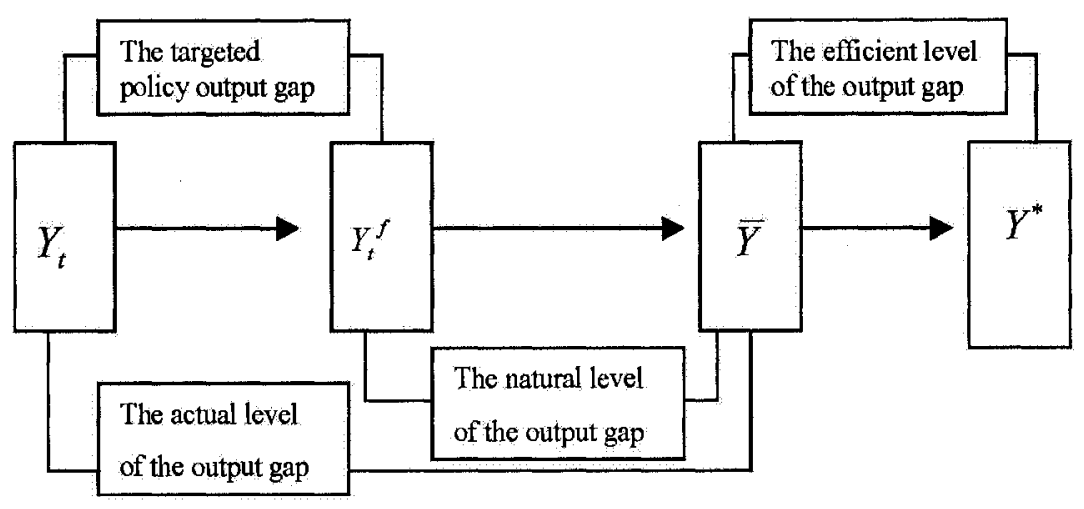

Figure 1.1 the output gaps

A natural question that arises is which gap should be targeted by the policy maker? In my analysis, the first distortion is dealt with through an optimal output subsidy, therefore, the eliminating the monopoly distortion in this way means that $x^{*}$ is zero, while the second is fixed by using monetary policy. As Woodford (2003) and others have argued, the distortion that should be corrected by policy is measured by the gap between the actual level and the level that would arise under perfectly flexible prices, that is, $x_{t}$ is the output gap that policy should target. This is in contrast to what is often used, $x_{t}{ }^{a}$, the gap between actual output and its steady state value. To emphasize the importance of this point for policy we write the variables of the model in terms of the gaps that arise between their actual level and the level that would arise under perfectly flexible prices.

(6) Two inflation measures. As discussed the above, which type of inflation should be targeted is still open. To address this issue the model is written in terms of both domestic output price inflation and CPI inflation. Policy evaluation proceeds on the same basis. 
(7) Loss function. As discussed earlier, the loss function employed to assess alternative monetary policies is often arbitrarily specified and lacks appropriate microfoundations. Hence although such a loss function seems to work, why it works seems to bewilder economists. In relation to this problem, Rotemberg and Woodford (1999) and Woodford (2003) have done pioneering research to show that a second-order Taylor series expansion of the representative household's utility function results in a loss function that has exactly the same form as that commonly used in the literature. The difference is that the value of $\lambda$ is now defined in terms of the structural parameters of the model rather than simply assigned. Following Rotemberg and Woodford (1999) I derive in Chapter 3 the loss function for the small open economy incorporating all the special model features described above.

(8) The information available to the policy maker. In many of the models developed to analyze policy, the central bank or monetary authority uses the contemporaneous values generated by the model to invoke optimal policy. However, in the real world the central bank often has insufficient information to observe or calculate the true value of the variables needed to formulate optimal policy. To reflect these circumstances, four information cases are considered. The first shows that when the central bank can access "full information", i.e., all relevant contemporaneous variables (such as GDP and the price index), can be observed, and all necessary unobservable variables (such as Wicksellian natural interest rate and the natural level of output) can be calculated. This serves as our benchmark full information case. Next we assume that the central bank can observe the current value of all observable variables, but cannot get enough information to compute accurately the unobservable variables and hence must use 
steady state values instead. The third case assumes that the central bank knows all variables, but only with a period lag. The last case assumes that all observable variables can be known accurately but also with a lag, and all unobservable variables cannot be computed accurately but must use their steady state values instead. The different information cases are used to analyze if and how optimal simple monetary policy changes with the withdrawal of accurate information.

Note that as in Aoki (2003) and Svensson and Woodford (2004), the information structure I adopt involves an important asymmetry. ${ }^{8}$ Specifically, private agents as a group (individuals and firms) possess more information than does the policy maker. For convenience, I have abstracted any information uncertainty facing private agents by assuming that they have complete knowledge about the state of the economy affecting them individually. It follows that the central bank cannot possess more information about the state of the economy than does the collectivity of private agents, but why should it possess less? One set of reasons for why current information is not fully utilized by the central bank emphasizes the cost of collecting and evaluating timely individual information. In general, individual production and consumption decisions made by firms and households are not as dependent on aggregate data as is the policy decision of the central bank. Each firm, for example, needs to know only its private production cost and the prices of close substitutes for the good it produces, but it does not typically need to know all industry prices. Moreover, the individual private agent has no private incentive to transmit the information it posses to the central bank (nor could the central bank

\footnotetext{
${ }^{8}$ Aoki (2003) and Svensson and Woodford (2004) use measurement error to distinguish between private and public decision makers. My structure emphasizes the absence rather than the uncertainty of information. The case of symmetric uncertain information is considered by Svensson and Woodford (2003). There both the central bank and the private agents have access to the same partial information.
} 
process the receipt of all that individual information costlessly). Hence while each individual firm has more information about the supply shock impacting it than does the central bank, the firm is motivated to act only in relation to its private benefit and cost rather than the social equivalent and in acting on this basis produces market signals that compact the different forms of relevant information available to each agent. The cost of interpreting the information transmitted by private individuals through markets may then result in the central bank having more aggregate information than any single agent, but having less accurate information than private agents have collectively in their possession. It is the costs of accessing all the relevant information held by individual private agents that results in the central bank having less accurate information.

(9) Observational mistakes in interpreting the information available to individuals and firms. In Chapters 2, 4, 5 and 6, we examine how the central bank adapts to ever greater degrees of data deficiency, while implicitly private agents access information perfectly. In Chapter 7 we reverse this information structure. First private agents are assumed to observe current aggregate domestic and import good price levels with measurement error, whereas the monetary authority is assumed to measure these variables without error. The analysis then considers how the money rule responds when the measurement issue is extended to the central bank.

It will be noted that some of features listed above (but not all) have been modeled in more restricted contexts by other authors. For example, Monacelli (2003) incorporates incomplete exchange rate pass-through into his analysis, while Malik (2005) considers the impact of incomplete asset markets on simple policy rule performance. In the most recent literature, Dib (2003), Ambler, Dib, and Rebei (2004), Adolfson (2007) all present 
more simplified models that incorporate both incomplete exchange rate pass-though and incomplete asset markets. Moreover, Dib (2003) and Ambler et al. (2004) question only the policy performance of a modified Taylor rule in terms of an estimated model of a small open economy, while Adolfson (2007) focuses on the impact of different degrees of exchange rate pass-through on a subset of simple optimal rules. In none of these cases are the authors interested in how the performance of different simple money rules respond to changes in the information set held by the central bank. This relative performance of money rules under different information settings is the central concern of my analysis. In the chapters below I will show that when the information availability issue is ignored, what is for us Case 1, the marginal welfare gain from incorporating external considerations such as the exchange rate is quite small. However, when as the information on which the central bank becomes less current, as in Cases 2, 3, and 4, the marginal welfare gain of including these factors is increased significantly.

It is important to also emphasize that a concern with information availability is only one dimension that differentiates my analysis from others in the same tradition, such as Dib (2003) and Adolfson (2007). Among the most important features that separate my thesis from others are: first, writing the model, the loss function and the policy rules all in terms of the appropriate "policy gap" rather than "gap between actual and steady state level"; 9 second, evaluating the alternative policy rules using a welfare criteria derived from the representative household's utility function, ${ }^{10}$ and third, none of the other authors motivate money use in their analysis. In relation to the last point, money's use in our model is motivated through the incorporation of real balances in the household's utility

\footnotetext{
${ }^{9}$ Malik (2005) does write its model and the policy rule in terms of "policy gap".

${ }^{10}$ Dib (2003), Malik (2005), and Adolfson (2007) all use ad hoc loss functions.
} 
function. ${ }^{11}$ Because of this the loss function has one more term, that is, the variance of interest rate. This provides a theoretical reason for why interest-rate smoothing should appear in the money rule (see Chapter 6).

\subsection{Structure of the Thesis}

The thesis is organized in the following manner. In Chapter 2, I develop and solve a forward-looking general equilibrium model of a small open economy. The model is characterized by the price rigidities and indexation, incomplete exchange rate passthrough, incomplete asset markets, and four types of shocks. The model is then loglinearized about its steady state and structured into the form of a dynamic stochastic general equilibrium model.

Two points need particular emphasis. The first is related to what can be achieved by monetary policy. In many early models, particularly those that focused exclusively on demand side shocks, the use of an interest rate to regulate aggregate demand could simultaneously reduce both the inflation and output gaps. In this sense there was no need to tradeoff inflation and output in the choice of an optimal policy. Setting the appropriate Wicksellian interest rate could reduce welfare losses to zero. However, in models that also incorporated supply side shocks, such as in papers by Clarida et al $(1999,2001)$, the presence of a cost-push shock required the policy maker to choose between inflation and output variability and so face a policy tradeoff in minimizing welfare loss. In such cases the weights placed on inflation and output in the loss function matter. In my analysis framework, while no cost-push inflation is built explicitly into the analysis, the model

\footnotetext{
${ }^{11}$ This feature is modeled in Dib (2003) in a bit different way. Actually, the form of the household's utility function is different from mine.
} 
does contain productivity shocks to the supply side. Moreover, the economy is subject to two types of external shocks in the presence of both incomplete price pass through and incomplete asset markets. As the impulse response analysis in Chapter 4 will show, the presence of these factors prevents the central bank from being able to eliminate all welfare loss even in Case 1. Hence the presence of multiple distortions means that the central bank cannot hit its all targets simultaneously by adjusting the short-term interest rate. This is further compounded by the lack of relevant information. The result is that the Wicksellian natural rate may not be able to capture all necessary information required to eliminate all gaps so that the weighting of policy goals becomes an important part of the optimal solution.

The second point is the linear-quadratic nature of the optimization problem. Typically in the economic literature, an optimal control problem is solved in a linearquadratic framework, that is, with a linear model and a quadratic welfare loss function (Chow, 1975). Following in this convention, the model I set up in Chapter 2 is written in this form. ${ }^{12}$ However, it has also been shown that for welfare evaluation with DSGE models, first-order approximations about the equilibrium condition may be inadequate. For example, Kim and Kim (2003) provide a simple example of a model in which welfare appears higher under autarky than under complete markets, because the first-order approximation does not account for the effects of the second order terms on the welfare. ${ }^{13}$ Woodford (2001), however, describes several conditions under which first-order

\footnotetext{
${ }^{12}$ Another motivation for writing the model linearly is that I want to derive explicitly those variables that arise under perfectly flexible prices, e.g., the Wicksellian natural interest rate and natural level of output. ${ }^{13}$ For a more general discussion, see Kim et al. (2003).
} 
approximations in DSGE models are adequate for evaluating monetary policy. These conditions hold in my model so that the conclusions obtained below should be reliable. ${ }^{14}$

Chapter 3 is devoted to the derivation of the appropriate welfare loss function for this small open economy. As mentioned above, the loss function is derived from the household utility function in the spirit of Rotemberg and Woodford (1999). Two versions of that loss function are presented. The first is a linear combination of four variables: inflation, the output gap, the real exchange rate, and the law of one price gap. This is derived by following the established practice of suppressing the presence of real money balances in the household's utility function (see Woodford, 2003). When real balances are accounted for in the household's utility function, a second loss function can be derived. Compared with the first, there is one additional term in the loss function that wasn't there before--the short-term interest rate. Note that the real exchange rate can be replaced in the loss function by the terms of trade, but cannot be substituted out of the loss function by combining it with other existing terms to narrow down the loss function. Similarly, the law of one price gap cannot be rewritten in a form that allows it to disappear from the loss function.

Chapters 2 and 3 provide the foundation for monetary policy analysis. Specific monetary policy analysis starts in Chapter 4 . Because the model is analytically complex, I follow current convention and calibrate the structural parameters of the model to conduct a quantitative simulation of the response of the economy to its real shocks and

\footnotetext{
${ }^{14}$ The conditions proposed by Woodford are as follows. (1) The distortion due to market power is sufficiently small. (2) The average rate of inflation is low. (3) The form we choose to express our approximate loss function should be employed properly. These three conditions are held in our analysis. First of all an output subsidy is imposed to fix the distortion arising from the firm's market power. Therefore the distortion due to market power is zero in principle. Second, the target inflation rate is assumed to be zero. Finally, following Rotemberg and Woodford (1999), Woodford (2003), I use the appropriately form to express the approximate loss function.
} 
monetary policy alternatives. The focus of Chapter 4 is given the reaction coefficients typically used in the literature, what simple monetary policy rules work best in minimizing welfare losses. This question is considered for four different information settings. For each information set available to the central bank, I compare the outcome from using five different simple policy rules with and without interest-rate smoothing. Then to shed light on the difference that would be made by targeting inflation measured in domestic output rather than in consumer prices, I compare each rule for the two versions of the model. In addition, I compare the use of a constant inflation target with a period by period varying inflation target. Finally, I also compare inflation forecast to nonforecast rules.

Perhaps the most interesting finding of Chapter 4 is that across the different money rules and information cases the Taylor rule works the best. This is consistent with findings of Clarida et al (2001) and others. However, since the reaction coefficients in the policy rule are ad hoc (simply picked from the literature), a natural question raised is whether the same result will hold when the reaction coefficients are solved for optimally. This leads to Chapters 5 and 6 . Chapter 5 answers the question of what is the fully optimal monetary policy rule given the model and loss function laid out in Chapters 2 and 3. The fully optimal policy rule is solved under commitment. It is solved using the approach proposed by Blanchard and Kahn (1980) and extended by Klein (2000), Sims (2001), and others.

Given that the cost of implementing the fully optimal rule is prohibitive, I turn in Chapter 6, to reinvestigate choice among simple policy rules but by using the optimized value of the reaction coefficients in the money policy rule. It is important to note here 
that all simple rules are sub-optimal in the sense that some coefficient values are arbitrarily set equal to zero. In chapter 6 , then, I redo most of the tests undertaken in Chapter 4 while solving simultaneously for the optimal reaction coefficients to be used by the central bank. The analysis begins by examining optimal simple policy rules with partial interest-rate smoothing with the coefficient on the lagged interest rate set at 0.8 . This starting point is chosen because of its consistency with the empirical evidence ${ }^{15}$ and because of its widespread use in the current literature (see Taylor, 1999, Adolfson, 2007, for example) makes for easier comparison of my results with others. All other reaction coefficients are solved optimally. From this beginning point $I$ then relax this assumption and let the coefficient on the lagged interest rate be determined optimally. After examining this set of optimal simple rules under interest-rate smoothing, I examine the optimal simple policy rules without interest-rate smoothing. Finally, to assess whether some of the tentative hypotheses advanced to explain these results have validity or reliability, various robustness tests and experiments are conducted.

In the previous chapters it was assumed that only the central bank faced a significant data deficiency when making its optimal policy decision. Private agents--both individuals and firms--were assumed to have perfect information in making their optimal choices. In the last chapter, we reverse this scenario by assuming that central bank has access to perfect information, while private agents can observe the current period domestic and import price levels only with error. The question of interest is whether this change in the asymmetry of information affects the properties of the optimal simple policy rules for a small open economy. This requires the re-derivation of the model to incorporate measurement error, but employs the same loss function as used in previous

\footnotetext{
${ }^{15}$ It is supported by theoretical studies as well, see Aoki 2003.
} 
chapters to allow for the evaluation of the relative performance of the same five alternative optimal simple policy rules. This measurement issue is then extended to the monetary authority.

\subsection{Conclusions}

At present the Taylor rule is the monetary policy rule most often advocated in the literature, even for the case of a small open economy subject to external shocks. It was also the money rule that performed best in our small open economy model when the reaction coefficients assigned were the values most often used in the literature. However, the conjecture that the Taylor rule would continue to perform best when the reaction coefficients are found optimally has proved misleading if not entirely wrong. In our model, it is always optimal to augment the Taylor rule with some factor that allows for the incorporation of relevant external complications - in our case either the exchange rate gap or the law of one price gap, although in some situations the marginal welfare gain from incorporating the exchange rate in some form is small. This finding is robust across our four cases of different information availability. As discussed earlier, Ball (1999), Monacelli (2003), and Malik (2005) have all derived similar conclusion but based on narrower theoretical foundation. For example, Ball's conclusion is obtained within a backward-looking model, while Monacelli's monetary policy arises only in the context of a low exchange rate pass-through environment. Finally, Malik's monetary policy outcome is not optimal since the reaction coefficients in the policy rule are based on the literature rather than found optimally. 
The choice of the index in which to measure the inflation rate target has been another interesting policy question. While it appears that most inflation-targeting central banks have chosen to measure the rate of inflation in terms of some version of the CPI, our analysis finds that despite the appearance of the consumer price index as an important variable within the model, a central bank that bases its policy rule on domestic output prices will normally perform better overall than if it focuses on a consumer price index. This policy prescription is not unique to this model but has been also found by Clarida et al (2001). ${ }^{16}$ As such our analysis reinforces this policy prescription.

Third, the addition of the lagged interest rate to an optimal simple policy rule typically enhances the level of social welfare and hence policy performance. The reason why this is the case was explored at length and appears to be for a combination of reasons - an ability to capture information missed in other policy instruments, the fact that welfare depends directly in interest rate variability, and as a way of establishing and maintaining commitment. Perhaps most controversially, we find in section 6.3 that the optimized value of the coefficient on the lagged interest rate is greater than one. This is consistent with the findings of authors such as Rotemberg and Woodford (1999), but completely different from the empirical estimates usually used in the literature (most often ranging between 0.7 and 0.9 ). To the extent that my model captures some of the more important features of small open economies, it would imply that the central banks of these economies have been too timid in practice.

Fourth, our analysis suggests that the marginal welfare gain from incorporating foreign exchange considerations and/or lagged interest rates falls as the relevant

\footnotetext{
${ }^{16}$ Clarida et al. find that the central bank should target domestic inflation and allow the exchange rate to float when there is perfect exchange rate pass-through.
} 
information available to the policy maker improves. This is perhaps our most relevant policy finding. It suggests that as a practical proposition, the less well developed is the statistical information available to a small open economy, the larger will be the gain from including some measure of the size of the external shock facing the economy and/or lagged interest rates in the monetary policy rule. Such a finding could not be observed in studies in which information availability is not considered as a relevant policy problem (as in Monacelli 2003, Adolfson 2007).

Fifth, the introduction of measurement error increases the degree of incompleteness in the information available to the monetary authority and hence increases welfare loss. Nevertheless, regardless of whether that information loss arises in relation to private agents or policymakers, it does not alter the relative efficiency of the alternative policy rules. In our analysis of the impact of measurement error, the comprehensive policy rule is always at the top of the list of optimal money rules, while the inflation targeting rule always appears at the bottom.

Finally, in terms of the coefficients within the policy rule, the optimal weight that is placed on indicators that can be observed only with noise receives less weight than otherwise. An increase in an indicator's noise strictly reduces its policy weight. However, in a dynamic forward-looking general equilibrium model all endogenous variables interact. Therefore as long as one variable contains noise, the noise in that variable will be transmitted to others via individual expectations and market interaction within general equilibrium. Hence the optimal weight assigned to these contaminated indicators will also tend to fall. Because the degree of interaction depends upon the specific model, the relative weighting can decrease or increase depending upon specific circumstance. 


\section{Chapter 2}

\section{A Small Open Economy Model with Incomplete Exchange Rate Pass-through}

Suppose that the world economy consists of two parts: a home economy and a foreign one, the latter called the rest of the world. Compared to the rest of the world, the home economy is assumed to be small so that developments there have little impact on the rest of the world. This implies that policy makers deciding on domestic monetary policy can take foreign variables, such as foreign output, prices, inflation rates, and interest rates as given or as exogenous stochastic variables.

The model we use to analyze monetary policy in this context is a variant of the dynamic New Keynesian model applied to a small open economy such as Canada (see Gali and Monacelli, 2002). The model consists of a representative household who purchases both home produced and imported goods for consumption from its supply of specialized labor and the profits from its ownership share of domestic firms. Households are also assumed to be able to reallocate consumption over time by accessing a oneperiod risk-free (non-contingent) domestic bond and a risky foreign bond. The foreign bond is assumed to be denominated in the foreign currency and traded freely internationally subject to a risk premium which depends on both the net foreign asset position of domestic economy and a time-varying shock to the risk premium (Adolfson, 2005). 
The model assumes a large number of identical firms that hire specialized labor to produce differentiated domestic goods that are used for both domestic consumption and export. For simplicity we assume that there is no capital accumulation and there are no intermediate goods. The labor market is assumed to be competitive with flexible wages but each domestic producer is assumed to be a monopolistic competitor setting prices by using Calvo's (1983) pricing specification. We assume that there are no transportation costs or trade barriers between the small open economy and the rest of the world so that the law of one price holds when imported goods cross the domestic border. However, in setting the domestic currency price for these differentiated goods, importers face quadratic adjustment costs that will generate deviations from the law of one price in the short run. In the literature this is described as incomplete exchange rate pass-through. For simplicity we assume that the law of one price holds for domestic exporters.

In our setup, the monetary authority is assumed to maximize community welfare by choosing as its policy instrument the short-term interest rate. The policy instrument is used to correct distortions arising in the model from stochastic real shocks impacting on the model in the presence of sticky prices. The secondary distortion that results from the presence of monopolistic competition in the model is dealt with separately through an optimal output subsidy. Aside from these tax/transfer activities, government behavior is not modeled explicitly.

\subsection{The Representative Household}

The small open economy consists of a continuum of households indexed by $i \in[0,1]$. The representative household seeks to maximize the expected value of the 
discounted sum of time separable utilities subject to an intertemporal budget constraint. Suppose then that total expected utility can be denoted as

$$
U_{0}=E_{0} \sum_{t=0}^{\infty} \beta^{t} u\left(C_{t} \frac{M_{t}}{P_{t}}, N_{t}, \chi_{1 t}\right)
$$

where $\beta \in(0,1)$ is the subjective discount factor, $C_{t}$, denotes a composite bundle of consumption goods, $M_{t} / P_{t}$ is the level of real money balances held by the household, and $N_{t}$ is the proportion of household time devoted to the labor market (so that $1-N_{t}$ is the proportion of time enjoyed as leisure by the representative household). Following Woodford (2003), we assume that each of the differentiated goods is produced with a specialized type of labor and that the representative household supplies each type of specialized labor. In this case, $N_{t}=\int_{0}^{1} N_{t}(i) d i$, where $N_{t}(i)$ is the quantity of labor of type i supplied by the household. ${ }^{17}$

While the household derives utility from consumption, holding real money balances, and taking leisure, the presence of an exogenous disturbance $\chi_{1 t}$ in the utility function implies that household utility is subject to a stochastic taste shock. This is given more specific form through the per-period utility function

$$
u\left(C_{t} \frac{M_{t}}{P_{t}}, N_{t}, \chi_{1 t}\right)=\frac{\chi_{1 t}}{1-\sigma} C_{t}^{1-\sigma}+\frac{1}{1-\varsigma}\left(\frac{M_{t}}{P_{t}}\right)^{1-\varsigma}-\frac{1}{1+\mu} \int_{0}^{1}\left(N_{t}(i)\right)^{1+\mu} d i
$$

Here $1 / \sigma$ denotes the intertemporal elasticity of substitution in consumption, $1 / \varsigma$ is the interest elasticity of money demand. $\mu$ is the elasticity of marginal disutility with

\footnotetext{
${ }^{17}$ This implies that the import sector uses no labor, merely re-pricing goods imported from abroad.
} 
respect to labor supply ${ }^{18}$. The shock to the utility function makes the consumption weight in the utility function stochastic. Hence a change in $\chi_{1 t}$ represents a preference shock to consumption, where $\chi_{1 t}$ is assumed to follow the first-order autoregressive process given by

$$
\ln \chi_{1 t}=\rho_{1} \ln \chi_{1, t-1}+\varepsilon_{1 t},
$$

and where $0<\rho_{1}<1$ and the error term, $\varepsilon_{1 t}$, is normally distributed with zero mean and standard deviation $\sigma_{1}$.

Composite consumption, $C_{t}$, is assumed to consist of a bundle of domestic and foreign produced goods, that is,

$$
C_{t}=\left[(1-\alpha)^{\frac{1}{\eta}} C_{H, t}^{\frac{\eta-1}{\eta}}+\alpha^{\frac{1}{\eta}} C_{F, t}^{\frac{\eta-1}{\eta}}\right]^{\frac{\eta}{\eta-1}},
$$

where $C_{H, t}$ and $C_{F, t}$ are, respectively, consumption bundles of domestic and imported foreign goods. $\eta>0$ is the elasticity of substitution between domestic and foreign goods and $\alpha$ denotes the steady-state share of import goods in household total consumption, Following Dixit and Stiglitz (1977), we assume that $C_{H, t}$ and $C_{F, t}$ are constant elasticity of substitution aggregators defined, respectively, as

$$
\begin{gathered}
C_{H, t}=\left[\int_{0}^{1} \mathrm{C}_{\mathrm{H}, t}(i)^{\frac{\theta-1}{\theta}} d i\right]^{\frac{\theta}{\theta-1}}, \\
\text { and } C_{F, t}=\left[\int_{0}^{1} \mathrm{C}_{\mathrm{F}, \mathrm{t}}(z)^{\frac{\theta-1}{\theta}} d z\right]^{\frac{\theta}{\theta-1}},
\end{gathered}
$$

\footnotetext{
${ }^{18}$ Note that $1 / \mu$ denotes the elasticity of labor supply with respect to real wage.
} 
where $C_{H, t}(i)$ represents the household's consumption of the domestically produced good $\mathrm{i}$, and $C_{F, t}(z)$ represents the household's consumption of imported good $z$. We use $\theta$ to denote the elasticity of substitution within each category of composite goods, $C_{H, t}$ and $C_{F, t}$, and assume that $\theta>1$.

The problem faced by the representative household is to choose the values for $C_{t}, M_{t} / P_{t}$, and $N_{t}(i)$ such that lifetime utility in (2.1) is maximized. To that end the household can be viewed first as minimizing the cost of purchasing $C_{H, t}(i)$ regardless of the level of $C_{H, t}$ that is decided upon. More formally this household choice problem is to

$$
\operatorname{Min} \int_{0}^{1} P_{H, t}(i) C_{H, t}(i) d i
$$

subject to

$$
\left[\int_{0}^{1} \mathrm{C}_{\mathrm{H}, \mathrm{t}}(i)^{\frac{\theta-1}{\theta}} d i\right]^{\frac{\theta}{\theta-1}} \geq C_{H, t}
$$

where $P_{H, t}(i)$ is the set of prices applicable to the domestic goods. Letting $\lambda_{1 t}$ be the Lagrangian multiplier on the constraint, the Lagrangian function can be written as

$$
L=\int_{0}^{1} P_{H, t}(i) C_{H, t}(i) d i+\lambda_{1 t}\left\{C_{H, t}-\left[\int_{0}^{1} \mathrm{C}_{\mathrm{H}, t}(i)^{\frac{\theta-1}{\theta}} d i\right]^{\frac{\theta}{\theta-1}}\right\} .
$$

The first order condition for good i, $C_{H, t}(i)$, is

$$
P_{H, t}(i)-\lambda_{1 t}\left[\int_{0}^{1} C_{H, t}(i)^{\frac{\theta-1}{\theta}} d i\right]^{\frac{1}{\theta-1}} C_{H, t}(i)^{\frac{-1}{\theta}}=0 .
$$

Plugging (2.5) into (2.7), and rearranging, we obtain 


$$
C_{H, t}(i)=\left(\frac{P_{H, t}(i)}{\lambda_{1 t}}\right)^{-\theta} C_{H, t}
$$

Substituting (2.8) back into (2.5) and solving for $\lambda_{1 t}$, we get

$$
\lambda_{1 t}=\left[\int_{0}^{1} P_{H, t}(i)^{1-\theta} d i\right]^{\frac{1}{1-\theta}}=P_{H, t} .
$$

That is, the Lagrangian multiplier, $\lambda_{1 t}$, is equal to the domestic goods price index, $P_{H, t}$. Plugging (2.9) into (2.8), the demand for domestic good i can now be rewritten as

$$
C_{H, t}(i)=\left(\frac{P_{H, t}(i)}{P_{H, t}}\right)^{-\theta} C_{H, t} .
$$

Similarly, the demand for the imported good z, $C_{F, t}(z)$, can be shown to be

$$
C_{F, t}(z)=\left(\frac{P_{F, t}(z)}{P_{F, t}}\right)^{-\theta} C_{F, t},
$$

where $P_{F, t}(z)$ is the price of import good z, and the import price index, $P_{F, t}$, is defined as

$$
P_{F, t}=\left[\int_{0}^{1} P_{F, t}(z)^{1-\theta} d z\right]^{\frac{1}{1-\theta}}
$$

Analogously, we can derive the demand for the bundle of domestic produced goods, $C_{H, t}$, and import goods, $C_{F, t}$, by minimizing their purchase cost $\left(P_{H, t} C_{H, t}+P_{F, t} C_{F, t}\right)$ subject to equation (2.4). Specifically, the representative household problem is to

$$
\operatorname{Min}\left(P_{H, t} C_{H, t}+P_{F, t} C_{F, t}\right)
$$

subject to 


$$
\left[(1-\alpha)^{\frac{1}{\eta}} C_{H, t}^{\frac{\eta-1}{\eta}}+\alpha^{\frac{1}{\eta}} C_{F, t}^{\frac{\eta-1}{\eta}}\right]^{\frac{\eta}{\eta-1}} \geq C_{t}
$$

The first order condition for $C_{H, t}$ in this internal minimum is

$$
P_{H, t}=\lambda_{2 t}\left[(1-\alpha)^{\frac{1}{\eta}} C_{H, t}^{\frac{\eta-1}{\eta}}+\alpha^{\frac{1}{\eta}} C_{F, t}^{\frac{\eta-1}{\eta}}\right]^{\frac{\eta}{\eta-1}-1}(1-\alpha)^{\frac{1}{\eta}} C_{H, t}^{-\frac{1}{\eta}}
$$

where $\lambda_{2 t}$ is the Lagrangian multiplier on the constraint in (2.13). The first order condition for $C_{F, t}$ can be written similarly as

$$
P_{F, t}=\lambda_{2 t}\left[(1-\alpha)^{\frac{1}{\eta}} C_{H_{, t}}^{\frac{\eta-1}{\eta}}+\alpha^{\frac{1}{\eta}} C_{F, t}^{\frac{\eta-1}{\eta}}\right]^{\frac{\eta}{\eta-1}-1}(\alpha)^{\frac{1}{\eta}} C_{F, t}^{-\frac{1}{\eta}}
$$

The two first order conditions (2.14) and (2.15) can be further simplified to become

$$
\begin{gathered}
C_{H, t}=(1-\alpha)\left(\frac{P_{H, t}}{\lambda_{2 t}}\right)^{-\eta} C_{t}, \\
C_{F, t}=\alpha\left(\frac{P_{F, t}}{\lambda_{2 t}}\right)^{-\eta} C_{t} .
\end{gathered}
$$

Finally, substituting (2.16) and (2.17) back into (2.4), we find

$$
\lambda_{2 t}=\left[(1-\alpha) P_{H ; t}^{1-\eta}+\alpha P_{F, t}^{1-\eta}\right]^{1-\eta}
$$

It implies that $\lambda_{2 t}=P_{t}$ so that $P_{t}$ to replace $\lambda_{2 t}$ in $(2.16)$ and (2.17), we obtain the following demand functions,

$$
\begin{aligned}
C_{H, t} & =(1-\alpha)\left(\frac{P_{H, t}}{P_{t}}\right)^{-\eta} C_{t}, \\
C_{F, t} & =\alpha\left(\frac{P_{F, t}}{P_{t}}\right)^{-\eta} C_{t},
\end{aligned}
$$


where $P_{t}$ is the consumer price index defined as

$$
P_{t}=\left[(1-\alpha) P_{H, t}^{1-\eta}+\alpha P_{F, t}^{1-\eta}\right]^{\frac{1}{1-\eta}}
$$

Turning next to the household's budget constraint, the representative household is viewed as entering period $t$ with a set of financial assets. These include its claim on the profits arising in domestic firms (including imports), its initial nominal money holdings, and a portfolio consisting of its initial holdings of a risk free domestic bond and a foreign bond denominated in units of the foreign country's currency. We assume that only the foreign bond can be traded internationally. Because the household cannot insure himself from all risks financial asset markets are incomplete and the domestic household is assumed to have to pay a premium on its foreign borrowing that depends directly on the size of its net foreign asset position, $N F A_{t}$. It then follows that because the household can accumulate or decumulate foreign assets in any period, there can arise differences between the income earned and level of consumption expenditures in period $t$. Hence in real terms, the representative household's budget constraint can be written as,

$$
\begin{aligned}
& C_{t}+\frac{M_{t}}{P_{t}}+\frac{B_{t}}{P_{t} R_{t}}+\frac{\varepsilon_{t} B_{t}^{*}}{P_{t} R_{t}^{*} \exp \left(-b N F A_{t}+\ln \chi_{2 t}\right)}+\frac{T_{t}}{P_{t}}= \\
& \frac{1}{P_{t}} \int_{0}^{1} N_{t}(i) W_{t}(i) d i+\frac{1}{P_{t}} \int_{0}^{1} D_{t}(i) d i+\frac{1}{P_{t}} \int_{0}^{1} D_{t}(z) d z+\frac{M_{t-1}}{P_{t}}+\frac{B_{t-1}}{P_{t}}+\frac{\varepsilon_{t} B_{t-1}}{P_{t}}
\end{aligned},
$$

where $M_{t}$ is the level of money holdings chosen for the end of period t. $B_{t}$ and $B_{t}^{*}$ are the country specific values in period $t+1$ dollars of one period domestic and foreign bonds chosen to be held at the end of period t. $R_{t}$ and $R_{t}^{*}$ denote, respectively, the gross nominal domestic and the risk free foreign (world) interest rate arising between $t$ and 
$t+1$. Following Benigno (2001) and Adolfson (2007), we define $\exp \left(-b N F A_{t}+\ln \chi_{2 t}\right)$

as the risk premium that the representative household must pay to borrow on world markets, a premium that increases in the size of the net foreign debt held by the representative household, and $b$ is a constant that governs the size of the risk premium. ${ }^{19}$ Because that premium is subject to a period specific shock, $\chi_{2 t}$, the time dependent risk premium will produce temporary departures from uncovered interest parity in the short run. Here $N F A_{t}=\varepsilon_{t} B_{t}{ }^{*} / P_{t}$ is the domestic value of net foreign assets held by the household, $\varepsilon_{t}$ is the nominal exchange rate, defined as the price of foreign currency (in terms of domestic currency), and $\chi_{2 t}$ is the shock to the risk premium. Analogous to $\chi_{1 t}, \chi_{2 t}$ is defined as

$$
\ln \chi_{2 t}=\rho_{2} \ln \chi_{2, t-1}+\varepsilon_{2 t},
$$

where $\rho_{2}<0$ and $\varepsilon_{2 t}$ is white noise.

In (2.21) the representative household is modeled as receiving a nominal wage rate for each unit of type $i$ labor provided, $W_{t}(i)$. The household is also assumed to own a representative share of all firms producing domestic goods so that the nominal value of dividend income (profits) received from firm $i$ is equal to $D_{t}(i)$ in period t. By assumption the representative household is also assumed to own a representative share of the profits (losses) made on each differentiated import good $z$, profits of $\int_{0}^{1} D_{t}(z) d z / P_{t}$ in period t. Finally, the household is subject to a lump-sum tax levied by the government

\footnotetext{
${ }^{19}$ Having the risk premium depend on the level of net foreign borrowing allows the model to equate differences that may arise between the household's subjective rate of time preference and the risk free world interest rate.
} 
$T_{t}$ at the start of period $\mathrm{t}$ just sufficient to cover the output subsidies made by the government (to induce efficient levels of domestic and import output).

Finally, to simplify the analysis, we assume that there is no other government spending on final goods so that any seigniorage on money creation will be rebated to households in the form of a lump-sum tax, $\mathrm{T}$, and all output subsidies paid to offset firm markups are funded through lump-sum taxes. Hence in our economy, the government budget constraint is,

$$
\tau P_{t} Y_{t}+\tau_{M} P_{F, t} C_{F, t}=M_{t}-M_{t-1}+T_{t}
$$

where $\tau$ and $\tau_{M}$ are subsidy rates motivated in greater detail later. ${ }^{20}$ Thus $\tau P_{t} Y_{t}$ denotes the subsidy paid by the government to domestic producers in period $\mathrm{t} ; \tau_{M} P_{F, t} C_{F, t}$ denotes the subsidy paid by the government to importers; and $M_{t}-M_{t-1}$ represents the seigniorage revenue arising from money creation. Note that seigniorage need not be positive.

The representative household then chooses $C_{t}, N_{t}(i), M_{t}, B_{t}$ and $B_{t}^{*}$ to maximize lifetime utility given in (2.1) subject to the flow budget constraints given in (2.21). Dynamic programming is used to solve the household's problem where the value function underlying the household's decision problem is defined as

$$
V\left(\omega_{t}\right)=\operatorname{Max}\left\{\left[\frac{\chi_{1 t}}{1-\sigma} C_{t}^{1-\sigma}+\frac{1}{1-\zeta}\left(\frac{M_{t}}{P_{t}}\right)^{1-\zeta}-\frac{1}{1+\mu} \int_{0}^{1} N_{t}(i)^{1+\mu} d i\right]+\beta E_{t} V\left(\omega_{t+1}\right)\right\}
$$

\footnotetext{
${ }^{20}$ In this model the two potentially different subsidy rates will end up being equal.
} 
Notice that the income derived from labor and dividend payments will depend upon the household's current period choice of labor supply and hence is separated from household's initial wealth $\omega_{t}$. The state variable for period $t, \omega_{t}$, is then defined as

$$
\omega_{t}=\frac{M_{t-1}}{P_{t}}+\frac{B_{t-1}}{P_{t}}+\frac{\varepsilon_{t} B_{t-1}^{*}}{P_{t}} .
$$

Updating $\omega_{t}$ one period, we obtain

$$
\omega_{t+1}=\frac{M_{t}}{P_{t+1}}+\frac{B_{t}}{P_{t+1}}+\frac{\varepsilon_{t+1} B_{t}^{*}}{P_{t+1}}
$$

Let $\lambda_{3 t}$ be the Lagrangian multiplier associated with the flow budget constraint. Thus the maximization in (2.24) is subject to the budget constraint in (2.21). The first order conditions for an internal optimum for $C_{t}, N_{t}(i), M_{t}, B_{t}, B_{t}^{*}$ and $\omega_{t}$ are then

$$
\begin{gathered}
\chi_{1 t} C_{t}^{-\sigma}=\lambda_{3 t}, \\
N_{t}(i)^{\mu}=\lambda_{3 t}\left(\frac{W_{t}(i)}{P_{t}}\right) \\
\frac{1}{P_{t}}\left(\frac{M_{t}}{P_{t}}\right)^{-\varsigma}+\frac{\beta}{P_{t+1}} E_{t} V_{\omega_{t+1}}\left(\omega_{t+1}\right)-\frac{\lambda_{3 t}}{P_{t}}=0, \\
\frac{\beta}{P_{t+1}} E_{t} V_{\omega_{t+1}}\left(\omega_{t+1}\right)-\frac{\lambda_{3 t}}{P_{t} R_{t}}=0 \\
\frac{\beta \varepsilon_{t+1}}{P_{t+1}} E_{t} V_{\omega_{t+1}}\left(\omega_{t+1}\right)-\frac{\lambda_{3 t} \varepsilon_{t}}{P_{t} R_{t}^{*} \exp \left(-b N F A_{t}+\ln \chi_{2 t}\right)}=0, \\
V_{\omega_{t}}\left(\omega_{t}\right)=\lambda_{3 t} .
\end{gathered}
$$

Updating the first order conditions for $C_{t}$ and $\omega_{t}$ one period and using all the first order conditions above, we obtain the following expressions, 


$$
\begin{gathered}
C_{t}^{-\sigma}=\beta R_{t} E_{t}\left(\frac{P_{t}}{P_{t+1}}\right)\left(\frac{\chi_{1 t+1}}{\chi_{1 t}}\right) C_{t+1}^{-\sigma} \\
C_{t}^{-\sigma}=\beta R_{t}^{*} \exp \left(-b N F A_{t}+\ln \chi_{2 t}\right) E_{t}\left(\frac{\varepsilon_{t+1}}{\varepsilon_{t}}\right)\left(\frac{P_{t}}{P_{t+1}}\right)\left(\frac{\chi_{1 t+1}}{\chi_{1 t}}\right) C_{t+1}^{-\sigma} \\
\left(\frac{M_{t}}{P_{t}}\right)^{-\varsigma} / \chi_{1 t} C_{t}^{-\sigma}=\frac{R_{t}-1}{R_{t}}, \\
\frac{N_{t}(i)^{\mu}}{\chi_{1 t} C_{t}^{-\sigma}}=\frac{W_{t}(i)}{P_{t}}, \\
R_{t}=R_{t}^{*} \exp \left(-b N F A_{t}+\ln \chi_{2 t}\right) \frac{\varepsilon_{t+1}}{\varepsilon_{t}}
\end{gathered}
$$

Equation (2.25) is the Euler condition, representing the household's optimal intertemporal allocation of consumption given domestic nominal interest rate and price levels arising in the two time periods. It can also be written as

$$
\chi_{1 t} C_{t}{ }^{-\sigma} / \beta E_{t} \chi_{1 t+1} C_{t+1}{ }^{-\sigma}=R_{t} /\left(1+E_{t} \pi_{t+1}\right)
$$

which states that the marginal rate of substitution between consumption at $t$ and $t+1$ is equal to the real marginal rate of transformation in "production" - that is, the real return available when using domestic bonds to transfer consumption between $t$ and $t+1$. Here the inflation rate is written in terms of the consumer price index, $\pi_{t+1}$, and defined as $\pi_{t+1}=\left(P_{t+1}-P_{t}\right) / P_{t}$. Similarly $(2.26)$ is another version of the Euler condition,

$$
\chi_{1 t} C_{t}^{-\sigma} / \beta E_{t} \chi_{1 t+1} C_{t+1}^{-\sigma}=R_{t}^{*} \exp \left(-b N F A_{t}+\ln \chi_{2 t}\right) /\left(1+E_{t} \pi_{t+1}\right)
$$

where optimal intertemporal consumption requires the marginal rate of substitution between consumption at $t$ and $t+1$ is equal to the real marginal rate of transformation in "production" when using foreign bonds to transfer consumption between $t$ and $t+1$. 
Note that the risk premium term $\exp \left(-b N F A_{t}+\ln \chi_{2 t}\right)$ appears here because of our assumption of incomplete asset markets.

Equation (2.29), the well-known uncovered interest parity condition, is the implication of (2.25) and (2.26) holding simultaneously. Compared to the usual uncovered interest parity condition, (2.29) has one more term--the risk premium--that guarantees that asset market returns converge when the asset markets are incomplete.

Equation (2.27) represents the household's optimal money holding condition. This sets the marginal rate of substitution between real money balances and consumption equal to the opportunity cost of holding money (the money rate of interest) ${ }^{21}$. Equation (2.28) represents the intratemporal optimality condition between leisure and consumption, requiring the marginal rate of substitution between leisure and consumption to be set equal to the real wage. We will later demonstrate that because all firms have the same technology and hire the same types of labor inputs from competitive labor markets, the wage rate will be identical across all labor types so that the index $\mathrm{i}$ can be dropped from the wage rate in (2.28). Using that common wage rate, $W_{t},(2.28)$ can be written as

$$
\frac{N_{t}^{\mu}}{\chi_{1 t} C_{t}^{-\sigma}}=\frac{W_{t}}{P_{t}}
$$

In addition to these first order conditions and the model's initial conditions, the following transversality conditions must hold,

$$
\lim _{t \rightarrow \infty} \beta^{t} \lambda_{3 t} m_{t}=0, \lim _{t \rightarrow \infty} \beta^{t} \lambda_{3 t} B_{t} / P_{t} R_{t}^{t}=0
$$

\footnotetext{
${ }^{21}$ Because the return on the bond, $i_{t+1}=R_{t}-1$, is paid in period $\mathrm{t}+1$ rather than in period $\mathrm{t}$, the return needs to be discounted to the time of choice in period t, i.e. divided by $R_{t}$.
} 


$$
\text { and } \lim _{t \rightarrow \infty} \lambda_{3 t} \varepsilon_{t} B_{t}^{*} / P_{t}\left(R_{t}^{*} \exp \left(-b N F A_{t}+\ln \chi_{2 t}\right)\right)^{*}=0 \text {, }
$$

where $m_{t}=M_{t} / P_{t}$ is the level of real money balances.

\subsection{The Rest of the World}

To solve the domestic firm's optimization problem, it is first necessary to derive the demand for domestically produced goods arising from those consumers located in the rest of the world. To do this, we assume that the representative household in the rest of the world has the same preferences as domestic households. With this, we derive the following equations for foreign consumers (that are analogous to those derived earlier for home consumers),

$$
\begin{aligned}
& C_{F, t}^{*}(z)=\left(\frac{P_{F, t}^{*}(z)}{P_{F, t}{ }^{*}}\right)^{-\theta} C_{F, t}^{*}, \\
& C_{H, t}^{*}(i)=\left(\frac{P_{H, t}^{*}(i)}{P_{H, t}^{*}}\right)^{-\theta} C_{H, t}^{*},
\end{aligned}
$$

where $C_{F, t}{ }^{*}(z)$ and $C_{H, t}{ }^{*}(i)$ denote, respectively, the demand for foreign produced good $\mathrm{z}$ and good i imported from the small open economy by foreign households. $C_{F, t}{ }^{*}$ and $C_{H, t}{ }^{*}$ are the corresponding levels of the two composite consumption goods. Note that a star has been used to denote a foreign variable. For these variables, we find

$$
\begin{aligned}
& C_{F, t}^{*}=\left(1-\alpha^{*}\right)\left(\frac{P_{F, t^{*}}^{*}}{P_{t}^{*}}\right)^{-\eta} C_{t}^{*}, \\
& C_{H, t}^{*}=\alpha^{*}\left(\frac{P_{H, t}^{*}}{P_{t}^{*}}\right)^{-\eta} C_{t}^{*},
\end{aligned}
$$


where $\alpha^{*}$ denotes the share of home exported goods in foreign total consumption. Following Gali and Monacelli (2002), we assume that the rest of the world economy is much bigger in size than the home economy so that $\alpha^{*}$ is negligible. Then treating $\alpha^{*}$ as approximately zero, the foreign economy essentially becomes a closed economy with $C_{t}^{*} \approx Y_{t}^{*}$. In this case, equation (2.35) can be rewritten as

$$
C_{H, t}^{*}=\alpha^{*}\left(\frac{P_{H, t}^{*}}{P_{t}^{*}}\right)^{-\eta} Y_{t}^{*}
$$

Given our assumptions on preferences, the Euler equation for foreign households can be written in terms that are analogous to that derived for domestic consumption [in $(2.25)]$ as

$$
\left(C_{t}^{*}\right)^{-\sigma}=\beta\left(R_{t}^{*}\right) E_{t}\left(P_{t}^{*} / P_{t+1}^{*}\right)\left(\chi_{1 t+1}^{*} / \chi_{1 t}^{*}\right)\left(C_{t+1}^{*}\right)^{-\sigma}
$$

where $R_{t}^{*}$ is the gross return realized on bonds denominated in foreign currency. Recall that we assumed that the home countries domestic bonds cannot be traded internationally. Hence foreign households will hold only foreign bonds so that only this Euler equation is relevant to foreign households. For simplicity we assume that foreign households experience the same shock to their consumption, that is, $\chi_{1 t}=\chi_{1 t}{ }^{*}$, then we can rewrite the above equation to be

$$
\left(C_{t}^{*}\right)^{-\sigma}=\beta\left(R_{t}^{*}\right) E_{t}\left(P_{t}^{*} / P_{t+1}^{*}\right)\left(\chi_{1 t+1} / \chi_{1 t}\right)\left(C_{t+1}^{*}\right)^{-\sigma} .
$$

\subsection{Firm Behavior}

To model the supply side of our economy we assume that there is a continuum of firms indexed by $i \in[0,1]$ in the small open economy. All firms use the same technology 
to produce a similar but differentiated good. The common technology incorporates constant return to scale (for simplicity, capital is ignored) and is written as,

$$
Y_{t}(i)=A_{t} N_{t}(i)
$$

where $N_{t}(i)$ is the aggregate quantity of the labor employed by firm $\mathrm{i}$ and $A_{t}$ is an exogenous technology shock common to all firms. The shock is assumed to follow an AR (1) process,

$$
\ln A_{t}=\rho_{A} \ln A_{t-1}+\varepsilon_{A t}
$$

where $0<\rho_{A}<1$, and $\varepsilon_{A t}$ is a white noise disturbance that is assumed to be normally distributed, serially uncorrelated, with zero mean and standard deviation $\sigma_{\mathrm{A}}$.

Firm $i$ is assumed to hire labor in a competitive labor market but to be the sole seller of its differentiated product. This makes each firm a price taker in the input market and a price setter in its output market. The existence of market power, however, complicates our analysis because we eventually wish to evaluate the welfare consequences of alternative monetary policy rules through their effects on the representative household's utility function. Doing so requires the use of a linear approximation (to the utility function) that is valid only in the neighborhood of the social optimum (see Woodford, 2003). Hence to offset the distortion arising under monopolistic competition, we assume that the government uses a production subsidy $(\tau)$ set in such a way that in the steady state the net markup is zero (see Rotemberg and Woodford, 1997 and 1999, and Smets and Wouters, 2002). ${ }^{22}$

\footnotetext{
${ }^{22}$ Despite using a subsidy that is optimal from the point of view of the steady state subsidy rate, some overall distortion may arise period to period due to fact that the distortions arising from tax incidence and market power may be dependent on the size of the shocks realized each period. This period-specific distortion is assumed to be of second order.
} 
The firm then chooses the employment level, $N_{t}(i)$, and the selling price, $P_{H, t}^{n}(i)$, that will maximize its expected profits subject to its production technology and demand function. With the ability to recontract in the labor market each period, firm i will choose the quantity of $N_{t}(i)$ each period that minimizes the input cost of producing any level of output. That is,

$$
\min _{N_{t}(i)}\left(\frac{W_{t}(i)}{P_{H, t}}\right) N_{t}(i)+\varphi_{H, t}(i)\left(Y_{t}(i)-A_{t} N_{t}(i)\right)
$$

where the Lagrangian multiplier becomes, $\varphi_{H, t}(i)$, the firm's real marginal cost of production (inclusive of the subsidy). The first order condition for $N_{t}(i)$ is then $\varphi_{H, t}(i)=W_{t}(i) / A_{t} P_{H, t}$. Notice that because all firms exhibit the same constant returns to scale technology, the real marginal cost for each firm is the same and is independent of its individual output level. This, together with equal availability of labor supplies and perfectly competitive labor markets, means that we can rewrite the first order condition without each firm specific index and derive the following representation of each firm's marginal cost,

$$
\varphi_{H, t}=\frac{W_{t}}{A_{t} P_{H, t}}
$$

Turning next to the firm's optimal pricing decision, we add price stickiness by following Calvo (1983), as later adapted for price indexation by Christiano et al. (2001) ${ }^{23}$.

${ }^{23}$ Christiano et al. (2001) consider two specifications for how indexation can affect the firm that cannot reset its price each period. The first specification has $P_{i t}=\widetilde{\Pi} P_{i, t-1}$, where $\bar{\Pi}$ is the steady state, gross inflation rate (see also Erceg, Henderson and Levin, 2000 and Yun, 1996). Christiano et al refer to this case as static price updating. The second specification sets $P_{i t}=\Pi_{t-1} P_{i, t-1}$ and is called dynamic price updating. The latter specification is motivated in part by claims that the former does not generate sufficient 
In Calvo's model, a random fraction, $1-\omega$, of all firms will receive each period a signal that allows them to adjust their price. The remaining fraction, $\omega$, must keep their previously set price fixed. Christiano et al (2001) modify this to allow the fraction, $\omega$, who cannot reset their price this period to index it to last period's inflation rate. We assume that the degree to which these firms can index their price is $\delta$.

Under this price setting strategy, firm $i$ with the opportunity to set a new price this period will choose the price that solves the following maximization problem,

$$
\max _{P_{H, t}^{n}(i)} \sum_{k=0}^{\infty} \omega^{k} E_{t}\left(Q_{t, t+k}\right)\left[(1+\tau) P_{H, t}^{n}\left(X_{H, t k}\right)^{\delta} Y_{t+k}(i)-\varphi_{H, t+k} P_{H, t+k} Y_{t+k}(i)\right]
$$

subject to its demand function, $Y_{t+k}(i)=C_{H, t+k}(i)+C_{H, t+k}^{*}(i)$. Here the stochastic discount factor, $Q_{t, t+k}$, given in $(2.25)$ as $\beta^{k} E_{t}\left(P_{t} / P_{t+k}\right)\left(\chi_{1 t+k} / \chi_{1 t}\right)\left(C_{t+k} / C_{t}\right)^{-\sigma}$, is used to compute the real value in period $t$ of a unit of a good produced in period $t+k$.

The solution to (2.42) is the new price, $P_{H, t}^{n}(i)$, that will be chosen by firm $i$ when it gets the opportunity to adjust its price. Since all firms face the same cost and demand conditions, each will pick the same new price so that we can drop the individual subscript $i$ and write $P_{H, t}^{n}(i)=P_{H, t}^{n}$. In addition, we use $\left(X_{H, t k}\right)^{\delta}$ to stand for the price indexation rule where $X_{H, t k}=\frac{P_{H, t}}{P_{H, t-1}} \frac{P_{H, t+1}}{P_{H, t}} \frac{P_{H, t+2}}{P_{H, t+1}} \cdots \cdots \frac{P_{H, t+k-1}}{P_{H, t+k-2}}$ for $k \geq 1$, otherwise, $X_{H, t k}=1$ and where $\delta \in[0,1]$ denotes the degree of price indexation. Thus when $\delta=1$, all firms' prices are fully indexed and when $\delta=0$, no prices are indexed.

inertia in inflation (see Fuhrer and Moore, 1995, and Gali and Gertler, 1999). We adopt the second specification. 
Using (2.10) and (2.33) and incorporating price indexation, the demand functions for each $Y_{t+k}(i)$ can be written as a function of aggregate domestic output. That is

$$
Y_{t+k}(i)=\left(\frac{P_{H, t}^{n}\left(X_{H, t k}\right)^{\delta}}{P_{H, t+k}}\right)^{-\theta} Y_{t+k}
$$

where $P_{H, t+k}(i)=P_{H, t}^{n}(i) X_{H, t k}^{\delta}=P_{H, t}^{n} X_{H, t k}^{\delta}$ with probability $\omega^{k-t}$ and where aggregate output $Y_{t+k}=C_{H, t+k}+C_{H, t+k}^{*}$.

Substituting (2.43) into (2.42), and then differentiating (2.42) with respect to $P_{H, t}^{n}$ gives the following first order condition for an internal optimum,

$$
P_{H, t}^{n}=\left(\frac{\theta}{(\theta-1)(1+\tau)}\right) \frac{E_{t} \sum_{k=t}^{\infty}(\omega \beta)^{k-t} \chi_{t+k} C_{t+k}{ }^{-\sigma} Y_{t+k} P_{t+k}{ }^{-1} P_{H, t+k}{ }^{1+\theta} X_{H, t k}{ }^{-\delta \theta} \varphi_{H, t+k}}{E_{t} \sum_{k=t}^{\infty}(\omega \beta)^{k-t} \chi_{t+k} C_{t+k}{ }^{-\sigma} Y_{t+k} P_{t+k}{ }^{-1} P_{H, t+k}{ }^{\theta} X_{H, t k}{ }^{\delta(1-\theta)}}
$$

Equation (2.44) implies that the new nominal price picked by firm $i$ in period $t$ will be a markup, $\theta /(\theta-1)$, over expected future nominal marginal cost. Because the firm is forward looking (given the previous pricing decisions made by the firm's rivals as embodied in $\left.P_{H, t-1}\right)$, optimal price setting requires an inflation forecast and a set of predetermined prices to inform the firm's current price decision.

In the special case where $\omega=0$, all firms can adjust their price each period. This implies that prices are perfectly flexible so that (2.44) collapses to

$$
\frac{P_{H, t}^{n}}{P_{H, t}}=\left(\frac{\theta}{(\theta-1)(1+\tau)}\right) \varphi_{H, t}
$$

In each period each firm sets its new money price so that its relative price is equal to a constant markup (inclusive of the subsidy) over its real marginal cost. This is 
standard optimizing behavior under the monopolistic competition. Under the assumption that the optimal subsidy is chosen by the government, $\tau=[\theta /(\theta-1)]-1$ so that $P_{H, t}^{n} / P_{H, t}=\varphi_{H, t}$, i.e., price equals marginal cost.

Applying the optimal subsidy to the general case we find

$$
P_{H, t}^{n}=\frac{E_{t} \sum_{k=t}^{\infty}(\omega \beta)^{k-t} \chi_{t+k} C_{t+k}{ }^{-\sigma} Y_{t+k} P_{t+k}{ }^{-1} P_{H, t+k}{ }^{1+\theta} X_{H, t k}{ }^{-\delta \theta} \varphi_{H, t+k}}{E_{t} \sum_{k=t}^{\infty}(\omega \beta)^{k-t} \chi_{t+k} C_{t+k}{ }^{-\sigma} Y_{t+k} P_{t+k}{ }^{-1} P_{H, t+k}{ }^{\theta} X_{H, t k}{ }^{\delta(1-\theta)}}
$$

\subsection{Import Pricing Behavior}

In a small open economy when assets markets are incomplete and inhabitants cannot insure themselves perfectly from foreign shocks, the exchange rate (nominal, real or both) becomes an important endogenous variable and one that the monetary authority may need to take into account when setting policy. To understand the exchange rate's impact on the open economy one needs to know exactly how changes in the exchange rate will affect the domestic price of imports, that is, how exchange rate changes passthrough into domestic prices. In the literature there are two extreme hypotheses. First, foreign producers (exporters) could set their export good price optimally in terms of their own (foreign) currency and then simply translate set the small open economy price into domestic currency by the exchange rate. Here the import good price in the domestic market is set as $P_{F, t}(z)=\varepsilon_{t} P_{F, t}{ }^{*}(z)$. This is called producer currency pricing (PCP) and implies that the exchange rate elasticity is one and exchange rate pass-through is complete in the short run. On the other hand, foreign producers could set their export good price directly in terms domestic currency in relation to the demand conditions faced 
there. This is called local currency pricing, LCP, (or pricing to market, PTM,) and implies that in the short run, the elasticity of the foreign export good price with respect to the exchange rate will be zero.

Note that while the degree of exchange rate pass-through into domestic price is to some extent related to pricing method used by the firm, it is not the only factor. Another key determinant of the degree of exchange rate pass through is whether there are costs of adjustment that lead to sticky prices in the destination market. For example, even if the foreign exporter used PCP in setting its price, but the domestic economy had a bylaw requiring all firms (domestic or foreign firms) to keep their prices fixed for one entire period, then the elasticity of the import price with respect to the exchange rate in the short run would be zero rather than one under PCP. Similarly if the foreign exporter used LCP to set its price, but the domestic economy required all firms to reset their price each period, then the elasticity of price with respect to the exchange rate in the short run would be one rather than zero even under LCP pricing. This is because the foreign firm will set its price ex ante to satisfy the law of one price even when using LCP. In this sense it is misleading to directly relate PCP and LCP to the exchange rate pass-through without recognizing the imposition of additional conditions. ${ }^{24}$ Strictly speaking, PCP in combination with local currency flexible prices results in complete exchange rate passthrough, while LCP in combination with local currency price stickiness is needed to generate incomplete (zero in the limit) exchange rate pass-through (see Lane, 2001). As

\footnotetext{
${ }^{24}$ Lane (2001) argues that because local-currency sticky prices or destination market rigidities are a key ingredient in incomplete pass-through, LCP is a mislabeling since LCP refers strictly to the ability of firms to choose optimally different prices for different markets. However, the term is now commonly used in the literature.
} 
might be expected, the empirical evidence suggests that actual exchange rate pass through will fall somewhere between zero and one. ${ }^{25}$

To model incomplete pass-through, we consider a representative importer who imports a representative differentiated import good $z$ at the cost $\varepsilon_{t} P_{F_{2},}{ }^{*}(z)$. The importer is then allowed to change the domestic price if it wishes, but can do so only by incurring a quadratic adjustment cost. Following Rotemburg (1982) and Laxton and Pesenti (2003), the adjustment cost (per dollar sold) is defined as

$$
A C_{M, t}=\frac{\omega_{M}}{2}\left(\frac{P_{F, t}{ }^{n}(z) / P_{F, t-1}(z)}{\left(P_{F, t-1} / P_{F, t-2}\right)^{\delta}}-1\right)^{2}
$$

where $\omega_{M} \geq 0$, scales the magnitude of the price adjustment cost as a function of its rate of change. In (2.46), the adjustment cost rises as the importer's specific inflation rate rises relative to some fraction, $\delta$, of the past inflation rate experienced by the import good sector as a whole. Here the $\delta$ allows for some quasi-automatic price adjustment process equivalent to the feature of indexation allowed for under Calvo pricing. In terms of its contribution to the analysis, the $\delta$ allows the model to capture the nominal inertia appearing empirically in inflation dynamics. ${ }^{26}$ Lastly, just as was the case for domestic

\footnotetext{
${ }^{25}$ See Goldberg and Knetter (1997) who find that the degree of pass-through in international prices over a year is typically around 0.5. Campa and Goldberg (2002) estimate exchange rate pass-through for a range of OECD countries using the simple versions of PCP and LCP hypothesis and find that degree of passthrough is less than one but greater than zero in the short run and becomes complete only in the long-run. Choudhri et al (2005) develop a small economy model that nests PCP and LCP as special cases and estimate the degree of exchange rate pass-through for five price indexes for non-U.S. G-countries. They find that exchange rate pass-through to consumer prices and wages is low in the short run and near zero in the first quarter. The pass-through to export and import prices is larger but still incomplete in the short run. The first quarter values range from about a fourth for export prices to roughly a half for import prices. ${ }^{26}$ The adjustment cost can be specified differently. For example, a variant of this specification relates changes in the importer's price inflation rate to a steady state gross inflation rate, $\Pi \geq 1$ instead of the last period of inflation rate, $A C_{M, t}=\omega_{M 1}\left(P_{F, t}(z) / \Pi P_{F, t-1}(z)-1\right)^{2} / 2$, and alternative versions add both the steady state $\Pi$ and the lagged inflation rate to the quadratic adjustment cost term,
} 
goods, the fact that the importer has market power means that the government will set an optimal subsidy, $\tau_{M}$, to induce the efficient level of output. Then because we have assumed that the elasticities of substitution among each variety of domestic and import goods are both equal to $\theta$, the optimal subsidy rates will also be equal. That is, $\tau=\tau_{M}=1 /(\theta-1)$

Hence facing this price adjustment cost, the representative importer will set its price $P_{F, t}{ }^{n}(z)$ by solving the following maximization problem subject to the downward sloping demand function that it faces,

$$
\max E_{t} \sum_{k=0}^{\infty} Q_{t, t+k}\left[\left(1+\tau_{M}\right) P_{F, t+k}{ }^{n}(z)-\varepsilon_{t} P_{F, t+k}{ }^{*}(z)\right] C_{F, t+k}(z)-A C_{M, t+k} P_{F, t+k} C_{F, t+k}
$$

where

$$
C_{F, t}(z)=\left(\frac{P_{F, t}{ }^{n}(z)}{P_{F, t}}\right)^{-\theta} C_{F, t}
$$

The first order condition for $P_{F, t}{ }^{n}(z)$ is

$$
\begin{aligned}
& (1-\theta)\left(1+\tau_{M}\right)\left(\frac{P_{F, t}(z)}{P_{F, t}}\right)^{-\theta} C_{F, t}+\theta\left(\frac{P_{F, t}(z)}{P_{F, t}}\right)^{-\theta-1} \frac{\varepsilon_{t} P_{F, t}{ }^{*}(z)}{P_{F, t}} C_{F, t} \\
& -\omega_{M}\left(\frac{P_{F, t}(z) / P_{F, t-1}(z)}{\left(P_{F, t-1} / P_{F, t-2}\right)^{\delta}}-1\right)\left(\frac{1 / P_{F, t-1}(z)}{\left(P_{F, t-1} / P_{F, t-2}\right)^{\delta}}\right) P_{F, t} C_{F, t} \\
& +\omega_{M} \beta \frac{P_{t}}{P_{t+1}} \frac{\chi_{1, t+1}}{\chi_{1, t}}\left(\frac{C_{t+1}}{C_{t}}\right)^{-\sigma}\left(\frac{P_{F, t+1}(z) / P_{F, t}(z)}{\left(P_{F, t} / P_{F, t-1}\right)^{\delta}}-1\right)\left(\frac{P_{F, t+1}(z) /\left(P_{F, t}(z)\right)^{2}}{\left(P_{F, t-1} / P_{F, t-2}\right)^{\delta}}\right) P_{F, t+1} C_{F, t+1} \\
& =0
\end{aligned}
$$

$A C_{M, t}=\omega_{M 1}\left(P_{F, t}(z) / \Pi P_{F, t-1}(z)-1\right)^{2} / 2+\omega_{M 2}\left(\left[P_{F, t}(z) / P_{F, t-1}(z)\right] /\left(P_{F, t-1} / P_{F, t-2}\right)-1\right)^{2} / 2$. See Ireland 2001 and 2003 for details. 
Note that our use of a representative importer implies that all retailers will be identical and hence make exactly same decision in a symmetric equilibrium. The following relationship must then hold in equilibrium, $P_{F, t}(z)=P_{F, t}$. Using this, the first order condition above simplifies to

$$
\begin{aligned}
& (1-\theta)\left(1+\tau_{M}\right)+\theta \frac{\varepsilon_{t} P_{F, t}{ }^{*}(z)}{P_{F, t}}-\omega_{M}\left(\frac{\Pi_{F, t}}{\left(\Pi_{F, t-1}\right)^{\delta}}-1\right)\left(\frac{\Pi_{F, t}}{\left(\Pi_{F, t-1}\right)^{\delta}}\right), \\
& +\omega_{M} \beta \frac{P_{t}}{P_{t+1}} \frac{\chi_{1, t+1}}{\chi_{1, t}}\left(\frac{C_{t+1}}{C_{t}}\right)^{-\sigma}\left(\frac{\Pi_{F, t+1}}{\left(\Pi_{F, t}\right)^{\delta}}-1\right)\left(\frac{\left(\Pi_{F, t+1}\right)^{2}}{\left(\Pi_{F, t}\right)^{\delta}}\right) \frac{C_{F, t+1}}{C_{F, t}}=0
\end{aligned}
$$

where $\Pi_{F, t}=P_{F, t} / P_{F, t-1}$ denotes the gross inflation rate of import prices and where symmetry again produces $\Pi_{F, t}(z)=\Pi_{F, t}$. In the special case where $\omega_{M}=0$, collapses to

$$
\left(1+\tau_{M}\right) P_{F, t}(z)=\frac{\theta}{\theta-1} \varepsilon_{t} P_{F, t}{ }^{*}(z) \text { and implies } P_{F, t}(z)=\varepsilon_{t} P_{F, t}{ }^{*}(z)
$$

when the optimal subsidy is set.

\subsection{Steady State Equilibrium}

The steady-state equilibrium is the equilibrium that will arise in the absence of all shocks when all prices are completely flexible and when the exogenous variables, including foreign output, price and interest rate are given as constants (or change at a given constant rate). Here we focus on the special case of a steady-state inflation rate of zero. Given that the government imposes optimal output subsidies and in the absence of any other distortions, our steady-state will correspond to a social optimum. It is about these optimal steady state values that we log-linearize the model to describe its motion 
out of equilibrium. To designate the steady state value of a variable, we use a bar over the variable and drop the time subscript.

In the steady state, all variables such as consumption, output, real balances, etc., must satisfy: the first-order conditions for the representative household; the profitmaximizing conditions for the firm and importers; the various budget constraints faced by the household, firm and government; and the market clearing conditions.

Beginning with the government budget constraint in (2.23), a zero inflation steady state implies that the money stock will be constant in the steady state. Using our earlier finding that $\tau=\tau_{m},(2.23)$ can now be rewritten as $\tau \bar{P} \bar{Y}+\tau \bar{P}_{F} \bar{C}_{F}=\bar{M}-\bar{M}+\bar{T}$ or $\tau \bar{P} \bar{Y}+\tau \bar{P}_{F} \bar{C}_{F}=\bar{T}$. The subsidy paid to firms and importers in the steady state exactly equals the lump-sum tax revenue collected from household.

Next we turn to the representative household's budget constraint in (2.21) and express it in the steady state as

$$
\begin{aligned}
& \bar{P} \bar{C}+\bar{M}+(1 /(1+\bar{i})) \bar{B}+\bar{\varepsilon}^{*}\left(1 /\left(1+\bar{i}^{*}\right) \exp \left(-b \overline{N F A}+\ln \bar{\chi}_{2}\right)\right)+\bar{T} \\
& =\int_{0}^{1} \bar{N}(i) \bar{W}(i) d i+\int_{0}^{1} \bar{D}(i) d i+\int_{0}^{1} \bar{D}(z) d z+\bar{M}+\bar{B}+\bar{\varepsilon} \bar{B}^{*}
\end{aligned}
$$

where $1+\vec{i}=\bar{R}^{27}$, and $1+\bar{i}^{*}=\bar{R}^{*}$. Integrating over all individuals and simplifying, yields

$$
\begin{aligned}
& \bar{P} \bar{C}-\bar{P}_{H} \bar{Y}=(\bar{i} /(1+\bar{i})) \bar{B}+\tau \bar{P}_{H} \bar{Y}+\tau \bar{P}_{F} \bar{C}_{F}-\bar{T} \\
& -\bar{\varepsilon} \bar{B}^{*}\left(1 /\left(1+\bar{i}^{*}\right) \exp \left(-b \overline{N F A}+\ln \bar{\chi}_{2}\right)\right)+\bar{\varepsilon} \bar{B}^{*}
\end{aligned}
$$

\footnotetext{
${ }^{27}$ It will be shown shortly that in the steady state the interest rate $\bar{i}$ will equal $(1-\beta) / \beta$.
} 
where we have simplified (2.49), using the condition that the use of government subsidies to induce production efficiency implies that price equals marginal cost in the steady state so that

$$
\begin{aligned}
& \int_{0}^{1}\left(\int_{0}^{1} \bar{N}(i) \bar{W}(i) d i\right) d i=\int_{0}^{1}\left(\int_{0}^{1} \bar{P}_{H}(i) \bar{Y}(i) d i\right) d i=\bar{P}_{H} \bar{Y}, \quad \text { with } \\
& \int_{0}^{1} \bar{D}(i) d i=\tau \overline{P_{H}} \bar{Y}, \quad \text { and } \int_{0}^{1} \bar{D}(z) d z=\tau \bar{P}_{F} \bar{C}_{F} .
\end{aligned}
$$

Equation (2.49) can be further simplified by noting first that the government budget constraint implies that $\tau \bar{P} \bar{Y}+\tau \bar{P}_{F} \bar{C}_{F}-\bar{T}=0$. Next since domestic bonds cannot be traded internationally, $\bar{B}=0$ in the steady state. ${ }^{28}$ Finally, with the same production technology $P_{t}, P_{H, t}$, and $P_{F, t}$ all become equal in the steady state so that the steady state values of the terms of trade, $s_{t}$, the nominal exchange rate, $\varepsilon_{t}$, and the real exchange rate, $q_{t}$, are all equal to one. Using all these conditions, (2.49) then reduces to

$$
\bar{P}(\bar{C}-\bar{Y})=-\bar{\varepsilon} \bar{B}^{*}\left(1 /\left(1+\bar{i}^{*}\right) \exp (-b \overline{N F A})\right)+\bar{\varepsilon} \bar{B}^{*}
$$

where $\ln \bar{\chi}_{2}=0$.

If we use EX to represent exports and IM imports, the national income identity for any small open economy can be written as $Y=C+I+G+E X-I M$. Because our model has neither investment, $I$, nor government expenditure, $G$, the identity reduces in our case to $Y-C=E X-I M=C A$, where $C A$ denotes the size of the current account and $C A>0$ implies that the $\mathrm{CA}$ is in surplus. When the country sells more to the rest of the world than it purchases, the home country will accumulate assets from the rest of the world. By assumption, our domestic households do not hold foreign currencies, so the

${ }^{28}$ Even more strongly, since households behave identically, net domestic bonds must be zero each period so that $B_{t}=0$. 
capital account deficit that is required to offset the current account surplus corresponds to the domestic country's accumulation of foreign bonds. It follows, then, that $(2.50)$ reflects the steady state version of the balance of payments condition that must hold for this economy at each point time.

From (2.50) it is apparent that for $\bar{C}=\bar{Y}, N F A_{t}$ must be zero in the steady state. At present, however, the analysis does not seem to have enough structure to require either $\bar{C}=\bar{Y}$ or $\overline{N F A}=0$. In the paragraph below we illustrate how the earlier modeling assumptions do imply the additional constraints needed to generate this special steady state result.

In the steady state, consumption must remain constant. Therefore, from the Euler condition in (2.25) for the household's optimal intertemporal allocation of consumption, we see that in the steady state $\bar{C}^{-\sigma}=\beta(1+\bar{i})(1 / 1+\bar{\pi}) \bar{C}^{-\sigma}$, where $\bar{\chi}_{1}=1$. Because our steady state is one in which $\bar{\pi}=0$, the steady state value of the nominal (and real) interest rate then becomes $\bar{i}=(1-\beta) / \beta$ or $\bar{R}=1 / \beta$. That is, without capital in the model, the steady state rate of interest is determined by time preference alone. Next, if we use the Euler equation for the rest of the world (from (2.37)), we can see that in its zero inflation steady state that $\bar{i}^{*}=\left(1-\beta^{*}\right) / \beta^{*}$ or $\bar{R}^{*}=1 / \beta^{*}$. If we now use the alternative domestic Euler equation from (2.26) that permits domestic households to borrow and lend the foreign bond, we obtain the steady state relationship, $\exp (-b \overline{N F A})=1 / \beta \bar{R}^{*}$. This illustrates that if $\beta \bar{R}^{*}=1$, then $\overline{N F A}=0$; otherwise $\overrightarrow{N F A} \neq 0$. Lastly if we link together the steady state versions of the three Euler equations immediately above, we can also see that if $\beta=\beta^{*}$, then $\bar{R}=1 / \beta=1 / \beta^{*}=\bar{R}^{*}$, 
and $\overline{N F A}=0$. It follows that our earlier assumption that foreign households have the same utility function as domestic households (except for the domestic taste shock) imposes $\beta=\beta^{*}$ and so implies $\overline{N F A}=0$ and hence $\bar{C}=\bar{Y}$ as a steady state condition. Otherwise, the position of NFB in the steady state will be positive or negative depending on whether we begin with $\bar{R}=1 / \beta$ greater or less than $\bar{R}^{*}=1 / \beta^{*}$. The assumption that $\beta=\beta^{*}$ is made for convenience so that $N F A_{t}=0$ in the steady state.

The optimality condition for money holdings relative to consumption in (2.27) can now be written for the steady state as $\bar{m}^{-\zeta}=\bar{C}^{-\sigma}(\bar{i} / 1+\bar{i})$. Using $\bar{i}=(1-\beta) / \beta$ this implies that

$$
\bar{m}=\frac{\bar{M}}{\bar{P}}=\left(\frac{1}{(1-\beta)} \bar{Y}^{\sigma}\right)^{1 / \varsigma} .
$$

Equation (2.51) states that real money balances in steady state are positively related to the steady-state output level and negatively related to the opportunity cost of holding money. Note that while money is neutral in the steady state, $\bar{M}$ and $\bar{P}$ are not separately determined by (2.51). Given one, the other is determined.

The steady state condition for the optimal leisure and consumption tradeoff is given by equation (2.28), that is, $\bar{N}(i)^{\mu} / \bar{C}^{-\sigma}=\bar{W}(i) / P . \quad$ Then using $Y_{t}=Y_{t}(i)\left(P_{H, t}(i) / P_{H, t}\right)^{\theta}$ in the production function we can obtain $\bar{N}(i)=\left(\bar{P}_{H}(i) / \bar{P}_{H}\right)^{\theta} \bar{Y} / \bar{A}=\bar{Y} / \bar{A}$, where the latter equality arises since all firms will pick the same price in the steady state so that $\bar{P}_{H}(i)=\bar{P}_{H}$. Next we can use $(2.41)$ to get $\bar{\varphi}_{H}(i) \bar{A}=\bar{W}(i) / \bar{P}_{H}$. As argued before the index i can be dropped from the real marginal 
cost, i.e., $\bar{\varphi}_{H}(i)=\bar{\varphi}_{H}$, since all firms face identical production functions and market conditions. Then using (2.45) which will hold in the steady state, real marginal cost can be written as $\bar{\varphi}_{H}=[(1+\tau)(\theta-1) / \theta]\left(\bar{P}_{H}^{n} / \bar{P}_{H}\right)$. Given our assumptions about the form of the productivity shock, $\bar{A}=1$. Combining all these relationships, the intratemporal optimality condition for leisure and consumption in the steady state can be written as

$$
\bar{C}^{\sigma}\left(\frac{\bar{Y}}{\bar{A}}\right)^{\mu}=\bar{\varphi}_{H} \bar{A} \frac{\bar{P}_{H}}{\bar{P}}=\bar{A}\left(\frac{(1+\tau)(\theta-1)}{\theta}\right)\left(\frac{\bar{P}_{H}^{n}}{\bar{P}_{H}}\right)\left(\frac{\bar{P}_{H}}{\bar{P}}\right) .
$$

Using $\bar{C}=\bar{Y}, \bar{A}=1$, and $\bar{P}_{H}{ }^{n}=\bar{P}_{H}=\bar{P}$, it can be further to become

$$
\bar{Y}=\left(\frac{(1+\tau)(\theta-1)}{\theta}\right)^{\frac{1}{\sigma+\mu}}
$$

Equation (2.52) indicates that output level in steady state is affected by the following factors. First, increases in the degree of producer market power would decrease output further below the competitive level. That is, absent subsidies, the socially optimal level is approached only as $\theta$ goes to infinity. Second, in our model the steady state output is affected by the size of the output subsidy received by firms. When $\tau$ is set optimally so that $(1+\tau)(\theta-1) / \theta=1$, the subsidy counters fully the degree of monopoly power existing in the steady state and the level of output in steady state equals 1 . Finally, steady state output is also affected by the elasticity of marginal disutility of labor, $\mu$, and the intertemporal elasticity of substitution in consumption, $1 / \sigma$. Invoking the assumption of the optimal subsidy made earlier, we find that $\bar{Y}=1$. 


\subsection{The New Keynesian Phillips Curve}

Returning to equation (2.44), we must now recognize that only the fraction $1-\omega$ of all firms have the opportunity to set a new price decision at time $t$. The remainders have their previous price indexed to the inflation rate. This means that the domestic price level index will evolve according to the following indexation rule,

$$
P_{H, t}^{1-\theta}=(1-\omega)\left(P_{H, t}^{n}\right)^{1-\theta}+\omega\left(P_{H, t-1}\right)^{1-\theta}\left(\frac{P_{H, t-1}}{P_{H, t-2}}\right)^{\delta(1-\theta)}
$$

Dividing this by $\left(P_{H, t}\right)^{1-\theta}$, we obtain

$$
1=(1-\omega)\left(\frac{P_{H, t}^{n}}{P_{H_{s}, t}}\right)^{1-\theta}+\omega\left(\frac{P_{H, t-1}}{P_{H, t-2}}\right)^{\mathcal{\delta}(1-\theta)}\left(\frac{P_{H, t}}{P_{H, t-1}}\right)^{\theta-1}
$$

Defining $G_{t} \equiv P_{H, t}^{n} / P_{H, t}$ and log-linearizing this relative price around the zero inflation rate steady-state, we derive the following expression,

$$
(1-\omega)(\bar{G})^{1-\theta} \hat{G}_{t}=\omega \Pi^{(1-\theta)(\delta-1)}\left(\hat{\Pi}_{H, t}-\delta \hat{\Pi}_{H, t-1}\right)
$$

where $\hat{G}_{t}$ stands for the $\log$ deviation of $G_{t}$ from its steady-state value, $\bar{G}$, i.e., $\hat{G}_{t}=\log G_{t} / \bar{G}$. Since $\widetilde{G}=1$ and $\Pi=1$ under the zero steady state inflation condition, this expression can be simplified to

$$
\hat{G}_{t}=\frac{\omega}{1-\omega}\left(\hat{\Pi}_{H, t}-\delta \hat{\Pi}_{H, t-1}\right)
$$

where $\hat{\Pi}_{H, t}=\log \left(\Pi_{H, t} / \widehat{\Pi}\right)=\log \Pi_{H, t}=\log \left(P_{H, t} / P_{H, t-1}\right)=\hat{P}_{H, t}-\hat{P}_{H, t-1}$ is log deviation of gross inflation rate from its steady state. To approximate (2.44) around the zero inflation steady state, we first rewrite (2.44) as 


$$
\begin{aligned}
& E_{t} \sum_{k=0}^{\infty}(\omega \beta)^{k} \chi_{t+k} C_{t+k}^{-\sigma} Y_{t+k} G_{t}\left(\frac{P_{t+k}}{P_{t}}\right)^{-1}\left(\frac{P_{H, t+k}}{P_{H, t}}\right)^{\theta}\left(X_{H, t k}\right)^{\delta(1-\theta)} \\
& =\frac{\theta}{(\theta-1)(1-\tau)} E_{t} \sum_{k=0}^{\infty}(\omega \beta)^{k} \chi_{t+k} C_{t+k}^{-\sigma} Y_{t+k}\left(\frac{P_{t+k}}{P_{t}}\right)^{-1}\left(\frac{P_{H, t+k}}{P_{H, t}}\right)^{\theta+1}\left(X_{H, t k}\right)^{-\delta \theta} \varphi_{H, t+k}
\end{aligned}
$$

Then first log-linearizing the left-hand side of (2.54) around the steady-state values, we obtain

$$
\begin{aligned}
& E_{t} \sum_{k=0}^{\infty}(\omega \beta)^{k} \bar{\chi} \bar{C}^{-\sigma} \overline{Y G}\left(\frac{\bar{P}}{\bar{P}}\right)^{-1}\left(\frac{\bar{P}_{H}}{\bar{P}_{H}}\right)^{\theta}\left(\bar{X}_{H}\right)^{\delta(1-\theta)} \\
& {\left[\begin{array}{l}
1+\hat{\chi}_{t+k}-\sigma \hat{C}_{t+k}+\hat{Y}_{t+k}+\hat{G}_{t}-\left(\hat{P}_{t+k}-\hat{P}_{t}\right) \\
+\theta\left(\hat{P}_{H, t+k}-\hat{P}_{H, t}\right)+\delta(1-\theta)\left(\hat{P}_{H, t+k-1}-\hat{P}_{H, t-1}\right)
\end{array}\right]}
\end{aligned}
$$

Next $\log$-linearizing the right-hand side of (2.54) similarly yields

$$
\begin{aligned}
& \frac{\theta}{(\theta-1)(1-\tau)} E_{t} \sum_{k=0}^{\infty}(\omega \beta)^{k} \bar{\chi} \bar{C}^{-\sigma} \bar{Y}\left(\frac{\bar{P}}{\bar{P}}\right)^{-1}\left(\frac{\bar{P}_{H}}{\bar{P}_{H}}\right)^{\theta+1}\left(\bar{X}_{H}\right)^{-\delta \theta} \bar{\varphi}_{H} \\
& {\left[\begin{array}{l}
1+\hat{\chi}_{t+k}-\sigma \hat{C}_{t+k}+\hat{Y}_{t+k}-\left(\hat{P}_{t+k}-\hat{P}_{t}\right)+(\theta+1)\left(\hat{P}_{H, t+k}-\hat{P}_{H, t}\right) \\
-\delta \theta\left(\hat{P}_{H, t+k-1}-\hat{P}_{H, t-1}\right)+\hat{\varphi}_{H, t+k}
\end{array}\right]}
\end{aligned}
$$

Equating the above two equations, and canceling the terms that appear on both sides gives

$$
\hat{G}_{t}-\delta \hat{P}_{H, t-1}+\hat{P}_{H, t}=(1-\omega \beta) E_{t} \sum_{k=0}^{\infty}(\omega \beta)^{k}\left(\hat{\varphi}_{H, t+k}+\hat{P}_{H, t+k}-\delta \hat{P}_{H, t+k-1}\right)
$$

This equation can be rewritten as

$$
\hat{G}_{t}-\delta \hat{P}_{H, t-1}+\hat{P}_{H, t}=(1-\omega \beta)\left(\hat{\varphi}_{H, t}+\hat{P}_{H, t}-\delta \hat{P}_{H, t-1}\right)+\omega \beta\left(\hat{G}_{t+1}-\delta \hat{P}_{H, t}+\hat{P}_{H, t+1}\right) .
$$

Substituting (2.53) into it, one obtains the New Keynesian Phillip's curve,

$$
\hat{\Pi}_{H, t}=\frac{\delta}{1+\beta \delta} \hat{\Pi}_{H, t-1}+\frac{\beta}{1+\beta \delta} E_{t} \hat{\Pi}_{H, t+1}+\gamma \hat{\varphi}_{H, t}
$$


where $\gamma=\frac{(1-\omega)(1-\omega \beta)}{\omega(1+\beta \delta)}$. Note that $\varphi_{H, t}$ is marginal cost defined in terms of the price index $P_{H, t}$ that corresponds to the bundle of domestically produced goods.

Equation (2.55) indicates that (the log deviation of) domestic inflation depends on both past and expected future inflation rates and the current real marginal cost. ${ }^{29}$ The latter is itself a function of output, foreign output, productivity shock, and the preference shock to the disutility of work. When indexation is not possible so that $\delta=0$, the current Phillips curve reverts to the purely forward-looking Phillips curve standard in the literature, in which current inflation depends only on expected future inflation and current real marginal cost so that domestic inflation is independent of its history. Then as $\delta$ rises above zero to approach one, the weight given to past inflation rises and the weight given to expected future inflation falls. In this sense, the degree of indexation determines how backward looking the inflation process is. Finally, the effect of the real marginal cost on inflation mainly depends on the degree of price stickiness. In the extreme case where the price is completely fixed, i.e., $\omega=1$, then $\gamma=0$ and current real marginal cost has no effect on the current inflation rate at all.

If we now log-linearize import prices in (2.47) around their steady state value, we find that the inflation rate adjustment process for import good prices has the same general form as that derived for domestic good price inflation above. That is,

\footnotetext{
${ }^{29}$ Equation (2.57) is written in terms of the log deviation from the steady state, but since the steady state rate of inflation is assumed to be equal to zero, $\hat{\Pi}_{H, t}=\pi_{H, t}=\left(P_{H, t}-P_{H, t-1}\right) / P_{H, t-1}$ and $\hat{\Pi}_{H, t-1}=\pi_{H_{0} t-1}$ etc. Note that when the steady state rate of inflation is not zero, the log deviation is not the same thing as the actual inflation rate.
} 


$$
\hat{\Pi}_{F, t}=\frac{\delta}{1+\beta \delta} \hat{\Pi}_{F, t-1}+\frac{\beta}{1+\beta \delta} E_{t} \hat{\Pi}_{F, t+1}+\frac{\theta-1}{\omega_{M}(1+\beta \delta)}\left(\hat{\varepsilon}_{t}+\hat{P}_{F, t}^{*}-\hat{P}_{F, t}\right)
$$

Note that the lagged term, $\hat{\Pi}_{F, t-1}$, appears because our specification of the adjustment cost incorporates the past inflation rate. Hence when $\delta=0$, the adjustment cost becomes $A C_{M, t}=\omega_{M}\left(P_{F, t}(z) / P_{F, t-1}(z)-1\right)^{2} / 2$ and the lagged inflation term will disappear. Finally, the last term in (2.56) captures incomplete pass-through as the $\log$ deviation of (the domestic price equivalent of) the world price from the actual domestic price of imports. Following Monacelli (2003), we call this the law of one price gap. If we then define $\Psi_{t}=\varepsilon_{t} P_{F, t}{ }^{*} / P_{F, t}$ so that $\hat{\Psi}_{t}=\hat{\varepsilon}_{t}+\hat{P}_{F, t}{ }^{*}-\hat{P}_{F, t}$, then (2.56) can be rewritten as

$$
\hat{\Pi}_{F, t}=\frac{\delta}{1+\beta \delta} \hat{\Pi}_{F, t-1}+\frac{\beta}{1+\beta \delta} E_{t} \hat{\Pi}_{F, t+1}+\frac{\theta-1}{\omega_{M}(1+\beta \delta)} \hat{\Psi}_{t}
$$

\subsection{The IS Equation}

To derive the IS equation we begin with the market clearing condition in the goods markets. Because part of the output produced by domestic firms is allocated to the home consumers and part is exported to the rest of the world, the condition for market clearing in good market $i$ is $Y_{t}(i)=C_{H, t}(i)+C_{H, t}^{*}(i)$. Applying (2.10), (2.18), (2.33) and (2.36), $Y_{t}(i)$ can be written as

$$
Y_{t}(i)=\left(\frac{P_{H, t}(i)}{P_{H, t}}\right)^{-\theta}\left[(1-\alpha)\left(\frac{P_{H, t}}{P_{t}}\right)^{-\eta} C_{t}+\alpha^{*}\left(\frac{P_{H, t}}{\varepsilon_{t} P_{t}^{*}}\right)^{-\eta} C_{t}^{*}\right]
$$

Equation (2.58) expresses $Y_{t}(i)$ as a function of $C_{t}$ and $C_{t}{ }^{*}$, where we have used the assumption that domestic exporters use producer currency pricing (PCP) so that in 
foreign currency the law of one price holds, that is, $P_{H, t}(i)=\varepsilon_{t} P_{H, t}(i)^{*}$. To further simplify (2.58) we next use the definition, $Y_{t}=\left[\int_{0}^{1}\left(Y_{t}(i)\right)^{(\theta-1) / \theta} d i\right]^{\theta /(\theta-1)}$. Plugging $(2.58)$ into this definition of $Y_{t}$ yields

$$
Y_{t}=\left[(1-\alpha)\left(\frac{P_{H, t}}{P_{t}}\right)^{-\eta} C_{t}+\alpha^{*}\left(\frac{P_{H, t}}{\varepsilon_{t} P_{t}^{*}}\right)^{-\eta} C_{t}^{*}\right]\left[\int_{0}^{1}\left(\frac{P_{H, t}(i)}{P_{H, t}}\right)^{-(\theta-1)} d i\right]^{\frac{\theta-1}{\theta}}
$$

Applying the definition of $P_{H, t}$, that is, $P_{H, t}=\left[\int_{0}^{1} P_{H, t}(i)^{1-\theta} d i\right]^{1 /(1-\theta)}$, equation (2.59) can be written as ${ }^{30}$

$$
Y_{t}=(1-\alpha)\left(\frac{P_{H, t}}{P_{t}}\right)^{-\eta} C_{t}+\alpha^{*}\left(\frac{P_{H, t}}{\varepsilon_{t} P_{t}^{*}}\right)^{-\eta} C_{t}^{*}
$$

As was the case for the domestic economy, the consumer price index in the rest of the world is defined as $P_{t}^{*}=\left[\left(1-\alpha^{*}\right)\left(P_{F, t}^{*}\right)^{1-\eta}+\alpha^{*}\left(P_{H, t}^{*}\right)^{1-\eta}\right]^{1 /(1-\eta)}$. Next recall that we have assumed that $\alpha^{*} \approx 0$ so that $P_{t}^{*}=P_{F, t}^{*}$ and multiplying by $\varepsilon_{t}$, we find $\varepsilon_{t} P_{t}^{*}=\varepsilon_{t} P_{F, t}^{*}$. Using this in (2.60) allows $Y_{t}$ to be written in terms of domestic prices alone. If we then use the definition of the terms of trade, $s_{t}=P_{F, t} / P_{H, t}$, to simplify equation (2.60), the following expression is obtained,

$$
Y_{t}=(1-\alpha)\left(\frac{P_{H, t}}{P_{t}}\right)^{-\eta} C_{t}+\alpha^{*}\left(s_{t} \Psi_{t}\right)^{\eta} C_{t}^{*}
$$

Log-linearizing this equation around the steady state, with $\bar{P}_{H, t}=\bar{P}_{t}, \bar{s}_{t}=1$, and $\bar{\Psi}_{t}=1$ we obtain

\footnotetext{
${ }^{30}$ Comparing equations (2.60) and (2.58) we see that $Y_{t}=Y_{t}(i)\left(P_{H, t}(i) / P_{H, t}\right)^{\theta}$.
} 


$$
\hat{Y}_{t}=(1-\alpha)\left[\eta\left(\hat{P}_{t}-\hat{P}_{H, t}\right)+\hat{C}_{t}\right]+\alpha\left(\eta \hat{s}_{t}+\eta \hat{\Psi}_{t}+\hat{C}_{t}^{*}\right),
$$

where to derive (2.61) used the balanced trade condition that holds in the steady state, so that $\alpha^{*} \bar{C}^{*}=\alpha \bar{C}$, and also the condition that the law of one price holds in the steady state for import goods. Since the consumer price index is defined as $P_{t}=\left[(1-\alpha) P_{H, t}^{1-\eta}+\alpha P_{F, t}^{1-\eta}\right]^{1 /(1-\eta)}$, if log-linearizing it around the steady state with $P_{H, t}=P_{F, t}$ we find

$$
\hat{P}_{t}-\hat{P}_{H, t}=\alpha \hat{s}_{t} .
$$

Plugging (2.62) into (2.61) yields

$$
\hat{Y}_{t}=(1-\alpha) \hat{C}_{t}+\alpha \eta(2-\alpha) \hat{s}_{t}+\alpha \eta \hat{\Psi}_{t}+\alpha \hat{C}_{t}^{*} .
$$

Next, we know that the real exchange rate can be written as

$$
q_{t}=\frac{\varepsilon_{t} P_{t}^{*}}{P_{t}}=\frac{\varepsilon_{t} P_{F, t}^{*}}{P_{t}}=s_{t} \Psi_{t} \frac{P_{H, t}}{P_{t}}
$$

and log-linearizing this about its steady state yields,

$$
\hat{q}_{t}=\hat{s}_{t}+\hat{\Psi}_{t}+\hat{P}_{H, t}-\hat{P}_{t}
$$

Plugging (2.62) into this gives

$$
\hat{q}_{t}=\hat{\Psi}_{t}+(1-\alpha) \hat{s}_{t}
$$

Equation (2.64) shows that there are two reasons for the log deviation of the real exchange rate from its steady state value in which purchasing power parity will hold: the first is incomplete exchange rate pass-through--the log deviation of import goods price from the law of one price, $\hat{\Psi}_{t}$; and the second is the difference in consumption commodity baskets between the home economy and the rest of the world. This deviation is eliminated only when $\alpha=1$, which implies that the small open economy is completely 
open and domestic households consume exactly the same goods as foreign consumers. Rewriting (2.64) in terms of $\hat{s}_{t}$,

$$
\hat{s}_{t}=\frac{1}{1-\alpha}\left(\hat{q}_{t}-\hat{\Psi}_{t}\right)
$$

Substituting (2.65) into (2.63), using $C_{t}^{*} \approx Y_{t}^{*}$, yields

$$
\hat{Y}_{t}=(1-\alpha) \hat{C}_{t}+\frac{\alpha \eta(2-\alpha)}{1-\alpha} \hat{q}_{t}-\frac{\alpha \eta}{1-\alpha} \hat{\Psi}_{t}+\alpha \hat{Y}_{t}^{*}
$$

or rewritten slightly differently,

$$
\hat{C}_{t}=\frac{1}{1-\alpha} \hat{Y}_{t}-\frac{\alpha \eta(2-\alpha)}{(1-\alpha)^{2}} \hat{q}_{t}+\frac{\alpha \eta}{(1-\alpha)^{2}} \hat{\Psi}_{t}-\frac{\alpha}{1-\alpha} \hat{Y}_{t}^{*}
$$

Equation (2.67) shows that the log deviation of consumption from its steady state is positively related to the $\log$ deviation of domestic output and negatively related to the $\log$ deviation of foreign output. In addition, a rise in the $\log$ deviation of the real exchange rate (a depreciation of the home currency) decreases the log deviation of domestic consumption. Similarly a rise in the gap of the law of one price increases the $\log$ deviation of domestic consumption. Note that when $\alpha=0$ (meaning the home economy is a closed economy), the log deviation of consumption from its steady state is completely explained by the log deviation of output.

Recall from (2.25) that the first order condition for consumption intertemporally is $C_{t}^{-\sigma}=\beta R_{t} E_{t}\left(P_{t} / P_{t+1}\right)\left(\chi_{1 t+1} / \chi_{1 t}\right) C_{t+1}^{-\sigma}$. Log-linearizing this around the zero inflation steady state results in $\hat{C}_{t}=E_{t} \hat{C}_{t+1}-(1 / \sigma)\left(\hat{R}_{t}-E_{t} \hat{\Pi}_{t+1}\right)-(1 / \sigma) E_{t} \Delta \hat{\chi}_{1 t+1}$. Then using (2.67) to eliminate $\hat{C}_{t}$ yields the goods market or IS relationship 


$$
\begin{aligned}
& \hat{Y}_{t}=E_{t} \hat{Y}_{t+1}-\frac{1-\alpha}{\sigma}\left(\hat{R}_{t}-E_{t} \hat{\Pi}_{t+1}\right)-\frac{\alpha \eta(2-\alpha)}{1-\alpha} E_{t} \Delta \hat{q}_{t+1} \\
& +\frac{\alpha \eta}{1-\alpha} E_{t} \Delta \hat{\Psi}_{t+1}-\alpha E_{t} \Delta \hat{Y}_{t+1}^{*}-\frac{1-\alpha}{\sigma} E_{t} \Delta \hat{\chi}_{1 t+1}
\end{aligned}
$$

From (2.62) and (2.64) we derive the following expression,

$$
\hat{\Pi}_{t+1}=\hat{\Pi}_{H, t+1}+(\alpha /(1-\alpha)) \Delta \hat{q}_{t+1}-(\alpha /(1-\alpha)) \Delta \hat{\Psi}_{t+1}
$$

and using $(2.69)$ to replace $\hat{\Pi}_{t+1}$ we can rewrite $(2.68)$ in terms of producer prices to obtain

$$
\begin{aligned}
& \hat{Y}_{t}=E_{t} \hat{Y}_{t+1}-\frac{1-\alpha}{\sigma}\left(\hat{R}_{t}-E_{t} \hat{\Pi}_{H, t+1}\right)-\frac{\alpha[\sigma \eta(2-\alpha)+\alpha-1]}{\sigma(1-\alpha)} E_{t} \Delta \hat{q}_{t+1} \\
& +\frac{\alpha(\sigma \eta+\alpha-1)}{\sigma(1-\alpha)} E_{t} \Delta \hat{\Psi}_{t+1}-\alpha E_{t} \Delta \hat{Y}_{t+1}^{*}-\frac{1-\alpha}{\sigma} E_{t} \Delta \hat{\chi}_{1 t+1}
\end{aligned}
$$

Equation (2.70) then states that the log deviation of the domestic output from its steady state depends upon the following six factors. First, it depends positively on the future expected (log) deviation of domestic output. Second, it depends negatively on the deviation of the real interest rate from its steady state value. Third, it depends negatively on the expected change in the log deviation of the real exchange rate from its steady state value. The fourth factor arises from the fact the law of one price does not hold for imported goods in the short run. Thus an expected increase in the gap of the law of one price affects domestic output positively. Fifth, as a small open economy, the demand for domestic output is affected by the world economy. Finally, log deviation of the domestic output can be affected by shocks changing the preference weighting given to future versus present consumption. This effect is positive for contemporaneous temporary shocks to consumption. 


\subsection{The LM Equation}

For convenience, we rewrite $(2.27)$ as $\left(m_{t}\right)^{-\varsigma} R_{t}=C_{t}^{-\sigma} \chi_{1 t}\left(R_{t}-1\right)$, where $m_{t}=M_{t} / P_{t}$. Then evaluating it in steady state yields

$$
(\bar{m})^{-\varsigma} \bar{R}=\bar{C}^{-\sigma} \bar{\chi}(\bar{R}-1)
$$

Log-linearizing (2.27) around this steady-state we obtain

$$
\begin{aligned}
& (\bar{m})^{-\varsigma} \bar{R}\left(1-\sigma \hat{m}_{t}+\hat{R}_{t}\right) \\
& =\bar{C}^{-\sigma} \bar{R} \bar{\chi}_{1}\left(1-\sigma \hat{C}_{t}+\hat{R}_{t}+\hat{\chi}_{1 t}\right)-\bar{C}^{-\sigma} \bar{\chi}_{1}\left(1-\sigma \hat{C}_{t}+\hat{\chi}_{1 t}\right) .
\end{aligned}
$$

Using (2.71) first to eliminate the 1's from the equation and reorganizing common terms yields

$$
\bar{m}^{-\varsigma} \bar{R}\left(-\varsigma \hat{m}_{t}+\hat{R}_{t}\right)=\bar{C}^{-\sigma} \bar{\chi}_{1}(\bar{R}-1)\left(-\sigma \hat{C}_{t}+\hat{\chi}_{1 t}\right)+\bar{C}^{-\sigma} \bar{\chi}_{1} \bar{R} \hat{R}_{t}
$$

Then using (2.71) again in (2.72) to divide out common terms, we obtain

$$
\hat{m}_{t}=(1 / \varsigma)\left[\hat{\sigma}-(1 /(\bar{R}-1)) \hat{R}_{t}-\hat{\chi}_{1 t}\right]
$$

Next using (2.67) to substitute for $\hat{C}_{t}$ in (2.73) gives us

$$
\begin{aligned}
& \hat{m}_{t}=\frac{\sigma}{\varsigma(1-\alpha)} \hat{Y}_{t}-\frac{\beta}{\varsigma(1-\beta)} \hat{R}_{t}-\frac{\alpha \sigma \eta(2-\alpha)}{\varsigma(1-\alpha)^{2}} \hat{q}_{t} \\
& +\frac{\alpha \sigma \eta}{\varsigma(1-\alpha)^{2}} \hat{\Psi}_{t}-\frac{1}{\varsigma} \hat{\chi}_{1 t}-\frac{\alpha \sigma}{\varsigma(1-\alpha)} \hat{Y}_{t}^{*}
\end{aligned}
$$

where $\hat{m}_{t}=\hat{M}_{t}-\hat{P}_{t}$ and where $\bar{i}=(1-\beta) / \beta$ and $\bar{R} \equiv 1+\bar{i}=1 / \beta$. Equation $(2.74)$ then indicates that the log deviation of real money demand from its steady state varies directly with the corresponding deviations in domestic output and the size of the gap in the law of one price and inversely with the corresponding deviations in the domestic interest rate, the real exchange rate, the preference shock to consumption, and foreign output. 


\subsection{The evolution of the Real Exchange Rate and Net Foreign Assets}

The dynamic process describing the movement of the real exchange rate can be derived from "the consumption risk sharing condition"31, obtained by equating the domestic consumption Euler equation in (2.26) with the foreign consumption Euler equation in (2.37) ${ }^{32}$ After simplifying, "the consumption risk-sharing condition" can be written as

$$
E_{t}\left(\frac{C_{t}}{C_{t+1}}\right)^{-\sigma}=\exp \left(-b N F A_{t}+\ln \chi_{2 t}\right) E_{t}\left(\frac{q_{t+1}}{q_{t}}\right)\left(\frac{C_{t}^{*}}{C_{t+1}^{*}}\right)^{-\sigma} .
$$

As discussed earlier, the steady state value of the net foreign asset can be a positive, negative number, or zero depending upon the assumptions about steady state values of the domestic and world interest rate. In our work we have used the assumption that $\beta=\beta^{*}$ to derive $\bar{R}=\bar{R}^{*}$ and $\overline{N F A}=0$. Nevertheless, a steady state value of zero for net foreign assets creates a difficulty for log-linearization. Hence to avoid the problem of dividing by a steady state value of zero, we first totally differentiate (2.75) around the steady state value, ${ }^{33}$ and then rearrange terms to obtain

$$
\hat{C}_{t+1}-\hat{C}_{t}=\frac{1}{\sigma}\left(\hat{q}_{t+1}-\hat{q}_{t}\right)+\hat{C}_{t+1}^{*}-\hat{C}_{t}^{*}-\frac{b}{\sigma} N F A_{t}-\frac{1}{\sigma} \hat{\chi}_{2 t},
$$

where $N F A_{t}$ is treated as a variable so that $d N F A_{t}=N F A_{t}-\overline{N F A}=N F A_{t}$, and the following conditions have been used: $\bar{C}_{t+1} / \bar{C}_{t}=1, \bar{C}_{t+1}^{*} / \bar{C}_{t}^{*}=1, \bar{q}_{t+1} / \bar{q}_{t}=1$,

\footnotetext{
${ }^{31}$ Note that the domestic household's consumption risks are not really shared with the foreign households since the financial markets are assumed to be incomplete.

${ }^{32}$ Alternatively the dynamics of the real exchange rate can be derived from (2.29), the uncovered interest parity condition. Totally differentiating (2.29) around the steady state we find

$\hat{q}_{t}=E_{t} \hat{q}_{t+1}-(1-\alpha)\left(\hat{R}_{t}-E_{t} \hat{\Pi}_{H, t+1}\right)+(1-\alpha)\left(\hat{R}_{t}^{*}-E_{t} \hat{\Pi}_{t+1}^{*}\right)$

$+\alpha\left(\hat{\Psi}_{t}-E_{t} \hat{\Psi}_{t+1}\right)-(1-\alpha) b N F A_{t}+(1-\alpha) \hat{\chi}_{2 t}$

${ }^{33}$ I would like to thank Malin Adolfson who introduced me to this method for solving this problem.
} 
$\bar{\chi}_{1 t+1} / \bar{\chi}_{1 t}=1$, and $\bar{\chi}_{2 t}=1$. Next using (2.67), and $\hat{C}_{t}^{*}=\hat{Y}_{t}^{*}$ in (2.76) to eliminate $\hat{C}_{t}$ and $\hat{C}_{t}^{*}$ yields

$$
\begin{aligned}
& \hat{q}_{t}=E_{t} \hat{q}_{t+1}+\frac{\sigma(1-\alpha)}{\phi}\left(\hat{Y}_{t}-E_{t} \hat{Y}_{t+1}\right)-\frac{\sigma(1-\alpha)}{\phi}\left(\hat{Y}_{t}^{*}-E_{t} \hat{Y}_{t+1}^{*}\right) \\
& +\frac{\alpha \sigma \eta}{\phi}\left(\hat{\Psi}_{t}-E_{t} \hat{\Psi}_{t+1}\right)-\frac{b(1-\alpha)^{2}}{\phi} N F A_{t}+\frac{(1-\alpha)^{2}}{\phi} \hat{\chi}_{2 t}
\end{aligned}
$$

where $\phi=[\alpha(2-\alpha)(\sigma \eta-1)+1]$. Equation (2.77) tells us that the deviation of the real exchange rate from its steady state value increases directly with its expected future value. Secondly, positive current deviations in domestic and foreign output (relative to expected) change the real exchange rate in the opposite direction. Thirdly, import price stickiness also leads the real exchange rate to deviate from its steady state value. Here the stickier is the current price of imports, the larger is the deviation in the real exchange rate. Incomplete asset markets also affect real exchange rates with foreign asset accumulation reducing the deviation in the real exchange rate. Finally, the consumption taste shock and the shock to the risk premium will also produce opposing deviations in the real exchange rate from its steady state value.

Next we derive the equation of motion for net foreign assets. With incomplete pass-through and imperfect asset markets, domestic consumption will rarely equal its production and the current account balance not always equal zero. Nevertheless, a country's current account balance must equal the change in its net holding of foreign assets, that is,

$$
P_{H, t} Y_{t}-P_{t} C_{t}=\frac{\varepsilon_{t} B_{t}^{*}}{R_{t}^{*} \exp \left(-b N F A_{t}+\ln \chi_{2 t}\right)}-\varepsilon_{t} B_{t-1}^{*}
$$

Dividing both sides of (2.78) by $P_{t}$ and using the definition of $N F A_{t}$ gives us 


$$
\frac{P_{H, t}}{P_{t}} Y_{t}-C_{t}=\frac{N F A_{t}}{R_{t}^{*} \exp \left(-b N F A_{t}+\ln \chi_{2 t}\right)}-N F A_{t-1} P_{t-1} / P_{t}
$$

Totally differentiating it about its steady state values (to again avoid the problem of dividing by zero) yields

$$
\frac{d P_{H, t}}{\bar{P}} \bar{Y}-\frac{d P_{t}}{\bar{P}^{2}} \bar{P}_{H} \bar{Y}+\frac{\bar{P}_{H}}{\bar{P}} d Y_{t}-d C_{t}=\frac{d N F A_{t}}{\bar{R}^{*}}-d N F A_{t-1} \bar{P} / \bar{P}
$$

where the zero steady state value of $N F A_{t}$ has been used again. Rewriting it by applying other steady state conditions specifying earlier we obtain

$$
\hat{P}_{H, t}-\hat{P}_{t}+\hat{Y}_{t}-\hat{C}_{t}=N F A_{t} / \bar{R}^{*}-N F A_{t-1} .
$$

From (2.62) and (2.64) we obtain $\hat{P}_{H, t}-\hat{P}_{t}=(\alpha /(1-\alpha)) \hat{\Psi}_{t}-(\alpha /(1-\alpha)) \hat{q}_{t}$. Using this and (2.67) we have

$$
\begin{aligned}
& \beta N F A_{t}-N F A_{t-1}=\frac{\alpha}{(1-\alpha)} \hat{\Psi}_{t}-\frac{\alpha}{(1-\alpha)} \hat{q}_{t}+\hat{Y}_{t} \\
& -\left(\frac{1}{(1-\alpha)} \hat{Y}-\frac{\alpha \eta(2-\alpha)}{(1-\alpha)^{2}} \hat{q}_{t}+\frac{\alpha \eta}{(1-\alpha)^{2}} \hat{\Psi}_{t}-\frac{\alpha}{(1-\alpha)} \hat{Y}_{t}^{*}\right),
\end{aligned}
$$

where $\beta=1 / \bar{R}^{*}$. Collecting common terms and rearranging it yields

$$
\begin{aligned}
& N F A_{t}=\frac{1}{\beta} N F A_{t-1}-\frac{\alpha}{\beta(1-\alpha)} \hat{Y}_{t}+\frac{\alpha[\eta(2-\alpha)+\alpha-1]}{\beta(1-\alpha)^{2}} \hat{q}_{t} \\
& -\frac{\alpha(\eta+\alpha-1)}{\beta(1-\alpha)^{2}} \hat{\Psi}_{t}+\frac{\alpha}{\beta(1-\alpha)} \hat{Y}_{t}^{*}
\end{aligned}
$$

From equation (2.79) we see that the net foreign asset position is positively related to its previous (lagged) value, which implies that the country's net foreign asset position will be characterized by positive persistence. In addition, the log deviation of the domestic output has a negative effect on the net foreign asset position. It initially seemed puzzling. However to understand full effect of output in the equation, we must examine 
the effect of the real exchange rate through equation $(2,77)$. There we find that the $\log$ deviation of the real exchange rate is positively related to the output. Hence when we substitute (2.77) back into (2.79), the combined effect of a positive deviation in domestic output if found to be positive in its overall effect on the net foreign asset position. The same argument is applicable to foreign output but in the opposite direction. As expected, the real exchange rate effect itself is positively related to the net foreign asset position. The reason is that when the real exchange rate goes up, (domestic currency depreciates), imported goods become more expensive relative to the domestic goods and expenditure switching into domestic goods occurs. The law of one price gap, $\hat{\Psi}_{t}$, works in the opposite direction. The bigger that gap, the greater the demand for imported goods and hence the smaller will be the net foreign assets accumulated.

\subsection{Rewriting the Equations of Motion in terms of Efficiency Gaps}

Until now the Phillips's curve equation, the IS, the LM, the real exchange rate, and net foreign asset relationships have all been derived in terms of the log deviation of actual from steady state levels. In this section we transform these measures into perhaps a more familiar forms by rewriting them in terms of the efficiency gaps attributable to the difference between the actual outcomes and the outcomes arising under fully flexible prices and complete asset markets (to be defined more precisely below). We begin by first log-linearizing (2.41) around its steady-state value to obtain

$$
\hat{\varphi}_{H, t}=\hat{W}_{t}-\hat{P}_{t}+\hat{P}_{t}-\hat{P}_{H, t}-\hat{A}_{t} .
$$

Similarly, log-linearizing (2.30) around its steady state values, we obtain 


$$
\hat{W}_{t}-\hat{P}_{t}=\mu \hat{N}_{t}+\sigma \hat{C}_{t}-\hat{\chi}_{1 t}
$$

Plugging (2.81) into (2.80) yields

$$
\hat{\varphi}_{H, t}=\mu \hat{N}_{t}+\sigma \hat{C}_{t}-\hat{\chi}_{1 t}+\hat{P}_{t}-\hat{P}_{H, t}-\hat{A}_{t}
$$

Applying $\hat{P}_{t}-\hat{P}_{H, t}=(\alpha /(1-\alpha)) \hat{q}_{t}-(\alpha /(1-\alpha)) \hat{\Psi}_{t}$, equation $(2.82)$ can be written as

$$
\hat{\varphi}_{H, t}=\mu \hat{N}_{t}+\sigma \hat{C}_{t}-\hat{\chi}_{1 t}+(\alpha /(1-\alpha)) \hat{q}_{t}-(\alpha /(1-\alpha)) \hat{\Psi}_{t}-\hat{A}_{t}
$$

Notice next that $Y_{t}(i)=A_{t} N_{t}(i)$. Dividing each firm's production function by $Y_{t}$, and taking integral with respect to $i$ over the unit interval of firms, we obtain

$$
\int_{0}^{1} \frac{Y_{t}(i)}{Y_{t}} d i=\int_{0}^{1} \frac{A_{t}}{Y_{t}} N_{t}(i) d i=\frac{A_{t}}{Y_{t}} \int_{0}^{1} N_{t}(i) d i
$$

since each firm faces the same productivity shock. Then applying $N_{t}=\int_{0}^{1} N_{t}(i) d i$ and cross multiplying, this expression can be rewritten as

$$
N_{t}=\frac{Y_{t}}{A_{t}} \int_{0}^{1} \frac{Y_{t}(i)}{Y_{t}} d i
$$

Gali and Monacelli (2002) have shown that variations in $\log \int_{0}^{1}\left(Y_{t}(i) / Y_{t}\right) d i$ around the perfect foresight steady state are of second order. Thus up to a first order approximation, this implies the following aggregate relationship

$$
\hat{N}_{t}=\hat{Y}_{t}-\hat{A}_{t}
$$

Substituting (2.84) back into (2.83), together with $(2.67)$, the following expression can be derived for $\hat{\varphi}_{H, t}$, 


$$
\begin{aligned}
& \hat{\varphi}_{H, t}=\mu\left(\hat{Y}_{t}-\hat{A}_{t}\right)-\hat{\chi}_{1 t}+\frac{\alpha}{1-\alpha} \hat{q}_{t}-\frac{\alpha}{1-\alpha} \hat{\Psi}_{t}-\hat{A}_{t} \\
& +\sigma\left(\frac{1}{1-\alpha} \hat{Y}_{t}-\frac{\alpha \eta(2-\alpha)}{(1-\alpha)^{2}} \hat{q}_{t}+\frac{\alpha \eta}{(1-\alpha)^{2}} \hat{\Psi}_{t}-\frac{\alpha}{(1-\alpha)} \hat{Y}_{t}^{*}\right)
\end{aligned}
$$

Collecting common terms and rearranging it we have

$$
\begin{aligned}
& \hat{\varphi}_{H_{s} t}=\frac{\mu+\sigma-\mu \alpha}{1-\alpha} \hat{Y}_{t}-\frac{\phi+\alpha-1}{(1-\alpha)^{2}} \hat{q}_{t} \\
& +\frac{\alpha(\sigma \eta+\alpha-1)}{(1-\alpha)^{2}} \hat{\Psi}_{t}-\frac{\alpha \sigma}{1-\alpha} \hat{Y}_{t}^{*}-(1+\mu) \hat{A}_{t}-\hat{\chi}_{1 t} .
\end{aligned}
$$

Note that when output prices are completely flexible, exchange rate pass-through is complete, the optimal subsidy is paid, and the marginal product of labor is constant each period and equal to $A_{t}$, then $\varphi_{H, t}=W_{t} / P_{H, t} A_{t}=1$ so that real marginal cost is constant and the $\log$ deviation of real marginal cost is zero (i.e., $\hat{\varphi}_{H, t}=0$ ). Hence setting $\hat{\varphi}_{H, t}=0$ in $(2.85)$, the log deviation of output from its steady state value when prices are completely flexible, $\hat{Y}_{t}^{f}$, can be solved for as

$$
\begin{aligned}
& \hat{Y}_{t}^{f}=\frac{\phi+\alpha-1}{(\mu+\sigma-\mu \alpha)(1-\alpha)} \hat{q}_{t}^{f}+\frac{\alpha \sigma}{\mu+\sigma-\mu \alpha} \hat{Y}_{t}^{*} \\
& +\frac{(1+\mu)(1-\alpha)}{\mu+\sigma-\mu \alpha} \hat{A}_{t}+\frac{1-\alpha}{\mu+\sigma-\mu \alpha} \hat{\chi}_{1 t}
\end{aligned}
$$

where $\hat{q}_{t}^{f}$ will be defined more precisely below ${ }^{34}$. First subtracting and then adding $((\mu+\sigma-\mu \alpha) /(1-\alpha)) \hat{Y}_{t}^{f}$ to $(2.85)$ and using $(2.86)$, we obtain

$$
\hat{\varphi}_{H, t}=\frac{\mu+\sigma-\mu \alpha}{1-\alpha} x_{t}-\frac{\phi+\alpha-1}{(1-\alpha)^{2}}\left(\hat{q}_{t}-\hat{q}_{t}^{f}\right)+\frac{\alpha(\sigma \eta+\alpha-1)}{(1-\alpha)^{2}} \hat{\Psi}_{t},
$$

${ }^{34}$ Substituting $\hat{q}_{t}{ }^{f}$ into (2.86) yields $\hat{Y}_{t}^{f}=\frac{\sigma(1-\phi)}{\phi \mu+\sigma} \hat{Y}_{t}^{*}+\frac{\phi(1+\mu)}{\phi \mu+\sigma} \hat{A}_{t}+\frac{\phi}{\phi \mu+\sigma} \hat{\chi}_{1 t}$. 
where $x_{t} \equiv \hat{Y}_{t}-\hat{Y}_{t}^{f}$ is our definition of the output gap and $\left(\hat{q}_{t}-\hat{q}_{t}^{f}\right)$ is our definition of the real exchange rate gap..$^{35}$ Note that when asset markets are complete and import prices exhibit no price stickiness, the deviation of the real marginal cost could be written in terms of the output gap alone (since the real exchange rate gap is proportional to the output gap). Our introduction of sticky import prices and incomplete asset markets then means that deviations in real marginal cost are now attributable to three problems rather than one. Plugging (2.87) back into (2.55), we can then rewrite the Phillips Curve in terms of these gaps as,

$$
\begin{aligned}
& \hat{\Pi}_{H, t}=\frac{\delta}{1+\beta \delta} \hat{\Pi}_{H, t-1}+\frac{\beta}{1+\beta \delta} E_{t} \hat{\Pi}_{H, t+1}+\frac{\gamma(\mu+\sigma-\mu \alpha)}{1-\alpha} x_{t} \\
& -\frac{\gamma(\phi+\alpha-1)}{(1-\alpha)^{2}}\left(\hat{q}_{t}-\hat{q}_{t}^{f}\right)+\frac{\gamma \alpha(\sigma \eta+\alpha-1)}{(1-\alpha)^{2}} \hat{\Psi}_{t} .
\end{aligned}
$$

This then is the Phillips curve for a zero inflation rate steady state written in terms of the output gap, the real exchange rate gap, and exchange rate pass-through--the gap in import prices from the law of one price. This version of the Phillips's curve uses inflation defined in terms of the rate of change of domestic good prices. ${ }^{36}$

Alternatively, the Phillips Curve can be derived in terms of consumer prices. From the definition of the domestic consumer price index, consumer price inflation

${ }^{35}$ Note that we are following Woodford (2003) in defining $x_{t}$ rather than $\hat{Y}_{t}$ as the output gap. Since $\hat{Y}_{t}=\log Y_{t}-\log \bar{Y}$ and $\hat{Y}_{t}^{f}=\log Y_{t}^{f}-\log \bar{Y}, \quad x_{t}=\log Y_{t}-\log Y_{t}^{f}$. This is the distortion in output arising only from the fact that domestic output prices and import good prices are sticky.

${ }^{36}$ Substituting (2.85) into (2.55) yields

$$
\begin{aligned}
& \hat{\Pi}_{H, t}=\frac{\delta}{1+\beta \delta} \hat{\Pi}_{H, t-1}+\frac{\beta}{1+\beta \delta} E_{t} \hat{\Pi}_{H, t+1}+\frac{\gamma(\mu+\sigma-\mu \alpha)}{1-\alpha} \hat{Y}_{t} \\
& -\frac{\gamma(\phi+\alpha-1)}{(1-\alpha)^{2}} \hat{q}_{t}+\frac{\gamma \alpha(\sigma \eta+\alpha-1)}{(1-\alpha)^{2}} \hat{\Psi}_{t}-\frac{\gamma \alpha \sigma}{1-\alpha} \hat{Y}_{t}^{*}-\gamma(1+\mu) \hat{A}_{t}-\gamma \hat{\chi}_{1 t}
\end{aligned}
$$

This is the Phillips curve written in terms of the log deviations of the domestic inflation, output, the real exchange rate, etc. from their steady state values. 
equals $\hat{\Pi}_{t}=(1-\alpha) \hat{\Pi}_{H, t}+\alpha \hat{\Pi}_{F, t}$. Then substituting first (2.57) for $\hat{\Pi}_{F, t}$ then (2.88) for $\hat{\Pi}_{H, t}$ yields

$$
\begin{aligned}
& \hat{\Pi}_{t}=\frac{\delta}{1+\beta \delta} \hat{\Pi}_{t-1}+\frac{\beta}{1+\beta \delta} E_{t} \hat{\Pi}_{t+1}+\gamma(\mu+\sigma-\mu \alpha) x_{t} \\
& -\frac{\gamma(\phi+\alpha-1)}{(1-\alpha)}\left(\hat{q}_{t}-\hat{q}_{t}^{f}\right)+\left(\frac{\gamma \alpha(\sigma \eta+\alpha-1)}{(1-\alpha)}+\frac{\alpha(\theta-1)}{\omega_{M}(1+\beta \delta)}\right) \hat{\Psi}_{t} .
\end{aligned}
$$

Equation (2.89) is then the Phillips curve written in terms of the consumer price index. ${ }^{37}$ Next, we can use $(2.86)$ to rewrite the IS curve in $(2.70)$ in terms of the same gaps as

$$
\begin{aligned}
& x_{t}=E_{t} x_{t+1}-\frac{1-\alpha}{\sigma}\left(\hat{R}_{t}-E_{t} \hat{\Pi}_{H, t+1}\right)+E_{t} \Delta \hat{Y}_{t+1}^{f} \\
& -\frac{\phi+\alpha-1}{\sigma(1-\alpha)} E_{t}\left(\Delta \hat{q}_{t+1}-\Delta \hat{q}_{t+1}{ }^{f}\right)+\frac{\alpha(\sigma \eta+\alpha-1)}{\sigma(1-\alpha)} E_{t} \Delta \hat{\Psi}_{t+1} \\
& -\alpha E_{t} \Delta \hat{Y}_{t+1}^{*}-\frac{1-\alpha}{\sigma} E_{t} \Delta \hat{\chi}_{1 t+1}-\frac{\phi+\alpha-1}{\sigma(1-\alpha)} E_{t} \Delta \hat{q}_{t+1}{ }^{f} .
\end{aligned}
$$

where $\phi+\alpha-1=\alpha[\sigma \eta(2-\alpha)+\alpha-1]$. Then, following Woodford (2003) and others, we can use (2.90) to derive what is called the Wicksellian natural interest rate. Here we define the Wicksellian rate $\hat{r}_{H, t}{ }^{f}$ as the real rate of interest that would arise under perfectly flexible domestic and import price (so that the law of one price holds for import good). Hence from (2.90) we can set $x_{t}=x_{t+1}=0$ and $\Delta \hat{q}_{t+1}=\Delta \hat{q}_{t+1}{ }^{f}$ and $\Delta \hat{\Psi}_{t+1}=0$

$$
\begin{aligned}
& { }^{37} \text { Alternatively we can write the Phillips curve as } \\
& \hat{\Pi}_{t}=\frac{\delta}{1+\beta \delta} \hat{\Pi}_{t-1}+\frac{\beta}{1+\beta \delta} E_{t} \hat{\Pi}_{t+1}+\gamma(\mu+\sigma-\mu \alpha) \hat{Y}_{t}-\frac{\gamma(\phi+\alpha-1)}{1-\alpha} \hat{q}_{t} \\
& +\left[\frac{\gamma \alpha(\sigma \eta+\alpha-1)}{1-\alpha}+\frac{\alpha(\theta-1)}{\omega_{M}(1+\beta \delta)}\right] \hat{\Psi}_{t}-\gamma \alpha \sigma \hat{Y}_{t}^{*}-\gamma(1+\mu)(1-\alpha) \hat{A}_{t}-\gamma(1-\alpha) \hat{\chi}_{1 t}
\end{aligned}
$$


and solve for $\hat{R}_{H, t}-E_{t} \hat{\Pi}_{H, t+1}$ as the log deviation of the Wicksellian natural interest rate from its steady state value. That is,

$$
\hat{r}_{H, t}{ }^{f}=\frac{\sigma}{1-\alpha} E_{t} \Delta \hat{Y}_{t+1}{ }^{f}-\frac{\phi+\alpha-1}{(1-\alpha)^{2}} E_{t} \Delta \hat{q}_{t+1}{ }^{f}-\frac{\alpha \sigma}{1-\alpha} E_{t} \Delta \hat{Y}_{t+1}^{*}-E_{t} \Delta \hat{\chi}_{1 t+1}
$$

Using $\hat{q}_{t}^{f}$ equation first in it we obtain

$$
\hat{r}_{H, t}{ }^{f}=\frac{\sigma}{\phi} E_{t} \Delta \hat{Y}_{t+1}{ }^{f}+\frac{\sigma(\phi-1)}{\phi} E_{t} \Delta \hat{Y}_{t+1}^{*}-E_{t} \Delta \hat{\chi}_{1 t+1} .
$$

and then substituting (2.86) we have

$$
\hat{r}_{H, t}{ }^{f}=\frac{(\phi-1) \alpha \sigma}{\phi \mu+\sigma}\left(\rho_{y}-1\right) \hat{Y}_{t}^{*}-\frac{(1+\mu) \sigma}{\phi \mu+\sigma}\left(1-\rho_{A}\right) \hat{A}_{t}+\frac{\phi \mu}{\phi \mu+\sigma}\left(1-\rho_{1}\right) \hat{X}_{1 t},
$$

where $E_{t} \Delta \hat{Y}_{t+1}{ }^{*}=\left(\rho_{y}-1\right) \hat{Y}_{t}^{*}, E_{t} \Delta \hat{A}_{t+1}=\left(\rho_{A}-1\right) \hat{A}_{t}$, and $E_{t} \Delta \hat{\chi}_{1, t+1}=\left(\rho_{1}-1\right) \hat{\chi}_{1 t}$ have been used. Adding then subtracting $((1-\alpha) / \sigma) \hat{r}_{H, t}{ }^{f}$ from (2.91) into (2.90) we find

$$
\begin{aligned}
& x_{t}=E_{t} x_{t+1}-\frac{1-\alpha}{\sigma}\left(\hat{R}_{t}-E_{t} \hat{\Pi}_{H, t+1}-\hat{r}_{H, t}^{f}\right) \\
& -\frac{\phi+\alpha-1}{\sigma(1-\alpha)} E_{t}\left(\Delta \hat{q}_{t+1}-\Delta \hat{q}_{t+1}{ }^{f}\right)+\frac{\alpha(\sigma \eta+\alpha-1)}{\sigma(1-\alpha)} E_{t} \Delta \hat{\Psi}_{t+1}
\end{aligned}
$$

Here the Wicksellian real rate and the IS equation itself are defined in relation to inflation rates in domestic good prices. As with the Phillips's curve, we can also express the IS equation and corresponding Wicksellian rate in terms of consumer prices. That is, using (2.86) in (2.68) we obtain

$$
\begin{aligned}
& x_{t}=E_{t} x_{t+1}-\frac{1-\alpha}{\sigma}\left(\hat{R}_{t}-E_{t} \hat{\Pi}_{t+1}\right)-\frac{\alpha \eta(2-\alpha)}{1-\alpha} E_{t}\left(\Delta \hat{q}_{t+1}-\Delta \hat{q}_{t+1}^{f}\right) \\
& +\frac{\alpha \eta}{1-\alpha} E_{t} \Delta \hat{\Psi}_{t+1}-\alpha E_{t} \Delta \hat{Y}_{t+1}^{*}-\frac{1-\alpha}{\sigma} E_{t} \Delta \hat{\chi}_{1 t+1}+E_{t} \Delta \hat{Y}_{t+1}^{f}-\frac{\alpha \eta(2-\alpha)}{1-\alpha} E_{t} \Delta \hat{q}_{t+1}{ }^{f}
\end{aligned}
$$

And again using the Wicksellian real rate (defined for consumer prices) we find 


$$
\begin{aligned}
& x_{t}=E_{t} x_{t+1}-\frac{1-\alpha}{\sigma}\left(\hat{R}_{t}-E_{t} \hat{\Pi}_{t+1}-\hat{r}_{t}^{f}\right) \\
& -\frac{\alpha \eta(2-\alpha)}{1-\alpha} E_{t}\left(\Delta \hat{q}_{t+1}-\Delta \hat{q}_{t+1}^{f}\right)+\frac{\alpha \eta}{1-\alpha} E_{t} \Delta \hat{\Psi}_{t+1},
\end{aligned}
$$

where $\hat{r}_{t}^{f}$ is again the log deviation of Wicksellian natural interest rate corresponding to completely flexible domestic output and import prices from the steady state.

$$
\hat{r}_{t}^{f}=\frac{\sigma}{1-\alpha} E_{t} \Delta \hat{Y}_{t+1}^{f}-\frac{\alpha \sigma \eta(2-\alpha)}{(1-\alpha)^{2}} E_{t} \Delta \hat{q}_{t+1}^{f}-\frac{\alpha \sigma}{1-\alpha} E_{t} \Delta \hat{Y}_{t+1}^{*}-E_{t} \Delta \hat{\chi}_{1 t+1}
$$

where $\alpha \sigma \eta(2-\alpha)=\phi-(1-\alpha)^{2}$. Applying $\hat{q}_{t}^{f}$ into it gives

$$
\hat{r}_{t}^{f}=\frac{\sigma(1-\alpha)}{\phi} E_{t} \Delta \hat{Y}_{t+1}{ }^{f}+\frac{\phi \sigma-(1-\alpha) \sigma}{\phi} E_{t} \Delta \hat{Y}_{t+1}^{*}-E_{t} \Delta \hat{\chi}_{1 t+1}
$$

and then using $\hat{Y}_{t}^{f}$ equation we obtain

$$
\begin{aligned}
& \hat{r}_{t}^{f}=\left[\frac{\sigma^{2}(1-\alpha)(1-\phi)}{\phi(\phi \mu+\sigma)}+\frac{\phi \sigma-(1-\alpha) \sigma}{\phi}\right]\left(\rho_{y}-1\right) \hat{Y}_{t}^{*} \\
& -\frac{\sigma(1-\alpha)(1+\mu)}{\phi \mu+\sigma}\left(1-\rho_{A}\right) \hat{A}_{t}+\frac{\phi \mu+\alpha \sigma}{\phi \mu+\sigma}\left(1-\rho_{1}\right) \hat{\chi}_{1 t}
\end{aligned},
$$

To derive the real exchange rate under the perfectly flexible prices and complete asset markets we appeal to the consumption risk sharing condition, that is, equation (2.75).

$$
E_{t}\left(\frac{C_{t}}{C_{t+1}}\right)^{-\sigma}=E_{t}\left(\frac{q_{t+1}}{q_{t}}\right)\left(\frac{C_{t}^{*}}{C_{t+1}^{*}}\right)^{-\sigma} .
$$

Note that when asset markets are complete, the rate of gross return of assets is state contingent, $E_{t} Q_{t, t+1}$, which is equal to $R_{t}$ where, following Gali and Monacelli (2002), the gross risk premium is set to one, that is, $\exp \left(-b N F A_{t}+\ln \chi_{2 t}\right)=1$. Moreover, under 
the assumption of complete asset markets, the uncovered interest parity (UIRP) condition, $R_{t}=R_{t}^{*} \varepsilon_{t} / \varepsilon_{t+1}$, must hold. Solving (2.95) recursively for $C_{t}$ yields,

$$
C_{t}^{\sigma}=q_{t}\left(C_{t}^{*}\right)^{\sigma} E_{t}\left(\frac{C_{t+1}{ }^{\sigma}}{q_{t+1}\left(C_{t+1}{ }^{*}\right)^{\sigma}}\right),
$$

where $k \subseteq[0, \infty]$. Under the assumption of complete asset markets, both domestic and foreign households consumption risks can be completely insured through contingent claims. Without loss of generality, then, we can assume that $E_{t}\left(C_{t+k}{ }^{\sigma} / q_{t+k}\left(C_{t+k}{ }^{*}\right)^{\sigma}\right)=\alpha^{*} / \alpha$. Using it in (2.96) and then log-linearizing gives

$$
\sigma \hat{C}_{t}=\hat{q}_{t}+\sigma \hat{C}_{t}^{*}
$$

Plugging the goods market clearing condition (2.67) into (2.97) we obtain

$$
\frac{\sigma}{1-\alpha} \hat{Y}_{t}-\frac{\alpha \sigma \eta(2-\alpha)}{(1-\alpha)^{2}} \hat{q}_{t}-\frac{\alpha \sigma}{1-\alpha} \hat{Y}_{t}^{*}=\hat{q}_{t}+\sigma \hat{Y}_{t}^{*}
$$

where conditions, $\hat{\Psi}_{t}=0$, and $\hat{C}_{t}^{*}=\hat{Y}_{t}^{*}$ have been used. Rearranging it yields

$$
\hat{q}_{t}^{f}=\frac{\sigma(1-\alpha)}{\phi}\left(\hat{Y}_{t}^{f}-\hat{Y}_{t}^{*}\right)
$$

Substituting $\hat{Y}_{t}^{f}$ equation into it gives

$$
\hat{q}_{t}^{f}=\frac{\sigma(\alpha-1)(\sigma+\mu)}{\phi \mu+\sigma} \hat{Y}_{t}^{*}+\frac{\sigma(1-\alpha)(1+\mu)}{\phi \mu+\sigma} \hat{A}_{t}+\frac{\sigma(1-\alpha)}{\phi \mu+\sigma} \hat{\chi}_{1 t},
$$

where $\hat{q}_{t}^{f}$ denotes the real exchange rate under completely flexible prices and complete asset markets. Then the real exchange rate equation (2.77) can be rewritten in term of its efficiency gap as 


$$
\begin{aligned}
& \hat{q}_{t}-\hat{q}_{t}^{f}=E_{t}\left(\hat{q}_{t+1}-\hat{q}_{t+1}^{f}\right)+\frac{\sigma(1-\alpha)}{\phi}\left(x_{t}-E_{t} x_{t+1}\right) \\
& +\frac{\alpha \sigma \eta}{\phi}\left(\hat{\Psi}_{t}-E_{t} \hat{\Psi}_{t+1}\right)-\frac{b(1-\alpha)^{2}}{\phi} N F A_{t}+\frac{(1-\alpha)^{2}}{\phi} \hat{\chi}_{2 t}
\end{aligned}
$$

Next, we can rewrite the LM equation (2.74) in terms of these gaps. Note that from our analytical framework, the real balance equation determines real balances recursively given the equilibrium values of output, the interest rate, the real exchange rate, the law of one price gap, the foreign output, and the demand shock. It follows that the $\log$ deviation of the real balances associated with the flexible prices and complete asset markets from its steady state value can be defined as

$$
\begin{aligned}
& \hat{m}_{H, t}{ }^{f}=\frac{\sigma}{\varsigma(1-\alpha)} \hat{Y}_{t}^{f}-\frac{\beta}{\varsigma(1-\beta)}\left(\hat{r}_{H, t}^{f}+\hat{\Pi}_{H, t}{ }^{f}\right) \\
& -\frac{\alpha \sigma \eta(2-\alpha)}{\varsigma(1-\alpha)^{2}} \hat{q}_{t}^{f}-\frac{\alpha \sigma}{\varsigma(1-\alpha)} \hat{Y}_{t}^{*}-\frac{1}{\varsigma} \hat{\chi}_{1 t}
\end{aligned}
$$

Note that $\hat{R}_{H, t}=\hat{r}_{t}+\hat{\Pi}_{H, t}$, where $\hat{r}_{t}$ denotes the real interest rate. Subtracting $(2.100)$ from (2.74) yields

$$
\begin{aligned}
& \hat{m}_{t}-\hat{m}_{H, t}{ }^{f}=\frac{\sigma}{\varsigma(1-\alpha)} x_{t}-\frac{\beta}{\varsigma(1-\beta)}\left(\hat{R}_{t}-\hat{r}_{H, t}{ }^{f}-\hat{\Pi}_{H, t}{ }^{f}\right) \\
& -\frac{\alpha \sigma \eta(2-\alpha)}{\varsigma(1-\alpha)^{2}}\left(\hat{q}_{t}-\hat{q}_{t}^{f}\right)+\frac{\alpha \sigma \eta}{\varsigma(1-\alpha)^{2}} \hat{\Psi}_{t}
\end{aligned} .
$$

Note that $\hat{m}_{H, t}{ }^{f}$ is defined in terms of $\hat{r}_{H, t}{ }^{f}$ and $\hat{\Pi}_{H, t}{ }^{f}$. When the model is expressed in terms of consumer prices, $\hat{\Pi}_{t}$, then we need to modify $(2.100)$ to be

$$
\begin{aligned}
& \hat{m}_{t}^{f}=\frac{\sigma}{\varsigma(1-\alpha)} \hat{Y}_{t}^{f}-\frac{\beta}{\varsigma(1-\beta)}\left(\hat{r}_{t}^{f}+\hat{\Pi}_{t}^{f}\right) \\
& -\frac{\alpha \sigma \eta(2-\alpha)}{\varsigma(1-\alpha)^{2}} \hat{q}_{t}^{f}-\frac{\alpha \sigma}{\varsigma(1-\alpha)} \hat{Y}_{t}^{*}-\frac{1}{\varsigma} \hat{\chi}_{1 t}
\end{aligned}
$$

Correspondingly (2.101) is modified to be 


$$
\begin{aligned}
& \hat{m}_{t}-\hat{m}_{t}^{f}=\frac{\sigma}{\zeta(1-\alpha)} x_{t}-\frac{\beta}{\zeta(1-\beta)}\left(\hat{R}_{t}-\hat{r}_{t}^{f}-\hat{\Pi}_{t}^{f}\right) \\
& -\frac{\alpha \sigma \eta(2-\alpha)}{\zeta(1-\alpha)^{2}}\left(\hat{q}_{t}-\hat{q}_{t}^{f}\right)+\frac{\alpha \sigma \eta}{\zeta(1-\alpha)^{2}} \hat{\Psi}_{t}
\end{aligned}
$$

Finally, the net foreign asset position equation (2.79) can be rewritten in terms of the output gap as

$$
\begin{aligned}
& N F A_{t}=\frac{1}{\beta} N F A_{t-1}-\frac{\alpha}{\beta(1-\alpha)} x_{t}+\frac{\alpha[\eta(2-\alpha)+\alpha-1]}{\beta(1-\alpha)^{2}} \hat{q}_{t} \\
& -\frac{\alpha(\eta+\alpha-1)}{\beta(1-\alpha)^{2}} \hat{\Psi}_{t}-\frac{\alpha}{\beta(1-\alpha)} \hat{Y}_{t}^{f}+\frac{\alpha}{\beta(1-\alpha)} \hat{Y}_{t}^{*}
\end{aligned}
$$

Equation (2.104) is written in terms of the output gap. However, the assumption of under complete asset markets and flexible prices, the current account in equation (2.78) is always balanced (zero) and assets are neither accumulated nor decumulated. ${ }^{38}$ That is, since under complete asset markets, contingent claims are realized as a function of the shocks hitting the system without a change in net wealth,

$$
N F A_{t}^{f}=0
$$

Hence (2.104) can also be used to describe the log deviation of net foreign assets from its flexible price, complete asset market value.

Equations (2.88) (or (2.89)), (2.92) (or (2.93)), (2.99), (2.103), and (2.104) are then the five basic expressions needed to describe the dynamics of our small open

${ }^{38} N F A_{t}^{f}=0$ does not mean that net exports, $(N X)_{t}^{f}$ are zero (only that the current account is balanced each period). Since $N X_{t}=P_{H, t} Y_{t}-P_{t} C_{t}$, we can solve for the time path $\hat{N} \hat{X}_{t}{ }^{f}=\left(\alpha[\eta(2-\alpha)+\alpha-1] / \beta(1-\alpha)^{2}\right) \hat{q}_{t}{ }^{f}-\alpha / \beta(1-\alpha)\left(\hat{Y}_{t}{ }^{f}-\hat{Y}_{t}{ }^{*}\right)$ which will equal the flow realization of period specific contingent claims arising under complete asset markets. 
economy. ${ }^{39}$ They represent five of the six pillars needed to represent both the equilibrium conditions of a well-specified general equilibrium system and the movements of the system's endogenous variables about that equilibrium. Implicitly there are eight variables contained in these five equations: the output gap, the consumer price index inflation, the nominal interest rate, the money supply, the real exchange rate, the net foreign asset position, the law of one price gap, and the foreign output. To close the model, three more equations are needed. One of these will be the monetary policy equation that specifies either an interest rate policy or a money supply process. Before introducing this, however, we first complete the remaining parts of the model by developing the exchange rate pass-through equation or the law of one price gap and then defining the process that describes the evolution of foreign output.

Beginning with exchange rate pass-through, we know from our earlier discussion of sticky import prices that $\hat{\Psi}_{t}=\hat{\varepsilon}_{t}+\hat{P}_{t}^{*}-\hat{P}_{F, t}$. Then using the definition of the real exchange rate, ${ }^{40} \hat{\varepsilon}_{t}=\hat{q}_{t}+\hat{P}_{t}-\hat{P}_{t}^{*}$, to eliminate $\hat{\varepsilon}_{t}$ in this equation yields $\hat{\Psi}_{t}=\hat{q}_{t}+\hat{P}_{t}-\hat{P}_{F, t}$. Hence rewriting the exchange rate pass-through relation in terms of inflation rates, we find

$$
\hat{\Psi}_{t}=\hat{\Psi}_{t-1}+\hat{q}_{t}-\hat{q}_{t-1}+\hat{\Pi}_{t}-\hat{\Pi}_{F, t}
$$

\footnotetext{
${ }^{39}$ With monetary policy specified in terms of an interest rate rule and an inflation target of zero, money supply is determined endogenously by the LM curve (equation) and the price level implicit in the inflation target.

${ }^{40}$ The depreciation of currency is written as $\Delta \hat{\varepsilon}_{t}=\hat{q}_{t}-\hat{q}_{t-1}+(1-\alpha) \hat{\Pi}_{H, t}-(1-\alpha) \hat{\Pi}_{F, t}$, or $\Delta \hat{\varepsilon}_{t}=\hat{q}_{t}-\hat{q}_{t-1}+\hat{\Pi}_{t}-(1-\alpha) \hat{\Pi}_{t}^{*}$.
} 
Next using equation (2.69), $\hat{\Pi}_{t}=\hat{\Pi}_{H, t}+(\alpha /(1-\alpha)) \Delta \hat{q}_{t}-(\alpha /(1-\alpha)) \Delta \hat{\Psi}_{t}$, in (2.106) to eliminate $\hat{\Pi}_{t}$ gives

$$
\hat{\Psi}_{t}=\hat{\Psi}_{t-1}+(1-\alpha) \hat{\Pi}_{H, t}-(1-\alpha) \hat{\Pi}_{F, t}+\hat{q}_{t}-\hat{q}_{t-1} .
$$

Lastly, in a small open economy, foreign output is exogenous. Here we specify it in the form of a stochastic shock facing the small open economy. In particular, foreign output is assumed to follow an AR (1) process,

$$
\ln Y_{t}^{*}=\rho_{y} \ln Y_{t-1}^{*}+\varepsilon_{y, t}
$$

where $\varepsilon_{y}$ is a white nose process with a zero mean and a constant standard error $\sigma_{y}$. With the exchange rate pass through equation derived in terms of deviations from the law of one price and foreign output defined we have seven of our eight relationships. We now close the model by defining a general form for the monetary policy rule.

\subsection{The Interest Rate Reaction Function}

In the money literature, economists have used either the supply of money or the rate of interest to target the assumed zero inflation steady state of the model. Either way closes the model by placing the missing exogenous instrumental variable under the control of the monetary authority. Recently, however, there has been an increasing trend towards the adoption of the short-term interest rate as the relevant central bank control variable. Control of the time path of a short-term nominal interest rate, it is argued, reflects more closely the actual conduct of monetary policy as practiced by central banks such as the U.S. Federal Reserve, the European Central Bank, and the Bank of Canada. We continue in this tradition by assuming that in our model the monetary authority 
explicitly chooses the nominal interest rate as its policy instrument. This implies the supply of money will be determined endogenously through the LM equation.

As Woodford (2003) has argued, the central issue facing monetary policy theory is to provide principles that can be used to select a desirable rule for a central bank when setting the interest rate as its operating target. One early example of such an optimal monetary policy rule set in terms of an interest rate is due to Wicksell $(1898,1907)$. Woodford argues (2003, p. 38) that Wicksell's proposal can be represented mathematically as a commitment by the central bank to set its operating target $i_{t}$ as $i_{t}=\bar{i}+\phi_{p} p_{t}$, where $\bar{i}$ is the nominal interest rate, $p_{t}$ is the log of some general price index (the one that policy aims to stabilize) and $\phi_{p}$ is a positive response coefficient, and is greater than one. ${ }^{41}$

Taylor (1993) provides a more contemporary example. According to what has become the Taylor rule, the interest rate should be set equal to a constant (corresponding to the steady state real rate plus the targeted rate of inflation) plus a linear function of the gap between current and targeted inflation and the gap between actual and potential output. That is, $i_{t}=\bar{i}+\phi_{\pi}\left(\pi_{t}-\pi^{t \text { arget }}\right)+\phi_{y}\left(y_{t}-y_{t}{ }^{p}\right)$, where $\pi_{t}$ is the rate of inflation (the change in the log GDP deflator over the previous four quarters), $\pi^{\text {targ } e t}$ is the targeted rate of inflation, $y_{t}$ is $\log$ of actual output, and $y_{t}{ }^{p}$ is $\log$ "potential" output. Similar to $\phi_{p}$, $\phi_{\pi}$ and $\phi_{y}$ are two response coefficients (with $\phi_{\pi}=\phi_{y}=0.5$, see Taylor, 1993), Following Taylor, researchers (see Taylor, 1999) have proposed many variants of this monetary

\footnotetext{
${ }^{41}$ For a more detailed discussion, see Woodford (2003), chapter 1.
} 
policy rule, including adding such things as interest-rate smoothing and using forecasted rather than contemporary values.

In our small open economy model, inefficiency arises and welfare falls because contemporary shocks are not incorporated into the price setting behavior adopted by monopolistically competitive domestic producers and foreign good importers in the presence of incomplete asset markets. Hence because of incomplete asst markets and sticky prices, actual outcomes differ from the efficient levels that would arise under flexible prices. Monetary policy is then designed to use the available information on these gaps to set the interest rate to counter the effects of sticky prices. For this reason, monetary policy can be described as setting an interest rate as a function of the gaps arising between the interest rate, level of output, inflation rate, the real exchange rate, and the law of one price gap and the levels these values that would take under complete asset markets and perfectly flexible prices.

From this perspective, then, we can describe a general monetary policy rule that could be applied to a number of different information and policy objective cases. One such example would be:

$$
i_{t}=\left(1-\phi_{r}\right)\left[\begin{array}{l}
i_{t}^{\text {targ } e t}+\phi_{\pi}\left(\pi_{t+j}-\pi_{t}^{t \text { arg } e t}\right)+\phi_{x}\left(y_{t+j}-y_{t}^{t \text { arg } e t}\right) \\
+\phi_{q}\left(q_{t+j}-q_{t}^{t a r g e t}\right)+\phi_{\Psi}\left(\Psi_{t+j}-\Psi_{t}^{t \text { arget }}\right)
\end{array}\right]+\phi_{r} i_{t-k}
$$

where the efficiency gaps targeted by monetary policy can be defined either in terms of actual, expected, contemporary or forecasted variables (i.e., $\mathrm{j} \geq 0$ ). Following Clarida, Gali, and Gertler $(1997,1998)$, we also include an interest rate smoothing term $(k \geq 0)$ to allow the policy rule to account for the degree of persistence often found in the data and also present in our model (due to indexation and the particular form of the adjustment 
cost in import prices). The formulation also allows recognition that the specific gaps being targeted will depend upon the information available to the central bank as well as on the objectives ascribed to policy makers.

Finally, because our model is derived in terms of variables reflecting the log deviation from the steady state, it is convenient to rewrite the general policy rule in terms of these deviations. Adopting the use of gross interest rates, (2.109) becomes

$$
\hat{R}_{t}=\left(1-\phi_{r}\right)\left[\begin{array}{l}
\left(\hat{r}_{H, t}^{\arg e t}+\hat{\Pi}_{H, t+j}^{e}\right)+\phi_{\pi}\left(\hat{\Pi}_{H, t+j}-\hat{\Pi}_{H, t}^{t \arg e t}\right)+\phi_{x}\left(\hat{Y}_{t+j}-\hat{Y}_{t}^{t \operatorname{targ} e t}\right) \\
+\phi_{q}\left(\hat{q}_{t+j}-\hat{q}_{t}^{t \text { arg } e t}\right)+\phi_{\Psi}\left(\hat{\Psi}_{t+j}-\hat{\Psi}_{t}^{t \operatorname{targ} e t}\right)
\end{array}\right]+\phi_{r} \hat{R}_{t-k} \cdot(
$$

When the policy rule is expressed in terms of consumer rather than producer prices, (2.110) is modified to become

$$
\hat{R}_{t}=\left(1-\phi_{r}\right)\left[\begin{array}{l}
\left(\hat{r}_{t}^{t \arg e t}+\hat{\Pi}_{t+j}^{e}\right)+\phi_{\pi}\left(\hat{\Pi}_{t+j}-\hat{\Pi}_{t}^{t \arg e t}\right)+\phi_{x}\left(\hat{Y}_{t+j}-\hat{Y}_{t}^{t \operatorname{targ} e t}\right) \\
+\phi_{q}\left(\hat{q}_{t+j}-\hat{q}_{t}^{t \arg e t}\right)+\phi_{\Psi}\left(\hat{\Psi}_{t+j}-\hat{\Psi}_{t}^{t \operatorname{targ} e t}\right)
\end{array}\right]+\phi_{r} \hat{R}_{t-k} .
$$

In this model, the central bank exists to maximize the utility of its households. This implies that in the presence of shocks and Calvo pricing, the central bank will use its ability to set interest rates to alter aggregate demand whenever sticky prices lead actual output to diverge from its flexible price value and so close the welfare reducing output gap. Hence the question arises, what does the central bank do when prices are fully flexible and intervention is not required? The question of particular interest is what will be the value of actual inflation each period when prices are fully flexible and asset markets are complete? More particularly, what meaning should we attach to $\hat{\Pi}_{H, t}^{f}$ or $\hat{\Pi}_{t}{ }^{f}$ in this analysis? 
Had we assumed a monetary policy rule that treated the money supply as exogenous, money prices would typically vary each period in response to the model's shocks and hence the actual inflation rate would be expected to change from period to period about its steady state value of zero (as output responds to maintain its period specific optimal value). This is because the different shocks would typically change both the equilibrium real rate of interest and level of aggregate output. Then because the demand for money is a function of both output and the nominal (and real) interest rate, the real demand for money will change across equilibria. With the nominal supply of money fixed, money prices would need to change to allow the real stock of money to adjust and restore equilibrium in the money market.

However, the central bank is not modeled as controlling the nominal supply of money in our analysis. Rather we follow the current literature in assuming that the central bank will adopt an interest rate rule and let the supply of money adjust endogenously. Nevertheless it is important to see that if the central bank continues to set the interest rate to maintain its commitment to the steady state zero rate of inflation, a particular strategy must be followed to achieve this zero inflation rate outcome. That is, even though when prices are free to vary and relative price flexibility is itself sufficient to keep the economy at its full employment level each period without central bank intervention, the central bank needs to preserve its targeted zero rate of inflation by 'following the market' in setting its money and real rate of interest and accommodating all changes in the demand for nominal money. The question that remains is if it does so, will the aggregate price level stay fixed or will it vary each period? 
It turns out that to maintain a steady state rate of inflation at zero, the central bank has two choices; it can target a zero rate of inflation each and every period, or it can target a constant price level. The former would be achieved by 'following the market " and setting the Wicksellian natural rate of interest in each period. The implication of following this approach, however, is that random period shocks will produce period by period changes in the price level that result in the measured rate of inflation departing from zero each period. Under inflation targeting, these period specific departures are not corrected in pursuing the zero inflation rate target in the following period. However, because the random shocks have zero mean, the law of large numbers implies that a zero inflation targeting strategy over the long run would preserve the average rate of inflation at zero and a constant price level over the long run. ${ }^{42}$

Alternatively, the central bank could target a constant price level. This strategy differs from the previous discussion of targeting a zero inflation rate in that the random period specific departures of the actual or measured rate of inflation from zero are reversed rather than ignored. For our purposes, the key difference is that central bank's interest rate would need to depart from the Wicksellian natural rate each period to compensate for the random departures from the fixed price target. Under full information, or to the extent that current shocks to the price level could be recognized or anticipated, the Wicksellian rate would deliberately depart from the Wicksellian rate to preserve price level constancy this period to achieve the goal of $\hat{\Pi}_{H, t}{ }^{f}$ or $\hat{\Pi}_{t}^{f}=0$ each

\footnotetext{
42 Another way of putting this is that setting the Wicksellian rate each period does preserve a zero rate of inflation each period but also produces a period-specific transitory effect on the price level needed to respond to the period-specific change in the Wicksellian natural rate of interest. Then because this transitory effect is response to a "real" period-specific effect, it should not be countered by policy. Then because all random real shocks to the price level have zero mean, the period-specific shocks will be strictly transitory and offset each other over the long run to preserve price level constancy in the long run.
} 
period. Only under flexible prices and with full information would we expect that the price level could be held constant and hence the flexible price zero inflation rate achieved.

In the work that follows, we take the former route and assume that the central bank adopts zero inflation rate targeting. This is interpreted as meaning that with sufficient information the central bank would target the Wicksellian rate each period and so tolerate period specific variations in the price level and actual rate of inflation when pursuing its zero inflation rate target. The question that remains what will $\hat{\Pi}_{H, t}{ }^{f}$ or $\hat{\Pi}_{t}^{f}$ be in such circumstances?

As discussed earlier (see section 2.3), with perfectly flexible prices and monopolistic competition in the small open economy, the representative firm, $i$, will set its price using the standard markup formula,

$$
P_{H, t}^{f}(i)=\frac{\theta}{\theta-1} M C_{t}(i)
$$

where $P_{H, t}{ }^{f}(i)$ is the nominal price set by firm $i, \theta /(\theta-1)$ is the markup, and $M C_{t}(i)$ is the firm's nominal marginal cost. When the government uses an output subsidy to completely offset firm's market power (as it does in our model), the firm will set its price equal to its nominal marginal cost. In a symmetric equilibrium, with economy wide competitive factor markets, $(2.112)$ becomes

$$
P_{H, t}^{f}=\frac{\theta}{(\theta-1)(1+\tau)} M C_{t}=M C_{t},
$$

where $\theta /(\theta-1)(1+\tau)=1$. Lagging (2.113) one period and dividing it back into (2.113) and then log-linearizing the result, we obtain 


$$
\hat{\Pi}_{H, t}^{f}=M \hat{C}_{t}-M \hat{C}_{t-1}=\Delta M \hat{C}_{t}
$$

Equation (2.114) indicates that the inflation rate that would prevail in the absence of price stickiness will equal the rate of change in its nominal marginal cost. Then using (2.41) in (2.114) we can begin to attribute the departures in the actual inflation rate from zero to the shocks underlying shocks the changes in marginal cost, that is,

$$
\begin{aligned}
& \hat{\Pi}_{H, t} f=\left(\hat{W}_{t}-\hat{P}_{t}+\hat{P}_{t}-\hat{A}_{t}\right)-\left(\hat{W}_{t-1}-\hat{P}_{t-1}+\hat{P}_{t-1}-\hat{A}_{t-1}\right) \\
& =\left(\hat{W}_{t}-\hat{P}_{t}\right)-\left(\hat{W}_{t-1}-\hat{P}_{t-1}\right)+\hat{\Pi}_{t}^{f}-\Delta \hat{A}_{t} .
\end{aligned}
$$

Substituting (2.81) first in (2.115), and then using (2.84) and (2.67) yields

$$
\begin{aligned}
& \hat{\Pi}_{H, t}{ }^{f}=\hat{\Pi}_{t}{ }^{f}+\left[\frac{\mu+\sigma-\mu \alpha}{1-\alpha} \hat{Y}_{t}^{f}-\frac{\alpha \sigma \eta(2-\alpha)}{(1-\alpha)^{2}} \hat{q}_{t}{ }^{f}-\mu \hat{A}_{t}-\hat{\chi}_{1 t}-\frac{\alpha \sigma}{(1-\alpha)} \hat{Y}_{t}^{*}\right] \\
& -\left[\frac{\mu+\sigma-\mu \sigma}{1-\alpha} \hat{Y}_{t-1}{ }^{f}-\frac{\alpha \sigma \eta(2-\alpha)}{(1-\alpha)^{2}} \hat{q}_{t-1}{ }^{f}-\mu \hat{A}_{t-1}-\hat{\chi}_{1 t-1}-\frac{\alpha \sigma}{(1-\alpha)} \hat{Y}_{t-1}^{*}\right]-\Delta \hat{A}_{t}
\end{aligned}
$$

Next we can write $\hat{\Pi}_{t}^{f}$ in terms of $\hat{\Pi}_{H, t}{ }^{f}$ by using the fact that $\hat{\Pi}_{t}=(1-\alpha) \hat{\Pi}_{H, t}+\alpha \hat{\Pi}_{F, t}$, to initially derive its flexible price version as

$$
\hat{\Pi}_{t}^{f}=(1-\alpha) \hat{\Pi}_{H, t}^{f}+\alpha \hat{\Pi}_{F, t}^{f}
$$

Then using the result that under complete asset markets and completely flexible import good prices, the law of one price must hold for import goods so that $\hat{\Pi}_{F, t}^{f}=0$.

Using this result first in (2.117), and then substituting that result into (2.116) yields

$$
\hat{\Pi}_{H_{t} t}^{f}=\frac{1}{\alpha}\left[\begin{array}{c}
\frac{\mu+\sigma-\mu \alpha}{1-\alpha}\left(\hat{Y}_{t}^{f}-\hat{Y}_{t-1}^{f}\right)-\frac{\alpha \sigma \eta(2-\alpha)}{(1-\alpha)^{2}}\left(\hat{q}_{t}^{f}-\hat{q}_{t-1}{ }^{f}\right) \\
-(1+\mu)\left(\hat{A}_{t}-\hat{A}_{t-1}\right)-\left(\hat{\chi}_{1 t}-\hat{\chi}_{1 t-1}\right)-\frac{\alpha \sigma}{(1-\alpha)}\left(\hat{Y}_{t}^{*}-\hat{Y}_{t-1}^{*}\right)
\end{array}\right]
$$


Note that since $\hat{\Pi}_{H, t}{ }^{f}$ is a function only of the differences in flexible price deviations from the steady-state and or differences in exogenous shocks directly, the evolution of inflation is dependent only upon the period specific shocks impacting the model with its expected value (and mean value through time) equaling zero.

Alternatively we could have used (2.117) to solve for the rate of inflation under flexible prices in terms of consumer prices, $\hat{\Pi}_{t}{ }^{f}$, rather than domestic prices and so derive,

$$
\hat{\Pi}_{t}^{f}=\frac{1-\alpha}{\alpha}\left[\begin{array}{c}
\frac{\mu+\sigma-\mu \alpha}{1-\alpha}\left(\hat{Y}_{t}^{f}-\hat{Y}_{t-1}^{f}\right)-\frac{\alpha \sigma \eta(2-\alpha)}{(1-\alpha)^{2}}\left(\hat{q}_{t}^{f}-\hat{q}_{t-1}^{f}\right) \\
-(1+\mu)\left(\hat{A}_{t}-\hat{A}_{t-1}\right)-\left(\hat{\chi}_{1 t}-\hat{\chi}_{1 t-1}\right)-\frac{\alpha \sigma}{(1-\alpha)}\left(\hat{Y}_{t}^{*}-\hat{Y}_{t-1}^{*}\right)
\end{array}\right]
$$

With this final modification, all of the equations required to describe the small open economy have been derived. To solve the model in terms of a given initial domestic output price level, we need to use the following eight equations: $(2.57),(2.88),(2.92)$, (2.101), (2.104), (2.107), and (2.110). To derive the model based on a given consumer price level, we need to use (2.57), (2.89), (2.93), (2.99), (2.103), (2.104), (2.106), and (2.111). In addition, we need equations (2.3), (2.22), (2.39), and (2.108) which describe the exogenous shocks that impact the model. 


\section{Chapter 3}

\section{The Loss Function for a Small Open Economy}

Most often in the current money literature, the central bank is assumed to operate to minimize the expected value of a loss function that is quadratic in two terms: the inflation rate and the output gap. Often this is given the form, $L_{t}=\hat{\Pi}_{t}{ }^{2}+\lambda x_{t}{ }^{2}$, where the parameter $\lambda \geq 0$ denotes the relative weight that the central bank places on output stabilization. ${ }^{43}$ While such a specification is plausible, it is ultimately ad hoc, especially when the value of $\lambda$ is assigned arbitrarily. More fundamentally, when the policy maker's objective is to improve the efficiency of the economic system for the representative household, the loss function measuring the cost of avoidable market inefficiencies should be derived from the representative household's utility function. Here Rotemberg and Woodford (1999) and Woodford (2003) have done pioneering research and have shown that by taking a second-order Taylor series expansion of the representative household's utility function, it is possible to derive a loss function that has exactly the same form as that commonly used in the literature except that the value of $\lambda$ is now defined in terms of the structural parameters of the model rather than simply assigned.

\footnotetext{
${ }^{43}$ The central bank's objective function can be alternatively specified to maximize the expected value of $U_{t}=\lambda x_{t}-\hat{\Pi}_{t}{ }^{2}$ (see Barro and Gordon, 1983) or to minimize $L_{t}=\hat{\Pi}_{t}{ }^{2}+\lambda_{1} x_{t}{ }^{2}+\lambda_{2}\left(i_{t}-i_{t-1}\right)^{2}$ where $i_{t}$ is the short-term interest rate (see Rudebusch and Svensson, 1999).
} 
Rotemberg and Woodford's loss function has also been extended to the open economy where relative prices, such as the nominal exchange rate, the terms of trade, and the real exchange rate, become important new endogenous variables. It is then of interest to know whether these variables and/or their departure from their flexible price alternative should be of concern to policy makers. Gali and Monacelli (2002) show that the policy maker's objective function in an open economy is isomorphic to the closed economy, a condition that also implies that the flexible exchange rates are the desirable policy alternative. A similar conclusion is obtained by Clarida et al $(2001,2002)$. It is important to note, however, that the conclusion that the small open economy's loss function need not explicitly include the exchange rate is derived only after invoking a number of special assumptions. For example, Gali and Monacelli assume specifically that the intertemporal elasticity of substitution in consumption, $1 / \sigma$, is one (that is, takes log utility form); the elasticity of substitution between domestic and foreign goods, $\eta$, is also equal to one; and the household is assumed to have access to a complete set of contingent asset markets. However, Kirsanova et al (2006) show that by explicitly introducing shocks into the international risk sharing condition makes the small open economy's loss function no longer isomorphic to the closed economy and introduces the terms of trade as a new term in the loss function. Similarly, De Paoli (2006) assumes that there is a home bias in consumption and, as in Sutherland (2002), finds that including the exchange rate as part of the stabilization goals of monetary policy can be welfare improving from the small open economy point of view.

Note that in the early literature cited above two assumptions were commonly imposed: financial markets were assumed to be complete and the degree of exchange rate 
pass-through was assumed to be one (see Gali and Monacelli, 2002, Clarida et al, 2001). These two assumptions greatly simplify the analysis. Hence it can be shown that under the assumption of complete asset markets, consumption gaps and terms of trade gaps (or the real exchange rate gaps) move proportionately with the output gap, and so the output gap can replace the terms of trade gaps in the policy maker's objective function (Clarida et al, 2001). However, while these assumptions are analytically convenient, the empirical evidence supports neither. First, because agents can access only a partial set of asset markets, consumption risk cannot be insured completely. In addition, as we have seen earlier, that the degree of exchange rate pass-through is not zero, but neither is it one. In this chapter we relax both of these assumptions and examine their effect on the policy maker's objective function. An additional contribution involves our incorporation of real money balances. Often the role of real balances is ignored in the literature by assuming that its empirical contribution to the analysis will be very small, however, our analysis fully incorporates the analytic role of real balances into the loss function.

The chapter proceeds as follows. Section 3.1 introduces the general framework used to derive the loss function. We begin in section 3.2 with the consumption component of the utility function and add, in section 3.3 , the leisure component of the utility function. Section 3.4 combines the separate parts and in section 3.5 shows how inflation is incorporated into the loss function. Section 3.6 pauses to consider what difference our special assumptions of incomplete asset markets and incomplete passthrough have made to the form of the loss function most often used in the literature. Section 3.7 then adds the role of real balances in the representative household's utility 
function to the loss function. Section 3.8 concludes by focusing on the overall differences that arise in our loss function as compared to the more traditional version.

\subsection{The Basic Framework}

The utility-based loss function derived here extends the analysis of Rotemberg and Woodford (1999) and Woodford (2003) to the open economy under incomplete asset markets and incomplete pass-through as defined in Chapter 2. The basic idea is to take a second-order Taylor series approximation of the representative household's utility function, the welfare measure, around the steady state values of the stationary variables that affect utility. The representative household seeks to maximize the expected value of a discounted sum of period contributions to utility of the form

$$
E_{0}\left[\sum_{i=0}^{\infty} \beta^{t} U_{t}\right]
$$

where $\beta$ is the discount factor, and the period utility function is given by

$$
U_{t}=u\left(C_{t} ; \xi_{t}\right)+u\left(\frac{M_{t}}{P_{t}} ; \xi_{t}\right)-\int_{0}^{1} v\left(n_{t}(i) ; \xi_{t}\right) d i
$$

where $\mathrm{C}_{\mathrm{t}}$ denotes the Dixit-Stiglitz consumption aggregate, $\xi_{t}$ represents exogenous stochastic disturbances to utility, $M_{t} / P_{t}$ the level of real money balances, and $n_{t}(i)$ is the supply of labour of type $i$. Following Woodford (2003), we assume that each of the differentiated goods, indexed by $i$ over the unit interval, uses a specialized labour input in its production. Labour type $i$ is used to produce differentiated good $i$, and the representative household is assumed to supply all types of labour simultaneously. The production function is specified as 


$$
y_{t}(i)=A_{t} f\left(n_{t}(i)\right)
$$

where $A_{t}$ is time varying exogenous technology factor, and $f$ is an increasing, concave function. We first assume that the change in utility coming from changes in real balances will be small so that we can ignore the term $u\left(M_{t} / P_{t} ; \xi_{t}\right)$, and introduce it into the analysis later on. Then using (3.3), we can rewrite (3.2) as

$$
U_{t}=u\left(C_{t} ; \xi_{t}\right)-\int_{0}^{1} \widetilde{v}\left(y_{t}(i) ; \ddot{\xi_{t}}\right) d i
$$

where $\widetilde{v}\left(y_{t}(i) ; \ddot{\xi}_{t}\right)$ is the indirect utility function, defined as

$$
\widetilde{v}\left(y_{t}(i) ; \ddot{\xi}_{t}\right)=v\left(f^{-1}\left(y_{t}(i) / A_{t}\right) ; \xi_{t}\right)
$$

where $\ddot{\xi}_{t}$ includes productivity shocks as well as exogenous disturbances to the direct utility function.

We are now going to approximate in turn each term on the right-hand side of (3.4) by taking its second-order Taylor series expansion. For convenience, we introduce the following notation. For any variable $X_{t}$, let $\bar{X}$ be its steady state value, $X_{t}{ }^{f}$ be the level of the variable under flexible prices (which will also be efficient under our assumption that the government uses an optimal subsidy to offset monopoly power), and $\tilde{X}_{t}=X_{t}-\bar{X}$ be the deviation of $X_{t}$ around its steady state value. Let $\hat{X}_{t}=\ln \left(X_{t} / \bar{X}\right)$ be the log deviation of $X_{t}$ around its steady state value. Taking the antilog of $\hat{X}_{t}$, we obtain $\exp \hat{X}_{t}=X_{t} / \bar{X} . \quad$ Using $\quad$ a $\quad$ second order $\quad$ Taylor series approximation, $\exp \hat{X}_{t}=1+\hat{X}_{t}+\frac{1}{2} \hat{X}_{t}^{2}$. Thus we have the following expression that will be used throughout the following linearizations, 


$$
X_{t}-\bar{X} \approx \bar{X}\left(\hat{X}_{t}+\frac{1}{2} \hat{X}_{t}^{2}\right)
$$

\section{2 The Approximation of $u\left(C_{t} ; \xi_{t}\right)$}

We begin with the first term on the right of $(3.4), u\left(C_{t} ; \xi_{t}\right)$, the level of individual utility derived from consumption. This can be approximated around the steady state as

$$
\begin{aligned}
& u\left(C_{t} ; \xi_{t}\right) \approx u(\bar{C} ; 1)+u_{c}(\bar{C} ; 1)\left(C_{t}-\bar{C}\right)+u_{\xi}(\bar{C} ; 1)\left(\xi_{t}-1\right)+\frac{1}{2} u_{c c}(\bar{C} ; 1)\left(C_{t}-\bar{C}\right)^{2} \\
& +u_{c \xi}(\bar{C} ; 1)\left(C_{t}-\bar{C}\right)\left(\xi_{t}-1\right)+\frac{1}{2} u_{\xi \xi}(\bar{C} ; 0)\left(\xi_{t}-1\right)^{2}+o\left(\|\xi\|^{3}\right)
\end{aligned}
$$

where the steady state value of shocks $\xi_{t}$ is set to one, and $o\left(\|\xi\|^{3}\right)$ denotes that all terms are of third order or higher order. Applying (3.6) to it, it can be rewritten as

$$
\begin{aligned}
& u\left(C_{t} ; \xi_{t}\right) \approx u\left(\bar{C}_{1}\right)+u_{c} \bar{C}\left(\hat{C}_{t}+\frac{1}{2} \hat{C}_{t}^{2}\right)+u_{\xi}\left(\hat{\xi}_{t}+\frac{1}{2} \hat{\xi}_{t}^{2}\right)+\frac{1}{2} u_{c c} \bar{C}^{2}\left(\hat{C}_{t}+\frac{1}{2} \hat{C}_{t}^{2}\right)^{2} \\
& +\frac{1}{2} u_{c \xi} \bar{C}\left(\hat{C}_{t}+\frac{1}{2} \hat{C}_{t}^{2}\right)\left(\hat{\xi}_{t}+\frac{1}{2} \hat{\xi}_{t}^{2}\right)+\frac{1}{2} u_{\xi \xi}\left(\hat{\xi}_{t}+\frac{1}{2} \hat{\xi}_{t}^{2}\right)^{2}+o\left(\|\xi\|^{3}\right) \\
& =u(\bar{C} ; 1)+u_{c} \bar{C}\left(\hat{C}_{t}+\frac{1}{2} \hat{C}_{t}^{2}\right)+u_{\xi}\left(\hat{\xi}_{t}+\frac{1}{2} \hat{\xi}_{t}^{2}\right)+\frac{1}{2} u_{c c} \bar{C}^{2}\left(\hat{C}_{t}^{2}+\hat{C}_{t}^{3}+\frac{1}{4} \hat{C}_{t}^{4}\right), \\
& +\frac{1}{2} u_{c \xi} \bar{C}\left(\hat{C}_{t}+\frac{1}{2} \hat{C}_{t}^{2}\right) \hat{\xi}_{t}+\frac{1}{2} u_{\xi \xi}\left(\hat{\xi}_{t}^{2}+\hat{\xi}_{t}^{3}+\frac{1}{4} \hat{\xi}_{t}^{4}\right)+o\left(\|\xi\|^{3}\right) \\
& \approx u_{c} \bar{C}\left\{\hat{C}_{t}+\frac{1}{2}\left[1+\frac{u_{c c}}{u_{c}} \bar{C}\right] \hat{C}_{t}^{2}+\frac{u_{c \xi}}{u_{c}} \hat{\xi}_{t} \hat{C}_{t}\right\}+t i . p \cdot+o\left(\|\xi\|^{3}\right)
\end{aligned}
$$

where terms involving $\hat{C}_{t}^{i}$ and $\hat{\xi}_{t}{ }^{i}$ for $i>2$ and terms such as $\hat{C}_{t}{ }^{2} \hat{\xi}_{t}$, and $\hat{C}_{t} \hat{\xi}_{t}{ }^{2}$ are ignored. The term, ti.p., denotes terms independent of policy.

Let us define

$$
\sigma=-\frac{u_{c c} \bar{Y}}{u_{c}}
$$


as the coefficient of relative risk aversion, and define

$$
b_{1 t}=-\frac{u_{c \xi}}{u_{c c} \bar{Y}} \xi_{t}
$$

Plugging (3.8) and (3.9) into (3.7), we obtain

$$
u\left(C_{t} ; \xi_{t}\right) \approx u_{c} \bar{C}\left\{\hat{C}_{t}+\frac{1}{2}[1-\sigma] \hat{C}_{t}^{2}+\sigma b_{1 t} \hat{C}_{t}\right\}+t i . p .+o\left(\|\xi\|^{3}\right)
$$

Note that in the steady state the small open economy has zero net exports. This implies that $\bar{C}=\bar{Y}$. Under the assumption of incomplete financial markets and imperfect exchange rate pass-through, the following goods market clearing condition was derived in Chapter 2,

$$
\hat{C}_{t}=\frac{1}{1-\alpha} \hat{Y}_{t}-\frac{\alpha \eta(2-\alpha)}{(1-\alpha)^{2}} \hat{q}_{t}+\frac{\alpha \eta}{(1-\alpha)^{2}} \hat{\Psi}_{t}-\frac{\alpha}{1-\alpha} \hat{C}_{t}^{*}
$$

where $q_{t}$ denotes the real exchange rate, and $\Psi_{t}$, the law of one price gap, which is defined as $\Psi_{t}=\varepsilon_{t} P_{F, t}{ }^{*} / P_{F, t}$, and $C_{t}{ }_{t}^{*}$ is the foreign consumption, which is equal to $Y_{t}^{*}$, the foreign output. Using both the domestic and foreign consumption Euler equations , we obtain

$$
E_{t}\left(\frac{C_{t}}{C_{t+1}}\right)^{-\sigma}=\exp \left(-b N F A_{t}+\ln \chi_{2 t}\right) E_{t}\left(\frac{q_{t+1}}{q_{t}}\right)\left(\frac{C_{t}^{*}}{C_{t+1}^{*}}\right)^{-\sigma}
$$

where following Benigno (2001) and Adolfson (2007), $\exp \left(-b N F A_{t}+\ln \chi_{2 t}\right)$ is the risk premium that the representative household must pay to borrow on world markets-increasing in the size of the net foreign debt held by the representative household-- and where $b$ is a constant governing the size of the risk premium. Here $N F A_{t}=\varepsilon_{t} B_{t}^{*} / P_{t}$ is the domestic value of net foreign assets held by the household, $\varepsilon_{t}$ is the nominal 
exchange rate, defined as the price of foreign currency (in terms of domestic currency), and $\chi_{2 t}$ is the shock to the risk premium. Log-linearizing it and then solving $\hat{C}_{t}$ iterated forward yields

$$
\begin{aligned}
& \hat{C}_{t}=\frac{1}{\sigma} \hat{q}_{t}+\hat{C}_{t}^{*}+\frac{b}{\sigma} N F A_{t}+\frac{1}{\sigma} \hat{\chi}_{2 t}+E_{t}\left[\frac{b}{\sigma} N F A_{t+1}+\frac{1}{\sigma} \hat{\chi}_{2 t+1}\right. \\
& +\frac{b}{\sigma} N F A_{t+2}+\frac{1}{\sigma} \hat{\chi}_{2 t+2}+\cdots+\frac{b}{\sigma} N F A_{t+k}+\frac{1}{\sigma} \hat{\chi}_{2 t+k} \\
& \left.+\hat{C}_{t+k}-\frac{1}{\sigma} \hat{q}_{t+k}-\hat{C}_{t+k}^{*}\right]
\end{aligned}
$$

Then because the small open economy experiences balanced trade in the steady state, $\alpha C_{t+k}{ }^{\sigma}=\alpha^{*} q_{t+k} C_{t+k}{ }^{*}$, where $\alpha$ and $\alpha^{*}$ denotes the steady-state share of imported goods in domestic and foreign household total consumption, respectively. Hence as time goes to infinity, i.e., $k \rightarrow \infty$, we have $\hat{C}_{t+k}=(1 / \sigma) \hat{q}_{t+k}-\hat{C}_{t+k}{ }^{*}$. In addition, the expectation of the $\log$ deviation of shock $\hat{\chi}_{2 t+k}$ at time $t$ is zero. Using these conditions we have

$$
\begin{aligned}
& \hat{C}_{t}=\frac{1}{\sigma} \hat{q}_{t}+\hat{C}_{t}^{*}+\frac{1}{\sigma} \hat{\chi}_{2 t} \\
& +\frac{b}{\sigma} E_{t}\left[N F A_{t}+N F A_{t+1}+N F A_{t+2}+\cdots+N F A_{t+k}\right]
\end{aligned}
$$

Next recall that the net foreign asset position, $N F A_{t}$, is stationary about zero. This then implies that the summation to infinity over all expectations of $N F A_{t+k}$ is zero for $k \geq 0$. Using this condition, we find

$$
\hat{C}_{t}=\frac{1}{\sigma} \hat{q}_{t}+\hat{C}_{t}^{*}+\frac{1}{\sigma} \hat{\chi}_{2 t}
$$


Finally, solving (3.12) for $\hat{C}_{t}{ }^{*}$ and substituting the result in (3.11) yields ${ }^{44}$

$$
\hat{C}_{t}=\hat{Y}_{t}-\Theta \hat{q}_{t}+\frac{\alpha \eta}{1-\alpha} \hat{\Psi}_{t}+\frac{\alpha}{\sigma} \hat{\chi}_{2 t},
$$

where $\Theta=(\phi+\alpha-1) / \sigma(1-\alpha)$ and $\phi=1+\alpha(2-\alpha)(\sigma \eta-1)$. Substituting (3.13) into (3.10), and using $\bar{Y}$ to replace $\bar{C}$, yields

$$
\begin{aligned}
& u\left(C_{t} ; \xi_{t}\right) \approx u_{c} \bar{Y}\left\{\begin{array}{l}
\left(1+\sigma b_{1 t}\right)\left[\hat{Y}_{t}-\Theta \hat{q}_{t}+\frac{\alpha \eta}{1-\alpha} \hat{\Psi}_{t}+\frac{\alpha}{\sigma} \hat{\chi}_{2 t}\right] \\
+\frac{1}{2}(1-\sigma)\left[\hat{Y}_{t}-\Theta \hat{q}_{t}+\frac{\alpha \eta}{1-\alpha} \hat{\Psi}_{t}+\frac{\alpha}{\sigma} \hat{\chi}_{2 t}\right]^{2}
\end{array}\right\} . \\
& + \text { t.i.p. }+o\left(\|\xi\|^{3}\right)
\end{aligned}
$$

Collecting common terms, we obtain

$$
u\left(C_{t} ; \xi_{t}\right) \approx u_{c} \bar{Y}\left\{\begin{array}{l}
\left(1+\sigma b_{1 t}\right) \hat{Y}_{t}+\frac{1}{2}(1-\sigma) \hat{Y}_{t}^{2} \\
-\left(1+\sigma b_{1 t}\right)\left[-\Theta \hat{q}_{t}+\frac{\alpha \eta}{1-\alpha} \hat{\Psi}_{t}\right] \\
+\frac{1}{2}(1-\sigma)\left[\Theta^{2} \hat{q}_{t}^{2}+\left(\frac{\alpha \eta}{1-\alpha}\right)^{2} \hat{\Psi}_{t}^{2}\right] \\
+(1-\sigma)\left[-\Theta \hat{Y}_{t} \hat{q}_{t}+\frac{\alpha \eta}{1-\alpha} \hat{Y}_{t} \hat{\Psi}_{t}+\frac{\alpha}{\sigma} \hat{Y}_{t} \hat{\chi}_{2 t}\right. \\
\left.-\Theta \frac{\alpha \eta}{1-\alpha} \hat{q}_{t} \hat{\Psi}_{t}-\Theta \frac{\alpha}{\sigma} \hat{q}_{t} \hat{\chi}_{2 t}+\frac{\alpha \eta}{1-\alpha} \frac{\alpha}{\sigma} \hat{\Psi}_{t} \hat{\chi}_{2 t}\right]
\end{array}\right\},
$$

Comparing (3.14) with Woodford's (2003) closed economy case, a few new terms have appeared. These include in particular, the real exchange rate and the law of one price gap. This implies that in the open economy the consumption component of utility will depend not only on the deviation of domestic output production from its steady state

\footnotetext{
${ }^{44}$ Note that under the assumptions of complete asset markets and perfect exchange rate pass-through we would have $\hat{C}_{t}=\hat{Y}_{t}-\Theta \hat{q}_{t}$.
} 
but also on the deviation in the relative price differences between the domestic economy and the foreign one.

\subsection{The Approximation of $\int_{0}^{1} \tilde{v}\left(y_{t}(i) ; \ddot{\xi}_{t}\right) d i$}

Next we take the second-order Taylor expansion of $\widetilde{v}\left(y_{t}(i) ; \ddot{\xi}_{t}\right)$ around the steady state values of $\bar{y}$ and $\bar{\xi}=1$.

$$
\begin{aligned}
& \widetilde{v}\left(y_{t}(i) ; \ddot{\xi}_{t}\right) \approx \widetilde{v}(\bar{y} ; 1)+\widetilde{v}_{y}\left(y_{t}(i)-\bar{y}\right)+\widetilde{v}_{\dot{\xi}}\left(\ddot{\xi}_{t}-1\right)+\frac{1}{2} \widetilde{v}_{y y}\left(y_{t}(i)-\bar{y}\right)^{2} \\
& +\widetilde{v}_{y \ddot{\xi}}\left(y_{t}(i)-\bar{y}\right)\left(\ddot{\xi}_{t}-1\right)+\frac{1}{2} \widetilde{v}_{\ddot{\xi} \xi}\left(\ddot{\xi}_{t}-1\right)^{2}+o\left(\|\ddot{\xi}\|^{3}\right)
\end{aligned}
$$

Using (3.6), (3.15) becomes

$$
\begin{aligned}
& \widetilde{v}\left(y_{t}(i) ; \ddot{\xi}_{t}\right) \approx \tilde{v}(\bar{y} ; 1)+\widetilde{v}_{y} \bar{y}\left(\hat{y}_{t}(i)+\frac{1}{2} \hat{y}_{t}(i)^{2}\right)+\widetilde{v}_{\ddot{\xi}}\left(\hat{\xi}_{t}+\frac{1}{2} \hat{\xi}_{t}^{2}\right) \\
& +\frac{1}{2} \widetilde{v}_{y y} \bar{y}^{2}\left(\hat{y}_{t}(i)+\frac{1}{2} \hat{y}_{t}(i)^{2}\right)^{2}+\widetilde{v}_{y \ddot{\xi}} \bar{y}\left(\hat{y}_{t}(i)+\frac{1}{2} \hat{y}_{t}(i)^{2}\right)\left(\hat{\ddot{\xi}}_{t}+\frac{1}{2} \hat{\xi}_{t}^{2}\right), \\
& +\frac{1}{2} \widetilde{v}_{\dot{\xi} \xi}\left(\hat{\ddot{\xi}}_{t}+\frac{1}{2} \hat{\xi}_{t}^{2}\right)^{2}+o\left(\|\ddot{\xi}\|^{3}\right)
\end{aligned}
$$

Ignore terms involving $\hat{y}_{t}^{i}$ for $i>2$ and term $\hat{y}_{t}^{2} \ddot{\xi}_{t}$ and $\hat{y}_{t} \hat{\xi}_{t}^{2}, \quad(3.16)$ can be simplified as

$$
\begin{aligned}
& \widetilde{v}\left(y_{t}(i) ; \ddot{\xi}_{t}\right) \approx \widetilde{v}_{y} \bar{y}\left(\hat{y}_{t}(i)+\frac{1}{2} \hat{y}_{t}(i)^{2}\right)+\frac{1}{2} \widetilde{v}_{y y} \bar{y}^{2}\left(\hat{y}_{t}(i)\right)^{2} \\
& +\widetilde{v}_{y \ddot{\xi}} \bar{y} \hat{y}_{t}(i) \ddot{\xi}_{t}+t . i \cdot p \cdot+o\left(\|\ddot{\xi}\|^{3}\right) \\
& =\tilde{v}_{y} \bar{y}\left[\hat{y}_{t}(i)+\frac{1}{2}\left(1+\frac{\widetilde{v}_{y y}}{\widetilde{v}_{y}} \bar{y}\right) \hat{y}_{t}(i)^{2}+\frac{\widetilde{v}_{y \xi}}{\widetilde{v}_{y}} \ddot{\xi}_{t} \hat{y}_{t}(i)\right] \\
& + \text { t.i.p. }+o\left(\|\ddot{\xi}\|^{3}\right)
\end{aligned}
$$


where t.i.p. include three terms, $\widetilde{v}(\bar{y} ; 0), \widetilde{v}_{\xi} \ddot{\xi}_{t}$, and $\widetilde{v}_{\xi \bar{\xi}}$, which are independent of monetary policy. Let us define

$$
\mu=\frac{\widetilde{v}_{y y}}{\widetilde{v}_{y}} \bar{y}
$$

as the elasticity of real marginal cost with respect to own output $y$, and define

$$
b_{2 t}=-\frac{\widetilde{v}_{y \xi}}{\widetilde{v}_{y y} \ddot{y}_{t}} .
$$

Substituting (3.18) and (3.19) into (3.17), obtain

$$
\begin{aligned}
& \widetilde{v}\left(y_{t}(i) ; \ddot{\xi}_{t}\right) \approx \widetilde{v}_{y} \bar{y}\left(\hat{y}_{t}(i)+\frac{1}{2}\left(1+\frac{\widetilde{v}_{y y}}{\widetilde{v}_{y}} \bar{y}\right) \hat{y}_{t}(i)^{2}+\frac{\widetilde{v}_{y \xi}}{\widetilde{v}_{y}} \xi_{t} \hat{y}_{t}(i)\right)+t \cdot i \cdot p \cdot+o\left(\|\ddot{\xi}\|^{3}\right) \\
& =\widetilde{v}_{y} \bar{y}\left(\hat{y}_{t}(i)+\frac{1}{2}(1+\mu) \hat{y}_{t}(i)^{2}-\mu b_{2 t} \hat{y}_{t}(i)\right)+\text { t.i.p. }+o\left(\|\ddot{\xi}\|^{3}\right)
\end{aligned}
$$

In optimizing, the household equates its marginal rate of substitution between leisure and consumption to the real wage. In our small open economy this implies that

$$
\widetilde{v}_{y}\left(y_{t}(i) ; \ddot{\xi}_{t}\right) / u_{c}\left(C_{t} ; \xi_{t}\right)=W_{t} / P_{t}=W_{t} P_{H, t} / P_{t} P_{H, t}=\varphi_{H, t} P_{H, t} / P_{t},
$$

where $\varphi_{H, t}$ denotes the real marginal cost of production, $P_{t}$, the consumer price index, and $P_{H, t}$, the producer price index ${ }^{45}$. In the presence of monopolistic competition, however,

$$
\widetilde{v}_{y}\left(y_{t}(i) ; \ddot{\xi}_{t}\right) / u_{c}\left(C_{t} ; \xi_{t}\right)=(\theta-1) \varphi_{H, t} P_{H, t} / \theta P_{t},
$$

where $\theta /(\theta-1)$ is the markup arising as the result of producer market power. In Chapter 2 we assumed that the government used a production subsidy $\tau$ to correct the distortion

\footnotetext{
${ }^{45}$ The productivity shock $A_{t}$ is included in $\widetilde{v}_{y}\left(y_{t}(i) ; \ddot{\xi}_{t}\right)$.
} 
arising from the firm's monopolistic competition. The subsidy $\tau$ is set in such a way that the steady-state net markup is zero, that is, $(\theta-1)(1+\tau) / \theta=1$. Using this we have

$$
\tilde{v}_{y}\left(y_{t}(i) ; \ddot{\xi}_{t}\right) / u_{c}\left(C_{t} ; \xi_{t}\right)=\varphi_{H, t} P_{H, t} / P_{t}
$$

In the steady-state (3.21) collapses to $\widetilde{v}_{y} / u_{c}=1$. Using this relationship in (3.20), we obtain

$$
\widetilde{v}\left(y_{t}(i) ; \ddot{\xi}_{t}\right) \approx u_{c} \bar{y}\left(\left(1-\mu b_{2 t}\right) \hat{y}_{t}(i)+\frac{1}{2}(1+\mu) \hat{y}_{t}(i)^{2}\right)+t i, p,+o\left(\|\ddot{\xi}\|^{3}\right)
$$

Integrating it over the differentiated goods $i$ yields

$$
\begin{aligned}
& \int_{0}^{1} \tilde{v}\left(y_{t}(i) ; \ddot{\xi}_{t}\right) d i \approx u_{c} \bar{y}\left(\left(1-\mu b_{2 t}\right) E_{t} \hat{y}_{t}(i)+\frac{1}{2}(1+\mu) E_{t} \hat{y}_{t}(i)^{2}\right)+t \cdot i \cdot p \cdot+o\left(\|\ddot{\xi}\|^{3}\right) \\
& =u_{c} \bar{y}\left(\begin{array}{l}
\left.\left(1-\mu b_{2 t}\right) E_{t} \hat{y}_{t}(i)+\frac{1}{2}(1+\mu)\left(E_{t} \hat{y}_{t}(i)\right)^{2}\right)+t i \cdot p \cdot+o\left(\|\ddot{\xi}\|^{3}\right) \\
+\frac{1}{2}(1+\mu) \operatorname{var}\left(\hat{y}_{t}(i)\right)
\end{array}\right)
\end{aligned}
$$

where the relationship $E_{t} \hat{y}_{t}(i)^{2}=\left(E_{t} \hat{y}_{t}(i)\right)^{2}+\operatorname{var}\left(\hat{y}_{t}(i)\right)$ has been used. To relate individual firm output to the aggregate Dixit-Stiglitz index $Y_{t}$, note that $Y_{t}=\left[\int_{0}^{1} y_{t}(i)^{\frac{\theta-1}{\theta}} d i\right]^{\frac{\theta}{\theta-1}}$. This can be rewritten as $Y_{t}^{\frac{\theta-1}{\theta}}=\int_{0}^{1} y_{t}(i)^{\frac{\theta-1}{\theta}} d i$. Then, dividing $\bar{Y}^{\frac{\theta-1}{\theta}}$ into both sides of it yields

$$
\left(\frac{Y_{t}}{\bar{Y}}\right)^{\frac{\theta-1}{\theta}}=\int_{0}^{1}\left(\frac{y_{t}(i)}{\bar{Y}}\right)^{\frac{\theta-1}{\theta}} d i
$$

Taking the natural logarithms of (3.23),

$$
\frac{\theta-1}{\theta} \ln \left(\frac{Y_{t}}{\bar{Y}}\right)=\ln \int_{0}^{1}\left(\frac{y_{t}(i)}{\bar{Y}}\right)^{\frac{\theta-1}{\theta}} d i
$$


By definition $\hat{Y}_{t}=\ln \left(Y_{t} / \bar{Y}\right),(3.24)$ becomes

$$
\frac{\theta-1}{\theta} \hat{Y}_{t}=\ln \int_{0}^{1}\left(\frac{y_{t}(i)}{\bar{Y}}\right)^{\frac{\theta-1}{\theta}} d i
$$

Using the definition again, $\hat{y}_{t}(i)=\ln \left(y_{t}(i) / \bar{Y}\right)$, and taking the antilog, $y_{t}(i) / \bar{Y}=\exp \hat{y}_{t}(i)$. Raising this expression to the power $(\theta-1) / \theta$ generates

$$
\left(\frac{y_{t}(i)}{\bar{Y}}\right)^{\frac{\theta-1}{\theta}}=\exp \left(\frac{\theta-1}{\theta}\right) \hat{y}_{t}(i) \approx 1+\frac{\theta-1}{\theta} \hat{y}_{t}(i)+\frac{1}{2}\left(\frac{\theta-1}{\theta}\right)^{2} \hat{y}_{t}(i)^{2} .
$$

Integrating the $i$ goods over the unit interval results in

$$
\begin{aligned}
& \int_{0}^{1}\left(\frac{y_{t}(i)}{\bar{Y}}\right)^{\frac{\theta-1}{\theta}} d i \approx \int_{0}^{1}\left(1+\frac{\theta-1}{\theta} \hat{y}_{t}(i)+\frac{1}{2}\left(\frac{\theta-1}{\theta}\right)^{2} \hat{y}_{t}(i)^{2}\right) d i \\
& =1+\frac{\theta-1}{\theta} E_{t} \hat{y}_{t}(i)+\frac{1}{2}\left(\frac{\theta-1}{\theta}\right)^{2} E_{t} \hat{y}_{t}(i)^{2}
\end{aligned}
$$

Taking the $\log$ on (3.27) obtain

$$
\begin{aligned}
& \ln \int_{0}^{1}\left(\frac{y_{t}(i)}{\bar{Y}}\right)^{\frac{\theta-1}{\theta}} d i \approx \ln \left(1+\frac{\theta-1}{\theta} E_{t} \hat{y}_{t}(i)+\frac{1}{2}\left(\frac{\theta-1}{\theta}\right)^{2} E_{t} \hat{y}_{t}(i)^{2}\right) \\
& \approx \frac{\theta-1}{\theta} E_{t} \hat{y}_{t}(i)+\frac{1}{2}\left(\frac{\theta-1}{\theta}\right)^{2} E_{t} \hat{y}_{t}(i)^{2} \\
& =\frac{\theta-1}{\theta} E_{t} \hat{y}_{t}(i)+\frac{1}{2}\left(\frac{\theta-1}{\theta}\right)^{2}\left[\operatorname{var}_{i}\left(\hat{y}_{t}(i)\right)+\left(E_{t} \hat{y}_{t}(i)\right)^{2}\right] \\
& =\frac{\theta-1}{\theta} E_{t} \hat{y}_{t}(i)+\frac{1}{2}\left(\frac{\theta-1}{\theta}\right)^{2} \operatorname{var}_{i}\left(\hat{y}_{t}(i)\right)
\end{aligned}
$$

where last equality is obtained after ignoring the term $\left(E_{t} \hat{y}_{t}(i)\right)^{2}$ since it is very small. Combining (3.28) with (3.25) yields

$$
\hat{Y}_{t} \approx E_{t} \hat{y}_{t}(i)+\frac{1}{2}\left(\frac{\theta-1}{\theta}\right) \operatorname{var}_{i}\left(\hat{y}_{t}(i)\right)
$$


and after substituting (3.29) into (3.22), we obtain

$$
\int_{0}^{1} \widetilde{v}\left(y_{t}(i) ; \ddot{\xi_{t}}\right) d i \approx u_{c} \bar{y}\left(\begin{array}{l}
\left(1-\mu b_{2 t}\right)\left(\hat{Y}_{t}-\frac{1}{2} \frac{\theta-1}{\theta} \operatorname{var}_{i}\left(\hat{y}_{t}(i)\right)\right) \\
+\frac{1}{2}(1+\mu)\left(\hat{Y}_{t}-\frac{1}{2} \frac{\theta-1}{\theta} \operatorname{var}_{i}\left(\hat{y}_{t}(i)\right)\right)^{2} \operatorname{var}_{i}\left(\hat{y}_{t}(i)\right)
\end{array}\right)+t i \cdot p \cdot+o\left(\|\dot{\xi}\|^{3}\right) .
$$

Finally after collecting common terms, and ignoring terms such as $\hat{Y}_{t} \operatorname{var}_{i}\left(\hat{y}_{t}\right)$, $\left(\operatorname{var}_{i} \hat{y}_{t}(i)\right)^{2}$, and $\mu b_{2 t}\left(\frac{1}{2} \frac{\theta-1}{\theta} \operatorname{var}_{i}\left(\hat{y}_{t}\right)\right),(3.30)$ becomes

$$
\int_{0}^{1} \tilde{v}\left(y_{t}(i) ; \ddot{\xi}_{t}\right) d i \approx u_{c} \hat{y}\left(\begin{array}{l}
\left(1-\mu b_{2 t}\right) \hat{Y}_{t}+\frac{1}{2}(1+\mu) \hat{Y}_{t}^{2} \\
+\frac{1}{2}(1+\mu) \operatorname{var}_{i}\left(\hat{y}_{t}(i)\right)
\end{array}\right)+t i \cdot \cdot \cdot \cdot+o\left(\|\ddot{\xi}\|^{3}\right) .
$$

\subsection{Combining the Linear Approximations of $u\left(C_{t} ; \xi_{t}\right)$ with $\int_{0}^{1} \widetilde{v}\left(y_{t}(i) ; \ddot{\xi}_{t}\right) d i$}

The linear approximation of (3.4) then requires the combining of (3.14) with (3.31), i.e.,

$$
\begin{aligned}
& U_{t}=u\left(C_{t} ; \xi_{t}\right)-\int_{0}^{1} \tilde{v}\left(y_{t}(i) ; \ddot{\xi}_{t}\right) d i \\
& \approx u_{c} \bar{Y}\left\{\begin{array}{l}
\left(1+\sigma b_{1 t}\right) \hat{Y}_{t}+\frac{1}{2}(1-\sigma) \hat{Y}_{t}^{2}-\left(1+\sigma b_{1 t}\right)\left[-\Theta \hat{q}_{t}+\frac{\alpha \eta}{1-\alpha} \hat{\Psi}_{t}\right] \\
+\frac{1}{2}(1-\sigma)\left[\Theta^{2} \hat{q}_{t}^{2}+\left(\frac{\alpha \eta}{1-\alpha}\right)^{2} \hat{\Psi}_{t}^{2}\right]+(1-\sigma)\left[-\Theta \hat{Y}_{t} \hat{q}_{t}+\frac{\alpha \eta}{1-\alpha} \hat{Y}_{t} \hat{\Psi}_{t}\right. \\
\left.+\frac{\alpha}{\sigma} \hat{Y}_{t} \hat{\chi}_{2 t}-\Theta \frac{\alpha \eta}{1-\alpha} \hat{q}_{t} \hat{\Psi}_{t}-\Theta \frac{\alpha}{\sigma} \hat{q}_{t} \hat{\chi}_{2 t}+\frac{\alpha \eta}{1-\alpha} \frac{\alpha}{\sigma} \hat{\Psi}_{t} \hat{\chi}_{2 t}\right]
\end{array}\right\} . \\
& -u_{c} \bar{y}\left(\begin{array}{l}
\left(1-\mu b_{2 t}\right) \hat{Y}_{t}+\frac{1}{2}(1+\mu) \hat{Y}_{t}^{2} \\
+\frac{1}{2}(1+\mu) \operatorname{var}_{i}\left(\hat{y}_{t}(i)\right)
\end{array}\right)+\text { ti. } . p .+o\left(\|\ddot{\xi}\|^{3}\right)
\end{aligned}
$$

Collecting terms yields 


$$
\begin{aligned}
& U_{t}=u\left(C_{t} ; \xi_{t}\right)-\int_{0}^{1} \tilde{v}\left(y_{t}(i) ; \ddot{\xi}_{t}\right) d i \\
& \approx u_{c} \bar{Y}\left\{\begin{array}{l}
\left(\sigma b_{1 t}+\mu b_{2 t}\right) \hat{Y}_{t}-\frac{1}{2}(\mu+\sigma) \hat{Y}_{t}^{2}-\frac{1}{2}(1+\mu) \operatorname{var}_{i}\left(\hat{y}_{t}(i)\right) \\
-\left(1+\sigma b_{1 t}\right)\left[-\Theta \hat{q}_{t}+\frac{\alpha \eta}{1-\alpha} \hat{\Psi}_{t}\right]+\frac{1}{2}(1-\sigma)\left[\Theta^{2} \hat{q}_{t}^{2}+\left(\frac{\alpha \eta}{1-\alpha}\right)^{2} \hat{\Psi}_{t}^{2}\right] \\
+(1-\sigma)\left[-\Theta \hat{Y}_{t} \hat{q}_{t}+\frac{\alpha \eta}{1-\alpha} \hat{Y}_{t} \hat{\Psi}_{t}+\frac{\alpha}{\sigma} \hat{Y}_{t} \hat{\chi}_{2 t}-\Theta \frac{\alpha \eta}{1-\alpha} \hat{q}_{t} \hat{\Psi}_{t}\right. \\
\left.-\Theta \frac{\alpha}{\sigma} \hat{q}_{t} \hat{\chi}_{2 t}+\frac{\alpha \eta}{1-\alpha} \frac{\alpha}{\sigma} \hat{\Psi}_{t} \hat{\chi}_{2 t}\right] \\
+ \text { t.i.p. }+o\left(\|\xi\|^{3}\right)
\end{array}\right\}
\end{aligned}
$$

and taking the term $-\frac{1}{2}(\sigma+\mu)$ out of the curled bracket, we obtain

$$
U_{t} \approx-\frac{1}{2}(\mu+\sigma) u_{c} \bar{Y}\left\{\begin{array}{l}
\hat{Y}_{t}^{2}-2 \frac{\sigma b_{1 t}+\mu b_{2 t}}{\mu+\sigma} \hat{Y}_{t}-\frac{1+\mu}{\mu+\sigma} \operatorname{var}_{i}\left(\hat{y}_{t}(i)\right) \\
-\frac{1-\sigma}{\mu+\sigma}\left[\Theta^{2} \hat{q}_{t}^{2}+\left(\frac{\alpha \eta}{1-\alpha}\right)^{2} \hat{\Psi}_{t}^{2}\right]-2 \frac{1+\sigma b_{1 t}}{\mu+\sigma}\left[-\Theta \hat{q}_{t}\right. \\
\left.+\frac{\alpha}{1-\alpha}\right] \\
-2 \frac{1-\sigma}{\mu+\sigma}\left[-\Theta \hat{Y}_{t} \hat{q}_{t}+\frac{\alpha \eta}{1-\alpha} \hat{Y}_{t} \hat{\Psi}_{t}+\frac{\alpha}{\sigma} \hat{Y}_{t} \hat{\chi}_{2 t}\right. \\
\left.-\Theta \frac{\alpha \eta}{1-\alpha} \hat{q}_{t} \hat{\Psi}_{t}-\Theta \frac{\alpha}{\sigma} \hat{q}_{t} \hat{\chi}_{2 t}+\frac{\alpha \eta}{1-\alpha} \frac{\alpha}{\sigma} \hat{\Psi}_{t} \hat{\chi}_{2 t}\right]
\end{array}\right\}
$$

Equation (3.32) indicates that the representative household's welfare is adversely affected by the $\log$ deviation of output from its steady state, the variance of firms' output, $\operatorname{var}_{i} \hat{y}_{t}(i)$, the deviation of the real exchange rate, and the law of one price gap. In order to gain more insight into the elements of welfare that can be affected by the central bank, it is more useful to characterize welfare losses in terms of the deviation in output 
from the equilibrium that would arise under flexible rather than sticky prices. To do so again note that in the flexible price equilibrium (under monopolistic competition with an optimal subsidy), the marginal product of labour will always equal the marginal rate of substitution between leisure and consumption. In the analysis framework (a small open economy) used earlier in the thesis this implies

$$
\frac{\widetilde{v}_{y}\left(y_{t} ; \ddot{\xi}_{t}\right)}{u_{c}\left(C_{t} ; \xi_{t}\right)}=\frac{P_{H, t}}{P_{t}} \bar{\varphi}_{H, t},
$$

where the real marginal cost of production, $\bar{\varphi}_{H, t}$, is a constant, so that $y_{t}$ equals the flexible price output level $Y_{t}^{f}$ satisfying (3.33). Taking the first-order Taylor series expansion of (3.34), we have

$$
\begin{aligned}
& \bar{P}_{t} \widetilde{v}_{y}(\bar{y} ; 1)+\bar{P}_{t} \widetilde{v}_{y y}\left(Y_{t}^{f}-\bar{Y}\right)+\bar{P}_{t} \widetilde{v}_{y \xi}\left(\ddot{\xi}_{t}-1\right)+\widetilde{v}_{y}(\bar{y} ; 1)\left(P_{t}-\bar{P}_{t}\right) \\
& =\bar{P}_{H, t} u_{c}(\bar{C} ; 1)+\bar{P}_{H, t} u_{c c}\left(C_{t}-\bar{C}\right)+\bar{P}_{H, t} u_{c \xi}\left(\xi_{t}-1\right)+u_{c}(\bar{C} ; 1)\left(P_{H, t}-\bar{P}_{H, t}\right)
\end{aligned}
$$

Dividing both sides of (3.34) by $\bar{P}_{t}\left[\widetilde{v}_{y}(\bar{y} ; 1)\right]=\bar{P}_{H, t} u_{c}(\bar{C} ; 1)$ yields

$$
\frac{\widetilde{v}_{y y}}{\widetilde{v}_{y}} \bar{Y} \hat{Y}_{t}^{f}+\frac{\widetilde{v}_{y \xi} \ddot{\xi}_{t}}{\widetilde{v}_{y}}=\frac{u_{c c}}{u_{c}} \bar{C} \hat{C}_{t}+\frac{u_{c \xi} \xi_{t}}{u_{c}}+\hat{P}_{H, t}-\hat{P}_{t} .
$$

Applying (3.8), (3.9), (3.13), (3.18), (3.19), and the condition $\bar{C}=\bar{Y},(3.35)$ can be rewritten as

$$
\mu \hat{Y}_{t}^{f}-\mu b_{2 t}=-\sigma\left[\hat{Y}_{t}^{f}-\Theta \hat{q}_{t}^{f}+\frac{\alpha \eta}{1-\alpha} \hat{\Psi}_{t}^{f}+\frac{\alpha}{\sigma} \hat{\chi}_{2 t}\right]+\sigma b_{1 t}-\frac{\alpha}{(1-\alpha)} \hat{q}_{t}^{f} .
$$


Note that under the assumptions of perfectly flexible prices and complete asset markets, $\hat{\Psi}_{t}^{f}=0$, and $\hat{\chi}_{2 t}=0$. Collecting common terms and rearranging it to solve for $\hat{Y}_{t}^{f 46}$,

$$
\hat{Y}_{t}^{f}=\frac{\sigma b_{1 t}+\mu b_{2 t}}{\sigma+\mu}+\frac{\phi-1}{(\sigma+\mu)(1-\alpha)} \hat{q}_{t}^{f}
$$

We now turn back to (3.32). Note that the first two terms in the curled bracket of (3.32) can be written as by subtracting and adding $2\left(\frac{\phi-1}{(\sigma+\mu)(1-\alpha)} \hat{q}_{t}^{f}\right) \hat{Y}_{t}$,

$$
\hat{Y}_{t}^{2}-2\left(\frac{\sigma b_{1 t}+\mu b_{2 t}}{\sigma+\mu}+\frac{\phi-1}{(\sigma+\mu)(1-\alpha)} \hat{q}_{t}{ }^{f}\right) \hat{Y}_{t}+2\left(\frac{\phi-1}{(\sigma+\mu)(1-\alpha)} \hat{q}_{t}{ }^{f}\right) \hat{Y}_{t}
$$

Using (3.36), we can write (3.37) as $\hat{Y}_{t}^{2}-2 \hat{Y}_{t}^{f} \hat{Y}_{t}+2\left(\frac{\phi-1}{(\sigma+\mu)(1-\alpha)}\right) \hat{Y}_{t} \hat{q}_{t}^{f}$. After adding and subtracting some more terms, we finally obtain

$$
x_{t}^{2}+2\left(\frac{\phi-1}{(\sigma+\mu)(1-\alpha)}\right) \hat{Y}_{t} \hat{q}_{t}^{f}-\left(\hat{Y}_{t}^{f}\right)^{2},
$$

where $x_{t}=\hat{Y}_{t}-\hat{Y}_{t}^{f}$. Using (3.38) to replace the first two terms in the curled bracket of (3.32) gives

${ }^{46}$ Recall that the representative household's period utility function is specified as

$$
u\left(C_{t} \frac{M_{t}}{P_{t}}, N_{t}, \chi_{1 t}\right)=\frac{\chi_{1 t}}{1-\sigma} C_{t}^{1-\sigma}+\frac{1}{1-\varsigma}\left(\frac{M_{t}}{P_{t}}\right)^{1-\varsigma}-\frac{1}{1+\mu} \int_{0}^{1}\left(N_{t}(i)\right)^{1+\mu} d i
$$

and production function is given by equation (3.3). Using (3.9) and (3.19) we have $\sigma b_{1 t}=\hat{\chi}_{1 t}$, and $\mu b_{2 t}=(1+\mu) \hat{A}_{t}$. Applying these conditions into (3.36) gives $\hat{Y}_{t}^{f}=\frac{\phi-1}{(\sigma+\mu)(1-\alpha)} \hat{q}_{t}^{f}+\frac{1+\mu}{\sigma+\mu} \hat{A}_{t}+\frac{1+\mu}{\sigma+\mu} \hat{\chi}_{1 t}$. 


$$
U_{t} \approx-\frac{1}{2}(\sigma+\mu) u_{c} \bar{Y}\left\{\begin{array}{l}
x_{t}{ }^{2}+\frac{1+\mu}{\sigma+\mu} \operatorname{var}_{i}\left(\hat{y}_{t}(i)\right)+2\left(\frac{\phi-1}{(\sigma+\mu)(1-\alpha)}\right) \hat{Y}_{t} \hat{q}_{t}^{f} \\
-\frac{1-\sigma}{\mu+\sigma}\left[\Theta^{2} \hat{q}_{t}{ }^{2}+\left(\frac{\alpha \eta}{1-\alpha}\right)^{2} \hat{\Psi}_{t}^{2}\right] \\
-2 \frac{1+\sigma b_{1 t}}{\mu+\sigma}\left[-\Theta \hat{q}_{t}+\frac{\alpha \eta}{1-\alpha} \hat{\Psi}_{t}\right] \\
-2 \frac{1-\sigma}{\mu+\sigma}\left[-\Theta \hat{Y}_{t} \hat{q}_{t}+\frac{\alpha \eta}{1-\alpha} \hat{Y}_{t} \hat{\Psi}_{t}+\frac{\alpha}{\sigma} \hat{Y}_{t} \hat{\chi}_{2 t}\right. \\
\left.-\Theta \frac{\alpha \eta}{1-\alpha} \hat{q}_{t} \hat{\Psi}_{t}-\Theta \frac{\alpha}{\sigma} \hat{q}_{t} \hat{\chi}_{2 t}+\frac{\alpha \eta}{1-\alpha} \frac{\alpha}{\sigma} \hat{\Psi}_{t} \hat{\chi}_{2 t}\right]
\end{array}\right\}
$$

The approximation of the representative household's utility when both consumption and leisure are included is given in (3.39). Two points are worth mentioning. First, the representative household's utility is affected by only a few distinct factors: the output gap, the dispersion of output levels across sectors, and the real exchange rate. Second, in the small open economy relative prices, such as the nominal exchange rate, real exchange rate and the terms of trade, really matter.

\subsection{Tying the $\operatorname{Var}_{i}\left(\hat{y}_{t}(i)\right)$ to the Inflation Rate}

From earlier thesis work, we have shown that demand for good $i$ can be expressed as

$$
y_{t}(i)=\left(\frac{p_{H, t}(i)}{P_{H, t}}\right)^{-\theta} Y_{t} .
$$

Dividing (3.40) by $\bar{Y}$ and taking natural logarithms,

$$
\ln \frac{y_{t}(i)}{\bar{Y}}=\ln \frac{Y_{t}}{\bar{Y}}-\theta\left(\ln p_{H, t}(i)-\ln P_{H, t}\right)
$$

Then by definition, we can rewrite (3.41) as 


$$
\hat{y}_{t}(i)=\ln \frac{Y_{t}}{\bar{Y}}-\theta\left(\ln p_{H, t}(i)-\ln P_{H, t}\right),
$$

and taking the variance over $i$ with respect to good $i$, we obtain

$$
\operatorname{var}_{i} \hat{y}_{t}(i)=\theta^{2} \operatorname{var}_{i} \ln p_{H, t}(i) \text {. }
$$

Substituting (3.42) into (3.39) yields

$$
U_{t} \approx-\frac{1}{2}(\sigma+\mu) u_{\sigma} \bar{Y}\left\{\begin{array}{l}
\left(x_{t}\right)^{2}+\frac{1+\mu}{\sigma+\mu} \theta^{2} \operatorname{var}_{i} \ln p_{H, t}(i)-\frac{1-\sigma}{\mu+\sigma}\left[\Theta^{2} \hat{q}_{t}{ }^{2}\right. \\
\left.+\left(\frac{\alpha \eta}{1-\alpha}\right)^{2} \hat{\Psi}_{t}^{2}\right]-2 \frac{1+\sigma b_{1 t}}{\mu+\sigma}\left[-\Theta \hat{q}_{t}+\frac{\alpha \eta}{1-\alpha} \hat{\Psi}_{t}\right] \\
-2 \frac{1-\sigma}{\mu+\sigma}\left[-\Theta \hat{Y}_{t} \hat{q}_{t}+\frac{\alpha \eta}{1-\alpha} \hat{Y}_{t} \hat{\Psi}_{t}+\frac{\alpha}{\sigma} \hat{Y} \hat{\chi}_{2 t}\right. \\
\left.-\Theta \frac{\alpha \eta}{1-\alpha} \hat{q}_{t} \hat{\Psi}_{t}-\Theta \frac{\alpha}{\sigma} \hat{q}_{t} \hat{\chi}_{2 t}+\frac{\alpha \eta}{1-\alpha} \frac{\alpha}{\sigma} \hat{\Psi}_{t} \hat{\chi}_{2 t}\right] \\
+2\left(\frac{\phi-1}{(\sigma+\mu)(1-\alpha)}\right) \hat{Y}_{t} \hat{q}_{t}^{f}
\end{array}\right\}
$$

Define $\bar{P}_{H, t}=E_{i} \ln p_{H, t}(i)$, and $\Delta_{t}=\operatorname{var}_{i} \ln p_{H, t}(i)$. Since $\operatorname{var}_{i} \bar{P}_{H, t-1}=0$, we can write

$$
\begin{aligned}
& \Delta_{t}=\operatorname{var}_{i}\left(\ln p_{H, t}(i)-\bar{P}_{H, t-1}\right) \\
& =E_{i}\left(\ln p_{H, t}(i)-\bar{P}_{H, t-1}\right)^{2}-\left[E_{i}\left(\ln p_{H, t}(i)-\bar{P}_{H, t-1}\right)\right]^{2} . \\
& =E_{i}\left(\ln p_{H, t}(i)-\bar{P}_{H, t-1}\right)^{2}-\left(E_{i} \ln p_{H, t}(i)-\bar{P}_{H, t-1}\right)^{2}
\end{aligned}
$$

By Calvo price setting specification, (3.44) can be written as

$$
\begin{aligned}
& \Delta_{t}=\omega E_{i}\left(\ln p_{H, t-1}(i)-\bar{P}_{H, t-1}\right)^{2}+(1-\omega)\left(\ln p_{H, t}{ }^{n}-\bar{P}_{H, t-1}\right)^{2}, \\
& -\left(E_{i} \ln p_{H, t}(i)-\bar{P}_{H, t-1}\right)^{2}
\end{aligned}
$$

where $p_{H, t}{ }^{n}$ is the new price set at time $\mathrm{t}$ by the fraction $1-\omega$ of firms. Given that $E_{i} \ln p_{H, t}(i)=(1-\omega) \ln p_{H, t}{ }^{n}+\omega \bar{P}_{H, t-1}$. Subtracting $\bar{P}_{H, t-1}$ on both sides yields 


$$
E_{i} \ln p_{H, t}(i)-\bar{P}_{H, t-1}=(1-\omega)\left(\ln p_{H, t}{ }^{n}-\bar{P}_{H, t-1}\right)
$$

This gives us

$$
\left(\ln p_{H, t}{ }^{n}-\bar{P}_{H, t-1}\right)=\frac{1}{1-\omega}\left(E_{i} \ln p_{H, t}(i)-\bar{P}_{H, t-1}\right)
$$

Substituting (3.46) into (3.45) yields

$$
\begin{gathered}
\Delta_{t}=\omega E_{i}\left(\ln p_{H, t-1}(i)-\bar{P}_{H, t-1}\right)^{2}+\frac{1}{(1-\omega)}\left(E_{i} \ln p_{H, t}(i)-\bar{P}_{H, t-1}\right)^{2} \\
-\left(E_{i} \ln p_{H, t}(i)-\bar{P}_{H, t-1}\right)^{2} \\
=\omega \operatorname{var}_{i}\left(\ln p_{H, t-1}(i)-\bar{P}_{H, t-1}\right)+\omega\left[E_{i}\left(\ln p_{H, t-1}(i)-\bar{P}_{H, t-1}\right)\right]^{2} \\
+\frac{\omega}{(1-\omega)}\left(E_{i} \ln p_{H, t}(i)-\bar{P}_{H, t-1}\right)^{2}
\end{gathered}
$$

Simplifying it yields

$$
\Delta_{t}=\omega \Delta_{t-1}+\frac{\omega}{(1-\omega)}\left(\hat{\Pi}_{H, t}\right)^{2}
$$

Given (3.47), the present value of $\Delta_{t+1}$ can be written as

$$
\beta \Delta_{t+1}=\omega \beta \Delta_{t}+\frac{\omega}{(1-\omega)} \beta\left(\hat{\Pi}_{H, t+1}\right)^{2}
$$

Similarly, the present value of $\Delta_{t+i}$ is $\beta^{i} \Delta_{t+i}=\omega \beta^{i-1} \Delta_{t}+\frac{\omega}{(1-\omega)} \beta^{i}\left(\hat{\Pi}_{H, t+i}\right)^{2}$ as $i$ goes to infinity. Summing the $\Delta_{t+i}$ from $\mathrm{i}=0$ to infinity, we obtain

$$
\begin{aligned}
& \Delta_{t}+\beta \Delta_{t+1}+\beta^{2} \Delta_{t+2}+\cdots+\beta^{i} \Delta_{t+i} \\
& =\omega \Delta_{t-1}+\frac{\omega}{1-\omega} \hat{\Pi}_{H, t}{ }^{2}+\omega \beta \Delta_{t}+\frac{\omega}{1-\omega} \beta \hat{\Pi}_{H, t+1}{ }^{2} \\
& +\omega \beta^{2} \Delta_{t+1}+\frac{\omega}{1-\omega} \beta^{2} \hat{\Pi}_{H, t+2}{ }^{2}+\cdots \\
& +\omega \beta^{i} \Delta_{t+i-1}+\frac{\omega}{1-\omega} \beta^{i} \hat{\Pi}_{H, t+i}{ }^{2}
\end{aligned}
$$

Collecting common terms, obtain 


$$
\begin{aligned}
& E_{t} \sum_{i=0}^{\infty} \beta^{i} \Delta_{t+i}=\frac{\omega}{1-\omega \beta} \Delta_{t-1}+\frac{\omega}{(1-\omega)(1-\omega \beta)} E_{t} \sum_{i=0}^{\infty} \beta^{i} \hat{\Pi}_{H, t}{ }^{2} \\
& =\frac{\omega}{(1-\omega)(1-\omega \beta)} E_{t} \sum_{i=0}^{\infty} \beta^{i} \hat{\Pi}_{H, t+i}{ }^{2}+t . i . p .
\end{aligned}
$$

since $\frac{\omega}{1-\omega \beta} \Delta_{t-1}$ is independent of policy.

\subsection{The Period Loss Function without the Real Balances}

From (3.43), the present discounted value of the utility of the representative household as

$$
\left.\begin{array}{l}
E_{t} \sum_{i=0}^{\infty} \beta^{i} U_{t+i} \approx-\frac{1}{2}(\mu+\sigma) u_{c} \bar{Y} \\
x_{t+i}{ }^{2}+\frac{1+\mu}{\mu+\sigma} \theta^{2} \operatorname{var}_{i} \ln p_{H, t}(t+i) \\
-\frac{1-\sigma}{\mu+\sigma}\left[\Theta^{2} \hat{q}_{t+i}^{2}+\left(\frac{\alpha \eta}{1-\alpha}\right)^{2} \hat{\Psi}_{t+i}^{2}\right] \\
E_{t} \sum_{i=0}^{\infty} \beta^{i}\left\{\begin{array}{l}
-2 \frac{1}{\mu+\sigma}\left[-\Theta \hat{q}_{t+i}+\frac{\alpha \eta}{1-\alpha} \hat{\Psi}_{t+i}\right]-2 \frac{\sigma b_{1 t+i}}{\mu+\sigma}\left[-\Theta \hat{q}_{t+i}+\frac{\alpha \eta}{1-\alpha} \hat{\Psi}_{t+i}\right] \\
-2 \frac{1-\sigma}{\mu+\sigma}\left[-\Theta \hat{Y}_{t+i} \hat{q}_{t+i}+\frac{\alpha \eta}{1-\alpha} \hat{Y}_{t+i} \hat{\Psi}_{t+i}+\frac{\alpha}{\sigma} \hat{Y}_{t+i} \hat{\chi}_{2 t+i}\right. \\
\left.-\Theta \frac{\alpha \eta}{1-\alpha} \hat{q}_{t+i} \hat{\Psi}_{t+i}-\Theta \frac{\alpha}{\sigma} \hat{q}_{t+i} \hat{\chi}_{2 t+i}+\frac{\alpha \eta}{1-\alpha} \frac{\alpha}{\sigma} \hat{\Psi}_{t+i} \hat{\chi}_{2 t+i}\right] \\
+2\left(\frac{\phi-1}{(\mu+\sigma)(1-\alpha)}\right) \hat{Y}_{t+i} \hat{q}_{t+i}^{f}
\end{array}\right. \\
+t . i . p .+o\left(\|\ddot{\xi}\|^{3}\right)
\end{array}\right\}
$$

Since $\hat{q}_{t}$ and $\hat{\Psi}_{t}$ are stationary processes, expectations over them are zero, thus the term $\frac{2}{\mu+\sigma} E_{t}\left[\Theta \hat{q}_{t+i}-\frac{\alpha \eta}{1-\alpha} \hat{\Psi}_{t+i}\right]$ can be dropped from (3.49). To write (3.49) in 
terms of gaps we first use (3.48) to substitute for $E_{t} \sum_{t=0}^{\infty} \beta^{i} \operatorname{var}_{i} \ln p_{H, t}(t+i)$ into (3.49), and then add and subtract terms, such as $2 \frac{\sigma-1}{\mu+\sigma} \Theta^{2} \hat{q}_{t+i} \hat{q}_{t+i}{ }^{f}$, this gives

$$
\left.\begin{array}{l}
E_{t} \sum_{i=0}^{\infty} \beta^{i} U_{t+i} \approx-\frac{1}{2} u_{c} \bar{Y} \\
E_{t} \sum_{i=0}^{\infty} \beta^{i}\left\{\begin{array}{l}
(\mu+\sigma) x_{t+i}{ }^{2}+\frac{\omega(1+\mu) \theta^{2}}{(1-\omega)(1-\omega \beta)} \hat{\Pi}_{H, t+i}{ }^{2}+(\sigma-1)\left[\Theta^{2} Q g_{t+i}{ }^{2}\right. \\
\left.+\left(\frac{\alpha \eta}{1-\alpha}\right)^{2} \hat{\Psi}_{t+i}{ }^{2}\right]+2(\sigma-1)\left[-\Theta x_{t+i} Q g_{t+i}+\frac{\alpha \eta}{1-\alpha} x_{t+i} \hat{\Psi}_{t+i}{ }^{2}\right. \\
\left.-\Theta \frac{\alpha \eta}{1-\alpha} Q g_{t+i} \hat{\Psi}_{t+i}\right]+2\left[\Theta Q g_{t+i} \hat{\chi}_{1 t+i}-\frac{\alpha \eta}{1-\alpha} \hat{\Psi}_{t+i} \hat{\chi}_{1 t+i}\right] \\
+2\left(\frac{\sigma(\phi-1)}{\sigma}\left[x_{t+i} \hat{\chi}_{2 t+i}-\Theta Q g_{t+i} \hat{\chi}_{2 t+i}+\frac{\alpha \eta}{1-\alpha} \hat{\Psi}_{t+i} \hat{\chi}_{2 t+i}\right]\right. \\
\sigma(\sigma-1)(\phi+\alpha-1) \\
\sigma(1-\alpha)
\end{array} x_{t+i} \hat{q}_{t+i}{ }^{f}\right. \\
-2(\sigma-1)\left(\begin{array}{c}
\Theta Q g_{t+i} \hat{Y}_{t+i}{ }^{f}+\frac{\alpha \eta}{1-\alpha} \hat{\Psi}_{t+i} \hat{Y}_{t+i}{ }^{f} \\
-\Theta \frac{\alpha \eta}{1-\alpha} \hat{\Psi}_{t+i} \hat{q}_{t+i}{ }^{f}-\Theta^{2} Q g_{t+i} \hat{q}_{t+i}{ }^{f}
\end{array}\right) \\
+ \text { t.i.p. }+o\left(\|\ddot{\xi}\|^{3}\right)
\end{array}\right\}
$$

where the condition $\sigma b_{1 t}=\hat{\chi}_{1 t}$ has been used, and $Q g_{t+i}=\hat{q}_{t+i}-\hat{q}_{t+i}{ }^{f}$.

As is well known, in the case of a closed economy, a loss function derived from the household utility function can be derived solely in terms of two gaps, the output gap $x_{t}$ and the inflation gap, $\hat{\Pi}_{H, t}-\hat{\Pi}_{H, t}{ }^{f}$ where $\hat{\Pi}_{H, t}{ }^{f}$ is set to zero (see, e.g., Woodford, 1999). Here we can obtain exactly the same function from (3,50) by imposing that $\alpha=0$. Note that when $\alpha=0, \phi=1$ and $\Theta=0$. Here we get 


$$
\begin{aligned}
& E_{t} \sum_{i=0}^{\infty} \beta^{i} U_{t+i} \approx-\frac{1}{2}(1+\mu) u_{c} \vec{Y} \\
& E_{t} \sum_{i=0}^{\infty} \beta^{i}\left\{x_{t+i}{ }^{2}+\frac{\omega \theta^{2}}{(1-\omega)(1-\omega \beta)} \hat{\Pi}_{H, t+i}{ }^{2}\right\} \\
& + \text { t.i.p. }+o\left(\|\ddot{\xi}\|^{3}\right)
\end{aligned}
$$

In addition, Gali and Monacelli (2002) use a special form for the utility function to derive their loss function for a small open economy. Assuming log utility, which implies in our case that $\sigma=1$ and also assuming that the elasticity of substitution between domestic and foreign goods is one, i.e., $\eta=1$, and that both asset markets and exchange rate passthrough are complete, they find that the policy maker's objective function in a small open economy can also be written in terms of these two gaps, and is therefore isomorphic to the closed economy case. ${ }^{47}$ If we apply all these assumptions to our analysis, we find that $\phi=1, \Theta=\alpha /(1-\alpha), \hat{\Psi}_{t}=0$, and $\chi_{1 t}=0$ so that (3.50) collapses to (3.51) and replicates Gali and Monacelli's (2002) result. Note, however, that when even a preference shock, $\chi_{1 t}$, is included in the analysis, the isomorphic conclusion no longer holds. This is because one more term appears in the policy maker's objective function. ${ }^{48}$ That is,

$$
\begin{aligned}
& E_{t} \sum_{i=0}^{\infty} \beta^{i} U_{t+i} \approx-\frac{1}{2}(1+\mu) u_{c} \bar{Y} \\
& E_{t} \sum_{i=0}^{\infty} \beta^{i}\left\{\begin{array}{l}
x_{t+i}{ }^{2}+\frac{\omega \theta^{2}}{(1-\omega)(1-\omega \beta)} \hat{\Pi}_{H, t+i}{ }^{2} \\
+\frac{2 \alpha}{(1+\mu)(1-\alpha)}\left(\hat{q}_{t+i}-\hat{q}_{t+i}{ }^{f}\right) \hat{\chi}_{1 t+i}
\end{array}\right\}+\text { ti. } . p .+o\left(\|\dot{\xi}\|^{3}\right)
\end{aligned}
$$

\footnotetext{
${ }^{47}$ In comparison with our work, they also ignore the preference shock, $\chi_{1 t}$.

${ }^{48}$ Kirsanova et al (2006) argue that this isomorphism holds even if they generalized Gali and Monacelli (2005) to include preference and international risk sharing shocks.
} 
Finally, we note that it can also be shown that the assumption of complete financial markets results in the real exchange rate gap moving proportionately with the output gap through the relationship, $\hat{q}_{t}-\hat{q}_{t}^{f}=(\sigma(1-\alpha) / \phi) x_{t}$. Applying this in our context we obtain

$$
\begin{aligned}
& E_{t} \sum_{i=0}^{\infty} \beta^{i} U_{t+i} \approx-\frac{1}{2}(1+\mu) u_{c} \bar{Y} \\
& E_{t} \sum_{i=0}^{\infty} \beta^{i}\left\{\begin{array}{l}
x_{t+i}^{2}+\frac{\omega \theta^{2}}{(1-\omega)(1-\omega \beta)} \\
+\frac{2 \alpha \sigma}{\phi(1+\mu)} x_{t+i} \hat{\chi}_{1 t+i}
\end{array}\right\}+\text { t.i.p. }+o\left(\|\ddot{\mid}\|^{\beta}\right)
\end{aligned}
$$

In this sense, Equation (3.52) shows that the real exchange rate need not explicitly enter the policy maker's objective function. It also implies that under complete asset markets, a flexible exchange rate regime is desirable even if the policy maker's objective function in a small open economy is not isomorphic to the closed economy case.

We can now see that by relaxing the strong conditions imposed by Gali and Monacelli (2002), as in (3.50), that the policy maker's objective function can no longer simply be written as quadratic terms in the output gap and inflation and a cross product term. It will involve more quadratic and cross terms. The first new quadratic term included in the policy maker's objective function is the real exchange rate. As mentioned above, the output gap moves proportionately with the real exchange rate gap only under the assumption of complete asset markets when the real exchange rate gap can be rewritten into the coefficient of the output gap. However, allowing for incomplete asset markets means that domestic households' consumption risks cannot be shared with their counterparts in the rest of the world. This can be seen in (3.13). It follows that the real exchange rate gap cannot be replaced by or incorporated within other gap terms and 
instead becomes a truly independent target for the policy maker's objective function. The same thing arises because of the assumption of incomplete pass through. Separate costs of adjustment in import prices imply the presence of an additional quadratic term representing the law of one price gap.

In addition to the quadratic terms, a more general analysis adds several cross product terms to the analysis that we denote by c.p.t. These involve the output gap, the real exchange rate gap, the law of one price gap, and net foreign assets. Moreover, the objective function also contains a number of terms that linearly combine the various gaps and exogenous shocks. Following Kirsanova et al (2006), these terms can be described as "linear in policy" denoted as l.i.p. In these cases, $\hat{Y}_{t}^{f}$ (or $\hat{q}_{t}^{f}$ ) depart from their efficient steady-state values when shocks occur and social welfare is changed if output levels or the real exchange rate departs its flexible price equilibrium. The effects of these "linear in policy" terms on social welfare are ambiguous, however, because the gaps sometimes move in the same direction as $\hat{Y}_{t}^{f}$ (or $\hat{q}_{t}^{f}$ ) and sometimes in the opposite direction, depending on the particular shocks that hit the economy. As Kirsanova et al (2006) emphasized, these "linear in policy" terms are important because they imply that in a small open economy it might not be optimal for policy to aim to reproduce the flexile price equilibrium. This point has also been noted by Benigno and Benigno (2003) among others.

While the cross product and linear policy terms all affect social welfare, the overall effect is ambiguous because the signs of these terms are not same and because different shocks have different effects on output gaps, the real exchange rate gaps, and the law of 
one price gaps. Thus the overall net effect of c.p.t. and li.p. on the social welfare might be quite small.

With this background, we rewrite equation (3.50) as

$$
\begin{aligned}
& E_{t} \sum_{i=0}^{\infty} \beta^{i} U_{t+i} \approx-\Omega E_{t} \sum_{i=0}^{\infty} \beta^{i}\left\{\begin{array}{l}
\Pi_{H, t+i}{ }^{2}+\lambda_{1} x_{t+i}{ }^{2}+\lambda_{2} Q g_{t+i}{ }^{2}+\lambda_{3} \hat{\Psi}_{t+i}{ }^{2} \\
+ \text { c.p.t. }+ \text { l.i.p. }
\end{array}\right\} \\
& + \text { t.i.p. }+o\left(\|\ddot{\xi}\|^{3}\right)
\end{aligned}
$$

where

$$
\begin{gathered}
\Omega=\frac{1}{2} \frac{\omega \theta^{2}(1+\mu)}{(1-\omega)(1-\omega \beta)} u_{c} \bar{Y}, \\
\lambda_{1}=(\mu+\sigma) \frac{(1-\omega)(1-\omega \beta)}{\omega \theta^{2}(1+\mu)}, \\
\lambda_{2}=(\sigma-1) \Theta^{2} \frac{(1-\omega)(1-\omega \beta)}{\omega \theta^{2}(1+\mu)}, \\
\lambda_{3}=(\sigma-1)\left(\frac{\alpha \eta}{1-\alpha}\right)^{2} \frac{(1-\omega)(1-\omega \beta)}{\omega \theta^{2}(1+\mu)} .
\end{gathered}
$$

The period loss function prior to including real money balances can then be written as

$$
\left.L_{1}=\operatorname{var} \Pi_{H, t}+\lambda_{1} \operatorname{var} x_{t}+\lambda_{2} Q g_{t}+\lambda_{3} \hat{\Psi}_{t}\right)+c . p . t .+ \text { li.p.t. }
$$

where var. stands for the variance operator.

\subsection{The Period Loss Function with the Real Balances}

We now introduce real balances into the analysis where for convenience, we write real balances as $m_{t}=M_{t} / P_{t}$. Following the same linearization procedure as we used before, $u\left(M_{t} / P_{t} ; \xi_{t}\right)$ can be expanded as 


$$
\begin{aligned}
& u\left(m_{t} ; \xi_{t}\right)=u(\bar{m} ; 1)+u_{m}(\bar{m} ; 1)\left(m_{t}-\bar{m}\right)+u_{\xi}(\bar{m} ; 1)\left(\xi_{t}-1\right)+\frac{1}{2} u_{m m}(\bar{m} ; 1)\left(m_{t}-\bar{m}\right)^{2} \\
& +u_{m \xi}(\bar{m} ; 1)\left(m_{t}-\bar{m}\right)\left(\xi_{t}-1\right)+\frac{1}{2} u_{\xi \xi}(\bar{m} ; 1)\left(\xi_{t}-1\right)^{2}+o\left(\|\xi\|^{3}\right)
\end{aligned}
$$

Using the formula (3.6), equation (3.55) can be written as

$$
\begin{aligned}
& u\left(m_{t} ; \xi_{t}\right)=u(\bar{m} ; 1)+u_{m} \bar{m}\left(\hat{m}_{t}+\frac{1}{2} \hat{m}^{2}\right)+u_{\xi}\left(\hat{\xi}_{t}+\frac{1}{2} \hat{\xi}_{t}^{2}\right) \\
& +\frac{1}{2} u_{m m} \bar{m}^{2}\left(\hat{m}_{t}+\frac{1}{2} \hat{m}^{2}\right)^{2} \\
& +u_{m \xi} \bar{m}\left(\hat{m}_{t}+\frac{1}{2} \hat{m}^{2}\right)\left(\hat{\xi}_{t}+\frac{1}{2} \hat{\xi}_{t}{ }^{2}\right)+\frac{1}{2} u_{\xi \xi}\left(\hat{\xi}_{t}+\frac{1}{2} \hat{\xi}_{t}^{2}\right)^{2}+o\left(\|\xi\|^{3}\right)
\end{aligned}
$$

where terms $u(\bar{m} ; 1), u_{\xi}$, and $u_{\xi \xi} / 2$ are independent of policy, and terms involved $\hat{m}_{t}{ }^{i}$ for $i \geq 3$ and $\hat{m}_{t}{ }^{2} \hat{\xi}_{t}$ can be ignored, thus we rewrite it as

$$
\begin{aligned}
& u\left(m_{t} ; \xi_{t}\right)=u_{m} \bar{m}\left(\hat{m}_{t}+\frac{1}{2} \hat{m}^{2}\right)+\frac{1}{2} u_{m m} \bar{m}^{2} \hat{m}_{t}^{2}+u_{m \xi} \bar{m} \hat{m}_{t} \hat{\xi}_{t}+t i \cdot p .+o\left(\|\xi\|^{3}\right) \\
& =u_{m} \bar{m}\left[\hat{m}_{t}+\frac{1}{2}\left(1+\frac{u_{m m}}{u_{m}} \bar{m}\right) \hat{m}_{t}{ }^{2}+\frac{u_{m \xi}}{u_{m}} \hat{m}_{t} \hat{\xi}_{t}\right]+t . i . p .+o\left(\|\xi\|^{3}\right)
\end{aligned}
$$

We may define $\varsigma^{-1}=-\frac{u_{m}}{u_{m m} \bar{m}}$, which is the intertemporal elasticity of substitution in real balance, therefore $\varsigma$ represents the coefficient of relative risk aversion, and $b_{3 t}=-\frac{u_{m \xi}}{u_{m m} \bar{m}} \bar{\xi}_{t}$. Applying this notation to (3.57) yields

$$
u\left(m_{t} ; \xi_{t}\right)=u_{m} \bar{m}\left[\hat{m}_{t}+\frac{1}{2}(1-\varsigma) \hat{m}_{t}^{2}+\varsigma_{3 t} \hat{m}_{t}\right]+t . i \cdot p \cdot+o\left(\|\xi\|^{3}\right)
$$


Next multiply and divide the right side of (3.58) by $u_{c} \bar{Y}$, then define $\varpi=u_{m} \bar{m} / u_{c} \bar{Y}$ (which measures the interest cost of real balances as a fraction of the value of individual consumption expenditure), to write (3.58) as

$$
u\left(m_{t} ; \xi_{t}\right)=u_{c} \bar{Y} \varpi\left[\hat{m}_{t}+\frac{1}{2}(1-\varsigma) \hat{m}_{t}^{2}+\varsigma b_{3_{t}} \hat{m}_{t}\right]+\text { t.i.p. }+o\left(\|\xi\|^{3}\right)
$$

Since the $\log$ deviation of real balances from its steady state, $\hat{m}_{t}$, is a stationary process and because $b_{3 t}=0$ under our specification of the representative household's utility function, the term involving $\hat{m}_{t}$ drops out of (3.59) to give us

$$
\begin{aligned}
& u\left(m_{t} ; \xi_{t}\right)=u_{c} \bar{Y} \varpi\left[\frac{1}{2}(1-\varsigma)\left(\hat{m}_{t}-\hat{m}_{t}^{f}\right)^{2}+(1-\varsigma)\left(\hat{m}_{t}-\hat{m}_{t}^{f}\right) \hat{m}_{t}^{f}\right] \\
& + \text { ti.p. }+o\left(\|\xi\|^{3}\right)
\end{aligned}
$$

From earlier thesis work, we derived the condition $\hat{m}_{t}=(1 / \varsigma)\left[\sigma \hat{C}-(\beta /(1-\beta)) \hat{R}_{t}-\hat{\chi}_{1 t}\right.$. Substituting (3.13) into it yields

$$
\hat{m}_{t}=\frac{\sigma}{\varsigma} \hat{Y}_{t}-\frac{\beta}{\varsigma(1-\beta)} \hat{R}_{t}-\frac{\sigma \Theta}{\varsigma} \hat{q}_{t}+\frac{\alpha \sigma \eta}{\varsigma(1-\alpha)} \hat{\Psi}_{t}-\frac{1}{\varsigma} \hat{\chi}_{1 t}+\frac{\alpha}{\zeta} \hat{\chi}_{2 t}
$$

where $\hat{R}_{t}$ is the $\log$ deviation of the gross nominal interest rate from its steady state value. The log deviation of the real balance prevailing under flexible prices and complete asset markets from its steady-state value, $\hat{m}_{t}^{f}$ can be defined as

$$
\hat{m}_{t}^{f}=\frac{\sigma}{\varsigma} \hat{Y}_{t}^{f}-\frac{\beta}{\varsigma(1-\beta)} \hat{R}_{t}^{f}-\frac{\sigma \Theta}{\varsigma} \hat{q}_{t}^{f}-\frac{1}{\varsigma} \hat{\chi}_{1 t} .
$$

Then subtracting (3.62) from (3.61) yields

$$
\hat{m}_{t}-\hat{m}_{t}^{f}=\frac{\sigma}{\zeta} x_{t}-\frac{\beta}{\zeta(1-\beta)} R g_{t}-\frac{\sigma \Theta}{\zeta} Q g_{t}+\frac{\alpha \sigma \eta}{\zeta(1-\alpha)} \hat{\Psi}_{t}+\frac{\alpha}{\zeta} \hat{\chi}_{2 t},
$$


where $R g_{t}=\hat{R}_{t}-\hat{R}_{t}^{f}$. Substitute (3.63) into (3.60) yields

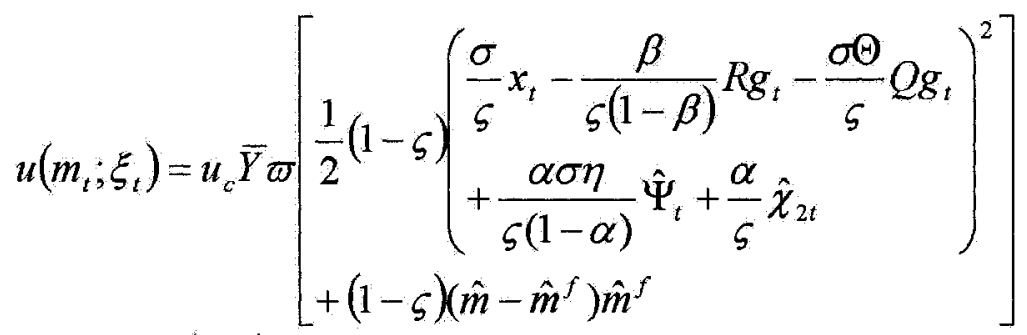

$$
\begin{aligned}
& + \text { t.i.p. }+o\left(\|\xi\|^{3}\right) \\
& =u_{c} \bar{Y} \varpi \frac{1}{2}(1-\varsigma)\left(\begin{array}{l}
\left(\frac{\sigma}{\varsigma}\right)^{2} x_{t}{ }^{2}+\left(\frac{\beta}{\varsigma(1-\beta)}\right)^{2} R g_{t}{ }^{2}+\left(\frac{\sigma \Theta}{\varsigma}\right)^{2} Q g_{t}{ }^{2} \\
+\left(\frac{\alpha \sigma \eta}{\varsigma(1-\alpha)}\right)^{2} \hat{\Psi}_{t}^{2}+c . p . t .+ \text { li.p.t. }
\end{array}\right) \\
& + \text { t.i.p. }+o\left(\|\xi\|^{3}\right)
\end{aligned}
$$

Note that because the utility function in (3.2) is additive rather than multiplicative, (3.64) can simply be combined with (3.50), to obtain ${ }^{49}$

$$
\begin{aligned}
& E_{t} \sum_{i=0}^{\infty} \beta^{i} U_{t+i} \approx-\frac{1}{2} u_{c} \bar{Y}
\end{aligned}
$$

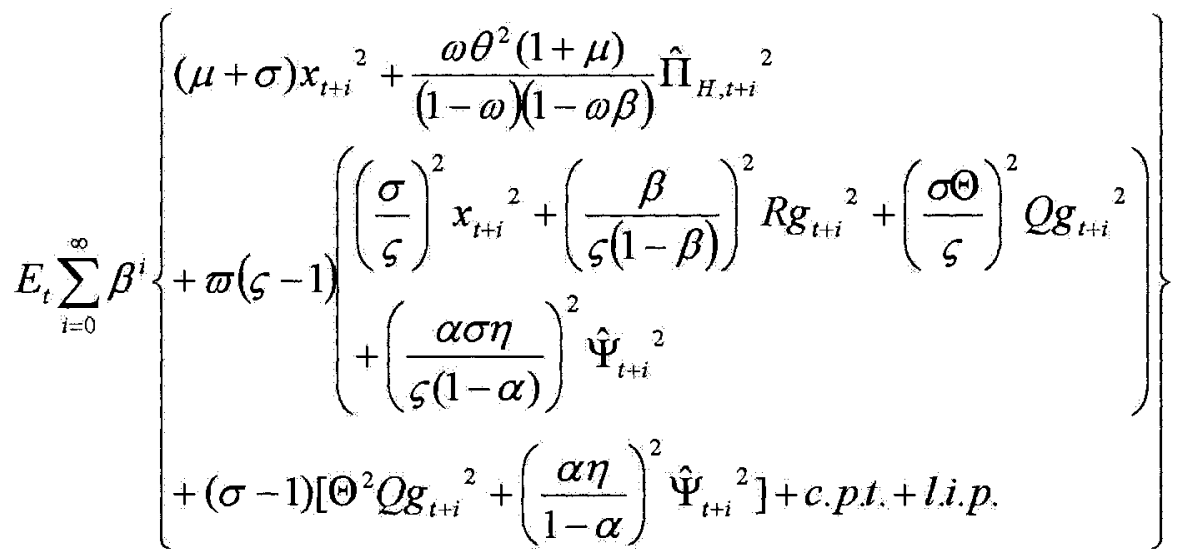

$$
\begin{aligned}
& +t i . p .+o\left(\|\ddot{\xi}\|^{3}\right)
\end{aligned}
$$

Collecting common terms gives

\footnotetext{
${ }^{49}$ Alternatively equation ( 3.60 ) can be directly combined with (3.50), then the variance of the real balance gap rather than the interest rate gap becomes an additional term in the welfare function.
} 


$$
\begin{aligned}
& E_{t} \sum_{i=0}^{\infty} \beta^{i} U_{t+i} \approx-\Omega E_{t} \sum_{i=0}^{\infty} \beta^{i}\left\{\begin{array}{l}
\hat{\Pi}_{H, t+i}{ }^{2}+\phi_{1} x_{t+i}{ }^{2}+\phi_{2} Q g_{t+i}{ }^{2} \\
+\phi_{3} \hat{\Psi}_{t+i}{ }^{2}+\phi_{4} R g_{t+i}{ }^{2}+\text { c.p.t.t. li. } . p .
\end{array}\right\} \\
& + \text { ti.p. }+o\left(\|\xi \dot{\xi}\|^{3}\right)
\end{aligned}
$$

where

$$
\begin{gathered}
\Omega=\frac{1}{2} \frac{\omega \theta^{2}(1+\mu)}{(1-\omega)(1-\omega \beta)} u_{c} \bar{Y} \\
\phi_{1}=\left(\mu+\sigma+\frac{\varpi \sigma^{2}(\zeta-1)}{\varsigma^{2}}\right) \frac{(1-\omega)(1-\omega \beta)}{\omega \theta^{2}(1+\mu)} \\
\phi_{2}=\left((\sigma-1) \Theta^{2}+\frac{\varpi \sigma^{2} \Theta^{2}(\zeta-1)}{\varsigma^{2}}\right) \frac{(1-\omega)(1-\omega \beta)}{\omega \theta^{2}(1+\mu)} \\
\phi_{3}=\left[(\sigma-1)\left(\frac{\alpha \eta}{1-\alpha}\right)^{2}+\varpi(\zeta-1)\left(\frac{\alpha \sigma \eta}{\zeta(1-\alpha)}\right)^{2}\right]\left(\frac{(1-\omega)(1-\omega \beta)}{\omega \theta^{2}(1+\mu)}\right. \\
\phi_{4}=\varpi(\varsigma-1)\left(\frac{\beta}{\varsigma(1-\beta)}\right)^{2} \frac{(1-\omega)(1-\omega \beta)}{\omega \theta^{2}(1+\mu)}
\end{gathered}
$$

The comprehensive period loss function that incorporates real money balances can then be written as

$$
\begin{aligned}
& L_{2}=\operatorname{var} \hat{\Pi}_{H, t}+\phi_{1} \operatorname{var} x_{t}+\phi_{2} \operatorname{var} Q g_{t} \\
& +\phi_{3} \operatorname{var} \hat{\Psi}_{t}+\phi_{4} \operatorname{var} R g_{t}+\text { c.p.t. }+ \text { li.p. }
\end{aligned}
$$

When we compare (3.67) with (3.54), we see that one additional term appears in the objective function once real money balances are incorporated into the analysis. ${ }^{50}$ The new term is the variance in the nominal interest rate gap, $\phi_{4} R_{t}{ }^{2}$. Intuitively, it arises because in our analysis, the level of real money balances is the residual endogenous

\footnotetext{
${ }^{50}$ This abstracts from the fact that some of the coefficients will also have changed.
} 
variable, determined by the nominal interest rate in combination with output and inflation. Hence given the real demand for money, any increased variation in the nominal interest rate will increase the variation in household's real balance holdings and so decrease its welfare level.

Before leaving this topic it is important to note that the standard period loss function used in the literature is usually assumed to be a weighted sum of only two arguments: the squared variances of inflation around its target level $\hat{\Pi}^{*}$ and the output gap, that is,

$$
L=\operatorname{var}\left(\hat{\Pi}_{H, t}-\hat{\Pi}^{*}\right)+a \operatorname{var} x_{t}
$$

where $\hat{\Pi}^{*}$ is often assumed to be zero, and $a$ takes a value somewhere between 0 and 1 . One such value given to a is 0.5 (see Svensson, 2000). However, when we use the calibrated parameter values that will be adopted for simulation purposes in the following chapter, the calibrated weight to be placed on the output gap from our utility function can be calculated to be approximately 0.0025 . While this weight may seem quite small, it is worth noting that finding of a small weight for the output gap in the loss function is not specific to our model, but rather typical of the weightings found in other studies where the loss function has been derived from the representative household's utility function. For example, using the calibrated parameter values adopted by Rotemberg and Woodford (1999), the weight that is placed on the output gap in their loss function is 0.00298 . Similarly, Gali and Monacelli (2002) find that their value for $a$ is equal to 0.0027 . 


\subsection{Concluding Remarks}

Following the methodology of Rotemberg and Woodford (1999) and Woodford (2003) we have derived in this chapter the policy maker's objective function in our small open economy by linearizing the representative household's utility function to the second order. Under the assumptions of incomplete asset markets and imperfect exchange rate pass-through the objective function even the real counterpart to our model will contains at least four key terms: the inflation rate, the output gap, the law of one price gap, and the net foreign asset position. In addition, once we account for the appearance of real balances in the household utility function, the nominal interest rate gap becomes the key fifth term. Finally, as we have seen, the objective function in a small open economy will be isomorphic to the closed economy case only under very special conditions that are not present in our model. Rather in our small open economy model, the loss function will differ considerably from its closed economy counterpart. 


\section{Chapter 4}

\section{Calibration and Simulation Analysis}

The solution to the model represented by the set of simultaneous equations in Chapter 2 is too complicated to work with analytically in a tractable way. Hence we follow the literature in setting up a quantitative dynamic stochastic general equilibrium model to evaluate alternative monetary policy rules based on the model's simulated results. To set up this model we use the calculating routines of DYNARE, a stochastic simulation program written to work with MATLAB. ${ }^{51}$ To that end we first calibrate the structural parameters of the model, then illustrate the working of the model with respect to one representative case. At that stage we add the welfare loss function developed in Chapter 3 as our basis for evaluating the simulated outcomes of the model for a number of different monetary policy rules.

\subsection{Calibration}

To use simulation techniques we needed to adopt specific values for the key parameters of the model and, to the extent that it was possible, these parameter values were chosen from the set of empirical estimates reported in the literature. In most cases, the parameter values used in our calibration are close to the mean of the available set of

\footnotetext{
${ }^{51}$ Dynare is available online and is the combined result of research efforts carried out at CEPREMAP by a group of individuals that have included: Jean-Pierre Laffargue, Raouf Boucekkine, Fabrice Collard and particularly, Michel Juillard.
} 
estimates, described in more detail below. The final parameter values used in our simulations appear in summary form in Table 4.1.

To begin, we adopt the value of 0.99 for the subjective discount factor, $\beta$. This is now a common choice for researchers and yields an annual value for the steady state real rate of interest of four percent. There is much less agreement on the other parameters of the model, however. If we start by describing the outcomes available for the parameters of the utility function we find, for example, that for coefficient on the consumption bundle, the literature has generated a variety of estimates of the intertemporal elasticity of substitution, $1 / \sigma$. Using U.S. data, Rotemberg and Woodford (1997) estimate $\sigma$ to be 0.16, whereas Casares (2001), using euro area data and a structural consumption equation, finds an estimate of $\sigma$ as 1.25 . In our analysis, we adopt the overall verdict given by Blanchard and Fischer in their standard text (1989) that the empirical literature as a whole yields an elasticity of substitution that is relatively small, lying at or below unity and so choose a calibration value for $\sigma$ of 1.2 (implying an elasticity of substitution of 0.83 ).

The second utility function parameter, $\varsigma$, relates to the utility value generated by real balances. Here we use the estimate by Chari et al (2002) of 2.5. In the analysis, $\varsigma$, is the inverse of the interest rate elasticity of money demand and a value of 2.5 implies a (negative) interest elasticity of $0.4{ }^{52}$ The third parameter of our utility function, $\mu$, is set equal to 3 . This corresponds to a labor supply elasticity of $1 / 3$ and lies roughly midway

\footnotetext{
${ }^{52} \mathrm{McC}$ allum and Nelson (1999) report the estimate of the interest elasticity of money demand of 0.15 (with a standard error of 0.018 ) using an instrumental variables approach based on US sample quarterly data from 1955:1-1996:4.
} 
between the low estimate of 0.05 found by Smets and Wouters (2000) and the higher estimate of 0.47 found Rotemberg and Woodford (1997).

We turn next to the elasticity of substitution among the differentiated goods in the aggregate consumption bundle corresponding to the markup in the model. Martins et al. (1996) estimate the average markup in the manufacturing sector to be around 1.2 for most OECD countries over the period 1980-92, while authors such as Morrison (1994) and Domowitz et al. (1988) suggest that a range between 1.2 and 1.7 may be plausible. Here we set the markup as 1.2 . This requires a value for $\theta$ equal to 6 .

In terms of the special coefficients that define our small open economy, we choose a calibration value for the elasticity of substitution between domestic goods and foreign goods, $\eta$, of 1.2 . This is roughly the mean value of the estimates of this parameter given in the literature. For example, Ambler et al. (2003, 2004) report the estimated value of $\eta$ to be 0.5962 based on Canadian data. This is close to the calibrated value of 0.6 used by McCallum and Nelson (2001). Kollmann (2002) also uses value of 0.6 for this parameter. He justifies this calibration as being approximately equal to the median value estimated by Hooper and Marquez (1995). On the other hand, more recent authors such as Chari et al. (2002) and Smets and Wouters (2002) set the elasticity of substitution between domestic and foreign goods at 1.5 and Choudhri et al. (2003) calibrate this parameter as $5^{53}$,

In a small open economy such as Canada, the import to GDP ratio is around 0.4 . We take this as our value for $\alpha$, the share of consumption devoted to the foreign goods. The degree of price stickiness, $\omega$, is set at 0.75 . Smets and Wouters (2002) estimated the

\footnotetext{
${ }^{53}$ Choudhri (2005) sets $\eta$ to be 2 instead of 5 .
} 
degree to which prices are indexed to past inflation and find $\delta \approx 0$, that is, find very little evidence of indexation. For this reason we adopt for our simulations the value $\delta=0.1$. The parameter on the import price adjustment cost function $\omega_{M}$ is set equal to 400 and $b$, which governs the size of the risk premium, is set equal to 0.01 .

There are four shocks in our model. As was the case for many of the previous parameters, estimates of the standard deviations of the innovations in these stochastic processes have varied widely in magnitude across the literature. For example, using quarterly HP-filtered data over the sample period 1963:1-2002:4, Gali and Monacelli (2005) fit an $A R(1)$ process to (log) labor productivity in Canada to find that the standard deviation of labor productivity shock $\sigma_{A}$ of 0.0071 and a persistence value for the shock, $\rho_{A}$, of 0.66 . Ambler et al. (2004) use quarterly Canadian data from 1981:3-2002:4 to report estimates of $\sigma_{A}=0.0204$, and $\rho_{A}, 0.8797$. Ireland (2001) reports estimates of $\sigma_{A}$ as 0.0159 and $\rho_{A}$ as 0.9203 using 1959-1979 U.S. data; and $\sigma_{A}$ as 0.0082 and $\rho_{A}$ as 0.9564 using 1979-1998 U.S. data. In our simulations we set the standard deviation of the productivity shock at $\sigma_{A}=0.0098$, and $\rho_{A}=0.9$. The aggregate demand (taste) shock is set as $\sigma_{1}=0.0351$, and $\rho_{1}=0.90 .{ }^{54}$ Similarly we set the parameters of our risk premium shock at $\sigma_{2}=0.005$, and $\rho_{2}=0.5$. Finally for the foreign output shock, we set

\footnotetext{
${ }^{54}$ Ireland (2001) obtains the estimates: $\sigma_{1}=0.0306$ and $\rho_{1}=0.9399$ using U.S. 1959-1979 data; and $\sigma_{1}=0.0351$ and $\rho_{1}=0.8920$ using U.S. 1979-1998 data.
} 
$\sigma_{y^{*}}$ to be 0.0060 , and $\rho_{y^{*}}$ to be $0.88 .{ }^{55}$ The final values chosen are summarized in Table 4.1 below.

Table 4.1 The Parameter Values Chosen to Calibrate the Model

\begin{tabular}{llll}
\hline Parameters & Values & Parameters & Values \\
\hline Utility & & Shocks & \\
$\beta$ & 0.99 & $\rho_{A}$ & 0.85 \\
$\sigma$ & 1.2 & $\rho_{1}$ & 0.90 \\
$\varsigma$ & 2.5 & $\rho_{2}$ & 0.50 \\
$\mu$ & 3.0 & $\rho_{y^{*}}$ & 0.85 \\
& & $\sigma_{A}$ & 0.0098 \\
Shares & & $\sigma_{1}$ & 0.0351 \\
$\alpha$ & 0.4 & $\sigma_{2}$ & 0.0050 \\
$b$ & 0.01 & $\sigma_{y^{*}}$ & 0.0070 \\
$\delta$ & 0.1 & Substitution Elasticities \\
$\omega$ & 0.75 & $\eta$ & 1.2 \\
$\omega_{M}$ & 400 & $\theta$ & 6 \\
\hline
\end{tabular}

In the simulations to be done below we are interested in comparing both the performance of the model's key variables in relation to each other and across time, and the aggregate welfare consequences of the outcomes under different sets of monetary policy rules. All rules involve setting the current interest rate in response to different performance indicators and with different intensities for the response coefficients. For comparative purposes, we will allow the monetary authority access to different information sets (described as different cases below) and consider the consequences for five different monetary policy rules, as summarized in Table 4.2 below.

\footnotetext{
${ }^{55}$ Ambler et al. (2004) report the value of $\sigma_{y^{*}}=0.0066$, and $\rho_{y^{*}}, 0.8280$. Gali and Monacelli (2005) use ( $\log$ ) U.S. 1963:1-2002:4 GDP data to estimate $\sigma_{y^{*}}$ and $\rho_{y^{*}}$, and find that $\sigma_{y^{*}}=0.0078$ and $\rho_{y *}=0.86$.
} 
Rule 1 is called for convenience the comprehensive rule (sometimes called the baseline rule). Traditionally the monetary authority in a closed economy is described as setting a short-term interest rate in response to changes in the inflation rate and the real GDP output gap. However as we have seen from the model, in a small open economy the real exchange rate will also be an important endogenous variable and it is natural to ask whether the central bank should react to the real exchange rate when setting its short-term interest rate. In addition, under the assumption of incomplete exchange rate pass-through we have a law of one price gap introduced into the model to which the monetary authority may respond. Hence our baseline monetary policy rule is defined to include both of these open economy gaps.

From our earlier derivations in Chapter 2, however, we know that the law of one price gap is a function of both the real exchange rate and the inflation rate, so that the weighting given to these separate effects may over-weigh the importance of the law of one price gap when the monetary authority reacts explicitly to both the real exchange rate and the law of one price gap. Hence we ask whether the comprehensive rule can be improved when the monetary authority responds only to the real exchange rate. This we call Rule 2, the real exchange rate rule. For comparative purposes, then, we replace the real exchange rate term in the money rule with the law of one price gap. This becomes Rule 3, the exchange rate pass-through monetary policy rule.

Even though the model describes a small open economy, it is of interest to ask what will happen if the monetary authority completely ignores its openness and thus uses neither the real exchange rate nor the law of one price gap in setting its operating rule. This gives our fourth rule, the Taylor rule. Rule 5 further supposes that the monetary 
policy authority chooses to drop the real output gap to focus exclusively on inflation. This we call simply Inflation Targeting. ${ }^{56}$

As shown earlier, the actual inflation rate that arises when all prices are perfectly flexible and asset prices are fully complete, $\hat{\Pi}_{H, t}{ }^{f}$, is not zero each period (even though its average rate is zero). Hence it is of interest to examine the consequences of having the monetary authority respond to the inflation rate gap $\left(\hat{\Pi}_{H, t}-\hat{\Pi}_{H, t}{ }^{f}\right)$ rather than just $\hat{\Pi}_{H, t}$ (where $\hat{\Pi}_{H, t}{ }^{f}$ is implicitly assumed to be zero). This means that the above set of five rules can be repeated using the inflation rate gap rather than simply the inflation rate. In addition, it has become of independent interest to ask what will happen if the monetary authority chooses to smooth the variation in the interest rate it sets and so allow policy to react to lagged interest rates. Here the smoothing response coefficient, $\phi_{r}$, is chosen to stabilize interest rates and so meet the criteria $0<\phi_{r} \leq 1$. The larger is this response coefficient, the more smoothing will be imparted to interest rate policy. It follows that each of the five rules can be modified by attaching interest rate smoothing.

It is important to recognize that while the domestic output rate of inflation and the consumer price rate of inflation in a closed economy are the same, they will differ in a small open economy model when the relative price of domestic and foreign goods

\footnotetext{
${ }^{56}$ In the literature, the expression "targeting variable $\pi_{t}$ " or "having a target level $\pi^{*}$ for a variable $\pi_{t}$ " has two meanings. The first meaning is to use all relevant available information to bring the target variable in line with the target, or precisely to minimize some loss function over expected future deviations of the target variable from the target level. As defined in Svensson (1999), "strict" inflation targeting refers to the situation where only inflation enters the loss function, while "flexible" inflation targeting allows other goal variables, such as the output gap. According to the second meaning, "targeting" or "targets" imply a particular information restriction for the instrument rule, namely, that the instrument must only depend on the gap between the target variable and the target level. Here we use the second meaning (see Rudebusch and Svensson, 1999). Note that inflation targeting is also described as a framework for monetary policy rather than a rule. Historically, New Zealand became the first country in 1990 to adopt formal inflation targeting. Since then an increasing number of countries have adopted it in one form or another.
} 
changes and particularly when import prices are sticky. It follows that policy outcomes will be different depending on which price index the monetary authority chooses to stabilize. For this reason we are interested in seeing what difference it makes when the two different measures of inflation are used to set monetary policy and to evaluate through the loss function which measure of inflation makes the better measure for the monetary authority. ${ }^{57}$ Using the CPI measure of inflation to replace the domestic output price inflation in each of the five rules above gives us a second set of five different policy rule combinations.

For the remainder of this chapter we follow one strand of the literature by adopting specific values for the response coefficients, in our case the values: $\phi_{\pi}=0.5$, $\phi_{x}=0.5, \phi_{q}=0.3, \phi_{\Psi}=0.3$, and $\phi_{r}=0.8 .^{58}$ This is done to allow our model and its predictions for monetary policy performance to be compared to some of the standard models presented in the literature (see Rotemberg and Woodford, 1999, and Adolfson, 2005). In terms of the first two values Taylor $(1993,1997)$ suggests that the value for the inflation response coefficient as 1.5 and the value for the response coefficient on the output gap of $0.5 .^{59}$ [Note that our specification of the inflation response coefficient as 0.5 is equivalent to that of Taylor since our nominal interest rate target in the first term

\footnotetext{
${ }^{57}$ Note that all inflation-targeting central banks have chosen to measure the rate of inflation by reference to some version of the consumer price index, often a version of the index that excludes certain volatile components in order to focus on "core' inflation (see Bernanke et al., 1999). On the other hand, Gali and Monacelli (2005) find that the welfare losses by using the domestic inflation-based Taylor rule and the CPI inflation-based Taylor rule, respectively, are very similar.

58 In the following chapter we use the welfare function to solve for optimal simple policy rules by endogenizing the reaction coefficients.

${ }^{59}$ The Taylor principle argues that the short-term interest rate should be adjusted more than one-for-one in response to increases in inflation. Otherwise, the real rate of interest implied will fall and hence generate rather than restrain inflation, leading the inflation rate to become unstable. However, Bullard and Mitra (2002) argue that while $\phi_{\pi}>1$ is sufficient to satisfy the Taylor principle, values of $\phi_{\pi}<1$ can restrain inflation if the monetary authority compensates by choosing a sufficiently large value of $\phi_{x}$ or $\phi_{q}$.
} 
has already been adjusted for expected inflation]. Next, even though Ambler et al. (2003) estimate a relatively low value of $\phi_{q}=0.0525$, we assign a somewhat higher value to ensure that the openness feature of the model is reflected quantitatively. Finally we note that when in our baseline rule we use both the exchange rate and law of one price gap, a smaller coefficient size was used for both to avoid over-weighing openness and inducing policy instability.

Table 4.2 Monetary Policy Rules

\begin{tabular}{lllllll}
\hline Rule & Policy Description & $\phi_{\pi}$ & $\phi_{x}$ & $\phi_{q}$ & $\phi_{\Psi}$ & $\phi_{r}$ \\
\hline 1 & Comprehensive rule (C) & 0.5 & 0.5 & $0.15^{*}$ & $0.15^{*}$ & 0 \\
2 & Real exchange rate rule (EX) & 0.5 & 0.5 & 0.3 & 0 & 0 \\
3 & Exchange rate pass-through (PT) & 0.5 & 0.5 & 0 & 0.3 & 0 \\
4 & Taylor rule (TR) & 0.5 & 0.5 & 0 & 0 & 0 \\
5 & Inflation rate targeting (IT) & 0.5 & 0 & 0 & 0 & 0 \\
\hline
\end{tabular}

* A smaller response coefficient is used to avoid the problem of policy overreaction.

\subsection{Impulse Responses of Key Variables to Shocks}

Before presenting our evaluation of the different policy rules, we illustrate the working of the simulation model for a representative case. Hence in Figures 4.1 and 4.2 we present the response over 40 quarters of some of the model's important endogenous variables to an impulse from two of the model's four shocks: a productivity shock and a risk premium shock ${ }^{60}$ The results are presented for Rule 1, our baseline policy rule with

\footnotetext{
${ }^{60}$ The illustrations for the other two shocks are similar to the productivity shock and the risk premium shock, thus are omitted here.
} 
interest-rate smoothing (when inflation is measured in terms of domestic output inflation rates). ${ }^{61}$

The productivity $\operatorname{shock} \varepsilon_{A t}$ affects output directly through $A_{t}$ in the production function. Figure 4.1 shows that the GDP rises immediately when the economy is hit by the positive productivity shock and this income effect, in turn, increases household consumption (but less than in proportion due to consumption smoothing). Note that under the comprehensive monetary policy rule, actual and flexible output both change in the same direction but with different magnitudes so that the output gap, measured as the log deviation between the two, changes. It is sticky prices in the model that produces this difference, here keeping actual output from increasing as much as it would under the perfectly flexible prices. Hence the output gap becomes negative. Given our parameter values, higher household income leads to a decrease in the supply of labor, a result that is often found in the literature. For example, using a long-run identifying assumption in his vector autoregression model, Gali (1999) found that a shock to productivity is also associated with an initial decline in work effort and Choudhri (2005) uses a similarly signed labor supply effect. Following the positive technology shock, aggregate supply rises more than aggregate demand so that prices tend to fall. Hence to stabilize prices and close the output gap, the interest rate is reduced. On the other side, the tendency for prices to fall promotes the export of goods. This affects the nominal exchange rate, now jumping to a higher value before falling back to its initial level, an overshooting of its final value. With domestic prices declining and the currency depreciating, the real exchange rate is driven up. Net foreign assets are first accumulated as exports exceed

${ }^{61}$ The impulse response functions vary somewhat when alternative policy rules are adopted by the monetary authority but have roughly the same overall form. 
imports, reaching at a maximum after about 10 quarters, then the buildup in assets is slowly decumulated, gradually returning to its steady state value. The change in the exchange rate also results in a change in import prices, however because of the constraint implied by quadratic adjustment costs, importers do not raise their prices in the same proportion as the exchange rate, therefore a positive law of price gap is created. Finally, the response of real money balances to the productivity shock is similar to output but smaller in scale. The rise in output and fall in interest rates increases the real demand for money. This slowly falls back to its starting value as all other variables return to their steady state values.

Figure 4.1 Impulse responses to a productivity shock under rule 1 in Case 1
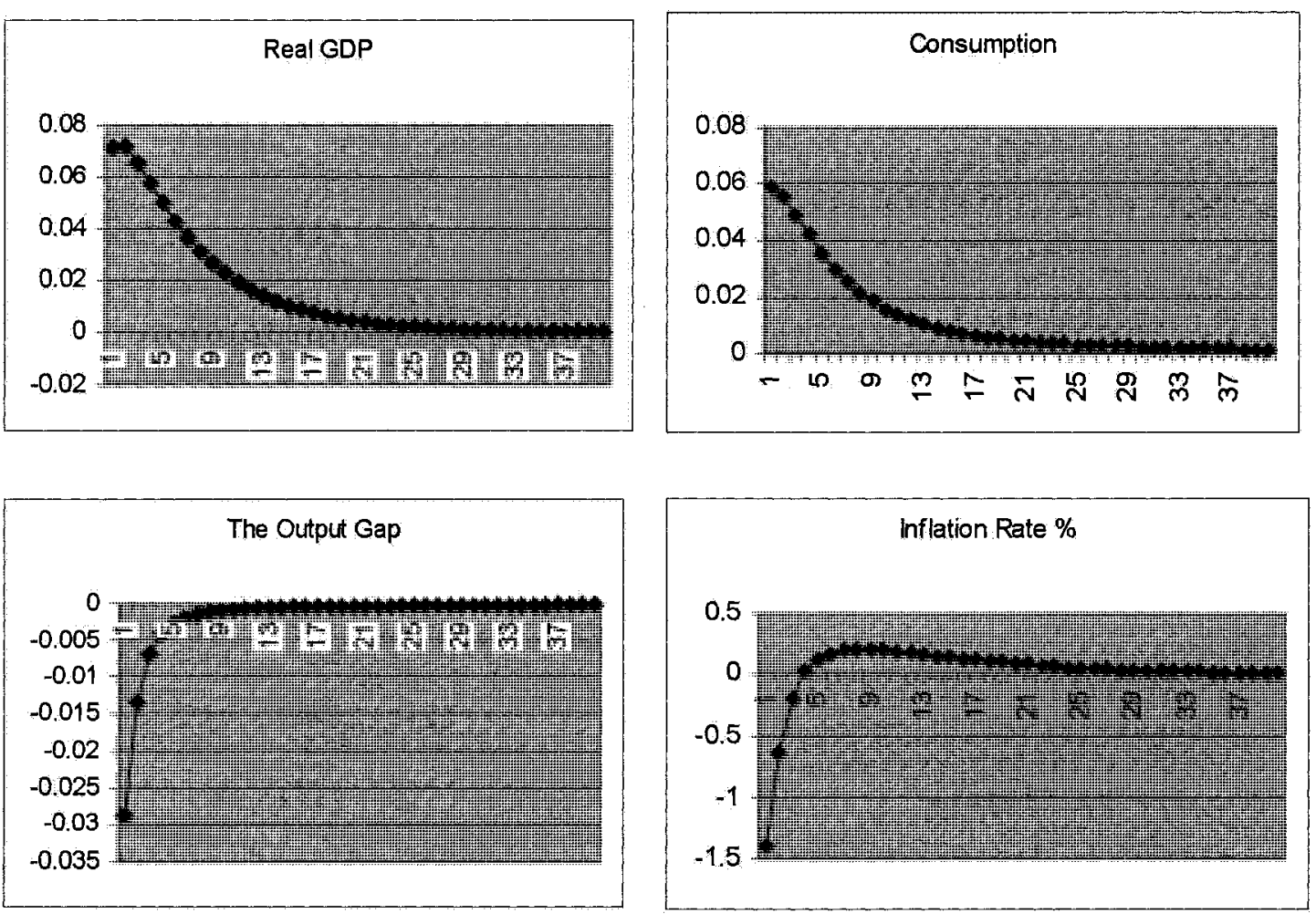

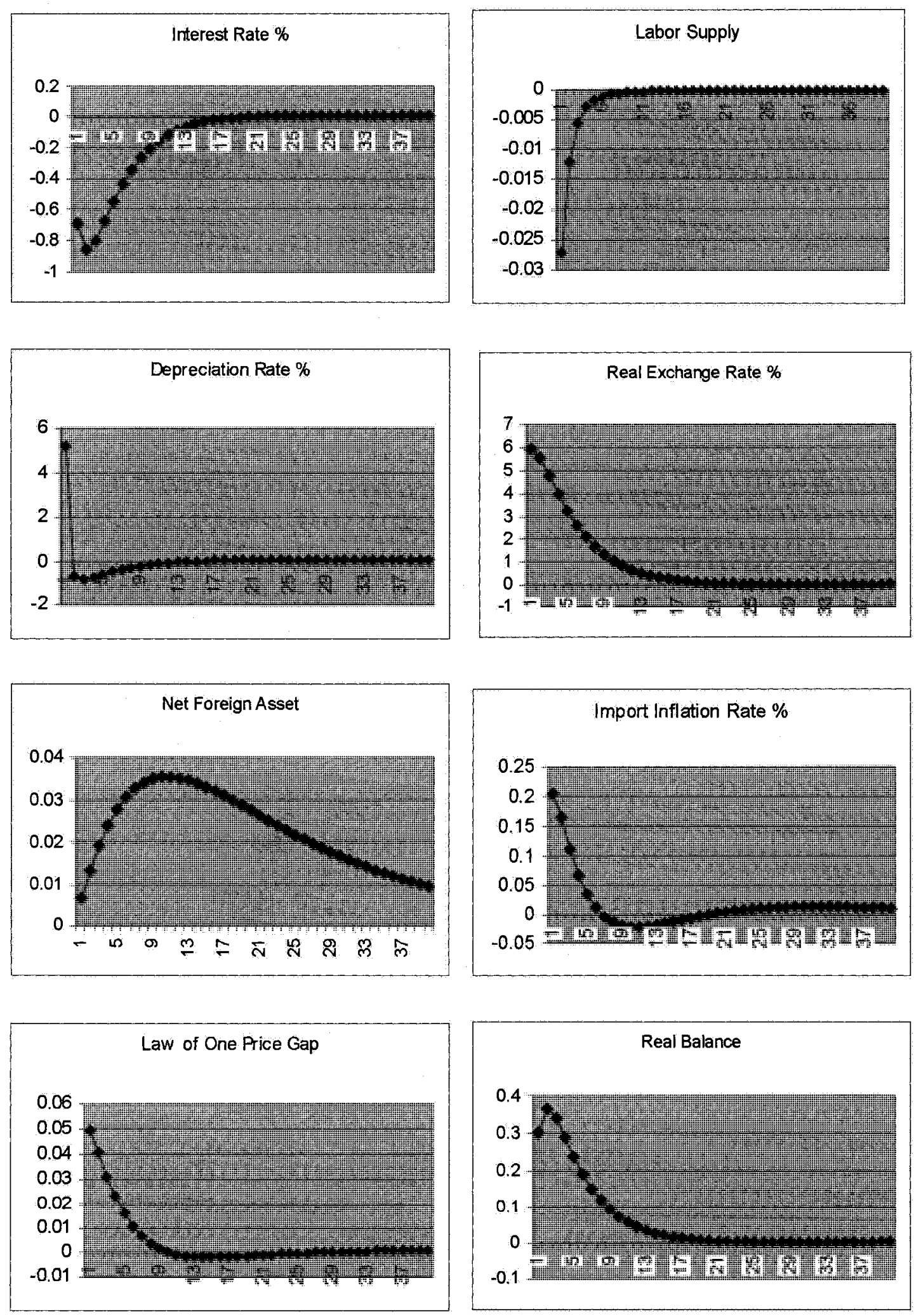
The dynamic impulse responses of the same set of endogenous variables to a risk premium shock are exhibited in Figure 4.3. In this case the risk premium shock is incorporated into the model via the uncovered interest parity condition. Here a positive risk premium shock pushes the domestic interest rate up and induces currency depreciation. This in turn boosts production and employment by increasing foreign demand for domestic output. In our analysis the foreign price is exogenous so that a positive law of one price gap is created as the currency depreciates. Hence with rising interest rates and both domestic and import prices rising, households do not increase consumption, instead choosing to accumulate net foreign assets.

Figure 4.2 Impulse responses to a risk premium shock under rule 1 in case 1
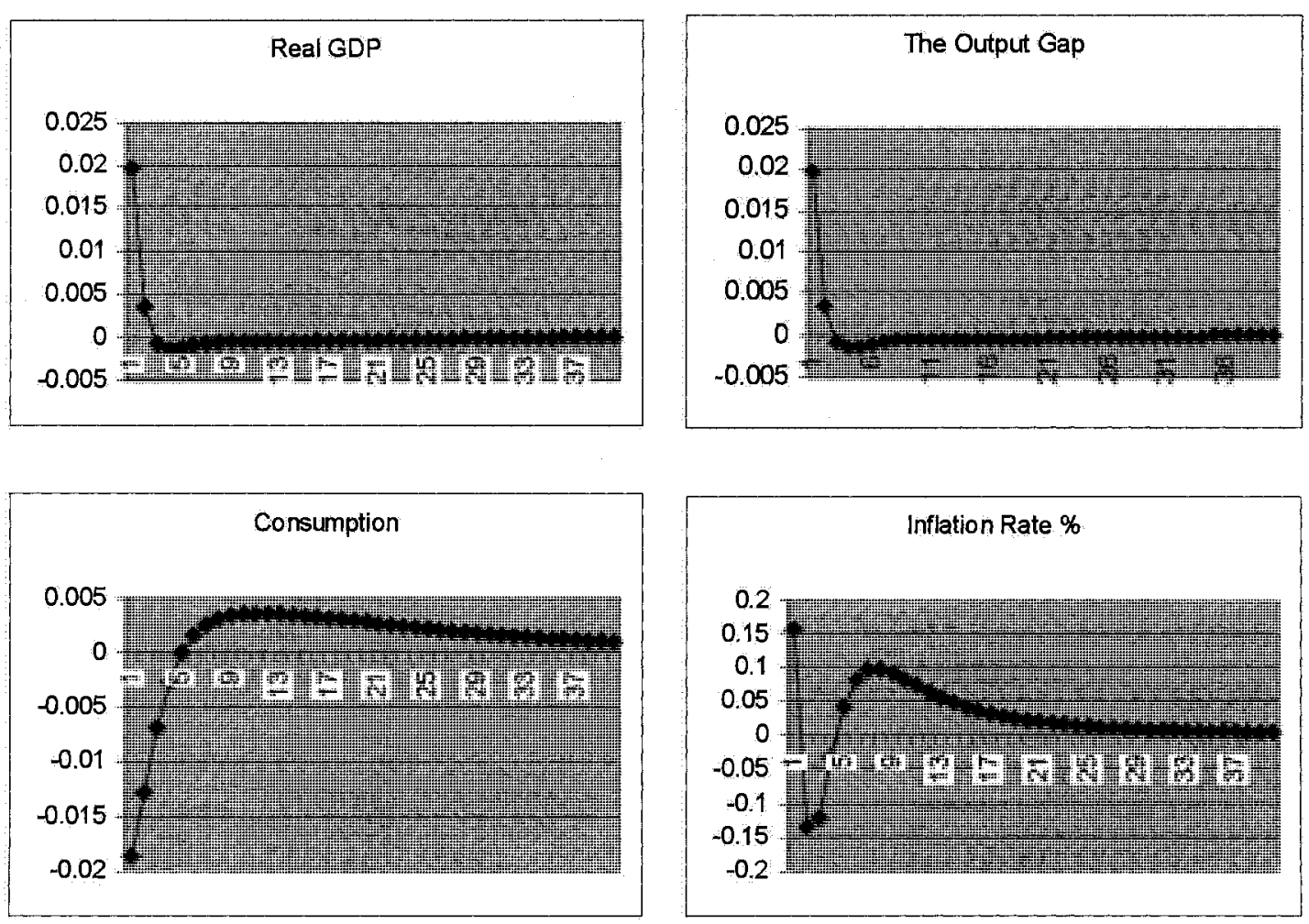

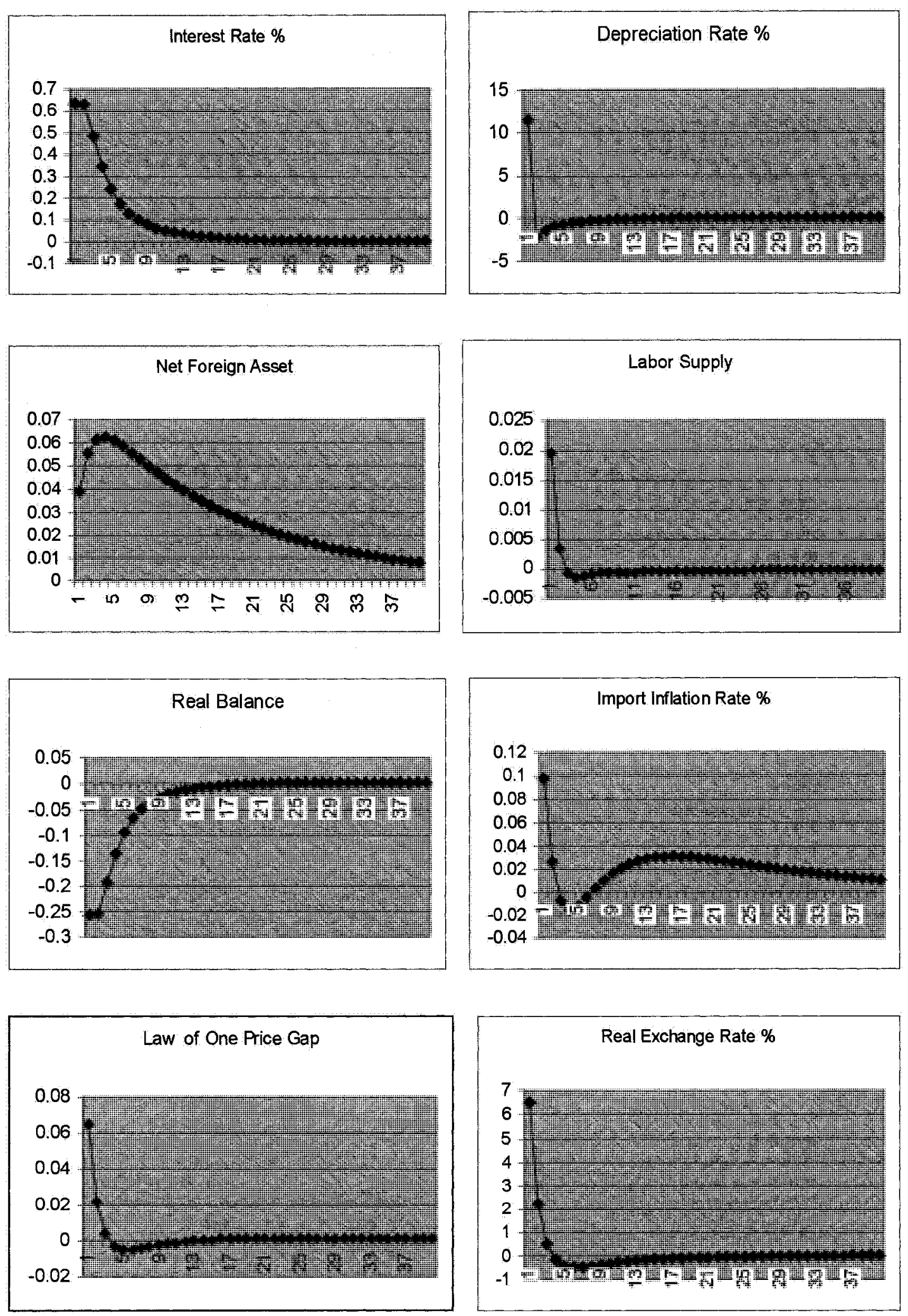


\subsection{The Welfare Consequences of Alternative Policy Rules: Contemporaneous}

\section{Response}

In this section we use the dynamic simulation results to evaluate the welfare implications of our five alternative monetary policy rules for the loss function derived as equation (3.67) of Chapter 3. The question that we are most interested in answering is how the different monetary rules perform relative to each other when the information set available to the monetary authority changes. More explicitly, in the cases considered below, the monetary authority is assumed to possess less and less accurate knowledge of the key gaps facing the economy, while the public is still assumed to have full information on the variables need to make their individually optimal decisions. Hence in the sections below we consider four different information regimes, with each successive regime incorporating less and less relevant information. In each case we ask how the information available to the monetary authority affects the ranking of the alternative monetary rules in relation to the model's set of shocks. In such a manner we isolate the monetary policy rule that in each case minimizes the loss function conditional on the specific information available to a central bank when making its decision on the nominal interest rate. We begin by considering the case where the central bank has sufficient information to able to target accurately the relevant efficiency gaps arising from the inability of private agents to react costlessly to current shocks. Then we consider how the desirability of each rule changes as we subtract information on the nature of the shocks hitting the model and the monetary authority is forced to use less accurate information in the implementing of its policy rule.

\section{Case 1: Full information}


In Case 1, we assume that the central bank is fully knowledgeable in the sense that it has access to all the information needed to observe or derive the contemporary gaps that arise between the flexible and sticky price versions of each targeted policy variable. Hence given this information, the monetary authority knows or can calculate what the interest rate would be in the absence of sticky prices. Traditionally, this has been called the Wicksellian 'natural' rate of interest, $i_{w_{s}}$. The log deviation of the gross Wicksellian interest rate, $R_{w, t} \equiv 1+i_{w, t}$, from its steady state level, $\bar{R}=1+\bar{i}$ has already appeared in our analysis as $\hat{r}_{H, t}{ }^{f}$ and hence this will be used as the first term in the money rule in equation (2.100) above. Similarly, with full information the monetary authority will also target an inflation rate that would prevail in the absence of price rigidities $\hat{\Pi}_{H, t}{ }^{f}$, and the output level that would arise under flexible prices, $\hat{Y}_{t}^{f}$. Finally, the monetary authority would also have the information needed to determine the real exchange rate, $\hat{q}_{t}^{f}$, that would arise in the absence of price rigidities and conditional on world output. Thus under the full information assumption, the interest rate reaction function that will frame our discussion of monetary policy can be written as

$$
\hat{R}_{t}=\left(1-\phi_{r}\right)\left[\begin{array}{l}
\hat{r}_{H, t}^{f}+\hat{\Pi}_{H, t}+\phi_{\pi}\left(\hat{\Pi}_{H, t}-\hat{\Pi}_{H, t}^{f}\right)+\phi_{x}\left(\hat{Y}_{t}-\hat{Y}_{t}^{f}\right) \\
+\phi_{q}\left(\hat{q}_{t}-\hat{q}_{t}^{f}\right)+\phi_{\Psi} \hat{\Psi}_{t}
\end{array}\right]+\phi_{r} \hat{R}_{t-1} .
$$

where the variables, $\hat{r}_{H, t}{ }^{f}, \hat{Y}_{t}^{f}$, and $\hat{q}_{t}^{f}$, are defined in terms of the model in Chapter 2 (see equations (2.91), (2.86), and (2.98)) and the values of parameters $\phi_{\pi}, \phi_{f}, \phi_{x}, \phi_{q}$, and $\phi_{\Psi}$ as given in Table 4.2. By assumption we have $\hat{\Psi}_{t}^{f}=0$. Similarly equation (2.101) can be written as 


$$
\hat{R}_{t}=\left(1-\phi_{r}\right)\left[\begin{array}{l}
\hat{r}_{t}^{f}+\hat{\Pi}_{t}+\phi_{\pi}\left(\hat{\Pi}_{t}-\hat{\Pi}_{t}^{f}\right)+\phi_{x}\left(\hat{Y}_{t}-\hat{Y}_{t}^{f}\right) \\
+\phi_{q}\left(\hat{q}_{t}-\hat{q}_{t}^{f}\right)+\phi_{\Psi} \hat{\Psi}_{t}
\end{array}\right]+\phi_{r} \hat{R}_{t-1} .
$$

where $\hat{r}_{t}^{f}$ is defined in terms of the model in Chapter 2 (see equation (2.94)).

We turn then to evaluate the relative performance of the alternative policy rules in terms of the welfare loss function derived in Chapter 3 from the household's utility function. The policy rules we consider and the values of the response coefficients are set out in Table 4.2. Once again, Case 1 assumes that the monetary authority has sufficient information to determine the flexible price values of the model's endogenous variables so that it is able to target monetary policy at the gap between these values and their actual sticky price outcomes.

Tables 4.3 through Table 4.10 present the welfare outcomes for our five alternative policy rules under Case 1 involving full current information. Each row of the table presents first the standard deviations for each of the key inefficiency gaps in the model followed by the calculated welfare loss associated with that particular monetary policy rule. A scanning of the data presented in Table 4.3 reveals the following. First of all, the performance of each money rule is different in virtually all gap dimensions. Second, unlike closed economy or even small open economy models with only one reason for having prices depart from their efficient flexible price level (say, for example, due to Calvo pricing), knowledge of the Wicksellian interest rate does not allow policy to replicate the flexible price outcome. Hence while policy choices can reduce the welfare loss it cannot drive that loss to zero. Third, the first three rules that involve the exchange rate in one form or another, all generate smaller variations in the real exchange rate and the law of one price gap, but induce larger fluctuations in inflation, the output gap and the 
interest rate, thus all result in welfare losses that are greater than the losses arising under rules that omit some response to the exchange rate or law of one price gap. By contrast, even though the Taylor rule causes larger fluctuations in the real exchange rate and law of one price gap, it does generate the smallest variation in inflation and the output gap among all five rules, therefore resulting in the smallest welfare loss. ${ }^{62}$ Finally, compared to the Taylor rule, inflation-targeting not only results in a larger deviation in the output gap, but also a larger deviation in inflation, resulting in a larger overall welfare loss. This implies that the output gap is an important policy targeting variable.

Table 4.3

\section{Case 1: Simple rules with domestic output prices}

\begin{tabular}{l|l|l|l|l|l|l}
\hline \multirow{2}{*}{$\begin{array}{l}\text { Policy } \\
\text { Rule }\end{array}$} & \multicolumn{5}{|c|}{ Standard Deviation } & \multirow{2}{*}{ Welfare Loss } \\
\cline { 2 - 6 } & $\hat{\Pi}_{H, t}$ & $x_{t}$ & $R_{H g, t}$ & $q_{g, t}$ & $\hat{\Psi}_{t}$ & \\
\hline 1 (C) & 0.0191 & 0.0310 & 0.0281 & 0.0767 & 0.1695 & $4.4021 \mathrm{e}-004$ \\
\hline 2 (EX) & 0.0156 & 0.0245 & 0.0288 & 0.0841 & 0.1933 & $3.2225 \mathrm{e}-004$ \\
\hline 3 (PT) & 0.0241 & 0.0372 & 0.0279 & 0.0721 & 0.1516 & $6.5572 \mathrm{e}-004$ \\
\hline 4 (TR) & 0.0096 & 0.0242 & 0.0212 & 0.1109 & 0.2392 & $1.3969 \mathrm{e}-004$ \\
\hline 5 (IT) & 0.0124 & 0.0328 & 0.0186 & 0.1169 & 0.2336 & $1.9331 \mathrm{e}-004$ \\
\hline
\end{tabular}

* where $R_{H g, t}=\hat{R}_{t}-\hat{r}_{H, t}{ }^{f}$, and $q_{g, t}=\hat{q}_{t}-\hat{q}_{t}^{f}$

Table 4.4 illustrates how the standard deviations of the endogenous gaps in the model change when the policy maker adds an interest-rate smoothing term to its policy rule. Here, surprisingly, the standard deviation of the interest rate remains roughly the same and in some cases is larger than it would have been under non-interest-rate smoothing policy rules. Moreover, the addition of the interest-rate smoothing term to the

\footnotetext{
${ }^{62}$ Note that this ranking is not independent of the size of the response coefficients chosen for the analysis. The reader is cautioned to defer judgment on the optimal rule until Chapter 5 where response coefficients are endogenized.
} 
policy rule also generates larger standard deviations among the other targeted variables. Compared to the first three rules, the change in the standard deviations of targeting variables under last two are very significant. For example, the standard deviations of inflation under rule 4 are increased from 0.0096 to 0.0202 , and the standard deviations of the output gap are increased from 0.0242 to 0.0399 . The following two salient features, therefore, can be summarized from Table 4.4. First, the rules that involve interest rate smoothing now result in standard deviations and welfare losses that are in each case larger than under the corresponding money rule without interest rate smoothing. Second, the relative pattern of rule performance is completely different from that appearing in Table 4.3. The first two rules that involve the exchange rate now result in smaller variations in the key efficiency gaps and hence result in lower welfare losses. The rules that do not involve the exchange rate tend to produce larger welfare losses.

\section{Table 4.4}

\section{Case 1: Simple rules with interest rate smoothing}

\begin{tabular}{l|l|l|l|l|l|l}
\hline \multirow{2}{*}{$\begin{array}{l}\text { Policy } \\
\text { Rule }\end{array}$} & \multicolumn{5}{|c|}{ Standard Deviation } & Welfare Loss \\
\cline { 2 - 6 } & $\hat{\Pi}_{H, t}$ & $x_{t}$ & $R_{H g, t}$ & $q_{g, t}$ & $\hat{\Psi}_{t}$ & \\
\hline $1(\mathbf{C})$ & 0.0193 & 0.0396 & 0.0280 & 0.0961 & 0.1917 & $4.5000 \mathrm{e}-004$ \\
\hline $2(\mathbf{E X})$ & 0.0161 & 0.0358 & 0.0283 & 0.1049 & 0.2164 & $3.3855 \mathrm{e}-004$ \\
\hline $\mathbf{3}(\mathbf{P T})$ & 0.0236 & 0.0438 & 0.0278 & 0.0903 & 0.1732 & $6.3366 \mathrm{e}-004$ \\
\hline $4(\mathbf{T R})$ & 0.0202 & 0.0399 & 0.0311 & 0.1300 & 0.2602 & $5.0534 \mathrm{e}-004$ \\
\hline $5(\mathbf{I T})$ & 0.0250 & 0.0486 & 0.0361 & 0.1348 & 0.2555 & $7.5391 \mathrm{e}-004$ \\
\hline
\end{tabular}

In Table 4.3 and 4.4 we have assumed that the monetary authority targets a zero rate for the inflation rate of domestic output (implicitly assuming that the fully flexible price inflation rate would be zero). Hence the monetary authority responds only to the 
variation in the inflation rate and other variable gaps. Now we relax this assumption and allow the monetary authority to respond to the inflation rate gap rather than the rate itself. These comparative performance results are summarized in Tables 4.5 and 4.6 (where 4.6 adopts interest rate smoothing). Roughly speaking, the general pattern of policy performance is somewhat different from the case in which the monetary authority responds only to the inflation rate alone (see Tables 4.3 and 4.4). Three features are worth mentioning. First, policy response to the gap does not behave as might have been expected. Not only is variation in the inflation rate larger, but the rule also results in larger deviations for each of the other variables, especially the output gap and the interest rate gap, resulting in a larger welfare loss. For instance, the smallest welfare loss when the monetary authority responds to the inflation gap is $6.3034 \mathrm{e}-004$, which is 3.5 times larger than that arising in the case in which the monetary authority responds to the inflation rate, that is, $1.3969 \mathrm{e}-004$. Second, a consequence of larger variations in the inflation rate gap and output gap is that the performances of the Taylor and inflation targeting rules are now worse than the first two rules involving the exchange rate. This is similar to the outcome when the policy maker responds to the inflation rate under interest-rate smoothing (in Table 4.4) but opposite to when the policy maker simply responds to the inflation rate without the interest-rate smoothing (see Table 4.3). Finally, comparing Table 4.5 with Table 4.6, we see that the introduction of interest rate smoothing now does improve the performance of virtually all policy rules. However, compared to Table 4.3 where the policy maker simply uses the inflation rate target (without smoothing) the result is still inferior. 
Table 4.5

Case 1: Inflation gaps measured in domestic output prices

\begin{tabular}{l|l|l|l|l|l|l}
\hline \multirow{2}{*}{$\begin{array}{l}\text { Policy } \\
\text { Rule }\end{array}$} & \multicolumn{5}{|c|}{ Standard Deviation } & \multirow{2}{*}{ Welfare Loss } \\
\cline { 2 - 6 } & $\hat{\Pi}_{H, t}$ & $x_{t}$ & $R_{H g, t}$ & $q_{g, t}$ & $\hat{\Psi}_{t}$ & \\
\hline $\mathbf{1}(\mathbf{C})$ & 0.0209 & 0.0515 & 0.0456 & 0.0723 & 0.1642 & $6.3034 \mathrm{e}-004$ \\
\hline $\mathbf{2 ( E X )}$ & 0.0211 & 0.0454 & 0.0499 & 0.0807 & 0.1945 & $6.7465 \mathrm{e}-004$ \\
\hline 3 (PT) & 0.0247 & 0.0575 & 0.0434 & 0.0684 & 0.1425 & $7.8731 \mathrm{e}-004$ \\
\hline $\mathbf{4}$ (TR) & 0.0284 & 0.0453 & 0.0592 & 0.1113 & 0.2542 & 0.0011 \\
\hline 5 (IT) & 0.0261 & 0.0602 & 0.0683 & 0.1173 & 0.2469 & 0.0011 \\
\hline
\end{tabular}

Table 4.6

Case 1: Domestic inflation gaps with interest-rate smoothing

\begin{tabular}{l|l|l|l|l|l|l}
\hline \multirow{2}{*}{$\begin{array}{l}\text { Policy } \\
\text { Rule }\end{array}$} & \multicolumn{5}{|c|}{ Standard Deviation } & Welfare loss \\
\cline { 2 - 6 } & $\hat{\Pi}_{H, t}$ & $x_{t}$ & $R_{H g, t}$ & $q_{g, t}$ & $\hat{\Psi}_{t}$ & \\
\hline $\mathbf{1}(\mathbf{C})$ & 0.0208 & 0.0465 & 0.0281 & 0.0879 & 0.1788 & $5.1156 \mathrm{e}-004$ \\
\hline 2 (EX) & 0.0185 & 0.0407 & 0.0296 & 0.0964 & 0.2058 & $4.2861 \mathrm{e}-004$ \\
\hline 3 (PT) & 0.0250 & 0.0520 & 0.0275 & 0.0832 & 0.1592 & $7.0167 \mathrm{e}-004$ \\
\hline 4 (TR) & 0.0218 & 0.0407 & 0.0321 & 0.1206 & 0.2511 & $5.7762 \mathrm{e}-004$ \\
\hline 5 (IT) & 0.0236 & 0.0512 & 0.0346 & 0.1221 & 0.2374 & $6.7600 \mathrm{e}-004$ \\
\hline
\end{tabular}

We turn next to the question of what difference it makes whether the monetary authority utilizes domestic output prices or consumer prices in its interest rate setting rule. Hence we replicate the twenty cases (rules) reported in Tables 4.3 through 4.6 for the use by the monetary authority of CPI inflation (in Tables 4.7 and 4.8) and the CPI inflation gap (in Tables 4.9 and 4.10). By examining the results set out below, several commonalities can be observed: 
First, of the five rules in Table 4.7 where the monetary authority targets the inflation rate (without smoothing) the Taylor rule again results in the smallest welfare loss. This is consistent with the best policy finding in Table 4.3 where the policy maker responded to the inflation rated measured in terms of domestic output prices.

Second, compared to Table 4.3 one can see that the standard deviations in the real exchange rate and the law of one price gap in Table 4.7 are smaller, while the deviations in the inflation, the output gap and the interest rate are larger, ending up with a larger overall welfare loss. In some sense this should not be surprising. That is, the CPI measure of inflation already incorporates part of the direct effect of an exchange rate change via its effect on import prices, even though the degree of exchange rate pass-through is incomplete in our model due to the import prices stickiness. By contrast, the use of domestic output price inflation does not include this direct effect, only the indirect effect that is transferred through expenditure switching effect on the aggregate demand. Hence we should expect smaller variations in the real exchange rate and the law of one price under consumer price measure of inflation.

Third, if the policy outcomes across monetary rules when responding to the CPI inflation rate (see Tables 4.7 and 4.8) are compared to when the monetary authority responds to the CPI inflation gap (see Tables 4.9 and 4.10) it is typically the gap that results in better performance. This is the exact opposite of our findings when the measure of inflation rate and inflation gap involved only domestic output prices.

Finally, if we compare the results in Table 4.8 with those in Table 4.7 and the results in Table 4.10 with those in Table 4.9, we see that interest rate smoothing does not enhance best policy performance even though policy performance is improved a bit under 
the first three rules that involve the exchange rate. This coincides to some extent with our findings for the use of domestic output price (as found in Tables 4.4).

Table 4.7

Case 1: The monetary authority responds to CPI inflation rates

\begin{tabular}{l|l|l|l|l|l|l}
\hline \multirow{2}{*}{ Policy Rule } & \multicolumn{5}{|c|}{ Standard Deviation } & \multirow{2}{*}{ Welfare loss } \\
\cline { 2 - 6 } & $\hat{\Pi}_{H, t}$ & $x_{t}$ & $R_{g, t}{ }^{*}$ & $q_{g, t}$ & $\hat{\Psi}_{t}$ & \\
\hline $\mathbf{1}(\mathbf{C})$ & 0.0288 & 0.0435 & 0.0328 & 0.0669 & 0.1368 & $9.3149 \mathrm{e}-004$ \\
\hline $\mathbf{2}$ (EX) & 0.0241 & 0.0359 & 0.0336 & 0.0708 & 0.1571 & $6.8661 \mathrm{e}-004$ \\
\hline 3 (PT) & 0.0333 & 0.0504 & 0.0318 & 0.0655 & 0.1216 & 0.0012 \\
\hline 4 (TR) & 0.0194 & 0.0344 & 0.0319 & 0.0946 & 0.1859 & $4.7335 \mathrm{e}-004$ \\
\hline 5 (IT) & 0.0351 & 0.5250 & 0.0335 & 0.1040 & 0.1669 & 0.0013 \\
\hline
\end{tabular}

* Note that $R_{g, t}=\hat{R}_{t}-\hat{r}_{t}^{f}$.

Table 4.8

Case 1: Inflation measured in terms of CPI and interest rate smoothing

\begin{tabular}{l|l|l|l|l|l|l}
\hline \multirow{2}{*}{ Policy Rule } & \multicolumn{5}{|c|}{ Standard Deviation } & \multirow{2}{*}{ Welfare loss } \\
\cline { 2 - 6 } & $\hat{\Pi}_{H, t}$ & $x_{t}$ & $R_{g, t}$ & $q_{g, t}$ & $\hat{\Psi}_{t}$ & \\
\hline $\mathbf{1}$ (C) & 0.0268 & 0.0476 & 0.0331 & 0.0874 & 0.1634 & $8.2403 \mathrm{e}-004$ \\
\hline $\mathbf{2}$ (EX) & 0.0221 & 0.0422 & 0.0337 & 0.0934 & 0.1843 & $5.9727 \mathrm{e}-004$ \\
\hline 3 (PT) & 0.0311 & 0.0528 & 0.0323 & 0.0842 & 0.1472 & 0.0011 \\
\hline 4 (TR) & 0.0242 & 0.0440 & 0.0402 & 0.1136 & 0.2141 & $7.3888 \mathrm{e}-004$ \\
\hline 5 (IT) & 0.0359 & 0.0580 & 0.0457 & 0.1183 & 0.1989 & 0.0015 \\
\hline
\end{tabular}

Table 4.9

Case 1: Inflation gaps measured in CPI

\begin{tabular}{l|l|l|l|l|l|l}
\hline Policy Rule & \multicolumn{5}{|c|}{ Standard Deviation } & Welfare loss \\
\cline { 2 - 6 } & $\hat{\Pi}_{H, t}$ & $x_{t}$ & $R_{g, t}$ & $q_{g, t}$ & $\hat{\Psi}_{t}$ & \\
\hline $1(\mathbf{C})$ & 0.0281 & 0.0547 & 0.0308 & 0.0641 & 0.1327 & $8.8304 \mathrm{e}-004$ \\
\hline
\end{tabular}




\begin{tabular}{l|l|l|l|l|l|l}
\hline 2 (EX) & 0.0240 & 0.0469 & 0.0326 & 0.0678 & 0.1570 & $6.7818 \mathrm{e}-004$ \\
\hline 3 (PT) & 0.0327 & 0.0617 & 0.0299 & 0.0639 & 0.1152 & 0.0012 \\
\hline 4 (TR) & 0.0171 & 0.0448 & 0.0293 & 0.0912 & 0.1921 & $3.7752 \mathrm{e}-004$ \\
\hline 5 (IT) & 0.0258 & 0.0650 & 0.3220 & 0.0991 & 0.1683 & $7.7156 \mathrm{e}-004$ \\
\hline
\end{tabular}

Table 4.10

Case 1: CPI inflation gaps and interest rate smoothing

\begin{tabular}{l|l|l|l|l|l|l}
\hline \multirow{2}{*}{ Policy Rule } & \multicolumn{5}{|c|}{ Standard Deviation } & \multirow{2}{*}{ Welfare loss } \\
\cline { 2 - 6 } & $\hat{\Pi}_{H, t}$ & $x_{t}$ & $R_{g, t}$ & $q_{g, t}$ & $\hat{\Psi}_{t}$ & \\
\hline $1(\mathbf{C})$ & 0.0273 & 0.0525 & 0.0286 & 0.0831 & 0.1551 & $8.2744 \mathrm{e}-004$ \\
\hline $2(\mathrm{EX)}$ & 0.0227 & 0.0461 & 0.0291 & 0.0884 & 0.1773 & $5.9914 \mathrm{e}-004$ \\
\hline $3(\mathbf{P T})$ & 0.0319 & 0.0583 & 0.0280 & 0.0807 & 0.1389 & 0.0011 \\
\hline $\mathbf{4}$ (TR) & 0.0228 & 0.0465 & 0.0338 & 0.1076 & 0.2070 & $6.3135 \mathrm{e}-004$ \\
\hline $5(\mathbf{I T})$ & 0.0340 & 0.0625 & 0.0386 & 0.1114 & 0.1858 & 0.0013 \\
\hline
\end{tabular}

Given these observations, the following generalizations are advanced for the case in which the monetary authority has access to full contemporaneous information:

First, in our model the Taylor rule always performs better than if the monetary policy rule is augmented by some form of the exchange rate, irrespective of which particular version of the model used. ${ }^{63}$ What is important to note here is that this conclusion arises despite the more general nature of the loss function (derived in Chapter 3) used in this analysis that is not standard for the literature. That is, most often the welfare loss is evaluated in terms of a loss function that incorporates only variations of the inflation rate and the output gap (see Rotemberg and Woodford, 1999, Adolfson, 2005) and here the Taylor Rule is frequently found to perform best. However, our

\footnotetext{
${ }^{63}$ In the literature it is an open issue how exchange rate should be taken into account in formulating monetary policy (see Ball, 1999, Svensson, 2000, and Taylor, 2001).
} 
measure incorporates all the arguments in the utility function and hence incorporates dimensions not always considered. Even here we have found the Taylor Rule to perform best. Hence the speculation in the literature that the Taylor Rule may work well even for small open economies may be more robust than was previously appreciated. ${ }^{64}$

Second, when the monetary authority has access to sufficient contemporaneous information to determine the flexible price values of the model's endogenous variables, the adoption of interest rate smoothing only detracts from the power of monetary policy. That is, while interest-rate smoothing does in some situations decrease the variation in interest rates, they also increase the variance of all other variables, i.e., the inflation rate, the output gap, the real exchange rate, and the law of one price gap. Given access to the appropriate contemporaneous information, the result of interest rate smoothing is to move these variables away from their appropriate flexible price values and so decrease the overall level of welfare experienced by households in the community. Hence if the central bank had sufficient information, it would be neither necessary nor desirable for the central bank to respond to lagged interest rates.

Third, when the monetary authority maintains a long run steady state rate of inflation of zero, whether the monetary authority can improve performance by targeting the inflation gap rather than the inflation rate is ambiguous. For our model, the variance in inflation and other variables becomes larger when the policy maker reacts to the inflation gap using output prices as the measure of inflation, but becomes smaller when the policy maker uses a consumer price measure and reacts to the inflation gap.

\footnotetext{
${ }^{64}$ If we narrow our welfare criterion to this smaller set of variables and evaluate our alternative policy rules, we can still find that the Taylor rule works better in most situations.
} 
Fourth, while policy response to CPI measure of inflation lowers the variation in real exchange rates and the law of one price gap, it also increases the variance of inflation, the output gap, and the interest rate. In our model this results in a larger welfare loss compared to the case in which policy maker responds to a domestic output price measure. While this finding is of important policy significance, this ranking is dependent on the weights assigned to the separate terms in the policy function, weights that have been assigned on the basis of values often chosen in the literature (rather than solved endogenously). Hence it will be of interest to see if this ranking is maintained when the response coefficients are solved endogenously in Chapter 5.

Finally, what will become more apparent as we move through the following cases is that the Wicksellian interest rate, $\hat{r}_{H, t}{ }^{f}$, is the crucial piece of information needed to make monetary policy more effective. ${ }^{65}$ Even though the use of the Wicksellian interest rate cannot completely stabilize inflation and the output gap, an easy substitute for the Wicksellian interest rate is not readily available. In the cases below, we show that once $\hat{r}_{H, t}{ }^{f}$ becomes unavailable, the substitution of another interest rate target for $\hat{r}_{H, t}{ }^{f}$ dramatically increases the variance of the model's endogenous variables rise and results in large household welfare falls losses.

\section{Case 2: Comparative performance when monetary policy can use only contemporaneous departures from steady state values}

Case 1 assumes that an extraordinary amount of timely information is available to the central bank. In order to estimate $\hat{Y}_{i}^{f}$, for example, the central bank needs accurate

\footnotetext{
${ }^{65}$ In what follows we will see that the level of output arising from the perfect flexible prices, $\hat{Y}_{t}^{f}$, is also a piece of important information needed make the monetary policy more effective.
} 
information on the realization of three out of the four shocks that affect the model ${ }^{66}$ The requirements for extracting this information perfectly from the contemporaneous information available are too stringent to expect to be satisfied. One possibility that we will explore later is that the monetary authority can observe or infer only part of each shock implying that $\hat{Y}_{t}^{f}$ and $\hat{q}_{t}^{f}$ can be determined only imperfectly. Suppose however, that the best that the monetary authority can do is determine the steady state values of all variables in the system. In this case, equation (4.1), the general form of the monetary policy rule using domestic output price inflation becomes

$$
\hat{R}_{t}=\left(1-\phi_{r}\right)\left\lfloor\hat{\Pi}_{H, t}+\phi_{\pi} \hat{\Pi}_{H, t}+\phi_{x} \hat{Y}_{t}+\phi_{q} \hat{q}_{t}+\phi_{\Psi} \hat{\Psi}_{t}\right\rfloor+\phi_{r} \hat{R}_{t-1}
$$

and the corresponding equation using consumer prices can be rewritten as

$$
\left.\hat{R}_{t}=\left(1-\phi_{r}\right) \mid \hat{\Pi}_{t}+\phi_{\pi} \hat{\Pi}_{t}+\phi_{x} \hat{Y}_{t}+\phi_{q} \hat{q}_{t}+\phi_{\Psi} \hat{\Psi}_{t}\right\rfloor+\phi_{r} \hat{R}_{t-1}
$$

where the hats over the variables represent the log-deviation of the variable from its steady state value rather than the gap between flexible and sticky price outcomes. ${ }^{67}$

The comparative performance of the policy rules for Case 2 is summarized in Tables 4.11 through 4.14 . In general terms, the dynamic impulse responses of the model's key endogenous variables to the model's shocks and the relative rankings under the alternative policy rules in Case 2 are considerably different from those found in Case 1. The following are some of the most important differences that emerged in Case 2.

We begin by ignoring temporarily the interest rate smoothing results to focus on the set of standard monetary policy rules in Table 4.11. Observing statistics listed in

\footnotetext{
${ }^{66}$ The risk premium shock does not affect the output arising under the perfectly flexible prices and complete asset markets.

${ }^{67}$ The simulation results associated with alternative rules in Case 2 when CPI inflation is used are roughly similar to those obtained in Case 1, they are placed in Appendix 4A.1. Similarly we put the results when CPI prices are used in Cases 3 and 4 in Appendix 4A.2 and 4A.3, respectively.
} 
Table 4.11, we see that the first three rules that involve the openness of the economy and some measure of the exchange rate generally work very well in comparison with the Taylor and inflation targeting rules. This is opposite to what we discovered in Case 1. As might be expected, the need to use less relevant information means that the standard deviations of all variables under all alternative policy rules in Case 2 are larger than those in Case 1. This in turn implies that the welfare losses are all larger. For example, compared the smallest welfare loss in Table 4.11 to the one in Table 4.3 , the former is 38.4 times larger than the later one. In this case, then, the ability to incorporate more instruments into the policy rule allows the monetary authority to improve on overall performance.

Second, when we compare the three quasi-exchange rate rules, the standard deviations of all key endogenous variables under Rule 3 are all smaller and hence household welfare higher under Rule 3 than under the two other rules. This is completely different from our finding in Case 1. There the standard deviations in Rule 2 were always smaller than those under Rules 1 and 3, and the level of welfare correspondingly higher.

Third, the standard deviations for the real exchange rate and law of one price gap were always larger under the Taylor rule in Case 1 than those corresponding to the first three rules, while the standard deviations of the inflation, output gap and interest rate under the Taylor rule in Case 1 were all smaller. This resulted in the Taylor rule having the smaller welfare loss. In Case 2, however, even though the Taylor rule results in a smaller standard deviation for the output gap, the Taylor rule induces larger variations for all other variables relative to the first three exchange rate oriented rules. This implies that even if we used the conventional loss function (which incorporates only the inflation rate 
and the output gap) to evaluate the alternative policy rules, the Taylor rule cannot be recommended. Moreover, Case 2 shows that targeting only the inflation rate in Rule 5 now dominates the Taylor rule. Inflation targeting yields only a slightly larger standard deviation for inflation and the output gap in comparison to Rules 1 and 3 and hence results in only a slightly lower level of welfare.

Table 4.11

Case 2: Targeting inflation measured in domestic output prices

\begin{tabular}{l|l|l|l|l|l|l}
\hline Policy Rule & \multicolumn{5}{|c|}{ Standard Deviation } & Welfare \\
\cline { 2 - 6 } & $\hat{\Pi}_{H, t}$ & $x_{t}$ & $R_{H g, t}$ & $q_{g, t}$ & $\hat{\Psi}_{t}$ & loss \\
\hline 1 (C) & 0.0884 & 0.0767 & 0.0584 & 0.1567 & 0.2628 & 0.0081 \\
\hline 2 (EX) & 0.1127 & 0.0805 & 0.0815 & 0.2021 & 0.3583 & 0.0133 \\
\hline 3 (PT) & 0.0729 & 0.0732 & 0.0450 & 0.1278 & 0.2047 & 0.0055 \\
\hline 4 (TR) & 0.1435 & 0.0672 & 0.1206 & 0.2560 & 0.4902 & 0.0219 \\
\hline 5 (IT) & 0.1023 & 0.0423 & 0.0874 & 0.2188 & 0.4569 & 0.0112 \\
\hline
\end{tabular}

When we now add interest rate smoothing to the policy mix (compare Table 4.12 with Table 4.11), the one overwhelming conclusion is that the addition of interest rate smoothing to all the other monetary rules improves performance. Compared to the outcomes under non-interest rate smoothing in Table 4.11, welfare losses can be reduced from approximately $11 \%$ (in Rule 3 ) to $36 \%$ (under Rule 5). However, while interest rate smoothing improves all levels of performance, it does not affect relative performance. ${ }^{68}$ Among the interest-rate smoothing rules, Rule 3 once again yields the best performance result.

\footnotetext{
${ }^{68} \mathrm{McC}$ Callum and Nelson (1999) derive a similar conclusion. They find that an interest rate smoothing term in the Taylor rule helps to reduce the variability of inflation and output gap for given values of the policy response parameters, while also reducing $R_{t}$ variability. This finding holds for both variants of their model (with Calvo-Rotemberg pricing and the P-bar price adjustment relation).
} 
Table 4.12

Case 2: Inflation measured in domestic output prices and interest rate smoothing

\begin{tabular}{l|l|l|l|l|l|l}
\hline \multirow{2}{*}{ Policy Rule } & \multicolumn{5}{|c|}{ Standard Deviation } & Welfare \\
\cline { 2 - 7 } & $\hat{\Pi}_{H, t}$ & $x_{t}$ & $R_{H g, t}$ & $q_{g, t}$ & $\hat{\Psi}_{t}$ & loss \\
\hline $\mathbf{1}(\mathbf{C})$ & 0.0829 & 0.0947 & 0.0396 & 0.1551 & 0.2385 & 0.0070 \\
\hline $\mathbf{2}(\mathbf{E X )}$ & 0.1031 & 0.1050 & 0.0520 & 0.1895 & 0.3072 & 0.0109 \\
\hline $\mathbf{3}(\mathbf{P T})$ & 0.0693 & 0.0871 & 0.0328 & 0.1329 & 0.1975 & 0.0049 \\
\hline $\mathbf{4}(\mathbf{T R})$ & 0.1199 & 0.1100 & 0.0679 & 0.2255 & 0.3897 & 0.0148 \\
\hline $\mathbf{5}(\mathbf{I T})$ & 0.0841 & 0.1047 & 0.0210 & 0.2329 & 0.4456 & 0.0072 \\
\hline
\end{tabular}

\subsection{Comparative Performance when Data Availability Allows only Lagged}

\section{Response}

The information that the monetary authority needs to make its policy decision can be classified into two categories: one category is observations on where the economic system is now, such as the current level of nominal and real GDP; the other is the unobservable variables that need to be inferred or estimated, such as the Wicksellian interest rate and flexible price output. In the previous sections we allowed the monetary authority to access all current-period information and for policy to respond immediately to the setting of short-term interest rates. The problem posed in Case 2 was that there was the flexible price variables in the targeted gaps could not be calculated from the information available. In this section we follow Taylor (1999) and many others, to argue that policymakers may not even have accurate information on the current state of the system when they make their interest rate decision. That is, in general it is unreasonable to expect that policymakers can access all needed information with no time lag. The aggregate information needed takes time to collect and process, and the time and effort 
needed to collect and process unobservable conceptual information would take even longer, perhaps even being impossible to collect. Moreover, even when the relevant information is collected, policymakers still need time to decide on an appropriate response to that information. Finally, as McCallum and Nelson (1999) have argued, there is considerable uncertainty regarding the realized value of real GDP even at the end of the quarter in actual economies. In this sense, the policy rule specified by Taylor (1993) is not fully operational. ${ }^{69}$ In this section we assume that the monetary authority must respond in setting its interest rate today only to lagged information (which we assume is accurate). The specific question we are interested in is, does having to use lagged information affect the ranking of the monetary policy rules already considered?

\section{Case 3: Policy response under lagged information}

Case 3 differs from Case 1 in that the monetary authority can now access all relevant information accurately but only after a one period lag. In Cases 1 and 2, monetary policy could be based on observed current variables and policy was implemented contemporaneously. In Case 3 we assume that because of lagged information and/or transmission lags, the monetary authority must use a forward-looking perspective. And, as Keynes (1923) wryly observed, "If we wait until a price movement is actually afoot before applying remedial measures, we may be too late!" In the first instance, the monetary authority may use what is implicitly dated older information as if it were current. However, it is perhaps more likely that the monetary authority will use

\footnotetext{
${ }^{69}$ Taylor $(1999, \mathrm{p} 12)$ argues that a lagged version of his policy rule, e.g., $i_{t}=\phi_{\pi} \pi_{t-1}+\phi_{x} y_{t-1}+\phi_{r} i_{t-1}$, where $i_{t}$ denotes the short-term interest rate, is any more realistic than the contemporaneous version, $i_{t}=\phi_{\pi} \pi_{t}+\phi_{x} y_{t}+\phi_{r} i_{t-1}$, because policymakers will always have some current period information available when they make interest rate decisions.
} 
that information to form a forecast of what inflation is likely to be when monetary policy comes into effect. In our current model this means the authority makes a forecast of what the unobserved current inflation rate is now when policy is implemented (without a lag). This gives us one additional monetary policy rule. Hence we call Rule 6, a strict inflation forecast targeting rule (SIF), where in this case the output gap does not show up in the rule. Batini and Haldane (1999) argue that this rule is output encompassing since the targeting horizon and feedback parameter can, in principle, secure a degree of output smoothing. On the other hand, Clarida et al. $(1998,2000)$ take explicit account of the output gap objective and find this forwarding looking rule fits recent monetary policy history quite well. Hence following Clarida et al. $(1998,2000)$ we obtain Rule 7 by adding the term the output gap into Rule 6, we call it an inflation-forecast Taylor rule (IFTR).

For Case 3, then, the general form of monetary policy rules becomes,

$$
\hat{R}_{t}=\left(1-\phi_{r}\right)\left[\begin{array}{l}
\hat{r}_{H, t-1}^{f}+\hat{\Pi}_{H, t-1}+\phi_{\pi}\left(\hat{\Pi}_{H, t-1}-\hat{\Pi}_{H, t-1}^{f}\right) \\
+\phi_{f}\left(\hat{\Pi}_{H, t+j, t}-\hat{\Pi}_{H, t-1}^{f}\right)+\phi_{x} x_{t-1}+\phi_{q} q_{g t-1}+\phi_{\Psi} \hat{\Psi}_{t-1}
\end{array}\right]+\phi_{r} \hat{R}_{t-2},
$$

where the values of response coefficients once again are given in Table 4.2.

Tables 4.13 through 4.16 report the standard deviations and welfare losses associated with the corresponding policy rules conducted by the monetary authority under the information constraints of Case 3. Specifically, Table 4.13 assumes that the monetary authority makes its current response to lagged information using the domestic output-price measure of inflation and without interest rate smoothing. The solutions in Table 4.13 the following special features. First, comparing the first three exchange rate related rules with the remaining four non-exchange rate related rules, we see that the 
exchange rate rules yield larger welfare losses. Second, among these four non-exchange rate rules, the Taylor rule generates the smallest variance for inflation and the output gap and hence the smallest overall level of welfare loss despite having a higher standard deviation for the interest rate. Third, a comparison of Rule 7 to Rule 4 shows that inflation-forecast-based Taylor rule does not improve the outcome relative to its simple counterpart (Rule 4, the Taylor rule) and the same conclusion can be drawn for inflation targeting by comparing the outcome of Rule 6 to that of Rule 5 .

What is perhaps surprising is that the findings reproduced in these tables are still not new, again repeating the general pattern found in Case 1 (and to some extent, Case 2). The obvious exception is that variances of the variables are now all larger and the levels of welfare realized somewhat lower. For example, the smallest welfare loss yielded by Rule 4 in Table 4.13 is $2.3807 \mathrm{e}-004$, while in Table 4.3 is $1.3969 \mathrm{e}-004$. Here the lack of information can be blamed with poorer information resulting in a $70 \%$ larger welfare loss. In both Cases 2 and 3 there are information shortfalls. However, when the performance outcomes of Table 4.13 are compared to their counterpart in Table 4.11, the overall policy performance in Case 3 turns out to be much better than that in Case 2 . This suggests that there is less relevant information lost and hence better performance outcomes achieved when older 'actual' information is used than when we simply substitute the steady state values for the flexible price alternative in the policy rule. 
Table 4.13

Case 3: Policy uses domestic output price inflation

\begin{tabular}{l|l|l|l|l|l|l}
\hline \multirow{2}{*}{$\begin{array}{l}\text { Policy } \\
\text { Rule }\end{array}$} & \multicolumn{5}{|c|}{ Standard Deviation } & \multirow{2}{*}{ Welfare loss } \\
\cline { 2 - 6 } & $\hat{\Pi}_{H, t}$ & $x_{t}$ & $R_{H g, t}$ & $q_{g, t}$ & $\hat{\Psi}_{t}$ & \\
\hline $\mathbf{l}$ (C) & 0.0187 & 0.0385 & 0.0476 & 0.0844 & 0.1757 & $5.5732 \mathrm{e}-004$ \\
\hline $\mathbf{2}$ (EX) & 0.0145 & 0.0340 & 0.0481 & 0.0917 & 0.1987 & $4.2217 \mathrm{e}-004$ \\
\hline 3 (PT) & 0.0237 & 0.0433 & 0.0475 & 0.0797 & 0.1582 & $7.6888 \mathrm{e}-004$ \\
\hline 4 (TR) & 0.0096 & 0.0332 & 0.0393 & 0.1158 & 0.2410 & $2.3807 \mathrm{e}-004$ \\
\hline 5 (IT) & 0.0139 & 0.0390 & 0.0348 & 0.1206 & 0.2357 & $3.1060 \mathrm{e}-004$ \\
\hline $\mathbf{6 ( S I F )}$ & 0.0190 & 0.0429 & 0.0370 & 0.1257 & 0.2389 & $4.9344 \mathrm{e}-004$ \\
\hline $\mathbf{7}$ (IFTR) & 0.0120 & 0.0337 & 0.0392 & 0.1171 & 0.2429 & $2.8941 \mathrm{e}-004$ \\
\hline
\end{tabular}

Table 4.14 shows the results when interest-rate smoothing is again added to each of the monetary policy rules used in Table 4.13. In this case, however, the first three exchange rate rules now generate the smaller variable variances and hence realize higher welfare levels (in direct contrast to Table 4.13). Second, when we compare Table 4.14 to 4.13 , we see that interest rate smoothing does not improve the policy performance at all and this result extends to the other interest rate smoothing case shown in Tables 4.16. This finding, robust across all policy rules in Case 3 is a bit surprisingly, since in Case 2 interest-rate smoothing did help improve policy performance. One possible interpretation of this result, similar to that given for the general superiority of Case 3 outcomes over Case 2 , is that even though both cases have an information shortfall (relative to complete information), the information in the variables used in Case 3 is more informative than that used in Case 2 for combating the gaps between actual and flexible price outcomes. In this reading of the results, the separate information contained in using the lagged values of the Wicksellian natural rate, the flexible level of output, $\hat{Y}_{t}^{f}$, and the flexible real 
exchange rate, $\hat{q}_{t}{ }^{f}$, are more helpful for closing the various gaps than the information that can be gained by using an aggregated version of this information in the lagged interest rate. $^{70}$

Table 4.14

Case 3: The domestic inflation with interest-rate smoothing

\begin{tabular}{l|l|l|l|l|l|l}
\hline \multirow{2}{*}{ Policy Rule } & \multicolumn{5}{|c|}{ Standard Deviation } & \multirow{2}{*}{ Welfare loss } \\
\cline { 2 - 6 } & $\hat{\Pi}_{H, t}$ & $x_{t}$ & $R_{H g, t}$ & $q_{g, t}$ & $\hat{\Psi}_{t}$ & \\
\hline $\mathbf{1}$ (C) & 0.0240 & 0.0499 & 0.0435 & 0.1121 & 0.2123 & $7.5497 \mathrm{e}-004$ \\
\hline 2 (EX) & 0.0227 & 0.0479 & 0.0441 & 0.1216 & 0.2372 & $6.9963 \mathrm{e}-004$ \\
\hline 3 (PT) & 0.0268 & 0.0528 & 0.0430 & 0.1059 & 0.1938 & $8.9334 \mathrm{e}-004$ \\
\hline 4 (TR) & 0.0298 & 0.0505 & 0.0468 & 0.1431 & 0.2773 & 0.0011 \\
\hline 5 (IT) & 0.0338 & 0.0584 & 0.0495 & 0.1464 & 0.2717 & 0.0014 \\
\hline 6 (SIF) & 0.0385 & 0.0628 & 0.0543 & 0.1528 & 0.2802 & 0.0018 \\
\hline 7 (IFTR) & 0.0337 & 0.0521 & 0.0504 & 0.1480 & 0.2863 & 0.0014 \\
\hline
\end{tabular}

Tables 4.15 and 4.16 summarize the simulation results when the policy maker responds to the inflation gap rather than the inflation rate itself. Comparing these results with those in Tables 4.13 and 4.14, it can be seen that targeting the lagged inflation gap rather than the lagged rate of inflation does not reduce the variances of the key variables nor increase welfare levels.

\footnotetext{
${ }^{70}$ Our results also show that use by the monetary authority of the one-period lagged interest rate instead of the two-period lagged interest rate as used here does not affect this conclusion.
} 
Table 4.15

Case 3: Targeting the domestic output inflation gap

\begin{tabular}{l|l|l|l|l|l|l}
\hline \multirow{2}{*}{ Policy Rule } & \multicolumn{5}{|c|}{ Standard Deviation } & \multirow{2}{*}{ Welfare loss } \\
\cline { 2 - 6 } & $\hat{\Pi}_{H, t}$ & $x_{t}$ & $R_{H g, t}$ & $q_{g, t}$ & $\hat{\Psi}_{t}$ & \\
\hline $\mathbf{1}(\mathbf{C})$ & 0.0227 & 0.0589 & 0.0754 & 0.0805 & 0.1641 & 0.0010 \\
\hline $\mathbf{2}$ (EX) & 0.0214 & 0.0541 & 0.0786 & 0.0875 & 0.1913 & 0.0010 \\
\hline $\mathbf{3}$ (PT) & 0.0267 & 0.0638 & 0.0739 & 0.0773 & 0.1444 & 0.0012 \\
\hline 4 (TR) & 0.0237 & 0.0515 & 0.0752 & 0.1099 & 0.2374 & 0.0011 \\
\hline $\mathbf{5}$ (IT) & 0.0233 & 0.0614 & 0.0688 & 0.1096 & 0.2182 & $9.7658 \mathrm{e}-004$ \\
\hline $\mathbf{6}$ (SIF) & 0.0209 & 0.0423 & 0.0362 & 0.1404 & 0.2821 & $5.6631 \mathrm{e}-004$ \\
\hline $\mathbf{7}$ (IFTR) & 0.0225 & 0.0338 & 0.0422 & 0.1306 & 0.2795 & $6.7530 \mathrm{e}-004$ \\
\hline
\end{tabular}

Table 4.16

Case 3: Domestic output inflation gap with interest rate smoothing

\begin{tabular}{l|l|l|l|l|l|l}
\hline \multirow{2}{*}{ Policy Rule } & \multicolumn{5}{|c|}{ Standard Deviation } & \multirow{2}{*}{ Welfare loss } \\
\cline { 2 - 6 } & $\hat{\Pi}_{H, t}$ & $x_{t}$ & $R_{H g, t}$ & $q_{g, t}$ & $\hat{\Psi}_{t}$ & \\
\hline $\mathbf{1}$ (C) & 0.0245 & 0.0540 & 0.0495 & 0.1057 & 0.2002 & $8.2907 \mathrm{e}-004$ \\
\hline $\mathbf{2}$ (EX) & 0.0230 & 0.0505 & 0.0508 & 0.1146 & 0.2262 & $7.6960 \mathrm{e}-004$ \\
\hline $\mathbf{3}$ (PT) & 0.0276 & 0.0579 & 0.0488 & 0.1003 & 0.1813 & $9.8485 \mathrm{e}-004$ \\
\hline 4 (TR) & 0.0287 & 0.0506 & 0.0512 & 0.1352 & 0.2665 & 0.0011 \\
\hline 5 (IT) & 0.0318 & 0.0590 & 0.0509 & 0.1362 & 0.2545 & 0.0013 \\
\hline 6 (SIF) & 0.0378 & 0.0564 & 0.0494 & 0.1662 & 0.3266 & 0.0017 \\
\hline $\mathbf{7}$ (IFTR) & 0.0415 & 0.0674 & 0.0527 & 0.1773 & 0.3363 & 0.0020 \\
\hline
\end{tabular}

Case 4: Monetary policy when the monetary authority can use only lagged departures from steady state values

In case 3 we assumed that the monetary authority can access both observable variables, such as $\hat{\Pi}_{H, t-1}$, and unobservable variables, such as the Wicksellian natural 
rate, with a one period lag. However, while it may be reasonable to assume that the policy maker can obtain all observable information with some lag, it is unlikely that the policy maker may have enough information to calculate the unobservable variables even if it had sufficient time. If this is true, then the policy maker cannot calculate the flexible price values and must respond in setting its interest rate only to lagged observable information. This gives us Case 4 , which is very close to case 2 but uses $\hat{\Pi}_{H, t-1}, \hat{Y}_{t-1}$, $\hat{q}_{t-1}, \hat{\Psi}_{t-1}$, and $\hat{R}_{t-1}$ to replace $\hat{\Pi}_{H, t}, \hat{Y}_{t}, \hat{q}_{t}, \hat{\Psi}_{t}$, and $\hat{R}_{t}$ in the general policy form (4.5). Hence the policy response function in (4.5) will be modified to,

$$
\hat{R}_{t}=\left(1-\phi_{r}\right)\left[\hat{\Pi}_{H, t-1}+\phi_{\pi} \hat{\Pi}_{H, t-1}+\phi_{f} \hat{\Pi}_{H, t+j \mid t}+\phi_{x} \hat{Y}_{t-1}+\phi_{q} \hat{q}_{t-1}+\phi_{\Psi} \hat{\Psi}_{t-1}\right]+\phi_{r} \hat{R}_{t-2}
$$

The simulation results associated with alternative policy rules based on equation (4.6) are summarized in Tables 4.17 and 4.18. Examining them we see that the general pattern of the policy performance corresponding to each rule replicates the findings in Case 2. For instance, Rule 3 where the policy maker responds to the law of one price gap again achieves the best welfare performance in all two situations (Tables 4.17 and 4.18). Similarly, the performance of monetary policy rule improves under interest-rate smoothing rules as compared to the same cases without interest rate smoothing. One unexpected result is that the size of the welfare losses incurred in Case 4 is almost same as the losses incurred in Case 2-suggesting that the important information loss is in the inability to calculate the flexible price values rather than the loss of current realized values. Overall, the smallest welfare loss arising in Case 4 is 0.005 , generated by Rule 3 when the monetary authority uses the domestic output-price measure of inflation together with interest rate smoothing. In Case 2, the smallest welfare loss is 0.0049 . 
Table 4.17

Case 4: Inflation measured in domestic output prices

\begin{tabular}{l|l|l|l|l|l|l}
\hline \multirow{2}{*}{$\begin{array}{l}\text { Policy } \\
\text { Rule }\end{array}$} & \multicolumn{5}{|c|}{ Standard Deviation } & \multirow{2}{*}{ Welfare loss } \\
\cline { 2 - 6 } & $\hat{\Pi}_{H, t}$ & $x_{t}$ & $R_{H g, t}$ & $q_{g, t}$ & $\hat{\Psi}_{t}$ & \\
\hline $\mathbf{1}$ (C) & 0.0844 & 0.0858 & 0.0699 & 0.1547 & 0.2505 & 0.0076 \\
\hline $\mathbf{2}$ (EX) & 0.1064 & 0.0925 & 0.0880 & 0.1948 & 0.3343 & 0.0120 \\
\hline $\mathbf{3 ( \mathbf { P T } )}$ & 0.0701 & 0.0806 & 0.0601 & 0.1290 & 0.1994 & 0.0053 \\
\hline $\mathbf{4}$ (TR) & 0.1284 & 0.0862 & 0.1109 & 0.2347 & 0.4343 & 0.0176 \\
\hline $\mathbf{5}$ (IT) & 0.0843 & 0.0695 & 0.0745 & 0.1995 & 0.4141 & 0.0076 \\
\hline $\mathbf{6}$ (SIF) & 0.1184 & 0.0770 & 0.0915 & 0.2482 & 0.5164 & 0.0148 \\
\hline $\mathbf{7}$ (IF) & 0.1757 & 0.0995 & 0.1420 & 0.3119 & 0.5944 & 0.0310 \\
\hline
\end{tabular}

Table 4.18

Case 4: Lagged domestic output inflation with interest rate smoothing

\begin{tabular}{l|l|l|l|l|l|l}
\hline \multirow{2}{*}{ Policy Rule } & \multicolumn{5}{|c|}{ Standard Deviation } & \multirow{2}{*}{ Welfare loss } \\
\cline { 2 - 6 } & $\hat{\Pi}_{H, t}$ & $x_{t}$ & $R_{H g, t}$ & $q_{g, t}$ & $\hat{\Psi}_{t}$ & \\
\hline $\mathbf{1}$ (C) & 0.0823 & 0.1056 & 0.0505 & 0.1611 & 0.2381 & 0.0070 \\
\hline $\mathbf{2}$ (EX) & 0.1010 & 0.1188 & 0.0582 & 0.1914 & 0.2944 & 0.0105 \\
\hline $\mathbf{3}$ (PT) & 0.0694 & 0.0964 & 0.0464 & 0.1419 & 0.2057 & 0.0050 \\
\hline $\mathbf{4}$ (TR) & 0.1149 & 0.1303 & 0.0654 & 0.2248 & 0.3641 & 0.0136 \\
\hline $\mathbf{5}$ (IT) & 0.0877 & 0.1343 & 0.0356 & 0.2601 & 0.4703 & 0.0079 \\
\hline $\mathbf{6}$ (SIF) & 0.1220 & 0.1770 & 0.0346 & 0.3323 & 0.5913 & 0.0151 \\
\hline $\mathbf{7}$ (IF) & 0.1454 & 0.1623 & 0.0712 & 0.2811 & 0.4613 & 0.0217 \\
\hline
\end{tabular}

\subsection{Concluding Remarks}

In this chapter we compared the simulated outcomes of a set of monetary policy rules for the special case where the reaction coefficients used in the policy rule were the ones that have typically been used in the literature. Beginning from the case of full 
contemporaneous information, we took information away from the monetary authority and looked at how the performance of our alternative monetary policy rules performed on this basis. In terms of these experiments, the following hypotheses seem to be warranted.

First, the information available to the monetary authority in setting its policy rule really does matter. On the one hand, the amount of information not only affects the level of welfare achieved by the policy, but affects which policy rule will likely perform best. As one would expect, the more information the monetary authority can access the larger will the welfare gain be to monetary policy. For example, the monetary authority has access to the most relevant information in Case 1 and the least information in Case 4 . Correspondingly, we find the smallest welfare loss in Case 1, that is, $1.3969 \mathrm{e}-004$, and the smallest loss in Case 4, that is, 0.0050 . In addition, the amount of information available influences the performance of each rule. Consider, for example, the situation in which the policy maker uses domestic output prices to measure inflation and doesn't adopt interest rate smoothing. For this situation, we see either when all relevant information is readily available (Case 1) or available with a one period lag (Case 3 ), then the Taylor rule (Rule 4) is the monetary policy rule that generates the smallest expected welfare loss. On the other hand, when the information available is insufficient to allow the flexible price values to be calculated either in the present (Case 2) or even with a one period lag (Case 4) it is the monetary policy rule using the incomplete pass gap (Rule 3) that works best.

Second, for the literature as a whole whether the exchange rate needs to be in the monetary policy rule or not is still an open question. Unfortunately, our analysis does not provide a clear cut answer. Whether the use of the exchange rate in the monetary policy 
rule improves performance outcomes depends upon several factors, in particular, the information set available to the policy maker and whether interest rate smoothing is to be included. In the cases we have considered that do not use interest rate smoothing, the simulation results indicate that only in Cases 2 and 4 does one of the exchange rate augmenting rules produce minimum variances in the model's key efficiency gaps and hence provide for the best enhancement of social welfare. In Cases 1 and 3 , the Taylor rule (excluding open economy considerations) performs best. However when the monetary authority considers interest rate smoothing as part of its policy rule, in each of the four cases, at least one of the exchange rate augmenting rules (Rules 2 and 3) will perform best.

Third, the effect of interest-rate smoothing on policy performance is ambiguous. The simulations from Cases 1 and 3 show that the addition of an interest rate smoothing term reduces monetary policy performance by introducing additional volatility into the key variables and dramatically reducing potential social welfare. Particularly for the inflation rate and output gap under the Taylor rule, their respective standard deviations rise from 0.0096 and 0.0242 in Case 1 (see Table 4.3) to 0.0202 and 0.0399 (see Table 4.4) after the interest-rate smoothing is incorporated. On the other hand, the interest rate smoothing does unambiguously improve the policy performance in Cases 2 and 4 . This persistent pattern suggests that it is only when stationary state values are used to target monetary policy that the lagged interest rate allows some part of the persistence characteristics of the models shocks to be incorporated into the performance rule and so improve welfare. Along the same lines, it may be that because the lagged interest rate incorporates these concerns in a somewhat cruder (more aggregated) way that the use of 
either the contemporary or the lagged values of the flexible price variables directly can better incorporate the disaggregated aspects of persistence and so improve overall performance. In this sense, the adoption of interest rate smoothing would then require policy to use a less informative statistic that would, in turn, reduce rather than enhance policy performance. This would be consistent with the general idea that the role of interest rate smoothing in monetary policy increases with the amount of current relevant information lost to the policy maker.

Fourth, while domestic output inflation is identical to CPI inflation in a closed economy, the two measures differ for a small open economy. The question then arises whether the policy maker should be directed to respond to domestic output-price or CPI inflation. Our simulation results imply that when the policy maker uses reaction coefficient close to those typically used in the literature that the monetary authority should respond to the variation in domestic output price inflation rather than CPI inflation.

Fifth, in the current literature the inflation rate and the inflation rate gap are conflated, implying that the inflation rate that arises under perfectly flexible prices is always zero. Our analysis suggests that this assumption is incorrect and our simulations imply that this approximation is not always appropriate. In particular, our simulations find that when the monetary authority uses the domestic output price measure of inflation and targets monetary policy on the inflation rate gap rather than the inflation rate, the performance of monetary policy will fall. On the other hand, when the monetary authority uses the CPI inflation measure, then targeting the inflation rate gap does improve somewhat monetary policy relative to inflation rate targeting. 
Finally, in the case where the monetary authority has access to accurate information only with a one period lag, we find that neither of our two inflation forecast rules enhances social welfare relative to the monetary authority's use of the lagged policy variables themselves. This is an unexpected finding and one that we will be interested in pursuing as we move to more a more general setting for our policy rules.

Before concluding this chapter we should reinforce the starting observation that all these conclusions on the relative strength of different monetary policy rules are highly conditional. This is because the policy response coefficients used in the simulations above, while typical of those used in the literature, are not necessarily optimal for each specific policy rule, the model developed and/or the information structure assumed. Hence while the coefficients used are not completely arbitrary, the conclusions with respect to which policy rule works best may well change when we move to adopt "optimal" policy response coefficients in the chapter that follows. However, given the widespread use in practice of reaction coefficients close to these assumed values, this analysis does suggest caution in simply adopting simple monetary rules such as strict inflation targeting and/or the Taylor rule. Nor does it seem wise to simply assume that all small open economies should incorporate some form of an exchange rate term. Because even these conclusions are of immediate policy interest, it will be interesting to see if even these suggestions survive into the setting of "optimal" reaction coefficients. 


\section{Appendix to Chapter 4}

\section{A4.1 Simple policy rules in Case 2 where the monetary authority cannot calculate the fully flexible price values need for the appropriate gaps}

Tables A4.1 and A4.2 summarize the simulation results for when the monetary authority targets inflation in terms of the consumer price measure of inflation. When we look across these tables, we see that Rule 3 again produces the smallest variance for the key endogenous variables and therefore results in the smallest welfare loss across the set of money rules. In addition, the use of interest rate smoothing produces better policy performance for each monetary policy rule. Hence the results for consumer prices are consistent with the results obtained for the domestic output price measure of inflation (Tables 4.11 and 4.12) and hence reinforce the generality of these findings. Finally, if we compare the welfare results for the use of consumer prices in Tables A4.1 and A4.2 with the results when producer prices are used in Tables 4.11 and 4.12, we find that both the standard deviations of the key variables and the welfare losses are somewhat larger for consumer prices. This is the same result as the one we found for the case of full contemporaneous information in Case 1 . Hence in the case when the monetary authority cannot calculate or observe the flexible price levels consistent with efficiency, the recurring dominance of producer prices reinforces the earlier case made for the use of domestic output prices (over consumer prices) in the monetary rule. 
Table A4.1

Case 2: consumer prices (CPI) used to measure inflation rates

\begin{tabular}{c|c|c|c|c|c|c}
\hline \multirow{2}{*}{ Policy Rule } & \multicolumn{5}{|c|}{ Standard Deviation } & Welfare \\
\cline { 2 - 6 } & $\hat{\Pi}_{H, t}$ & $x_{i}$ & $R_{g, t}$ & $q_{g, t}$ & $\hat{\Psi}_{t}$ & loss \\
\hline 1 (C) & 0.0918 & 0.0962 & 0.0579 & 0.1588 & 0.2459 & 0.0088 \\
\hline 2 (EX) & 0.1157 & 0.1044 & 0.0792 & 0.2055 & 0.3425 & 0.0140 \\
\hline 3 (PT) & 0.0765 & 0.0898 & 0.0452 & 0.1295 & 0.1886 & 0.0061 \\
\hline 4 (TR) & 0.1479 & 0.1012 & 0.1149 & 0.2764 & 0.5069 & 0.0231 \\
\hline 5 (IT) & 0.1086 & 0.0689 & 0.0722 & 0.2419 & 0.5033 & 0.0123 \\
\hline
\end{tabular}

Table A4.2

Case 2: CPI inflation with interest rate smoothing

\begin{tabular}{c|c|c|c|c|c|c}
\hline \multirow{2}{*}{ Policy Rule } & \multicolumn{5}{|c|}{ Standard Deviation } & Welfare \\
\cline { 2 - 6 } & $\hat{\Pi}_{H, t}$ & $x_{t}$ & $R_{g, t}$ & $q_{g, t}$ & $\hat{\Psi}_{t}$ & \multirow{2}{*}{ loss } \\
\hline 1 (C) & 0.0859 & 0.1071 & 0.0435 & 0.1587 & 0.2299 & 0.0076 \\
\hline 2 (EX) & 0.1058 & 0.1202 & 0.0540 & 0.1948 & 0.3000 & 0.0115 \\
\hline 3 (PT) & 0.0724 & 0.0976 & 0.0372 & 0.1354 & 0.1883 & 0.0054 \\
\hline 4 (TR) & 0.1216 & 0.1297 & 0.0654 & 0.2348 & 0.3873 & 0.0152 \\
\hline 5 (IT) & 0.0868 & 0.1205 & 0.0210 & 0.2453 & 0.4546 & 0.0076 \\
\hline
\end{tabular}

A4.2 Simple policy rules in Case 3 where the monetary authority can access full information only with a one period lag

Tables A4.3 through A4.6 record the simulation results for our four separate subcases when the monetary authority uses the consumer price (CPI) measure of inflation in the case where the monetary authority can access relevant information with a one period lag. Compared to the use of domestic output prices in Table 4.13 through 4.16 , the CPI results in Tables A4.3 through A4.6 do not add much new information. The important 
exception is that it now doesn't appear to matter for policy performance whether the inflation rate or the inflation rate gap is targeted. In some cases, the use of the inflation rate gap improves monetary policy performance quite a bit in comparison with using just the inflation rate. This is especially the case for the inflation forecast Taylor rule, Rule 7.

Table A4.3

Case 3: Consumer prices used to measure inflation

\begin{tabular}{c|c|c|c|c|c|c}
\hline \multirow{2}{*}{ Policy Rule } & \multicolumn{5}{|c|}{ Standard Deviation } & \multirow{2}{*}{ Welfare loss } \\
\cline { 2 - 6 } & $\hat{\Pi}_{H, t}$ & $x_{t}$ & $R_{g, t}$ & $q_{g, t}$ & $\hat{\Psi}_{t}$ & \\
\hline $\mathbf{1}$ (C) & 0.0279 & 0.0476 & 0.0489 & 0.0747 & 0.1442 & $9.9807 \mathrm{e}-004$ \\
\hline 2 (EX) & 0.0229 & 0.0414 & 0.0494 & 0.0789 & 0.1639 & $7.4762 \mathrm{e}-004$ \\
\hline 3 (PT) & 0.0326 & 0.0535 & 0.0484 & 0.0729 & 0.1293 & 0.0013 \\
\hline 4 (TR) & 0.0186 & 0.0393 & 0.0440 & 0.0999 & 0.1922 & $5.2542 \mathrm{e}-004$ \\
\hline 5 (IT) & 0.0329 & 0.0528 & 0.0428 & 0.1067 & 0.1759 & 0.0013 \\
\hline 6 (SIF) & 0.0435 & 0.0626 & 0.0450 & 0.1126 & 0.1681 & 0.0021 \\
\hline 7 (IFTR) & 0.0227 & 0.0415 & 0.0452 & 0.0995 & 0.1860 & $7.0446 \mathrm{e}-004$ \\
\hline
\end{tabular}

Table A4.4

Case 3: CPI measure of inflation with interest rate smoothing

\begin{tabular}{c|c|c|c|c|c|c}
\hline \multirow{2}{*}{ Policy Rule } & \multicolumn{5}{|c|}{ Standard Deviation } & \multirow{2}{*}{ Welfare loss } \\
\cline { 2 - 6 } & $\hat{\Pi}_{H, t}$ & $x_{t}$ & $R_{g, t}$ & $q_{g, t}$ & $\hat{\Psi}_{t}$ & \\
\hline 1 (C) & 0.0293 & 0.0549 & 0.0467 & 0.1043 & 0.1873 & 0.0011 \\
\hline 2 (EX) & 0.0262 & 0.0511 & 0.0475 & 0.1110 & 0.2082 & $\mathbf{8 . 9 7 7 4 \mathrm { e } - 0 0 4}$ \\
\hline 3 (PT) & 0.0327 & 0.0588 & 0.0460 & 0.1003 & 0.1717 & 0.0013 \\
\hline 4 (TR) & 0.0317 & 0.0518 & 0.0526 & 0.1268 & 0.2346 & 0.0013 \\
\hline 5 (IT) & 0.0410 & 0.0641 & 0.0561 & 0.1295 & 0.2195 & 0.0020 \\
\hline 6 (SIF) & 0.0470 & 0.0681 & 0.0599 & 0.1321 & 0.2180 & 0.0025 \\
\hline 7 (IFTR) & 0.0351 & 0.0522 & 0.0577 & 0.1284 & 0.2367 & 0.0015 \\
\hline
\end{tabular}


Table A4.5

Case 3: The CPI measure of the inflation gap

\begin{tabular}{c|c|c|c|c|c|c}
\hline \multirow{2}{*}{ Policy Rule } & \multicolumn{5}{|c|}{ Standard Deviation } & Welfare loss \\
\cline { 2 - 6 } & $\hat{\Pi}_{H, t}$ & $x_{t}$ & $R_{g, t}$ & $q_{g, t}$ & $\hat{\Psi}_{t}$ & \\
\hline 1 (C) & 0.0289 & 0.0589 & 0.0567 & 0.0719 & 0.1357 & 0.0011 \\
\hline 2 (EX) & 0.0244 & 0.0524 & 0.0578 & 0.0752 & 0.1579 & $9.0060 \mathrm{e}-004$ \\
\hline 3 (PT) & 0.0336 & 0.0651 & 0.0563 & 0.0715 & 0.1196 & 0.0014 \\
\hline 4 (TR) & 0.0184 & 0.0496 & 0.0500 & 0.0936 & 0.1866 & $5.6990 \mathrm{e}-004$ \\
\hline 5 (IT) & 0.0303 & 0.0673 & 0.0453 & 0.0990 & 0.1595 & 0.0011 \\
\hline 6 (SIF) & 0.0323 & 0.0545 & 0.0425 & 0.1101 & 0.1802 & 0.0012 \\
\hline 7 (IFTR) & 0.0162 & 0.0378 & 0.0432 & 0.1033 & 0.2036 & $4.3591 \mathrm{le}-004$ \\
\hline
\end{tabular}

Table A4.6

Case 3: The CPI measure of the inflation gap and interest rate smoothing

\begin{tabular}{c|c|c|c|c|c|c}
\hline \multirow{2}{*}{$\begin{array}{c}\text { Policy } \\
\text { Rule }\end{array}$} & \multicolumn{5}{|c|}{ Standard Deviation } & \multirow{2}{*}{ Welfare loss } \\
\cline { 2 - 6 } & $\hat{\Pi}_{H, t}$ & $x_{t}$ & $R_{g, t}$ & $q_{g, t}$ & $\hat{\Psi}_{t}$ & \\
\hline 1 (C) & 0.0297 & 0.0578 & 0.0470 & 0.1007 & 0.1796 & 0.0011 \\
\hline 2 (EX) & 0.0263 & 0.0532 & 0.0478 & 0.1069 & 0.2011 & $9.0572 \mathrm{e}-004$ \\
\hline 3 (PT) & 0.0333 & 0.0622 & 0.0464 & 0.0973 & 0.1638 & 0.0013 \\
\hline 4 (TR) & 0.0306 & 0.0531 & 0.0511 & 0.1221 & 0.2272 & 0.0012 \\
\hline 5 (IT) & 0.0401 & 0.0666 & 0.0535 & 0.1243 & 0.2083 & 0.0019 \\
\hline 6 (SIF) & 0.0405 & 0.0629 & 0.0591 & 0.1372 & 0.2396 & 0.0020 \\
\hline 7 (IFTR) & 0.0325 & 0.0508 & 0.0547 & 0.1355 & 0.2564 & 0.0013 \\
\hline
\end{tabular}

A4. 3 Simple policy rules in Case 4 where the monetary authority must use steady state values

Tables A4.7 and A4.8 list the simulation results when the policy maker targets inflation measured in CPI prices in Case 4. 
Table A4.7

Case 4: The use of steady state values with a CPI inflation measure

\begin{tabular}{c|c|c|c|c|c|c}
\hline \multirow{2}{*}{$\begin{array}{c}\text { Policy } \\
\text { Rule }\end{array}$} & \multicolumn{5}{|c|}{ Standard Deviation } & Welfare loss \\
\cline { 2 - 6 } & $\hat{\Pi}_{H, t}$ & $x_{t}$ & $R_{g, t}$ & $q_{g, t}$ & $\hat{\Psi}_{t}$ & \\
\hline $\mathbf{1}$ (C) & 0.0881 & 0.1011 & 0.0669 & 0.1571 & 0.2368 & 0.0082 \\
\hline $\mathbf{2}$ (EX) & 0.1096 & 0.1110 & 0.0837 & 0.1988 & 0.3224 & 0.0127 \\
\hline 3 (PT) & 0.0739 & 0.0936 & 0.0578 & 0.1306 & 0.1854 & 0.0058 \\
\hline $\mathbf{4}$ (TR) & 0.1315 & 0.1113 & 0.1040 & 0.2494 & 0.4424 & 0.0183 \\
\hline 5 (IT) & 0.0881 & 0.0853 & 0.0621 & 0.2181 & 0.4392 & 0.0081 \\
\hline 6 (SIF) & 0.1211 & 0.1085 & 0.0665 & 0.2795 & 0.5578 & 0.0151 \\
\hline $\mathbf{7}$ (IF) & 0.1707 & 0.1343 & 0.1229 & 0.3228 & 0.5864 & 0.0306 \\
\hline
\end{tabular}

Table A4.8

Case 4: steady state values, a CPI inflation measure, and interest rate smoothing

\begin{tabular}{c|c|c|c|c|c|c}
\hline \multirow{2}{*}{$\begin{array}{c}\text { Policy } \\
\text { Rule }\end{array}$} & \multicolumn{5}{|c|}{ Standard Deviation } & Welfare loss \\
\cline { 2 - 6 } & $\hat{\Pi}_{H, t}$ & $x_{t}$ & $R_{g, t}$ & $q_{g, t}$ & $\hat{\Psi}_{t}$ & \\
\hline $\mathbf{1}$ (C) & 0.0849 & 0.1138 & 0.0527 & 0.1644 & 0.2334 & 0.0075 \\
\hline $\mathbf{2}$ (EX) & 0.1036 & 0.1288 & 0.0594 & 0.1964 & 0.2917 & 0.0111 \\
\hline $\mathbf{3}$ (PT) & 0.0720 & 0.1033 & 0.0488 & 0.1440 & 0.1998 & 0.0054 \\
\hline $\mathbf{4}$ (TR) & 0.1170 & 0.1426 & 0.0656 & 0.2321 & 0.3640 & 0.0141 \\
\hline $\mathbf{5}$ (IT) & 0.0908 & 0.1441 & 0.0421 & 0.2691 & 0.4772 & 0.0085 \\
\hline $\mathbf{6}$ (SIF) & 0.1160 & 0.1766 & 0.0430 & 0.3240 & 0.5686 & 0.0137 \\
\hline $\mathbf{7}$ (IF) & 0.1397 & 0.1672 & 0.0695 & 0.2755 & 0.4385 & 0.0200 \\
\hline
\end{tabular}




\section{Chapter 5}

\section{Fully Optimal Monetary Policy Rules for the Small Open Economy}

\subsection{The Mathematics of Optimal Policy Rules}

In most economic problems, agents (such as policy makers, individuals, or firms) are modeled as maximizing an objective function subject to various types of constraints. In an inter-temporal setting, such problems are often solved using optimal control theory, an approach developed by Pontryagin and his associates in the late 1950s (see Pontryagin 1962). Dynamic programming, developed by Bellman also in the late 1950 s, is an alternative popular method used to deal with this problem (Bellman, 1957). In many applications, however, Chow $(1975,1997)$ has shown that the Lagrangian method is computationally more efficient and more accurate than dynamic programming methods.

From this literature it is well known that in a linear-quadratic framework in which there are no forward-looking variables, optimal policy may be expressed as a timeinvariant feedback rule (a closed-loop form policy). For example, consider our problem in which a benevolent monetary authority chooses an optimal policy, $\hat{R}_{t}$, to maximize a time separable social objective function depending on the state variables $X_{t}$. This can be written formally as

$$
\max _{\left\{\hat{R}_{t} \hat{t}_{t=0}^{\mathfrak{W}}\right.} E_{0} \sum_{t=0}^{\infty}\left[X_{t}^{\prime} Q_{1} X_{t}+\hat{R}_{t}^{\prime} Q_{2} \hat{R}_{t}\right]
$$

subject to a set of equations that describe the dynamic evolution of the state variables,

$$
X_{t+1}=A X_{t}+B \hat{R}_{t}+V_{t+1} .
$$


Following the standard procedure for solving these types of dynamic optimization problems, ${ }^{71}$ the optimal policy can be rewritten as

$$
\hat{R}_{t}=-\left[Q_{2}+B^{\prime} K B\right]^{-1} B^{\prime} K A X_{t}
$$

where

$$
K=Q_{1}+A^{\prime}\left[K-K B\left(Q_{2}+B^{\prime} K B\right)^{-1} B^{\prime} K\right\rfloor A
$$

Here (5.4) is known as the matrix Riccati equation, and $K$ can be solved for iteratively.

The optimal policy described by this solution has the following properties (see Currie and Levine, 1993, pp.9). First, providing the future is not discounted too heavily, the optimal rule will stabilize the endogenous variables. Second, the optimal rule will be linear in the entire state vector. Third, the optimal rule will be independent of the initial state of the system. Fourth, when the uncertainty is introduced into the model, the optimal rule will remain the same as that in the deterministic problem and will be independent of the covariance matrix of the white noise disturbances. This is the certainty equivalence property. Lastly, the optimal rule will be time consistent. The optimal rule described at time $t>0$ is simply a continuation of the rule chosen at time $t=0$. Neither deterministically nor under uncertainty will the optimal rule depend upon the current state of the system.

In current economic analysis, models containing only backward-looking variables are increasingly rare. This is because future outcomes typically affect the incentives facing agents today. In relation to policy, this implies that rational economic agents can be expected to anticipate the consequences of current and future policy when choosing their behavior today and this in turn needs to be anticipated by policy makers. Hence in

\footnotetext{
${ }^{71}$ The standard procedures used to solve this dynamic optimization problem are described in Chow (1975, 1997) and in Bertesekas (1976).
} 
analyses where economic agents are assumed to be rational, economic behavior is now modeled as forward-looking. Since the early 1990s a number of new analytical frameworks have been developed on this basis, often referred as new Keynesian or new Classical approaches, both of which are consistent with optimizing behavior by private agents who form expectations of future events under rational expectations. The resulting models then incorporate forward-looking variables.

If we introduce rational expectations in relation to the future values of the state variables and hence allow the effects of forward-looking variables into equation (5.2), above, the model can now be expressed as

$$
A_{1} E_{t} X_{t+1}=A_{2} X_{t}+B_{1} \hat{R}_{t}+V_{t+1}
$$

Just as was the case for models with backward-looking variables, numerical solution methods have been developed to solve forward-looking rational expectations models. ${ }^{72}$ One important solution method has been proposed by Blanchard and Kahn (1980). Their key points are as follows: first, distinguish variables by whether they are predetermined or non-predetermined. This modifies (5.5) in the following way

$$
\left[\begin{array}{l}
X_{1, t+1} \\
E_{t} X_{2, t+1}
\end{array}\right]=A\left[\begin{array}{l}
X_{1, t} \\
X_{2, t}
\end{array}\right]+A_{1}^{-1} V_{t+1}
$$

where $X_{1, t}$ is a $n_{1} \times 1$ vector of variables predetermined at time $t, X_{2, t}$ is a $n_{2} \times 1$ variables non-predetermined at time $t$, and $A=A_{1}^{-1} A_{2}$. Next, $A$ is transformed into a Jordan canonical form, $A=C^{-1} J C$, where the diagonal elements of $J$, i.e., the eigenvalues of $A$, are ordered by their increasing absolute value. $J$ is then further

\footnotetext{
${ }^{72}$ For example, Calvo (1978), Blanchard and Kahn (1980), Kydland and Prescott (1982), Currie and Levine (1982, 1987, 1993), McCallum (1983, 1998), Backus and Driffill (1986), King and Watson $(1998,2002)$, Binder and Pesaran (1995, 1996), Klein (2000), Sims (2001), Uhlig (1999), Soderlind (1999), and Dennis (2004).
} 
decomposed into $J=\left[\begin{array}{cc}J_{1} & 0 \\ 0 & J_{2}\end{array}\right]$, where the partition in $J$ has all the eigenvalues that are on or inside the unit circle in $J_{1}$, a $\bar{n}_{1} \times \bar{n}_{1}$ matrix, and all eigenvalues that are outside the unit circle are in $J_{2}$, a $\bar{n}_{2} \times \bar{n}_{2}$ matrix. Third, define the auxiliary variables $\left[\begin{array}{l}Y_{1, t} \\ Y_{2, t}\end{array}\right]=C\left[\begin{array}{l}X_{1, t} \\ X_{2, t}\end{array}\right]$, and premultiplying both sides of (5.6) by $C$ and using $A=C^{-1} J C$, (5.6) then becomes

$$
\left[\begin{array}{l}
Y_{1, t+1} \\
E_{t} Y_{2, t+1}
\end{array}\right]=\left[\begin{array}{cc}
J_{1} & 0 \\
0 & J_{2}
\end{array}\right]\left[\begin{array}{l}
Y_{1, t} \\
Y_{2, t}
\end{array}\right]+C A_{1}^{-1} V_{t+1}
$$

Finally, (5.7) presents the following types of solutions. If $\bar{n}_{2}=n_{2}$, that is, if the number of eigenvalues of $A$ outside the unit circle is equal to the number of non-predetermined variables, then there exists a unique solution. Otherwise, (5.7) either has no solution $\left(\bar{n}_{2}>n_{2}\right)$ or has many solutions $\left(\bar{n}_{2}<n_{2}\right)$.

One limitation of Blanchard and Kahn's numerical solution method is that it requires the matrix $A_{1}$ to be invertible, which implies that static (intratemporal) equilibrium conditions should be excluded from the dynamic relationships, equation (5.5). however, Sims (2001), King and Watson (1998, 2002), and Klein (2000) have extended Blanchard and Kahn's numerical method (1980) to allow for $A_{1}$ singularities by using the complex generalized Schur form or $Q Z$ decomposition to find the solutions.

Blanchard and Kahn's numerical method and its extensions are based on matrix decomposition. Alternatively, McCallum (1983, 1998), Binder and Pesaran (1995, 1996), and Uhlig (1999) among others, use an undetermined coefficients approach to solve recursive stochastic linear systems. The general procedure under this method consists of 
the following steps. First, find all the necessary equations characterizing the equilibrium of the system. Next, log-linearize all these equations. Third, postulate a recursive equilibrium law of motion, which is to write all variables as linear functions of a vector of endogenous variables and exogenous variables. And finally solve it via the method of undetermined coefficients.

Mathematically speaking, solving the recursive equilibrium law of motion through the undetermined coefficients approach involves a matrix quadratic equation. Uhlig (1999) shows that this matrix quadratic equation can be solved by either turning it into a generalized eigenvalue and eigenvector problem or using the $Q Z$ decomposition. From this perspective, then, there is no essential difference between Blanchard and Kahn's method and the undetermined coefficients approach.

The rest of chapter is organized as follows. In section 5.2 I follow Soderlind (1999), who develops a number of useful methods and algorithms based on Blanchard and Kahn (1980), Sims (2001) and Klein (2000) to solve the rational expectations model and find the optimal policy rule under commitment. Section 5.3, the last section, offers my concluding remarks.

\subsection{A Fully Optimal Monetary Policy Rule under Commitment}

To find the fully optimal monetary policy rule under commitment, a monetary authority is assumed to choose its policy instrument to maximize a welfare function (or minimize the associated loss function) based on household utility, subject to the dynamic stochastic evolution of the underlying economy. In our analysis, that mathematical 
framework of the dynamic stochastic small open economy model was derived in Chapter

2. After some algebra manipulation the key equations can be rewritten as follows. ${ }^{73}$

The new Keynesian Phillips curve is

$$
\hat{\Pi}_{H, t}=P_{H 1} \hat{\Pi}_{H, t-1}+P_{H 2} E_{t} \hat{\Pi}_{H, t+1}+P_{H 3} x_{t}-P_{H 4} q_{g, t}+P_{H S} \hat{\Psi}_{t}
$$

Where $P_{H 1}=\frac{\delta}{1+\beta \delta}, P_{H 2}=\frac{\beta}{1+\beta \delta}, \quad P_{H 3}=\frac{\gamma(\mu+\sigma-\mu \alpha)}{1-\alpha}, \quad P_{H 4}=\frac{\gamma(\phi+\alpha-1)}{(1-\alpha)^{2}}, \quad$ and $P_{H S}=\frac{\gamma \alpha(\sigma \eta+\alpha-1)}{(1-\alpha)^{2}}$

The import goods price equation is

$$
\hat{\Pi}_{F, t}=P_{F 1} \hat{\Pi}_{F, t-1}+P_{F 2} E_{t} \hat{\Pi}_{F, t+1}+P_{F 3} \hat{\Psi}_{t}
$$

Where $P_{F 1}=\frac{\delta}{1+\beta \delta}, P_{F 2}=\frac{\beta}{1+\beta \delta}$, and $P_{F 3}=\frac{\theta-1}{\omega_{M}(1+\beta \delta)}$

The IS equation is

$$
\begin{aligned}
& x_{t}=E_{t} x_{t+1}-X_{1} R_{g, t}-X_{2} E_{t} \hat{\Pi}_{H, t+1}-X_{3} E_{t} \hat{\Pi}_{F, t+1}+X_{4} E_{t} q_{g, t+1} \\
& -X_{5} q_{g, t}+X_{6} \hat{A}_{t}+X_{7} \hat{\chi}_{1 t}-X_{8} \hat{Y}_{t}^{*}
\end{aligned}
$$

Where $X_{1}=\frac{1-\alpha}{\sigma}, X_{2}=\frac{1-\alpha+\alpha(\sigma \eta+\alpha-1)}{\sigma}, X_{3}=\frac{\alpha(\sigma \eta+\alpha-1)}{\sigma}$,

$$
\begin{aligned}
& X_{4}=\frac{\phi+\alpha-1-\alpha(\sigma \eta+\alpha-1)}{\sigma(1-\alpha)}, X_{5}=\frac{\phi+\alpha-1-\alpha(\sigma \eta+\alpha-1)}{\sigma(1-\alpha)}, X_{6}=\frac{\alpha(\sigma \eta+\alpha-1)(1+\mu)}{\phi \mu+\sigma} \\
& X_{6}=\left(1-\rho_{A}\right), X_{7}=\frac{\alpha(\sigma \eta+\alpha-1)}{\phi \mu+\sigma}\left(1-\rho_{1}\right), X_{8}=\frac{\alpha(\sigma \eta+\alpha-1)(\sigma+\mu)}{\phi \mu+\sigma}\left(1-\rho_{y}\right) .
\end{aligned}
$$

The real exchange rate equation is

\footnotetext{
${ }^{73}$ There are two versions of the model: One is expressed in terms of the domestic output price inflation, and another is written in terms of CPI inflation. Here we focus on the former version. In order to write the model in state-space form, some of equations have been modified. In addition, note that $q_{g, t}=\hat{q}_{t}-\hat{q}_{t}^{f}$, $R_{g, t}=\hat{R}_{t}-\hat{r}_{H, t}{ }^{f}$
} 


$$
\begin{aligned}
& Q_{1} q_{g, t}=Q_{2} E_{t} q_{g, t+1}+Q_{3}\left(x_{t}-E_{t} x_{t+1}\right)-Q_{4} \hat{\Pi}_{H, t+1}+Q_{5} \hat{\Pi}_{F, t+1} \\
& -Q_{6} N F A_{t}-Q_{7} \hat{\chi}_{2 t}-Q_{8} \hat{A}_{t}-Q_{9} \hat{\chi}_{1 t}+Q_{10} \hat{Y}_{t}^{*}
\end{aligned}
$$

Where $\quad Q_{1}=(\phi-\alpha \sigma \eta), \quad Q_{2}=(\phi-\alpha \sigma \eta), \quad Q_{3}=\sigma(1-\alpha), \quad Q_{4}=\alpha \sigma \eta(1-\alpha)$,

$$
\begin{aligned}
& Q_{5}=\alpha \sigma \eta(1-\alpha), \quad Q_{6}=b(1-\alpha)^{2}, \quad Q_{7}=(1-\alpha)^{2}, \quad Q_{8}=\frac{\alpha \sigma^{2} \eta(1-\alpha)(1+\mu)}{\phi \mu+\sigma}\left(1-\rho_{A}\right), \\
& Q_{9}=\frac{\alpha \sigma^{2} \eta(1-\alpha)}{\phi \mu+\sigma}\left(1-\rho_{1}\right), Q_{10}=\frac{\alpha \sigma^{2} \eta(1-\alpha)(\sigma+\mu)}{\phi \mu+\sigma}\left(1-\rho_{y}\right) .
\end{aligned}
$$

The net foreign asset position equation is

$$
N F A_{t}=N_{1} N F A_{t-1}-N_{2} x_{t}+N_{3} q_{g t}-N_{4} \hat{\Psi}_{t}+N_{5} \hat{A}_{t}+N_{6} \hat{\chi}_{1 t}-N_{7} \hat{Y}_{t}
$$

Where $\quad N_{1}=\frac{1}{\beta}, \quad N_{2}=\frac{\alpha}{\beta(1-\alpha)}, \quad N_{3}=\frac{\alpha[\eta(2-\alpha)+\alpha-1]}{\beta(1-\alpha)^{2}}, \quad N_{4}=\frac{\alpha(\eta+\alpha-1)}{\beta(1-\alpha)^{2}}$,

$$
\begin{aligned}
& N_{5}=\frac{\alpha \sigma[\eta(2-\alpha)+\alpha-1](1+\mu)-\alpha \phi(1+\mu)}{\beta(1-\alpha)(\phi \mu+\sigma)}, N_{6}=\frac{\alpha \sigma[\eta(2-\alpha)+\alpha-1]-\alpha \phi^{2}(1+\mu)}{\beta(1-\alpha)(\phi \mu+\sigma)}, \\
& N_{7}=\left[\frac{\alpha \sigma[\eta(2-\alpha)+\alpha-1](\sigma+\mu)+\alpha \sigma(1-\phi)}{\beta(1-\alpha)(\phi \mu+\sigma)}-\frac{\alpha}{\beta(1-\alpha)}\right]
\end{aligned}
$$

The law of one price gap equation is

$$
\begin{aligned}
& \hat{\Psi}_{t}=\hat{\Psi}_{t-1}+(1-\alpha) \hat{\Pi}_{H, t}-(1-\alpha) \hat{\Pi}_{F, t}+q_{g, t}-q_{g, t-1} \\
& +H_{1}\left(\hat{A}_{t}-\hat{A}_{t-1}\right)+H_{2}\left(\hat{\chi}_{1 t}-\hat{\chi}_{1 t-1}\right)-H_{3}\left(\hat{Y}_{t}^{*}-\hat{Y}_{t-1}{ }^{*}\right)
\end{aligned}
$$

where $H_{1}=\frac{\sigma(1-\alpha)(1+\mu)}{\phi \mu+\sigma}, H_{2}=\frac{\sigma(1-\alpha)}{\phi \mu+\sigma}, H_{3}=\frac{\sigma(1-\alpha)(\sigma+\mu)}{\phi \mu+\sigma}$.

Equations (5.8) through (5.13) plus four shocks can be written compactly as

$$
A_{1}\left[\begin{array}{l}
X_{1, t+1} \\
E_{t} X_{2, t+1}
\end{array}\right]=A_{2}\left[\begin{array}{l}
X_{1, t} \\
X_{2, t}
\end{array}\right]+B R_{g, t}+\left[\begin{array}{l}
\varepsilon_{t+1} \\
0_{2}
\end{array}\right]
$$

where $X_{1, t}$ is an $12 \times 1$ column vector of predetermined variables defined as 


$$
X_{1, t}=\left[\hat{A}_{t}, \hat{\chi}_{1 t}, \hat{\chi}_{2 t}, \hat{Y}_{t}^{*}, \hat{A}_{t-1}, \hat{\chi}_{1 t-1}, \hat{Y}_{t-1}^{*}, \hat{\Pi}_{H, t-1}, \hat{\Pi}_{F, t-1}, q_{g, t-1}, N F A_{t-1}, \hat{\psi}_{t-1}\right]^{\prime}
$$

Where the $X_{1,0}$ are all givens. $X_{2, t}$, on the other hand, is an $4 \times 1$ column vector of nonpredetermined variables defined as

$$
X_{2, t}=\left[\hat{\Pi}_{H, t}, \hat{\Pi}_{F, t}, x_{t}, q_{g, t}\right]^{\prime} .
$$

$R_{g, t}$, in (5.14) is the gap between the short-term interest rate and the Wicksellian natural rate and is the policy instrument. $\varepsilon_{t+1}$ is an $12 \times 1$ column vector of innovations to the economic system and $\mathrm{O}_{2}$ is an $4 \times 1$ zero column vector. Lastly, $A_{1}$ and $A_{2}$ are $16 \times 16$ matrices, and $B$ an $16 \times 1$ column vector of the structural parameters, all of which are assumed to be constants.

Note that (5.14) can be further rewritten compactly as

$$
A_{1} X_{t+1}=A_{2} X_{t}+B R_{g, t}+V_{t+1}
$$

where $X_{t+1}=\left[X_{1, t+1}, E_{t} X_{2, t+1}\right]^{\prime}, \quad X_{t}=\left[X_{1, t}, X_{2, t}\right]^{\prime}$, and $V_{t+1}=\left[\varepsilon_{t+1}, 0_{4 \times 1}\right]^{\prime}$, where The monetary authority's objective function derived in Chapter 3 is,

$$
E_{0} \sum_{t=0}^{\infty} \beta^{t} U_{t} \approx-\Omega E_{0} \sum_{t=0}^{\infty} \beta^{t}\left\{\begin{array}{l}
\hat{\Pi}_{H, t}{ }^{2}+\phi_{1} x_{t}{ }^{2}+\phi_{2} q_{g, t}{ }^{2} \\
+\phi_{3} \hat{\Psi}_{t}{ }^{2}+\phi_{4} R_{g, t}{ }^{2}+\text { c.p.t. }+ \text { li.p. p. }
\end{array}\right\}+\text { t.i.p. }+o\left(\|\ddot{\xi}\|^{3}\right),
$$

which can be equivalently rewritten as a loss function

$$
W L_{0}=E_{0} \sum_{t=0}^{\infty} \beta^{t}\left(X_{t}{ }^{\prime} Q_{1} X_{t}+R_{g, t}{ }^{\prime} Q_{2} R_{g, t}\right),
$$

where $Q_{1}$ is a $16 \times 16$ matrix, and $Q_{2}=\phi_{4}$.

To find the optimal policy, then, the monetary authority needs to set up and solve the following Lagrangian function, 


$$
L=E_{0}\left\{\sum_{t=0}^{\infty}\left[\beta^{t}\left(X_{t}^{\prime} Q_{1} X_{t}+R_{g, t}{ }^{\prime} Q_{2} R_{g, t}\right)-2 \beta^{t+1} \lambda_{t+1}{ }^{\prime}\left(A_{1} X_{t+1}-A_{2} X_{t}-B R_{g, t}-V_{t+1}\right)\right]\right\},
$$

where $\lambda_{t+1}=\left[\lambda_{1, t+1}, E_{t} \lambda_{2, t+1}\right]^{1}$, and $\lambda_{1, t+1}$ is a $12 \times 1$ column vector, and $\lambda_{2, t+1}$ is a $4 \times 1$ column vector. After simple algebraic manipulation, the first-order conditions with respect to $\lambda_{t+1}, R_{g, t}$, and $X_{t}$, respectively, can be expressed as

$$
\begin{aligned}
& A_{1} X_{t+1}=A_{2} X_{t}+B R_{g, t}+V_{t+1} \\
& \beta B^{\prime} \lambda_{t+1}=-Q_{2} R_{g, t} \\
& \beta A_{2}{ }^{\prime} \lambda_{t+1}=-Q_{1} X_{t}+A_{1}{ }^{\prime} \lambda_{t}
\end{aligned}
$$

where $\lambda_{t}=\left[\lambda_{1, t}, \lambda_{2, t}\right]^{\prime}$. Writing equations (5.18), (5.19), and (5.20) in the state-space form we obtain

$$
\left[\begin{array}{ccc}
A_{1} & 0_{16 \times 1} & 0_{16 \times 16} \\
0_{1 \times 16} & 0_{1 \times 1} & \beta B^{\prime} \\
0_{16 \times 16} & 0_{16 \times 1} & \beta A_{2}{ }^{\prime}
\end{array}\right]\left[\begin{array}{c}
X_{t+1} \\
R_{g, t+1} \\
\lambda_{t+1}
\end{array}\right]=\left[\begin{array}{ccc}
A_{2} & B & 0_{16 \times 16} \\
0_{1 \times 16} & -Q_{2} & 0_{1 \times 16} \\
-Q_{1} & 0_{16 \times 1} & A_{1}{ }^{\prime}
\end{array}\right]\left[\begin{array}{c}
X_{t} \\
R_{g, t} \\
\lambda_{t}
\end{array}\right]+\left[\begin{array}{c}
V_{t+1} \\
0_{1 \times 1} \\
0_{16 \times 1}
\end{array}\right]
$$

The following is simply an application of Soderlind's (1999) algorithm. First, taking expectations of (5.21) conditional on the information set in $t$, then using definitions of $X_{t}$ and $\lambda_{t}$ to rearrange variables in the order $\left[X_{1, t}, \lambda_{2 t}, X_{2, t}, R_{g, t}, \lambda_{1, t}\right]$. The coefficient matrices are reordered accordingly. The reason for this rearrangement is that the initial conditions for predetermined variables, $X_{1, a}$ are known by assumption while the non-predetermined variables, $X_{2, k}$, can be chosen freely in the initial period. However, the costate variables associated with these non-predetermined variables (i.e., the shadow prices or Lagrangian multipliers) in the initial period, $\lambda_{2,0}$, must be equal to 
zero. Hence the $\lambda_{2, t}$ are known, while costate variables associated with predetermined variables, $\lambda_{1, t}$, are unknowns (see Currie and Levine, 1993, pp.102).

If we define $k_{t}=\left[X_{1, t}, \lambda_{2, t}\right]^{\prime}$ and $h_{t}=\left[X_{2, t}, R_{g, t}, \lambda_{1, t}\right]$, we can then write (5.21) as

$$
G E_{t}\left[\begin{array}{l}
k_{t+1} \\
h_{t+1}
\end{array}\right]=D\left[\begin{array}{l}
k_{t} \\
h_{t}
\end{array}\right]
$$

where new matrices $G$ and $D$ are obtained by rearranging coefficient matrices in the order $\left[X_{1, t}, \lambda_{2 t}, X_{2, t}, R_{g, t}, \lambda_{1, t}\right]$ 'based on (5.21). Unfortunately, the matrix $G$ is singular, hence the algorithm proposed in Blanchard and Kahn (1980) cannot be used here. However, Sims (2001) and Klein (2000) show how the generalized Schur decomposition can be applied in this situation. Following the standard Schur decomposition procedure (see Golub and Van Loan, 1989), we obtain

$$
G=Q S Z^{H} \text { and } D=Q T Z^{H}
$$

where $S$ and $T$ are upper triangular matrices, $Q$ and $Z$ are unitary, that is, $Q^{H} Q=Z^{H} Z=I, Q^{H}$ is the transpose of the complex conjugate of $Q$, and $Z^{H}$ is the transpose of the complex conjugate of $Z$. Applying (5.23) in (5.22) yields

$$
Q S Z^{H} E_{t}\left[\begin{array}{l}
k_{t+1} \\
h_{t+1}
\end{array}\right]=Q T Z^{H}\left[\begin{array}{l}
k_{t} \\
h_{t}
\end{array}\right]
$$

Consider the transformation

$$
\left[\begin{array}{l}
s_{t} \\
u_{t}
\end{array}\right]=Z^{H}\left[\begin{array}{l}
k_{t} \\
h_{t}
\end{array}\right]
$$

Using (5.25) in (5.24) and then premultiplying both sides of the result of (5.24) gives

$$
S E_{t}\left[\begin{array}{c}
s_{t+1} \\
u_{t+1}
\end{array}\right]=T\left[\begin{array}{l}
s_{t} \\
u_{t}
\end{array}\right]
$$


This in turn can be written as

$$
\left[\begin{array}{cc}
S_{s s} & S_{s u} \\
0 & S_{u u}
\end{array}\right] E_{t}\left[\begin{array}{l}
s_{t+1} \\
u_{t+1}
\end{array}\right]=\left[\begin{array}{cc}
T_{s s} & T_{s u} \\
0 & T_{u u}
\end{array}\right]\left[\begin{array}{l}
s_{t} \\
u_{t}
\end{array}\right]
$$

Assuming that $T_{u u}$ and $S_{u u}$ are invertible, the lower block of (5.26) can be solved iteratively forward for $u_{t}$ to give

$$
\boldsymbol{u}_{t}=T_{u u}^{-1} S_{u u} \boldsymbol{u}_{t+1}=\left[T_{u u}^{-1} S_{u u}\right]^{\infty} \boldsymbol{u}_{t+\infty}
$$

By construction, the eigenvalues of the matrix $S_{u u}^{-1} T_{u u}$ are all greater than one.

It can be further shown that the eigenvalues of the inverse of matrix $S_{u u}{ }^{-1} T_{u u}$, that is, $T_{u u}^{-1} S_{u u}$, must be less than one (see Simon and Blume, 1994, pp.585). Furthermore, the $33 \times 33$ matrix $T_{u u}{ }^{-1} S_{u u}$ is diagonalizable because $T_{u u}{ }^{-1} S_{u u}$ has a set of 33 linearly independent eigenvectors. This means we can write $T_{u u}{ }^{-1} S_{u u}$ as

$$
P^{-1}\left[T_{u u}^{-1} S_{u u}\right] P=\operatorname{diag}\left[d_{1}, d_{2}, \ldots d_{i}\right]
$$

where $P$ is the eigenvector matrix, $d_{1}, d_{2}$, and $d_{i}$ are eigenvalues of the matrix $T_{u u}^{-1} S_{u u} \cdot(5.28)$ can be written as

$$
P^{-1}\left[T_{u u}^{-1} S_{u u}\right]^{\infty} P=\operatorname{diag}\left[d_{1}^{\infty}, d_{2}^{\infty}, \ldots d_{i}^{\infty}\right]
$$

Since by construction the eigenvalues $d_{i}<1$, where $i=33,\left[T_{u u}{ }^{-1} S_{u u}\right]^{\infty}$ is approaching zero. Thus from (5.27) we have $u_{t}=0$. Intuitively when the model involves both the backward-looking and forward-looking variables, to avoid having the system explode, the forward looking variables must jump to the unique non-explosive solution.

Given that $u_{t}=0$ and $S_{s s}$ is invertible, solving the upper block of (5.26) for $s_{t+1}$ yields 


$$
E_{t} s_{t+1}=S_{s s}^{-1} T_{s s} s_{t}
$$

Now turn back to (5.25), premultiply both sides of (5.25) by $Z$, and then partition $Z$ conformably with $y_{t}, u_{t}$, to obtain

$$
\left[\begin{array}{l}
k_{t} \\
h_{t}
\end{array}\right]=\left[\begin{array}{ll}
Z_{k s} & Z_{k u} \\
Z_{h s} & Z_{h u}
\end{array}\right]\left[\begin{array}{l}
s_{t} \\
u_{t}
\end{array}\right]=\left[\begin{array}{l}
Z_{k s} \\
Z_{h s}
\end{array}\right] s_{t}
$$

where the second equality has used the condition $u_{t}=0$. Solving $k_{t}$ from (5.31) we obtain

$$
k_{t}=Z_{k s} s_{t}
$$

From (5.14) we have $X_{1, t+1}-E_{t} X_{1, t+1}=\varepsilon_{t+1}$, and Backus and Driffill (1986) show that $\lambda_{2, t+1}-E_{t} \lambda_{2, t+1}=0_{2}$. Combining these two expressions gives

$$
\left[\begin{array}{l}
X_{1, t+1} \\
\lambda_{2, t+1}
\end{array}\right]-E_{t}\left[\begin{array}{l}
X_{1, t+1} \\
\lambda_{2, t+1}
\end{array}\right]=\left[\begin{array}{l}
\varepsilon_{t+1} \\
0_{2}
\end{array}\right],
$$

Using the definition $k_{t}$ and (5.32), (5.33) can be expressed as

$$
Z_{k s}\left(s_{t+1}-E_{t} s_{t+1}\right)=\left[\begin{array}{l}
\varepsilon_{t+1} \\
0_{2}
\end{array}\right]
$$

Applying (5.30) in (5.34) gives

$$
Z_{k s} s_{t+1}=Z_{k s} s_{s s}^{-1} T_{s s} s_{t}+\left[\begin{array}{l}
\varepsilon_{t+1} \\
0_{2}
\end{array}\right] \text {. }
$$

Assume that $Z_{k s}$ is invertible, we can rewrite (5.32) to be $s_{t}=Z_{k s}{ }^{-1} k_{t}$. Using it in (5.35) we have

$$
k_{t+1}=Z_{k s} S_{s s}^{-1} T_{s s} Z_{k s}^{-1} k_{t}+\left[\begin{array}{l}
\varepsilon_{t+1} \\
0_{2}
\end{array}\right]
$$


Using the definition $k_{t}$ again in (5.36) we obtain

$$
\left[\begin{array}{l}
X_{1, t+1} \\
\lambda_{2, t+1}
\end{array}\right]=Z_{k s} S_{s s}^{-1} T_{s s} Z_{k s}^{-1}\left[\begin{array}{l}
X_{1, t} \\
\lambda_{2, t}
\end{array}\right]+\left[\begin{array}{l}
\varepsilon_{t+1} \\
0_{2}
\end{array}\right] .
$$

Solving $h_{t}$ from (5.31) we have $h_{t}=Z_{h s} s_{t}$, and using (5.32) in it yields

$$
h_{t}=Z_{h s} Z_{k s}^{-1} k_{t}
$$

Finally, applying the definitions of $k_{t}$ and $h_{t}$ in (5.38) we obtain

$$
\left[\begin{array}{l}
X_{2, t} \\
R_{g, t} \\
\lambda_{1, t}
\end{array}\right]=Z_{h s} Z_{k s}^{-1}\left[\begin{array}{l}
X_{1, t} \\
\lambda_{2, t}
\end{array}\right]
$$

The solution to the model described by equations (5.15) and (5.16) is then given by equations (5.37) and (5.39), which determine how the economy in question evolves in the equilibrium. To obtain the fully optimal policy under commitment, let us first define $C=Z_{h s} Z_{k s}^{-1}$, and partition $C$ conformably with $X_{2, t}, R_{g, t}, \lambda_{1, t}, X_{1, t}$ and $\lambda_{2, t}$. We can then write (5.39) as

$$
\left[\begin{array}{l}
X_{2, t} \\
R_{g, t} \\
\lambda_{1, t}
\end{array}\right]=\left[\begin{array}{ll}
C_{11} & C_{12} \\
C_{21} & C_{22} \\
C_{31} & C_{32}
\end{array}\right]\left[\begin{array}{l}
X_{1, t} \\
\lambda_{2, t}
\end{array}\right]
$$

where $C_{11}$ is a $4 \times 12$ matrix, $C_{12}$, a $4 \times 4$ matrix, $C_{21}, 1 \times 12$ row vector, $C_{22}$, a $1 \times 4$ row vector, $C_{31}$, a $12 \times 12$ matrix, and $C_{32}$, a $12 \times 4$ matrix. From (5.40) we find the fully optimal policy for $R_{g, t}$, which is a function of the predetermined variables, $X_{1, t}$ and costate variables associated with the non-predetermined variables, $\lambda_{2, t}$. Specifically,

$$
R_{g, t}=C_{21} X_{1, t}+C_{22} \lambda_{2, t} .
$$


where $^{74}$

$$
\begin{gathered}
C_{21}=[-0.0353,-0.0149,-0.0195,0.0965,0.0227, \\
0.0057,-0.0238,0.04410 .0018,0.0397,0.0015,-0.0397] \\
\text { and } C_{22}=[-0.4602,-0.0080,-3.7786,0-0213] .
\end{gathered}
$$

The welfare loss incurred when the monetary authority sets its policy following (5.41) can be calculated by applying (5.37) and (5.40) into (5.16). From (5.40) we obtain

$$
\left.\left[X_{2, t}\right]=\left[\begin{array}{ll}
C_{11} & C_{12}
\end{array}\right] \begin{array}{l}
X_{1, t} \\
\lambda_{2, t}
\end{array}\right]
$$

Applying (5.42) in $X_{t}=\left[X_{1, t}{ }^{\prime}, X_{2, t}{ }^{\prime}\right]^{\prime}$ gives

$$
X_{t}=\left[\begin{array}{l}
X_{1, t} \\
X_{2, t}
\end{array}\right]=\left[\begin{array}{cc}
I_{12 \times 12} & 0_{12 \times 4} \\
C_{11} & C_{12}
\end{array}\right]\left[\begin{array}{l}
X_{1, t} \\
\lambda_{2, t}
\end{array}\right]
$$

And (5.41) can be rewritten to be

$$
\left.R_{g, t}=\left[\begin{array}{ll}
C_{21} & C_{22}
\end{array}\right] \begin{array}{l}
X_{1 t} \\
\lambda_{2 t}
\end{array}\right]
$$

Then using (5.43) and (5.44) in (5.16) yields

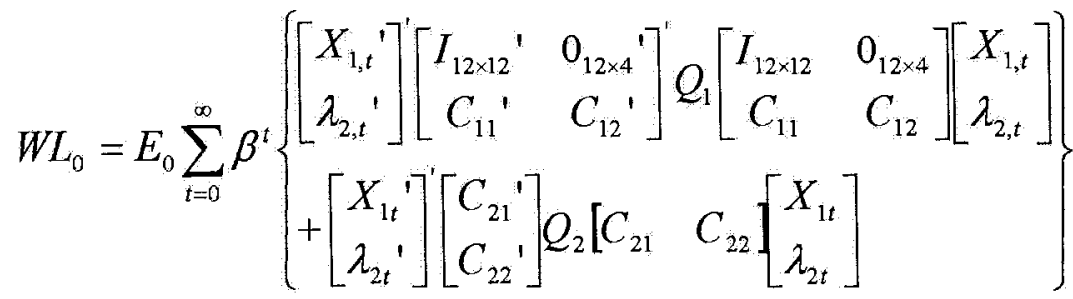

$$
\begin{aligned}
& =E_{0} \sum_{t=0}^{\infty} \beta^{t}\left[\begin{array}{l}
X_{1, t}{ }^{\prime} \\
\lambda_{2, t}{ }^{\prime}
\end{array}\right]^{\prime} N\left[\begin{array}{l}
X_{1 t} \\
\lambda_{2 t}
\end{array}\right]
\end{aligned}
$$

where $N=\left[\begin{array}{cc}I_{12 \times 12}{ }^{\prime} & 0_{12 \times 4}{ }^{\prime} \\ C_{11}{ }^{\prime} & C_{12}^{\prime}\end{array}\right]^{\prime} Q_{1}\left[\begin{array}{cc}I_{12 \times 12} & 0_{12 \times 4} \\ C_{11} & C_{12}\end{array}\right]+\left[\begin{array}{l}C_{21}{ }^{\prime} \\ C_{22}{ }^{\prime}\end{array}\right] Q_{2}\left[\begin{array}{ll}C_{21} & C_{22}\end{array}\right]$

\footnotetext{
${ }^{74}$ Note that $C_{21}$ and $C_{22}$ are calculated by using the Matlab program provided by Paul Soderlind.
} 
For convenience and simplicity we rewrite (5.37) as

$$
x_{1, t+1}=M x_{1, t}+V_{t+1}
$$

where $x_{1, t+1}=\left[X_{1, t+1}{ }^{1}, \lambda_{2, t+1}{ }^{\prime}\right]$ and $M=Z_{k s} S_{s s}{ }^{-1} T_{s s} Z_{k s}{ }^{-1}$. Substituting (5.46) into (5.45)

first and then expand the result of $(5.45)$, we have

$$
\begin{aligned}
& W L_{0}=E_{0}\left[x_{10}{ }^{\prime} N x_{10}+\beta x_{11}{ }^{\prime} N x_{11}+\beta^{2} x_{12}{ }^{\prime} N x_{12}+\cdots+\beta^{\infty} x_{100}{ }^{\prime} N x_{100}\right] \\
& =E_{0}\left[x_{10}{ }^{\prime} N x_{10}+\beta\left(M x_{10}+V_{1}\right)^{\prime} N\left(M x_{10}+V_{1}\right)\right. \\
& +\beta^{2}\left(M^{2} x_{10}+M V_{1}+V_{2}\right)^{\prime} N\left(M^{2} x_{10}+M V_{1}+V_{2}\right) \\
& \left.+\beta^{3}\left(M^{3} x_{10}+M^{2} V_{1}+M V_{1}+V_{3}\right)^{\prime} N\left(M^{3} x_{10}+M^{2} V_{1}+M V_{1}+V_{3}\right)+\cdots\right] \\
& =E_{0}\left[x_{10}{ }^{\prime} N x_{10}+\beta\left(x_{10}{ }^{\prime} M^{\prime} N M x_{10}+x_{10}{ }^{\prime} M^{\prime} N V_{1}+V_{1}^{\prime} N M x_{10}+V_{1}^{\prime} N V_{1}\right)\right. \\
& +\beta^{2}\left(x_{10}{ }^{\prime} M^{\prime 2} N M^{2} x_{10}+V_{1}^{\prime} M^{\prime} N M^{2} x_{10}+V_{2}^{\prime} N M^{2} x_{10}+x_{10}{ }^{\prime} M^{\prime 2} N M V_{1}\right. \\
& \left.+V_{1}^{\prime} M^{\prime} N V_{1}+V_{2}{ }^{\prime} N M V_{1}+x_{10}{ }^{\prime} M^{\prime 2} N V_{2}+V_{1}^{\prime} M^{\prime} N V_{2}+V_{2}^{\prime} N V_{2}\right) \\
& +\beta^{3}\left(x_{10}{ }^{\prime} M^{\prime 3} N M^{3} x_{10}+V_{1}^{\prime} M^{\prime 2} N M^{3} x_{10}+V_{2}{ }^{\prime} M^{\prime} N M^{3} x_{10}+V_{3}{ }^{\prime} N M^{3} x_{10}\right. \\
& +x_{10}{ }^{\prime} M^{\prime 3} N M^{2} V_{1}+V_{1}^{\prime} M^{\prime 2} N M^{2} V_{1}+V_{2}^{\prime} M^{\prime} N M^{2} V_{1}+V_{3}^{\prime} N M^{2} V_{1} \\
& +x_{10}{ }^{\prime} M^{\prime 3} N M V_{2}+V_{1}^{\prime} M^{\prime 2} N M V_{2}+V_{2}{ }^{\prime} M^{\prime} N V_{2}+V_{3}^{\prime} N M V_{2} \\
& \left.\left.+x_{10}{ }^{\prime} M^{\prime 3} N V_{3}+V_{1}^{\prime} M^{\prime 2} N V_{3}+V_{2}^{\prime} M^{\prime} N V_{3}+V_{3}^{\prime} N V_{3}\right)+\cdots\right]
\end{aligned}
$$

By assumption and construction, $E_{t}\left(V_{t+1}\right)=0$, and $\operatorname{Cov}\left(V_{t+i} V_{t+j}\right)=0$ for $i \neq j$. Taking expectations on (5.48) we obtain

$$
\begin{aligned}
& W L_{0}=x_{10}{ }^{\prime} N x_{10}+\beta\left(x_{10}{ }^{\prime} M^{\prime} N M x_{10}+E_{0} V_{1}{ }^{\prime} N V_{1}\right) \\
& +\beta^{2}\left(x_{10}{ }^{\prime} M^{\prime 2} N M^{2} x_{10}+E_{0} V_{1}^{\prime} M^{\prime} N M V_{1}+E_{0} V_{2}{ }^{\prime} N V_{2}\right) \\
& \left.+\beta^{3}\left(x_{10}{ }^{\prime} M^{\prime 3} N M^{3} x_{10}+E_{0} V_{1}^{\prime} M^{\prime 2} N M^{2} V_{1}+E_{0} V_{2}{ }^{\prime} M^{\prime} N M V_{2}+E_{0} V_{3}{ }^{\prime} N V_{3}\right)+\cdots\right]
\end{aligned}
$$

Collecting and rearranging terms it can be written as

$$
\begin{aligned}
& W L_{0}=x_{10} \cdot\left(\sum_{i=0}^{\infty} \beta^{i} M^{\prime i} N M^{i}\right) x_{10}+\beta E_{0} V_{1}^{\prime}\left(\sum_{i=0}^{\infty} \beta^{i} M^{\prime i} N M^{i}\right) V_{1} \\
& +\beta^{2} E_{0} V_{2}^{\prime}\left(\sum_{i=0}^{\infty} \beta^{i} M^{i} N M^{i}\right) V_{2}+\beta^{3} E_{0} V_{3}^{\prime}\left(\sum_{i=0}^{\infty} \beta^{i} M^{\prime^{i}} N M^{i}\right) V_{3}+\cdots
\end{aligned}
$$

Let $V=\sum_{i=0}^{\infty} \beta^{i} M^{i t} N M^{i}$, then (5.49) becomes 


$$
W L_{0}=x_{10}{ }^{\prime} V x_{10}+\beta E_{0} V_{1}^{\prime} V V_{1}+\beta^{2} E_{0} V_{2}{ }^{\prime} V V_{2}+\beta^{3} E_{0} V_{3}^{\prime} V V_{3}+\cdots
$$

Since $0<\beta<1$, we have

$$
W L_{0}=x_{10}^{\prime} V x_{10}+\frac{\beta}{1-\beta} \operatorname{tr}(V \Sigma)
$$

where $\operatorname{tr}$ denotes trace, and $\sum$ is a $16 \times 16$ variance matrix. ${ }^{75}$ For our problem, the resulting welfare loss under the fully optimal policy (5.41) under commitment is $0.1508^{76}$

\subsection{Concluding Remarks}

In a backward-looking framework, optimal monetary policies are found conventionally using techniques of optimal control, however when forward-looking variables are involved, newer numerical algorithms, such as those of Blanchard and Kahn (1980) should be employed. In such cases, the fully optimal monetary policy under commitment will be a function of all the predetermined variables and associated costate variables. While the fully optimal rule delivers the maximum welfare gain from policy, it

${ }^{75} V$ is found using the following steps. First, using the fixed-point theorem we have

$$
\beta M^{\prime} V M=\sum_{i=1}^{\infty} \beta^{i} M^{i} N M^{i}=\sum_{i=0}^{\infty} \beta^{i} M^{i} N M^{i}-\beta^{0} M^{\prime 0} N M^{0}=V-N,
$$

this gives us $V=N+\beta M^{\prime} V M$. Next, using vec operator to get $v e c(V)$

$\operatorname{vec}(V)=\operatorname{vec}(N)+\operatorname{vec}\left(\beta M^{\prime} V M\right)=\operatorname{vec}(N)+\left(M^{\prime} \otimes \beta M^{\prime}\right) \operatorname{vec}(V)$, so that $\operatorname{vec}(V)=\operatorname{vec}(N) /\left[I-\left(M^{\prime} \otimes \beta M^{\prime}\right)\right]$. Finally, reshape $\operatorname{vec}(V)$ to obtain matrix $V$.

${ }^{76}$ The welfare loss obtained here is not directly comparable to the results obtained in chapter 4 . First, the computation packages used are different. Second, the welfare loss calculated here is expected total loss, while Chapter 4 calculates only current period welfare loss. Third, for technical reasons, the actual welfare loss function used here is $W L_{0}=E_{0} \sum_{t=0}^{\infty} \beta^{t}\left\{\hat{\Pi}_{H, t}{ }^{2}+\phi_{1} x_{t}{ }^{2}+\phi_{2} q_{g, t}{ }^{2}+\phi_{3} \hat{\Psi}_{t-1}{ }^{2}+\phi_{4} R_{g, t}{ }^{2}\right\}$, which is slightly different from the objective function derived in Chapter 3 and used in Chapter 4. 
is also very difficult to apply in practice. This is because the complexity of the economic system makes acquiring all the relevant information needed impractical. Thus while simple optimal rule cannot minimize welfare losses compared to fully optimal rules when all relevant information is available, its absence may allow simple optimal rules to perform better in practice. That is, simple optimal rules are valued for their simplicity and transparency, and for the fact that they economize on information. This combination has made their use both practical and attractive. The optimal simple policy rules are investigated in Chapter 6. 


\section{Chapter 6}

\section{The Role of Openness Considerations in Optimal Simple Monetary Policy Rules}

\subsection{Optimal Simple Rules}

In principle it is possible for a monetary authority that can feasibly pre-commit to calculate the fully optimal policy rule given in (5.41). However, in practice the fully optimal rule is too complicated to be implemented economically. Three reasons for this stand out. First of all, to implement the fully optimal rule the monetary authority must be aware of all of the relevant variables in an economic system and distinguish between those that are predetermined and those that are not (since the optimal policy responds only to predetermined variables). Second, given the ability to distinguish all the relevant predetermined variables, policy maker must identify their actual values. In our case there are 16 predetermined variables, which implies that the policy maker needs to identify all four current period shocks, three shocks with one period lag, five endogenous variables with one period lag, and four costate variables. The shocks themselves are not directly observable and the costate variables are purely conceptual, introduced by the policy maker in order to solve the minimization problem and hence are unobservable as well. To extract the relevant information required to identify these variables requires a huge amount of knowledge of the operation of the economic system, making it virtually impossible to identify all relevant information accurately. Finally, communication becomes critical when the monetary authority must maintain the commitment needed to 
implement fully optimal policy. To both create and maintain credibility in the eyes of the public, the monetary authority needs to provide accurate timely information about the economy at large and its likely evolution through time. Provision of reliability needed for credibility becomes difficult when the policy maker has difficulty estimating the necessary but unobservable present and future variables.

It follows that in a world where information is costly, optimal simple rules may be efficient and this, in turn, allows us to use the convenience of their calculation and application in practice ${ }^{77}$ Assume then that the monetary authority can precommit to a simple policy rule of the form $\hat{R}_{t}=-F X_{t}$, where $\hat{R}_{t}$ is the policy instrument, $F$ is a $1 \times n$ row vector constrained to be sparse in some specified way which depends upon the policy maker's preferences. The optimal simple rule will solve for the values of $F$ that minimize the loss function given by (5.16) subject to the economic system denoted by $(5.14)$

To find optimal simple rules, we first substitute $\hat{R}_{t}=-F X_{t}$ in $(5.14)^{78}$ and obtain

$$
A_{1}\left[\begin{array}{l}
X_{1, t+1} \\
E_{t} X_{2, t+1}
\end{array}\right]=\left(A_{2}-B F\right)\left[\begin{array}{l}
X_{1, t} \\
X_{2, t}
\end{array}\right]+\left[\begin{array}{l}
\varepsilon_{t+1} \\
0_{2}
\end{array}\right]
$$

Next, taking expectations conditional information set in period $t$, and letting $k_{t}=X_{1, t}$, and $h_{t}=X_{2, t}$, we then have the same type of problem as in (5.22), but with $G=A_{1}$, and $D=A_{2}-B F$. Assume that $F$ is appropriately set and the matrices $T_{u u}, S_{u u}$,

\footnotetext{
${ }^{17}$ Our presentation of the mechanics of simple optimal rules follows the work of Currie and Levine (1993).

${ }^{78}$ Equation (5.15) is modified to be $A_{1}\left[\begin{array}{l}X_{1, t+1} \\ E_{t} X_{2, t+1}\end{array}\right]=A_{2}\left[\begin{array}{l}X_{1, t} \\ X_{2, t}\end{array}\right]+B \hat{R}_{t}+\left[\begin{array}{l}\varepsilon_{t+1} \\ 0_{2}\end{array}\right]$.
} 
$S_{s s}$, and $Z_{k s}$ are all invertible, then the solution to (6.1) is analogous to (5.37) and (5.39), that is,

$$
\begin{aligned}
& {\left[X_{1, t+1}\right]=Z_{k s} S_{s s}^{-1} T_{s s} Z_{k s}^{-1}\left[X_{1, t}\right]+\left[\varepsilon_{t+1}\right]} \\
& {\left[X_{2, t}\right]=Z_{h s} Z_{k s}^{-1}\left[X_{1, t}\right]}
\end{aligned}
$$

and the welfare loss can be calculated by using (5.51) given $F$.

Note that thus far the optimal values of $F$ have not been calculated. To find the optimized values, we need to use a non-linear optimization algorithm from one of the advanced econometric or computational software programs such as Matlab or Dynare. In our case, the optimized reaction coefficients and associated welfare losses were obtained using Matlab. The sizes of shocks are enlarged compared to Chapter 4 . We set the standard deviation of the productivity shock at $\sigma_{A}=0.098$, and $\rho_{A}=0.9$. The aggregate demand (taste) shock is set as $\sigma_{1}=0.351$, and $\rho_{1}=0.9$. Similarly we set the parameters of our risk premium shock at $\sigma_{2}=0.05$, and $\rho_{2}=0.5$. Finally for the foreign output shock, we set $\sigma_{y^{*}}$ to be 0.07 , and $\rho_{y^{*}}$ to be 0.85 . Hence the welfare losses reported below are not comparable directly with the results reported in Chapter 4 .

\subsection{Inertial Simple Optimal Rules}

It is worth re-emphasizing that when an economy is hit by shocks, the rate of inflation and other economic variables deviate not only from their steady state value but from the perfectly flexible price level associated with temporary market clearing. It is in response to this observed departure from the flexible price value that the policy maker adjusts its policy instrument, the short-term interest rate, to bring the observed variables 
back into line with their designated targets. However, given the persistence in the underlying shocks to these variables and their interaction through time and incomplete information available to the policy maker, optimal policy often consists of a series of small adjustments in the same direction through time rather than a single immediate jump. This outcome, one that is typically observed in the data, is called monetary policy inertia (Woodford, 2002) and is most often mimicked in the policy rule through the use of a lagged interest rate. This introduces what is called interest-rate smoothing. Because we (and many others) find that smoothing rules perform better than rules without smoothing, we begin our comparative analysis of optimal simple rules in our small open economy by adopting interest-rate smoothing. At a later stage, the interest-rate smoothing term is dropped so that we can see how these optimal simple rules perform without the effect of smoothing. As we will see, in some cases the difference is significant, while in others the difference is only minor.

The general form of the optimal simple rule interest-rate smoothing when written in terms of domestic output prices can be expressed as ${ }^{79}$

$$
\hat{R}_{t}=\phi_{w} \hat{r}_{t}^{f}+\phi_{\pi} \hat{\Pi}_{H, t}+\phi_{x} x_{t}+\phi_{q} q_{g, t}+\phi_{\Psi} \hat{\Psi}_{t}+\phi_{r} \hat{R}_{t-1}
$$

Here we begin by setting $\phi_{r}$ equal to 0.8 . This is the value typically used in empirical studies (see Clarida et. al., 2000). The case $0<\phi_{r}<1$ generates interest rate inertia as the

\footnotetext{
${ }^{79}$ The general form differs slightly from that defined in Chapter 2 and used in Chapter 4 . The reason for this departure is because as Rotemberg and Woodford (1999) argue, that there is no any reason to restrict attention to the case $0 \leq \phi_{r}<1$, although only in that case the policy rule can be described as involving partial adjustment toward to a "target" interest rate dependent on current output and inflation. In section 6.3 we find that the optimized value of $\phi_{r}$ is greater than one. Finally if $\phi_{r}$ is a constant then we can rewrite the policy rule in the general form used previous chapters. In that case, the values of the optimized reaction coefficients, such as $\phi_{\pi}$, are found using the formula $\phi_{\pi}=\phi_{\pi}^{*} /\left(1-\phi_{r}\right)$, where $\phi_{\pi}^{*}$ is the statistic currently reported in the tables above. For example when $\phi_{r}=0.8, \phi_{\pi}$ for Rule 1 in Table 6.1. becomes 5.741 instead of 1.1482 . The welfare loss remains the same.
} 
policy maker adjusts only partially the short-term interest rate to the target rate defined in terms of the relevant sticky to flexible price gaps.

The optimized reaction coefficients and their associated welfare losses corresponding to the five simple monetary policy rules are reported in Tables 6.1 through $6.5,{ }^{80}$ where Rule 1 is our most comprehensive rule corresponding to the case where the monetary authority sets the short-term interest rate in response to changes in the inflation rate, the real GDP output gap, the real exchange rate gap, and the law of one price gap. This we call the comprehensive rule (C). Dropping the law of one price gap from Rule 1 gives us Rule 2, called the real exchange rate rule (ER). For comparative purposes, we replace the real exchange rate term in Rule 2 with the law of one price gap. This becomes Rule 3, the exchange rate pass-through monetary policy rule (PT). Rule 4 has no openness component, called more usually the Taylor rule (TR). Rule 5 further supposes that the monetary policy authority chooses to drop the real output gap to focus exclusively on inflation. This we call Inflation Targeting (IT).

The five tables from 6.1 through 6.5 then correspond to two sets of four different information scenarios. Tables 6.1 and 6.2 correspond to the case where the central bank can observe all contemporaneous variables and calculate the appropriate flexible price value. Table 6.3 corresponds to the information case where the central bank can observe contemporaneous variables but cannot calculate the appropriate flexible price values (and so must substitute the steady state value for the flexible price value). Table 6.4 corresponds to the case where the central bank can observe and calculate flexible price values only with a one period lag. Table 6.5 corresponds to the case where the model's variables can be observed only with a lag and where the flexible price values cannot be

\footnotetext{
${ }^{80}$ The alternative five simple monetary policy rules are defined more fully in Chapter 4.
} 
calculated at all. Hence the four different information scenarios correspond to optimal policy making with successively less current and/or relevant information. Finally, the two different sets of results correspond to the cases where the central bank targets inflation measured first in terms of domestic output prices (Tables 6.1, 6.3, 6.4 and 6.5) and then in terms of consumer prices (Table 6.2). ${ }^{81}$

If we begin by discussing the results presented in Table 6.1, where the central bank is assumed to be able to observe and calculate all necessary information (Case 1), the following general results can be noted.

First, the three optimal simple rules that involve the exchange rate in some form (Rules 1-3) perform best, each working at least as well as the Taylor Rule. Then of the three exchange rate rules, the comprehensive rule performs best, outperforming the Taylor rule. It should be noted that this outcome is now completely different from that arising in Chapter 4 where the Taylor rule always yielded the smallest welfare loss. In that chapter, however, the reaction coefficients were simply imposed on the analysis from the values typically employed in the literature rather than optimized. If one briefly scans the remaining four tables, it can be seen that the significance of including rather than excluding the exchange rate in the monetary policy rule continues to hold for all four information cases across all versions of the model.

\footnotetext{
${ }^{81}$ The simulation results in Cases 2,3, and 4 corresponding to the monetary authority measuring inflation rate using CPI prices are not presented, as did in Chapter 4, instead they are presented in Appendix A6.1.
} 


\section{Table 6.1}

\section{Case 1: Partially optimal simple rules and domestic output prices}

$$
\hat{R}_{t}=\phi_{w} \hat{r}_{H, t}^{f}+\phi_{\pi} \hat{\Pi}_{H, t}+\phi_{x} x_{t}+\phi_{q} q_{g, t}+\phi_{\Psi} \hat{\Psi}_{t}+0.8 \hat{R}_{t-1} .
$$

\begin{tabular}{l|l|l|l|l|l|l}
\hline \multirow{2}{*}{$\begin{array}{l}\text { Policy } \\
\text { Rule }\end{array}$} & \multicolumn{5}{|c|}{ Optimized reaction coefficients } & Welfare Loss \\
\cline { 2 - 6 } & $\phi_{w}$ & $\phi_{\pi}$ & $\phi_{x}$ & $\phi_{q}$ & $\phi_{\psi}$ & \\
\hline 1 (C) & 0.4439 & 1.1482 & -0.0311 & 0.1023 & -0.0542 & 0.1598 \\
\hline $2($ EX) & 0.4920 & 1.3348 & 0.0204 & 0.0046 & 0 & 0.1608 \\
\hline $\mathbf{3}$ (PT) & 0.4860 & 1.3553 & 0.0228 & 0 & -0.0037 & 0.1608 \\
\hline $\mathbf{4}$ (TR) & 0.4903 & 1.3460 & 0.0229 & 0 & 0 & 0.1608 \\
\hline 5 (IT) & 0.5276 & 1.4830 & 0 & 0 & 0 & 0.1610 \\
\hline
\end{tabular}

The second point is that the marginal welfare gain from incorporating some element of the exchange rate into the policy rule is small for the current case where information is "perfect". For example, the ratio between the smallest welfare loss (Rule 1) and the largest one (Rule 5) in Table 1 is 0.9925 . If we compare just those rules that involve the exchange rate and the Taylor rule (Rule 4), the ratio becomes even smaller. For example, the ratio of Rule 1 to Rule 4 is 0.9938 . Furthermore, if we compare Rules 2 and 3 to 4 , the ratio becomes 1 . In other words, the inclusion of some forms of the exchange rate into the policy rule (as in Rules 2 and 3) does not improve the welfare at all relative to the Taylor. This is consistent with the findings of Adolfson (2007) who argues on this basis that the improvement social welfare from incorporating an exchange rate term into an otherwise fully optimized policy rule will be practically zero, irrespective of the degree of the exchange rate pass-through. In response to this challenge, however, several points need to be emphasized. First, the conclusion that adding the exchange rate into the policy rule does not enhance the social welfare holds only under relatively strong 
conditions. For example, even in the current case some forms of exchange rate use have resulted in welfare improvement. More significantly, however, Adolfson takes neither the lack of information nor its uncertainty into account. As we will see below, when the central bank is given access to less and less accurate current information for deciding upon policy, the inclusion of the exchange rate in the policy rule is always the welfare enhancing. ${ }^{82}$ In some cases it will produce relatively large increases in welfare.

The third general finding that arises first in Table 6.1 is that the size of the optimized inflation coefficients, $\phi_{\pi}$, are much larger than the other reaction coefficients. This result is typical of both ours and others findings and not at all unexpected. That is, given the calibrated values of the loss function derived from the model's utility function, the parameter values used in calibrating lead the policy maker to place a much higher weight on inflation in comparison with the other terms in the loss function. For example, given our calibrated values (and the re-scaling of the model so that the weight placed on the inflation rate is 1 ), the corresponding weights put on the other loss function gaps are: 0.0025 for the output gap; 0.00022 for the real exchange rate gap; and 0.0885 for the interest rate gap. Therefore to minimize the welfare loss the central bank must react strongly to inflation. This asymmetric weighting is also found in Rotemberg and Woodford (1999), for example. They report optimal values for the reaction coefficients corresponding to a Taylor rule with interest-rate smoothing of $1.22,0.06$, and 1.28, respectively, while without the interest-rate smoothing term, the optimal values found are 2.88 and 0.02 , respectively. It can also be shown in our model, for example, that by

\footnotetext{
${ }^{82}$ We should add a note of caution in that the arbitrary specificity of the utility function chosen for the analysis means that the welfare implications are better interpreted as ordinal indicators rather than cardinal measures. That is, the analysis is a better indicator of policies that are better or worse rather than as an indicator of exactly how much better or worse each policy is.
} 
increasing the weights in the utility function given to the output gap, the real exchange rate gap, and the interest rate gap to $0.05,0.05$, and 0.5 , respectively, that the optimal parameter values on the output gap and the Wicksellian natural rate corresponding to the Taylor rule (Rule 4) are increased to 0.2943 and 0.7174 , respectively, while the reaction coefficient associated with the inflation rate decreases to 1.0291 .

The fourth new finding is that the signs of the optimized reaction coefficients are not always positive, except for inflation. This is also similar to findings reported in Rotemberg and Woodford (1999). While such findings seem counter-intuitive, the likely explanation for the occasional negative coefficient is that when the shocks to the economy result in a positive output gap and higher inflation, the optimal inflation reaction coefficient is so large that it produces an overshooting of the interest rate relative to the output gap, hence requiring a perverse response to the output gap to minimize the loss function. Some support for this interpretation is given when for these perverse cases we change the weighting of the loss function. For example, if we use the revised reweighting of the loss function reported immediately above, the optimized reaction coefficients corresponding to Rule 1 are found to result in the following rule

$$
\hat{R}_{t}=0.8 \hat{R}_{t-1}+0.81 \hat{r}_{H, t}+1.17 \hat{\Pi}_{H, t}+0.09 x_{t}+0.37 q_{g, t}-0.19 \hat{\Psi}_{t}
$$

where the sign of optimized coefficient on the output gap now is positive instead of negative. Note that the welfare losses reported in Table 6.1 are all global minima. Then if we require the $\phi_{x}$ in Rule 1 to be positive (as above), the equilibrium is still found to be determinate but will yield a larger welfare loss. In this case, the welfare loss becomes 0.1615 . 
Lastly, as one would expect, the welfare losses arising under the optimized reaction coefficients are much smaller than their counterpart when the reaction coefficients were simply assigned. The smallest welfare loss in Table 6.1 is 0.1598 , while using the assigned values of Chapter 4 the corresponding welfare loss is $0.2468{ }^{83}$ Using these welfare measures cardinally, the results imply that using the optimized values for reaction coefficients will result in the welfare losses being reduced by $54 \%$.

Table 6.1 reports the optimized reaction coefficients and the welfare losses associated with five different monetary policy rules when the monetary authority targets the inflation rate measured in terms of domestic output prices. Tables 6.2 reports the results for the same five money rules but requires the monetary authority to target inflation measured in terms of CPI prices. ${ }^{84}$ An examination of Table 6.2 shows that generally speaking, the findings observed in Table 6.1 are repeated. The important difference is that the welfare losses reported in Table 6.2 are larger than those arising in Table 6.1. This is consistent what we found in Chapter 4. Hence whether we use the reaction coefficients typically used in the literature or their optimized values for our model, the results imply that the monetary authority of a small open economy should focus on stabilizing inflation rates measured in domestic output prices rather than consumer prices. Note also that in this case the first three policy rules that involve the exchange rate (Rules 1-3) are strictly superior to the Taylor rule (Rule 4). The loss ratios between Rule 1 and Rule 4, Rule 2 and Rule 4, Rule 3 and Rule 4 are all less than one, $0.9846,0.9989$, and 0.9951 , respectively, whereas in Table 6.1 the welfare losses for Rules 2 and 3 were the same as the loss that arose under the Taylor rule.

\footnotetext{
${ }^{83}$ Note that the welfare loss $(0.2468)$ is calculated by using the enlarged sizes of shocks and Matlab. ${ }^{84}$ Once again the difference in the two measures of inflation is that consumer price inflation incorporates the domestic prices of imported goods.
} 


\section{Table 6.2}

\section{Case 1: Partially optimal simple rules and CPI prices}

$$
\hat{R}_{t}=\phi_{w} \hat{r}_{t}^{f}+\phi_{\pi} \hat{\Pi}_{t}+\phi_{x} x_{t}+\phi_{q} q_{g, t}+\phi_{\Psi} \hat{\Psi}_{t}+0.8 \hat{R}_{t-1} .
$$

\begin{tabular}{l|l|l|l|l|l|l}
\hline \multirow{2}{*}{$\begin{array}{l}\text { Policy } \\
\text { Rule }\end{array}$} & \multicolumn{5}{|c|}{ Optimized reaction coefficients } & Welfare Loss \\
\cline { 2 - 6 } & $\phi_{w}$ & $\phi_{\pi}$ & $\phi_{x}$ & $\phi_{q}$ & $\phi_{\psi}$ & \\
\hline 1 (C) & 0.3506 & 0.8356 & -0.0397 & 0.1161 & -0.0705 & 0.1791 \\
\hline 2 (EX) & 0.4466 & 1.2250 & 0.0314 & -0.0189 & 0 & 0.1817 \\
\hline 3 (PT) & 0.4171 & 1.1676 & 0.0205 & 0 & -0.0179 & 0.1810 \\
\hline 4 (TR) & 0.4701 & 1.2363 & 0.0199 & 0 & 0 & 0.1819 \\
\hline 5 (IT) & 0.5071 & 1.3578 & 0 & 0 & 0 & 0.1820 \\
\hline
\end{tabular}

The cases reported in Tables 6.1 and 6.2 assume that the policy maker possesses an information set that includes all relevant contemporaneous variables, that is, that the monetary authority can observe all contemporaneous market variables and has sufficient information to calculate all the flexible price values required to implement the money rule. In Case 2 we remove information from the monetary authority by assuming that the ability to observe contemporaneous market values is insufficient to determine the flexible price values required to determine the targeted gaps in the money rules. Hence Table 6.3 reports the results for the five monetary rules when the policy maker must replace the Wicksellian natural interest rate and the other unobserved flexible price variables in the money rules with their steady state values.

As one would expect, a comparison of the welfare losses in Table 6.3 with those reported for the same measure of inflation in Table 6.1 shows that the welfare losses in Table 6.3 are always bigger than those in Table 6.1. A similar result obtains when we compare Table A6.1 (see Appendix A6.1) to Table 6.2. This is consistent with what we 
saw in Chapter 4. That is, the less information that is available to the authority making the policy decision, the larger will be the resulting welfare loss (the smaller the welfare gain that can be made from pursuing monetary policy optimally).

But while the overall welfare losses are all larger, the same pattern of welfare losses continues to arise across the five different monetary rules. In particular, the three exchange rate rules now always dominate the Taylor and Inflation Targeting money rules. Within the set of different exchange rate rules, there is some variation. For example, in Table 6.3 Rules 1 and 3 generate equally good welfare outcomes. This should not be entirely surprising since in Case 2 , Rules 1 and 3 are almost identical ${ }^{85}$

\section{Table 6.3}

Case 2: Partially optimal simple rules and domestic output prices

$$
\hat{R}_{t}=\phi_{\pi} \hat{\Pi}_{H, t}+\phi_{x} \hat{Y}_{t}+\phi_{q} q_{t}+\phi_{\psi} \hat{\Psi}_{t}+0.8 \hat{R}_{t-1}
$$

\begin{tabular}{l|l|l|l|l|l}
\hline \multirow{2}{*}{ Policy Rule } & \multicolumn{4}{|c|}{ Optimized reaction coefficients } & \multirow{2}{*}{ Welfare Loss } \\
\cline { 2 - 5 } & $\phi_{\pi}$ & $\phi_{x}$ & $\phi_{q}$ & $\phi_{\psi}$ & \\
\hline $1(\mathrm{C})$ & 7.6458 & 0.1583 & -0.0125 & -0.0576 & 0.1888 \\
\hline $2(\mathbf{E X )}$ & 7.8389 & 0.2052 & -0.0811 & 0 & 0.1889 \\
\hline $3(\mathbf{P T})$ & 7.6229 & 0.1517 & 0 & -0.0624 & 0.1888 \\
\hline $4(\mathrm{TR})$ & 7.7623 & 0.1733 & 0 & 0 & 0.1890 \\
\hline $5($ IT) & 10.6935 & 0 & 0 & 0 & 0.1944 \\
\hline
\end{tabular}

Finally, we note that the results for the optimal monetary rules in Table 6.3 also generate reaction coefficient values for the exchange rate and the law of one price that are

\footnotetext{
${ }^{85}$ In Case 2 when the model is expressed in terms of domestic output prices, Rule 1 can be rewritten as $\hat{R}_{t}=\left[\phi_{\pi}+(1-\alpha) \phi_{\psi}\right] \hat{\Pi}_{H_{, t}}-(1-\alpha) \phi_{\psi} \hat{\Pi}_{F, t}+\phi_{x} \hat{Y}_{t}+\left(\phi_{q}+\phi_{\psi}\right) \hat{q}_{t}-\phi_{\psi} \hat{q}_{t-1}+\phi_{\psi} \hat{\Psi}_{t-1}+\phi_{r} \hat{R}_{t-1}$, and Rule 3 is $\hat{R}_{t}=\left[\phi_{\pi}+(1-\alpha) \phi_{\psi}\right] \hat{\Pi}_{H, t}-(1-\alpha) \phi_{\psi} \hat{\Pi}_{F, t}+\phi_{x} \hat{Y}_{t}+\phi_{\psi} \hat{q}_{t}-\phi_{\psi} \hat{q}_{t-1}+\phi_{\psi} \hat{\Psi}_{t-1}+\phi_{r} \hat{R}_{t-1}$.
} 
often negative. In part, it may be that because these negative coefficients arise in those cases where the inflation reaction coefficient is quite large that the interest rate is overshooting with respect to the exchange rate and so requires perverse exchange rate adjustments to preserve determinacy. It also suggest, however, that sign (as well as the size) of the reaction coefficients may be affected not only by the loss function chosen but also by the information set assumed available to the policy maker.

In Tables 6.4 and 6.5 we repeat the procedure followed in Tables 6.1 and 6.3 , except for the information that is assumed to be available to the policy maker. In Tables 6.1 to 6.3 , we assumed that the policy maker could access current period information on market variables, while in Tables 6.4 and 6.5 we assume that the monetary authority can observe or access market values only with a one period lag. Case 3 follows Case 1 in assuming that the monetary authority can use its market information to calculate the flexible price values (but with a one period lag), while Case 4 follows Case 2 in assuming that the monetary authority cannot calculate the flexible price values and must use steady states values.

Broadly speaking, the removal of contemporaneous information does not change the basic ranking of monetary policy rules observed in Tables $6.1-6.3$. That is, in all these Tables at least one of the three exchange rate rules dominates the Taylor and/or Inflation Targeting money rule. The results then reinforce the earlier suggestion that for a small open economy some incorporation of external constraints on the economy in the exchange rate rule will dominate money rules that focus only on the traditional "closed" economy features of the money rule. 
However, while the general pattern of results may remain the same, three observations are worth pointing out. First, as expected, the withdrawal of information from the monetary authority increases the overall welfare loss. Comparing directly comparable tables, such as Tables 6.4 and 6.1, we see that the minimum welfare losses in Tables 6.4 and 6.5 are all larger than found in Tables 6.1 through 6.3. Using our numbers to compare the outcomes under Case 3 to Case 1 as an example, the ability of a central bank to access current period information would result in the welfare improvement of $1.19 \%$ compared to the result obtained when continuing to use the same information but with a one period lag.

Second, it is interesting to note that the welfare losses in Case 3 are all significantly smaller than those found for Case 2 . Note, for example, that the welfare loss arising from the use of Rule 1 in Case 3 (see Table 6.4) is 0.1617 , while being 0.1888 in Case 2 (see Table 6.3). The former is only $85.65 \%$ of the latter while the same ratio for the Taylor rule is $85.82 \%$. The same result can be seen in relation to Tables A6.2 and A6.3 (see Appendix A6.1) in which the policy maker uses the CPI measure of inflation. This general finding is of particular interest because it suggests that the ability to use flexible price values in the money rule (even if that information is old) can dominate the ability to access more current information but use steady state values in the policy rule. Implicitly, even though Case 3 assumes that the central bank uses one period lagged information, the amount of information it has access to in using flexible price values 
incorporates more relevant information than does the use of current information in Case $2^{86}$ This outcome is also consistent with the findings from Chapter 4.

Finally, as would be expected, the welfare loss in Case 4 is the largest of all four cases considered here. This is the case where the monetary authority unambiguously has the least amount of information.

Table 6.4

Case 3: Partially optimal simple rules and domestic output prices

$$
\hat{R}_{t}=\phi_{w} \hat{r}_{H, t-1}^{f}+\phi_{\pi} \hat{\Pi}_{H, t-1}+\phi_{x} x_{t-1}+\phi_{q} q_{g, t-1}+\phi_{\Psi} \hat{\Psi}_{t-1}+0.8 \hat{R}_{t-1}
$$

\begin{tabular}{l|l|l|l|l|l|l}
\hline \multirow{2}{*}{$\begin{array}{l}\text { Policy } \\
\text { Rule }\end{array}$} & \multicolumn{5}{|c|}{ Optimized reaction coefficients } & \multirow{2}{*}{ Welfare Loss } \\
\cline { 2 - 6 } & $\phi_{w}$ & $\phi_{\pi}$ & $\phi_{x}$ & $\phi_{q}$ & $\phi_{\psi}$ & \\
\hline $1(\mathrm{C})$ & 0.5307 & 1.4317 & -0.0411 & 0.0925 & -0.0475 & 0.1617 \\
\hline $2(\mathrm{EX})$ & 0.5813 & 1.5925 & 0.0015 & 0.0079 & 0 & 0.1622 \\
\hline 3 (PT) & 0.5727 & 1.6103 & 0.0054 & 0 & -0.0020 & 0.1622 \\
\hline $4(\mathrm{TR})$ & 0.5763 & 1.6076 & 0.0055 & 0 & 0 & 0.1622 \\
\hline $5($ IT) & 0.5896 & 1.6525 & 0 & 0 & 0 & 0.1623 \\
\hline
\end{tabular}

Table 6.5

Case 4: Partially optimal simple rules and domestic output prices

$$
\hat{R}_{t}=\phi_{\pi} \hat{\Pi}_{H, t-1}+\phi_{x} \hat{Y}_{t-1}+\phi_{q} \hat{q}_{t-1}+\phi_{\Psi} \hat{\Psi}_{t-1}+0.8 \hat{R}_{t-1}
$$

\begin{tabular}{l|l|l|l|l|l}
\hline \multirow{2}{*}{ Policy Rule } & \multicolumn{4}{|c|}{ Optimized reaction coefficients } & \multirow{2}{*}{ Welfare Loss } \\
\cline { 2 - 5 } & $\phi_{\pi}$ & $\phi_{x}$ & $\phi_{q}$ & $\phi_{\psi}$ & \\
\hline $1($ C) & 3.5134 & 0.0635 & -0.0167 & -0.0834 & 0.1955 \\
\hline $2($ EX) & 3.5598 & 0.1331 & -0.1268 & 0 & 0.1966 \\
\hline $3($ PT) & 3.5061 & 0.0552 & 0 & -0.0899 & 0.1955 \\
\hline
\end{tabular}

${ }^{86}$ We will show below that when $\phi_{r}$ is optimized instead of simply setting it to be 0.8 here, the finding will be modified. 


\begin{tabular}{l|l|l|l|l|l}
\hline 4 (TR) & 3.5071 & 0.0879 & 0 & 0 & 0.1981 \\
\hline $5($ IT) & 4.2006 & 0 & 0 & 0 & 0.2030 \\
\hline
\end{tabular}

A summary of our findings thus far leads to the following tentative conclusions. First, in a small open economy where asset markets are not perfect and where the degree of exchange rate pass through is incomplete, the exchange rate is an important endogenous variable that the policy maker should incorporate into its policy rule. Doing so will lower welfare losses. Second, the conclusion that the exchange rate (or exchange rate pass through) should be part of the money rule could not be reached by using the traditional (but essentially arbitrary) reaction coefficients (as shown in Chapter 4). This reinforces the case for using optimal reaction coefficients in the analysis. Third, while it is not surprising that the better is the information used by the policy maker, the larger will be the welfare gain associated with using monetary policy optimally, what is interesting is that the better the information accessible to the policy maker, the less will be the marginal welfare gain from incorporating the exchange rate into the policy rule. Our analysis then suggests that the use of the exchange rate in the policy rule will be more valuable to those small open economy monetary authorities with less good information on the current state of their economy. Finally, when considering the choice of the price index to be used as the measure of inflation in the monetary rule, our analysis suggests that the central bank should measure inflation in terms of domestic output prices.

\subsection{Optimal Smoothing Rules}

Strictly speaking the "optimal simple rules" presented in Tables 6.1 through 6.5 are optimal only in a restricted sense since the reaction coefficient on the interest rate 
smoothing term was assigned as $\phi_{r}=0.8$. Our reason for beginning by imposing that restriction on the money rules is that most estimates of $\phi_{r}$ fall in a small range about 0.8 . For example, Clarida et al (2000) find that the estimate of $\phi_{r}$ is 0.68 for pre-Volcker period, and 0.79 for Volcker-Greenspan era. Across six different quarterly U.S. data samples (differing in their definition of output gap), Kozicki (1999) reports a range for $\phi_{r}$ from 0.75 to 0.82 , while across 16 different quarterly samples of U.S. data (differing in output gap, inflation, and sample period definition), Amato and Laubach (1999) report a range of $\phi_{r}$ from 0.78 to 0.92 . However, even though our chosen value falls in the middle of these findings, it is interesting to ask how the optimal simple policy rules respond to the lifting of that restriction.

To investigate the consequences of allowing the coefficient of interest rate smoothing term to be determined through the minimization process, we redo the analysis of Tables $6.1,6.3,6.4$, and 6.5 letting $\phi_{r}$ be a variable instead of imposing it as a constant. ${ }^{87}$ The results of this re-optimizing process are presented in Tables 6.6 through 6.9. Because the results of endogenizing interest rate smoothing are similar across the cases, we restrict specific comment to the observations that can be seen in Table 6.6 .

\footnotetext{
87 That is, to save on space we present only the results for the different information cases when the monetary authority responds to inflation rates measured in terms of domestic output prices. The corresponding cases where the monetary authority uses consumer prices are broadly similar.
} 


\section{Table 6.6}

\section{Case 1: Fully optimal inertial simple rules}

$$
\hat{R}_{t}=\phi_{w} \hat{r}_{H, t}^{f}+\phi_{\pi} \hat{\Pi}_{H, t}+\phi_{x} x_{t}+\phi_{q} q_{g, t}+\phi_{\Psi} \hat{\Psi}_{t}+\phi_{r} \hat{R}_{t-1} .
$$

\begin{tabular}{|c|c|c|c|c|c|c|c|}
\hline Policy & \multicolumn{6}{|c|}{ Optimized reaction coefficients } & \multirow{2}{*}{$\begin{array}{l}\text { Welfare } \\
\text { Loss }\end{array}$} \\
\hline Rule & $\phi_{w}$ & $\phi_{\pi}$ & $\phi_{x}$ & $\phi_{q}$ & $\phi_{\psi}$ & $\phi_{r}$ & \\
\hline $1(\mathrm{C})$ & $\begin{array}{l}-0.0570 \\
(0)\end{array}$ & $\begin{array}{l}2.1011 \\
(2.0456)\end{array}$ & $\begin{array}{l}0.0107 \\
(0.008)\end{array}$ & $\begin{array}{l}0.0036 \\
(0.0089)\end{array}$ & $\begin{array}{l}0.0302 \\
(0.0245)\end{array}$ & $\begin{array}{l}1.9290 \\
(1.8246)\end{array}$ & $\begin{array}{l}0.1586 \\
(0.1586)\end{array}$ \\
\hline $2(\mathbf{E X})$ & $\begin{array}{l}0.0725 \\
(0)\end{array}$ & \begin{tabular}{l|}
1.9345 \\
$(2.0073)$
\end{tabular} & $\begin{array}{l}-0.0077 \\
(-0.0092)\end{array}$ & $\begin{array}{l}0.0392 \\
(0.0412)\end{array}$ & 0 & $\begin{array}{l}1.6606 \\
(1.7857)\end{array}$ & $\begin{array}{l}0.1587 \\
(0.1587)\end{array}$ \\
\hline 3 (PT) & $\begin{array}{l}-0.0657 \\
(0)\end{array}$ & $\begin{array}{l}2.1143 \\
(2.0625)\end{array}$ & $\begin{array}{l}0.0125 \\
(0.0126)\end{array}$ & 0 & $\begin{array}{l}0.0324 \\
(0.0289)\end{array}$ & $\begin{array}{l}1.9464 \\
(1.8304)\end{array}$ & $\begin{array}{l}0.1586 \\
(0.1586)\end{array}$ \\
\hline 4 (TR) & $\begin{array}{l}0.0691 \\
(0)\end{array}$ & \begin{tabular}{l|}
2.0736 \\
$(2.1472)$
\end{tabular} & $\begin{array}{l}0.0115 \\
(0.011)\end{array}$ & 0 & 0 & $\begin{array}{l}1.6583 \\
(1.7767)\end{array}$ & $\begin{array}{l}0.1589 \\
(0.1589)\end{array}$ \\
\hline 5 (IT) & $\begin{array}{l}0.0609 \\
(0)\end{array}$ & $\begin{array}{l}2.1932 \\
(2.2534)\end{array}$ & 0 & 0 & 0 & $\begin{array}{l}1.7155 \\
(1.818)\end{array}$ & $\begin{array}{l}0.1589 \\
(0.1589)\end{array}$ \\
\hline
\end{tabular}

* Note that the values in parenthesis are the optimized reaction coefficients and welfare loss yielded by the same rule when $\phi_{w}$ is set equal to zero.

First and the most interesting observation is our finding that the optimized value of $\phi_{r}$ is completely different from the one reported in the literature and the value used in section 6.2. Here we find the optimized coefficient is greater than one rather than less than one. This implies that the monetary policy process is not stable for all given initial values of the interest rate. As Rotemberg and Woodford (1999) point out, however, the interesting feature is that the "explosive" monetary rule does not produce explosive 'equilibria'. Rather, the explosive nature of the rule implies that the interest rate set must jump to a value that preserves the expected equilibrium value of the interest rate. That is, intuitively, the prospect of an exponential increase in real rate leads to a substantial 
reduction in expected future aggregate demand through the aggregate demand function, which in turn induces firm to cut prices today. That decline in prices ensures that the interest rate need not rise but instead converge on its equilibrium value.

The second change is that the absolute values of the optimized coefficient on the Wicksellian natural rate $\phi_{w}$ become much smaller when the coefficients on the lagged interest rate $\phi_{r}$ are optimized. They were much larger, around 0.5 , when $\phi_{r}$ was set equal to 0.8 . This suggests that there might be some degree of substitutability in how the two forms of interest rate appear in the money rule-the optimized values of the coefficients on the Wicksellian natural rate versus the optimized value the lagged interest rate coefficient. To test whether there is a trade-off arising between the Wicksellian rate and the lagged interest rate in the money rule, we experiment with different values in terms of Rule 2. Table 6.6 currently shows that the optimized values of $\phi_{w}$ and $\phi_{r}$ are 0.0725 , and 1.6606 , respectively. Suppose now that that we simply decrease $\phi_{r}$ and set it at 1.2. Reoptimization over the other values in the rule results the optimized value of $\phi_{w}$ increasing to 0.284 . Next, Table 6.1 has shown that when $\phi_{r}$ was set at $0.8, \phi_{w}$ becomes 0.4920 , and when we decrease $\phi_{r}$ even further to $0.2, \phi_{w}$ now jumps to 1.572 . Finally, Table 6.10 shows that $\phi_{w}$ further jumps to 3.128 when $\phi_{r}$ is set to be zero. This exercise is consistent with the hypothesis that the lagged interest rate term performs some of the same functions as the Wicksellian natural rate in the money rule.

A third observation arising when Table 6.6 is examined in conjunction with Table 6.7 is that when the lagged interest rate coefficient is optimized, difference in policy performance for each of the different monetary policy rules in Cases 1 and 2 is negligible 
despite the information loss arising in the second case. There appear to be two important reasons for this particular finding. First to the extent that the optimized lagged interest rate substitutes for the Wicksellian rate, the elimination of the Wicksellian rate in Case 2 should have relatively little effect on the overall outcome. This prediction, arising from the observation in point two above, can be tested by imposing a zero coefficient on the Wicksellian rate in Table 6.6 and then re-optimizing. The results of doing this are reported as the second line (in parentheses) of each row in Table 6.6. As the table shows, the removal of the Wicksellian natural rate term does not change the size of the welfare loss for each money rule. The second difference that arises between Cases 1 and 2 is in the use of flexible price output and law of one price gap in Case 1 versus the use of the deviation from the steady state in Case 2 . Then because the weights in the loss function ascribed to these two dimensions are quite small, the overall change in the welfare loss from using steady state values versus flexible price values is negligible. This latter point can be confirmed by increasing the weights placed on the output gap and the law of one price gap in the loss function. Doing so does now produce a larger deadweight loss for each money rule.

Fourth, the incorporation of optimal interest rate smoothing reduces even further the marginal welfare differences arising across the five different monetary policy rules. What this means for our earlier policy conclusion is that while optimal policy should still include some form of exchange rate consideration, the marginal gain from doing so is now considerably smaller. For example, the ratio of the welfare losses yielded by Rule 1, the smallest welfare loss, compared to the Taylor rule (Rule 4) in Table 6.6 is 0.9981 ; compared to Rule 2 and Rule 4 is 0.9987 , and the ratio of Rule 3 to Rule 4 is exactly the 
same as the ratio of Rule 1 to Rule 4. This result may not be that surprising. Below we will show that when we compare non-inertia policy rules to rules with interest rate smoothing, the policy rules with inertia always perform better. However, the marginal welfare gain is largest when any form of interest rate smoothing is adopted. The marginal gain then becomes smaller as the policy maker moves from an arbitrary reaction coefficient to one that is optimize. This mirrors our finding that the gain in the performance of the money rule increases at a decreasing rate as the policy maker access more and more information relevant to their policy making decision.

Finally, as we would expect, the welfare losses arising when the optimized coefficient is used (in Tables 6.6 through 6.9) are all smaller than those found when the 'traditional sized' smoothing rule is adopted (in Tables 6.1 through 6.5). For example, when the welfare losses summarized in Table 6.6 are compared directly to those listed for the same information case in Table 6.1, we find welfare gains across the five rules ranging from $0.9 \%$ to $1.4 \%$.

The results for the remaining information cases are presented below for comparison. In general, they simply reinforce the points made above. However, there is one new finding of interest--the welfare losses in Case 2 (see Table 6.7) are now smaller (rather than larger, see Tables 6.3 and 6.4) than those arising in Case 3 (see Table 6.8). This implies that even though the monetary authority in Case 2 is constrained to use steady state values in its targeted gaps, the use of an optimized (rather than arbitrary) coefficient for the lagged interest rate more than offsets the ability to use lagged flexible price values in the money rule. This finding, however, is again somewhat conditional on the loss function used. It can be shown, for example, that by increasing the utility weights 
given to the output gap, the real exchange rate gap, and the interest rate gap to $0.05,0.05$, and 0.5 , respectively, as we did earlier, yield welfare losses for Case 2 that are larger than those in Case 3 and also larger than those generated in Case 1. The welfare losses in Rule 2 from using the new weights in the loss function in Cases 1 to 3 are 1.0388, 1.0714, and 1.0633 , respectively.

Table 6.7

Case 2: Fully optimal inertial simple rules and domestic output prices

$$
\hat{R}_{t}=\phi_{\pi} \hat{\Pi}_{H, t}+\phi_{x} \hat{Y}_{t}+\phi_{q} \hat{q}_{t}+\phi_{\Psi} \hat{\Psi}_{t}+\phi_{r} \hat{R}_{t-1} .
$$

\begin{tabular}{l|l|l|l|l|l|l}
\hline \multirow{2}{*}{$\begin{array}{l}\text { Policy } \\
\text { Rule }\end{array}$} & \multicolumn{5}{|c|}{ Optimized reaction coefficients } & Welfare \\
\cline { 2 - 6 } & $\phi_{\pi}$ & $\phi_{x}$ & $\phi_{q}$ & $\phi_{\psi}$ & $\phi_{r}$ & Loss \\
\hline 1 (C) & 2.0541 & -0.0015 & 0.0184 & 0.0257 & 1.8238 & 0.1586 \\
\hline 2 (EX) & 2.0807 & -0.0143 & 0.0385 & 0 & 1.7888 & 0.1587 \\
\hline 3 (PT) & 2.1000 & 0.0077 & 0 & 0.0327 & 1.8374 & 0.1586 \\
\hline 4 (TR) & 2.2281 & 0.0024 & 0 & 0 & 1.8026 & 0.1589 \\
\hline 5 (IT) & 2.2534 & 0 & 0 & 0 & 1.8180 & 0.1589 \\
\hline
\end{tabular}

Table 6.8

Case 3: Fully optimal inertial simple rules and domestic output prices

$$
\hat{R}_{t}=\phi_{w} \hat{r}_{H, t-1}^{f}+\phi_{\pi} \hat{\Pi}_{H, t-1}+\phi_{x} x_{t-1}+\phi_{q} q_{g, t-1}+\phi_{\Psi} \hat{\Psi}_{t-1}+\phi_{r} \hat{R}_{t-1} .
$$

\begin{tabular}{|c|c|c|c|c|c|c|c|}
\hline Policy & \multicolumn{6}{|c|}{ Optimized reaction coefficients } & \multirow{2}{*}{$\begin{array}{l}\text { Welfare } \\
\text { Loss }\end{array}$} \\
\hline Rule & $\phi_{w}$ & $\phi_{\pi}$ & $\phi_{x}$ & $\phi_{q}$ & $\phi_{\psi}$ & $\phi_{r}$ & \\
\hline $1(\mathrm{C})$ & 0.4367 & 1.5505 & -0.0296 & 0.0673 & -0.0297 & 1.0040 & 0.1616 \\
\hline $2(\mathbf{E X})$ & 0.3450 & 1.6785 & -0.0078 & 0.0240 & 0 & 1.2157 & 0.1617 \\
\hline 3 (PT) & 0.3295 & 1.7321 & 0.0043 & 0 & 0.0095 & 1.2500 & 0.1618 \\
\hline 4 (TR) & 0.3687 & 1.7285 & 0.0037 & 0 & 0 & 1.1589 & 0.1618 \\
\hline 5 (IT) & 0.3746 & 1.7613 & 0 & 0 & 0 & 1.8887 & 0.1618 \\
\hline
\end{tabular}




\section{Table 6.9}

Case 4: Fully optimal inertial simple rules and domestic output prices

$$
\hat{R}_{t}=\phi_{\pi} \hat{\Pi}_{H, t-1}+\phi_{x} \hat{Y}_{t-1}+\phi_{q} \hat{q}_{t-1}+\phi_{\Psi} \hat{\Psi}_{t-1}+\phi_{r} \hat{R}_{t-1}
$$

\begin{tabular}{l|l|l|l|l|l|l}
\hline \multirow{2}{*}{$\begin{array}{l}\text { Policy } \\
\text { Rule }\end{array}$} & \multicolumn{5}{|c|}{ Optimized reaction coefficients } & Welfare \\
\cline { 2 - 7 } & $\phi_{\pi}$ & $\phi_{x}$ & $\phi_{q}$ & $\phi_{\psi}$ & $\phi_{r}$ & Loss \\
\hline 1 (C) & 1.8047 & -0.0024 & 0.0158 & 0.0197 & 1.7923 & 0.1620 \\
\hline 2 (EX) & 1.8221 & -0.0122 & 0.0311 & 0 & 1.7573 & 0.1621 \\
\hline 3 (PT) & 1.8344 & 0.0054 & 0 & 0.0255 & 1.8006 & 0.1621 \\
\hline 4 (TR) & 1.9116 & 0.0014 & 0 & 0 & 1.7523 & 0.1623 \\
\hline $5($ IT) & 1.9247 & 0 & 0 & 0 & 1.7613 & 0.1623 \\
\hline
\end{tabular}

\subsection{Non-Inertial Optimal Simple Rules}

As mentioned above, central bank behavior is usually modeled as sluggish, exhibiting interest rate inertia. However, not everyone agrees. Rudebusch (2002), for example, argues that if financial markets can be assumed to process information efficiently, then evidence from the term structure implies that empirical policy rules displaying substantial partial adjustment will be misspecified. Hence if we take Rudebusch's position on this issue, it is of interest to know what the optimal simple monetary policy rule would be in the absence of interest rate smoothing. From a different perspective, Chapter 4 suggested that the addition of an interest-rate smoothing term into a simple monetary policy rules will deliver superior policy performance in some cases. However, in that chapter the reaction coefficients were simply taken from values used in the literature rather than optimized. Hence a natural question is whether the introduction of an interest-rate smoothing term into an otherwise simple optimal money rule will still produce higher levels of social welfare? To answer that question we need to find the non- 
inertial optimized simple rules and their associated welfare losses and then compare them to the simple optimal rules with inertia found in Sections 6.2 and 6.3 .

To that end, we redo the analysis of Tables 6.1 through Table 6.5 by dropping the interest-rate smoothing term from each of the monetary rules. The optimized reaction coefficients and welfare losses associated with each rule are reported in Tables 6.10 through 6.14. Broadly speaking, an examination of these results allows two important sets of conclusions to be illustrated. First, by looking at the size of the welfare losses, most of the findings with respect to the relative strength of the alternative simple monetary rules found in Tables 6.1 to 6.9 will continue to hold. Second, however, a number of new observations arise. Our analysis proceeds by discussing explicitly only those cases that use domestic output prices. ${ }^{88}$

\section{Table 6.10}

\section{Case 1: Non-inertial optimal simple rules and domestic prices}

$$
\hat{R}_{t}=\phi_{w} \hat{r}_{H, t}^{f}+\phi_{\pi} \hat{\Pi}_{H, t}+\phi_{x} x_{t}+\phi_{q} q_{g, t}+\phi_{\Psi} \hat{\Psi}_{t}
$$

\begin{tabular}{l|l|l|l|l|l|l}
\hline \multirow{2}{*}{$\begin{array}{l}\text { Policy } \\
\text { Rule }\end{array}$} & \multicolumn{5}{|c|}{ Optimized reaction coefficients } & \multirow{2}{*}{ Welfare Loss } \\
\cline { 2 - 6 } & $\phi_{w}$ & $\phi_{n}$ & $\phi_{x}$ & $\phi_{q}$ & $\phi_{\psi}$ & \\
\hline $\mathbf{1}(\mathbf{C})$ & 1.8590 & 5.0801 & -0.0730 & 0.2825 & -0.2127 & 0.1719 \\
\hline $2(\mathrm{EX})$ & 3.1280 & 11.2058 & 0.2115 & -0.1538 & 0 & 0.1724 \\
\hline $\mathbf{3}(\mathrm{PT})$ & 2.7212 & 9.4060 & 0.1144 & 0 & -0.1065 & 0.1722 \\
\hline 4 (TR) & 3.1698 & 10.8912 & 0.1169 & 0 & 0 & 0.1727 \\
\hline $5($ IT) & 3.2723 & 11.5622 & 0 & 0 & 0 & 0.1729 \\
\hline
\end{tabular}

${ }^{88}$ Table 6.11 and other three tables (Table 6A.4, 6A.5 and 6A.6 are presented in Appendix 6A.2) correspond to the four information cases when the policy maker uses CPI inflation and are included for comparison. Their findings are broadly similar. Note also that the welfare losses in these cases are typically larger than their domestic price counterparts. 


\section{Table 6.11}

\section{Case 1: Non-inertial optimal simple rules and CPI prices}

$$
\hat{R}_{t}=\phi_{w} \hat{r}_{t}^{f}+\phi_{\pi} \hat{\Pi}_{t}+\phi_{x} x_{t}+\phi_{q} q_{g, t}+\phi_{\Psi} \hat{\Psi}_{t}
$$

\begin{tabular}{l|l|l|l|l|l|l}
\hline \multirow{2}{*}{$\begin{array}{l}\text { Policy } \\
\text { Rule }\end{array}$} & \multicolumn{5}{|c|}{ Optimized reaction coefficients } & Welfare Loss \\
\cline { 2 - 6 } & $\phi_{w}$ & $\phi_{\pi}$ & $\phi_{x}$ & $\phi_{q}$ & $\phi_{\psi}$ & \\
\hline 1 (C) & 1.6624 & 4.7558 & -0.0142 & 0.4171 & -0.3772 & 0.1975 \\
\hline 2 (EX) & 2.7067 & 10.5384 & 0.5676 & -0.3610 & 0 & 0.1995 \\
\hline 3 (PT) & 2.2758 & 8.5680 & 0.3068 & 0 & -0.2551 & 0.1982 \\
\hline 4 (TR) & 3.6136 & 13.9177 & 0.4489 & 0 & 0 & 0.2007 \\
\hline 5 (IT) & 3.1090 & 12.5820 & 0 & 0 & 0 & 0.2038 \\
\hline
\end{tabular}

Consider first how Table 6.10 compares to either Table 6.1 or Table 6.6 (cases that are the same except for the removal of the lagged interest rate term from the policy rule). By examining the expected welfare losses under the different policy rules, we easily see that our key earlier observation continues to hold. That is, monetary policy rules that involve the exchange rate in some form yield smaller welfare losses than the rules that exclude such external considerations. In addition, of the three exchange rate rules, the comprehensive rule once again works best. This pattern of outcomes can be seen in all the tables, from 6.10 to 6.14 . It is also important to note that the marginal welfare gain from incorporating an exchange rate effect into the non-inertial optimal policy rule is non-negligible. Unlike the interest rate smoothing results, each of the three exchange rate cases dominates the Taylor and Inflation Targeting rules. This reinforces our general theme that a small open economy should incorporate some representation of the exchange rate into its money rule. 
Second, by comparing the welfare losses reported in Tables 6.10 to those in Table 6.1, we see that the welfare losses reported in Table 6.10 are much larger. For example, the smallest welfare loss yielded by Rule 1 in Table 6.10 is 0.1719 versus 0.1598 in Table 6.1. The former is 1.076 times the later. This finding also holds when we compare the welfare losses in Tables 6.11 to 6.2 , and in all other relevant table comparisons. Hence the addition of the interest-rate smoothing term to the optimal simple policy rule produces superior policy performance across all our four information cases and the two different inflation rate versions of the model. This contrasts with the earlier analysis of Chapter 4 , where the addition of interest-rate smoothing delivered better policy performances only in Cases 2 and 4 where the monetary authority was constrained to use steady state values. In Cases 1 and 3, where flexible price values could be used, interest rate smoothing made things worse. While our optimized coefficient cases always support the choice of an exchange rate effect in the money rule, the combination of these observations suggests the following generalization. If the policy maker has access to less information, in our case as the policy maker moves from Case 1 through Case 4, the efficiency case for augmenting any simple monetary policy rule with interest-rate smoothing is strengthened. That is, whether the reaction coefficients are optimized or not, the money rule will perform better with interest rate smoothing the less good is information held by the monetary authority. Conversely, as more relevant information is acquired by the policy maker (as we move towards Case 1), the incorporation interestrate smoothing into a simple policy rule produces less benefit and may in some circumstances actually reduce policy performance (see Chapter 4 , for example). 
This latter point becomes our third important finding. That is, the more accurate is the information that the policy maker can obtain, the less is the gain in policy efficiency from interest-rate smoothing. While this point may seem obvious, it is nice to have it confirmed formally within the model. For example, consider the situation in which the policy maker targets the domestic output price inflation. In this case, the welfare loss is 0.2376 under Rule 1 without interest-rate smoothing in Case 4 (see Table 6.14), while with partially interest rate smoothing it becomes 0.1955 (see Table 6.5). Hence adding interest-rate smoothing into the optimal simple rule increases (decreases) welfare (the welfare loss) by $21.5 \%$. On the other hand, when the monetary authority has its most relevant information (corresponding to Case 1), the addition of interest rate smoothing increases welfare only by $7.6 \%$ under Rule 1 . The corresponding reductions for the intermediate Cases 2 and 3 are $1.3 \%$ and $1.8 \%$, respectively. Note that Tables 6.1 through 6.5 the reaction coefficients are not fully optimized since the coefficient on the lagged interest rate is arbitrarily set equal to 0.8 . Using statistics reported in Table 6.6 through 6.9 in which the all reaction coefficients are optimized, the corresponding reductions under Rule 1 for four cases (Cases 1 through 4 ) are $8.4 \%, 20.6 \%, 1.9 \%$, and $46.7 \%$, respectively.

Fourth, in Table 6.1 we first noticed the result that the optimized reaction coefficients for inflation were always larger than the other optimized coefficients. This feature appears again but magnified much further once interest rate smoothing has been removed. For example, in Table 6.1, the values of $\phi_{\pi}$ range from 1.15 to 1.48 , while in Table 6.10, the smallest value of $\phi_{\pi}$ is 5.08 and the largest 11.56. A similar result can be observed in relation to the coefficients on the Wicksellian natural rate, $\phi_{w}$. They are all 
now greater than one and much larger than those observed in Table 6.1 and the other tables. The intuition for this finding is similar to that given earlier when the fully optimized coefficient on the lagged interest rate, $\phi_{r}$, exceeded one. When the inflation rate rises due to a shock, for example, the central bank needs to increase its short-term interest rate significantly to bring down the expected future aggregate demand sufficiently to leads firms today to start cutting prices.

Turning next to Case 2 in Table 6.12, we see that the welfare losses associated with each rule are much larger than those reported in Table 6.10. This is consistent with the finding when the policy rule had interest rate smoothing. However, since there is less information in Case 2 than in Case 1, the central bank that hopes to reduce welfare losses needs to react strongly to the current deviation in inflation since the marginal gain from reacting is becoming smaller and smaller. For example, to bring down the welfare loss associated with the Taylor rule to $0.1942, \phi_{\pi}$ needs to jump 50 , and to bring down to $0.1921, \phi_{\pi}$ must jump to 200 . Finally, to reach the minimized welfare loss $(0.1914), \phi_{\pi}$ needs to dramatically jump to $1.2544 \mathrm{e}+06$.

When comparing Case 2 with Case 1, the key missing piece of information is the Wicksellian natural rate. Suppose then that we introduce it back into Case 2 and set $\phi_{w}=1.1$. Given the other conditions, the welfare loss yielded by the Taylor rule decrease from 0.1913 to 0.1859 and the optimized reaction coefficients on inflation and the deviation of output become 3.2116 and 0.0427 , respectively. In contrast, if we just get the output gap back, it will deteriorate the social welfare $(0.1944)$, and also requires the central bank in response to the variation in inflation very strongly. In the previous section we have seen that the effect of the missing of the Wicksellian natural rate on the social 
welfare can be offset completely by augmenting the lagged interest rate into the policy rule.

\section{Table 6.12}

Case 2: Non-inertial optimal simple rules and domestic output prices

$$
\hat{R}_{t}=\phi_{\pi} \hat{\Pi}_{H, t}+\phi_{x} \hat{Y}_{t}+\phi_{q} \hat{q}_{t}+\phi_{\Psi} \hat{\Psi}_{t}
$$

\begin{tabular}{l|l|l|l|l|l}
\hline \multirow{2}{*}{ Policy Rule } & \multicolumn{4}{|c|}{ Optimized reaction coefficients } & \multirow{2}{*}{ Welfare Loss ${ }^{* *}$} \\
\cline { 2 - 5 } & $\phi_{\pi}{ }^{*}$ & $\phi_{x}$ & $\phi_{q}$ & $\phi_{\psi}$ & \\
\hline 1 (C) & 10 & 0.2938 & 0.0403 & 0.0458 & $0.2103(0.1913)$ \\
\hline 2 (EX) & 10 & 0.2609 & 0.0926 & 0 & $0.2103(0.1913)$ \\
\hline 3 (PT) & 10 & 0.3136 & 0 & 0.0617 & $0.2103(0.1913)$ \\
\hline 4 (TR) & 10 & 0.2954 & 0 & 0 & $0.2104(0.1914)$ \\
\hline 5 (IT) & 10 & 0 & 0 & 0 & $0.2179(0.1944)$ \\
\hline
\end{tabular}

* Here $\phi_{\pi}=10$ is set arbitrarily rather than optimized. The optimized reaction coefficients and the associated welfare losses are obtained given this constraint imposed.

** The numbers in parentheses denote the minimized welfare losses when $\phi_{\pi}$ is optimized as well.

Finally, let us consider Tables 6.13 and 6.14 . Here the policy maker responds to information with a one period lag. Compared to Cases 1 and 2, Cases 3 and 4 present the policy maker with less information on which to decide the short-term interest rate. On this basis, Case 4 is expected to generate larger welfare losses than those in Case 2. This is confirmed by comparing the welfare losses reported in Table 6.14 to those reported in Table 6.12. Similarly, we would expect the welfare losses in Case 3 to be larger than those in Case 1. This was the result when interest rate smoothing was part of the policy rule (see the preceding section). However, in the current case, the outcome is completely opposite. Now the smallest welfare loss, 0.1646 , arises in Case 3 (see the first row of Table 6.13), whereas its corresponding value in Case 1 is 0.1719 (see the first row of 
Table 6.10). On the surface, this result is counterintuitive. However, given the importance of the lagged interest rate in the policy rule found earlier, finding that the lagged Wicksellian interest rate reduces welfare losses when there is no interest rate smoothing may not be too surprising. That is, this finding may simply mean that when the policy maker that doesn't use a lagged interest rate in the policy rule, the role played by the lagged interest rate in the money rule may be successfully substituted for by the adoption of the lagged Wicksellian natural rate $\left(\hat{r}_{H, t-1}^{f}\right)$ a function not picked up then the current period rate $\left(\hat{r}_{H, t}^{f}\right)$ is used in isolation.

To test this hypothesis we first use $\hat{r}_{H, t-1}^{f}$ to replace $\hat{r}_{H, t}^{f}$ in the policy rule without the interest-rate smoothing (Case 1) and see whether the welfare loss decreases or increases. Here a decrease in the size of the welfare loss would be consistent with our hypothesis, otherwise the hypothesis is contradicted. Secondly we substitute $\hat{r}_{H, t}^{f}$ for $\hat{r}_{H, t-1}^{f}$ in the Case 3 policy rule without the interest-rate smoothing. In this case, an increase in the size of the welfare loss is consistent with this hypothesis. The findings for both experiments are consistent with our hypothesis. Using $\hat{r}_{H, t-1}^{f}$ to replace $\hat{r}_{H, t}^{f}$ in the comprehensive rule and the Taylor rule without interest-rate smoothing in Case 1, the welfare losses are decreased from 0.1719 and 0.1727 to 0.1552 and 0.1557 , respectively. Conversely, when we substitute $\hat{r}_{H, t}^{f}$ for $\hat{r}_{H, t-1}^{f}$ in the comprehensive rule and the Taylor rule without the interest-rate smoothing in Case 3, the welfare losses rise from 0.1646 and 0.1676 to 0.1801 and 0.1857 , respectively. Finally, note that if our hypothesis holds and the Wicksellian gap is a superior target to the steady state gap, then not only should the lagged Wicksellian natural rate be able to replace the lagged interest rate, but it also 
should work better than the lagged interest rate in the policy rule. We test this latter hypothesis by adding $\hat{r}_{H, t-1}^{f}$ into the comprehensive rule without interest rate smoothing in Case 1 and generate a welfare loss of 0.1551 . However the welfare loss yielded by the comprehensive rule with inertia in Case 1 was 0.1586 (see the first row of Table 6.6).

This implies that using $\hat{r}_{H, t-1}^{f}$ to replace $\hat{R}_{t-1}$ has increased social welfare by $2.2 \%$.

\section{Table 6.13}

Case 3: Non-inertial optimal simple rules and domestic output prices

$$
\hat{R}_{t}=\phi_{w} \hat{r}_{H, t-1}^{f}+\phi_{\pi} \hat{\Pi}_{H, t-1}+\phi_{x} x_{t-1}+\phi_{q} q_{g, t-1}+\phi_{\Psi} \hat{\Psi}_{t-1}
$$

\begin{tabular}{l|l|l|l|l|l|l}
\hline \multirow{2}{*}{$\begin{array}{l}\text { Policy } \\
\text { Rule }\end{array}$} & \multicolumn{5}{|c|}{ Optimized reaction coefficients } & \multirow{2}{*}{ Welfare Loss } \\
\cline { 2 - 6 } & $\phi_{w}$ & $\phi_{\pi}$ & $\phi_{x}$ & $\phi_{q}$ & $\phi_{\psi}$ & \\
\hline $1(\mathbf{C})$ & 1.0936 & 1.5615 & -0.1381 & 0.2588 & -0.1483 & 0.1646 \\
\hline $2(\mathbf{E X )}$ & 1.4923 & 2.9743 & -0.0117 & -0.0196 & 0 & 0.1675 \\
\hline $3 \mathbf{( P T})$ & 1.4481 & 2.8938 & -0.0206 & 0 & -0.0211 & 0.1671 \\
\hline 4 (TR) & 1.5208 & 3.0011 & -0.0245 & 0 & 0 & 0.1676 \\
\hline 5 (IT) & 1.4931 & 2.8999 & 0 & 0 & 0 & 0.1676 \\
\hline
\end{tabular}

Table 6.14

Case 4: Non-inertial optimal simple rules and domestic output prices

$$
\hat{R}_{t}=\phi_{\pi} \hat{\Pi}_{H, t-1}+\phi_{x} \hat{Y}_{t-1}+\phi_{q} \hat{q}_{t-1}+\phi_{\Psi} \hat{\Psi}_{t-1}
$$

\begin{tabular}{l|l|l|l|l|l}
\hline \multirow{2}{*}{ Policy Rule } & \multicolumn{3}{|c|}{ Optimized reaction coefficients } & \multirow{2}{*}{ Welfare Loss } \\
\cline { 2 - 5 } & $\phi_{\pi}$ & $\phi_{x}$ & $\phi_{q}$ & $\phi_{\psi}$ & \\
\hline 1 (C) & 10.2087 & 0.1488 & -0.2375 & -0.3889 & 0.2376 \\
\hline 2 (EX) & 9.2452 & 0.4359 & -0.7003 & 0 & 0.2409 \\
\hline 3 (PT) & 10.0351 & 0.0318 & 0 & -0.4801 & 0.2380 \\
\hline 4 (TR) & 7.1149 & 0.1618 & 0 & 0 & 0.2495 \\
\hline 5 (IT) & 7.9879 & 0 & 0 & 0 & 0.2537 \\
\hline
\end{tabular}




\subsection{Robustness Analysis}

In Section 6.2 we highlighted two key findings of our analysis: first, the more relevant was the information incorporated in the policy decision, the larger was the welfare gain associated with using monetary policy optimally; and second, that in our small open economy, the exchange rate was an important endogenous variable whose incorporation into the policy rule improved the economy's performance. These outcomes were also found in Sections 6.3 and 6.4. In this section we examine the robustness of these findings as a basis for conclusions respecting the policy performance in small open economies such as ours.

One feature of our findings that could be seen as undermining confidence in the robustness of our exchange rate inclusion conclusion is the tendency, first observable in Table 6.1, for the welfare loss to decrease as additional variables are incorporated into the policy rule. This feature is observed in the other tables as well. This suggests the following counter hypothesis. Perhaps the reason that the comprehensive rule (Rule 1) results in a smallest welfare loss is simply because adding more variables will always improve the performance of a rule rather than because the exchange rate is itself an important targeting variable for the policy rule. ${ }^{89}$ Such a possibility suggests the following test: if the "more variables better outcome" hypothesis is true, then the addition of one or more 'significant' variables into the policy rule should yield an even smaller welfare loss.

\footnotetext{
${ }^{89}$ Adolfson (2007) raises a similar point. He points out that if the policy rule is excessively simple (i.e., is suboptimal) then the inclusion of any additional state variable is likely to yield an improvement in the rule. However, optimization of the reaction coefficients reduces the sub-optimality of the simple rule, which partly mitigates such a problem.
} 
To test this hypothesis, we first add "consumption" to Rule 1 in Case 1 when the monetary authority uses domestic output prices to target the inflation rate with the partial interest-rate smoothing (Table 6.1, row 1). In that case the welfare loss associated with the revised optimal policy rule was 0.1598 . Second if we instead add "labor" to Rule 1 , the welfare loss is also found to be 0.1598 . In both cases the welfare result is exactly the same as the welfare loss arising in their absence. Hence the simple inclusion of additional variables in the policy rule does not bring down the welfare loss further as would be expected. Similar tests were conducted for the cases where the monetary authority uses CPI prices to target the inflation rate. This set of findings gives us greater confidence that the reason that the comprehensive rule yields better policy performance is that the incorporation of the exchange rate and the law of one price gap into the policy rule incorporates more relevant information.

To further test this hypothesis, we re-optimize after taking the real exchange rate and the law of one price gap out of the policy rule and substituting "consumption" or "labor supply" in their place. Should these substitutions improve the performance of policy, our information hypothesis would be contradicted. We begin by replacing the real exchange rate in Case 1 Rule 1 with "consumption" in the policy rule and then doing the same for the law of one price gap. Our simulation results show that using "consumption" to replace either the real exchange rate or the law of price gap yields a larger welfare loss. Their respective values found were to be 0.1603 and 0.1603 , larger than the 0.1598 the welfare loss for the comprehensive Rule 1 reported in Table 6.1. Next, we use "labor" to replace the real exchange rate and the law of one price gap in Rule 1 and obtain the similar results. Here the welfare losses associated with each test are 
0.1608 and 0.1608 , respectively; both larger than the original 0.1598 loss arising under Rule 1 in Case 1. Finally, using both "consumption" and "labor" to replace the real exchange rate and the law of one price gap in Rule 1 improves performance (on their separate use) but also yields a larger over welfare loss, 0.1601 , compared to the comprehensive rule. Lastly, the exact same tests were repeated for the cases where the policy maker targets the inflation rate in terms of CPI prices with similar outcomes. ${ }^{90}$ Together these findings are strongly consistent with our interpretation of the information content of the openness variables - the real exchange and the law of one price gap-for the effectiveness of the money rule. As such, these findings increase our confidence in this interpretation of our results.

In our small economy model external considerations influence domestic outcomes in two ways: first through the assumption of incomplete capital markets and second through the law of one price gap. While the former forms part of the structure of the model, it has no one simple parameter by which the intensity of incompleteness can be adjusted. In this sense, the latter external complication is more susceptible to manipulation. In particular, the law of one price gap affects welfare via incomplete exchange rate pass-through, which in turn arises from the assumption that the importers face quadratic adjustment costs in setting prices in the domestic currency. In terms of the specification adopted, the parameter $\omega_{M}$ scales the magnitude of import price adjustment costs such that the degree of exchange rate pass-through increases as $\omega_{M}$ decreases. When $\omega_{M}$ is set equal to zero, the degree of exchange rate pass-through is 100 percent,

\footnotetext{
${ }^{90}$ Similar results were obtained when the coefficient on the lagged interest rate was optimized instead of when, as above, the coefficient was assigned the value of 0.8 . Finally using the programs of Dynare resulted in the same set of findings as when the programs were written in Matlab.
} 
which implies that the law of one price gap will be zero. In this case, adding to the policy rule the law of one price gap would not be expected to enhance the social welfare. Similarly since the size of the distortion in the real exchange rate is positively related to the law of one price gap, the marginal welfare gain from incorporating the real exchange rate into the policy rule should also fall as $\omega_{M}$ decreases, but because the real exchange rate is just partially related to the law of one price gap, therefore the marginal welfare gain from incorporating the real exchange rate will be expected to be less significant than those from Rules 1 and 3 as $\omega_{M}$ varies.

To test this implication of the analysis, we vary the parameter $\omega_{M}$ between 400 , which represents a low degree of exchange rate pass-through, and 1, representing a high degree of the exchange rate pass-through. ${ }^{91}$ The welfare losses $W L$ associated with the corresponding optimized simple rules and the relative welfare losses are presented in Tables 6.15 and 6.16. Table 6.15 shows that in relative terms the marginal social welfare gain from incorporating the exchange rate and/or the law of one price gap into the Taylor Rule is large when the degree of the exchange rate pass-through is low, while the gain is small under a high degree of the exchange rate pass-through. This is for the case where the policy maker responds to inflation measured in CPI prices. The same observation, however, does not appear as easily in Table 6.16, where the policymaker is assumed to react to the domestic output prices. We note that import prices are not in the measure of inflation targeted in this case and, in addition, conjecture that the interaction between incomplete pass-through rate and incomplete asset markets may play some role in this difference.

\footnotetext{
${ }^{91}$ In terms of Adolfson (2007) estimation, the degree of the exchange rate pass-through is about $10 \%$ when $\omega_{M}=400$, and $70 \%$ when $\omega_{M}=1$.
} 
Table 6.15

Case 1: The degree of exchange rate pass-through and CPI prices

\begin{tabular}{l|l|l|l|l|l|l|l}
\hline & \multicolumn{3}{|l|}{ Rule 1 } & \multicolumn{3}{l|}{ Rule 2 } & \multicolumn{2}{l}{ Rule 3 } & Rule 4 \\
\cline { 2 - 7 } & $W L_{1}$ & $W L_{1} / W L_{4}$ & $W L_{2}$ & $W L_{2} / W L_{4}$ & $W L_{3}$ & $W L_{3} / W L_{4}$ & $W L_{4}$ \\
\hline$\omega_{M}=400$ & 0.1975 & 0.9841 & 0.1995 & 0.9940 & 0.1982 & 0.9875 & 0.2007 \\
\hline$\omega_{M}=100$ & 0.2038 & 0.9869 & 0.2062 & 0.9985 & 0.2044 & 0.9898 & 0.2065 \\
\hline$\omega_{M}=50$ & 0.2059 & 0.9932 & 0.2072 & 0.9995 & 02061 & 0.9942 & 0.2073 \\
\hline$\omega_{M}=25$ & 0.2066 & 0.9995 & 0.2067 & 1 & 0.2066 & 0.9990 & 0.2067 \\
\hline$\omega_{M}=10$ & 0.2037 & 0.9985 & 0.2040 & 1 & 0.2038 & 0.9990 & 0.2040 \\
\hline$\omega_{M}=1$ & 0.1968 & 1 & 0.1968 & 1 & 0.1968 & 1 & 0.1968 \\
\hline
\end{tabular}

Table 6.16

Case 1: The degree of exchange rate pass-through and domestic output prices

\begin{tabular}{l|l|l|l|l|l|l|l}
\hline & \multicolumn{3}{|l|}{ Rule 1 } & \multicolumn{3}{l|}{ Rule 2 } & \multicolumn{2}{l|}{ Rule 3 } & Rule 4 \\
\cline { 2 - 8 } & $W L_{1}$ & $W L_{1} / W L_{4}$ & $W L_{2}$ & $W L_{2} / W L_{4}$ & $W L_{3}$ & $W L_{3} / W L_{4}$ & $W L_{4}$ \\
\hline$\omega_{M}=400$ & 0.1719 & 0.9954 & 0.1724 & 0.9983 & 0.1722 & 0.9971 & 0.1727 \\
\hline$\omega_{M}=50$ & 0.1727 & 0.9971 & 0.1732 & 1 & 0.1728 & 0.9977 & 0.1732 \\
\hline$\omega_{M}=25$ & 0.1732 & 0.9994 & 0.1733 & 1 & 0.1732 & 0.9994 & 0.1733 \\
\hline$\omega_{M}=10$ & 0.1731 & 0.9988 & 0.1733 & 1 & 0.1731 & 0.9988 & 0.1733 \\
\hline$\omega_{M}=1$ & 0.1709 & 0.9884 & 0.1729 & 1 & 0.1709 & 0.9884 & 0.1729 \\
\hline
\end{tabular}

As mentioned above, there is no one parameter by which we can vary the incompleteness of capital markets. However, the risk premium that the representative household must pay to borrow on world markets due to incomplete capital markets is variable and can be affected by the size of the shock to the risk premium, its net foreign 
asset position at the time of the shock, and the constant, $b$, which governs the size of the risk premium. ${ }^{92}$ Suppose then that while other conditions remain fixed, we let the size of the shock to the risk premium increase. Then since the larger the shock to the risk premium, the greater will be the effect of incomplete capital markets on the model leading to the larger the size of the welfare loss associated with that distortion. It then follows that the marginal gain from incorporating one of the external gaps into the money rule should increase as the size of shock to the risk premium increases. ${ }^{93}$

To test this implication, we redo Tables 6.15 and 6.16 for different sized shocks to the risk premium. The welfare losses $W L$ associated with the corresponding optimized simple rules and the relative welfare losses are presented in Tables 6.17 and 6.18 . Scanning Table 6.17 we see that the marginal welfare gains from adding one of the external gaps to the policy rules become more significant as the size of shock to the risk premium increases. For example, in Case 1 when $\varepsilon_{2 t}=0.05^{2}$, the relative gain associated with using the exchange rate in Rule 2 compared to the Taylor rule is $1 / 0.994=1.006$, while it becomes $1 / 0.8131=1.23$ when the size of the shock rises to $\varepsilon_{2 t}=1^{2}$. Similar observations can be made with respect to Table 6.18 .

\footnotetext{
${ }^{92}$ The risk premium is defined in Chapter 3 as $\exp \left(-b N F A_{t}+\ln \chi_{2 t}\right)$. In this sense, capital markets are complete when the risk premium is one.

${ }^{93}$ Cecchetti et al. (2000) show that financial disturbances may cause the exchange rate to have destabilizing effects that should be mitigated by monetary policy, while Adolfson (2007) does not support it.
} 
Table 6.17

Case 1: The size of the risk premium shock and CPI prices

\begin{tabular}{|c|c|c|c|c|c|c|c|}
\hline \multirow{2}{*}{$\begin{array}{l}\text { Risk } \\
\text { premium } \\
\text { shock }\end{array}$} & \multicolumn{2}{|l|}{ Rule 1} & \multicolumn{2}{|l|}{ Rule 2} & \multicolumn{2}{|l|}{ Rule 3} & \multirow{2}{*}{$\begin{array}{l}\text { Rule } 4 \\
W L_{4}\end{array}$} \\
\hline & $W L_{1}$ & $W L_{1} / W L_{4}$ & $W L_{2}$ & $W L_{2} / W L_{4}$ & $W L_{3}$ & $W L_{3} / W L_{4}$ & \\
\hline$\varepsilon_{2 t}=0.05^{2}$ & 0.1975 & 0.9841 & 0.1955 & 0.9940 & 0.1982 & 0.9875 & 0.2007 \\
\hline$\varepsilon_{2 t}=0.1^{2}$ & 0.2128 & 0.9668 & 02158 & 0.9805 & 0.2137 & 0.9709 & 0.2201 \\
\hline$\varepsilon_{2 t}=0.5^{2}$ & 0.7030 & 0.8346 & 0.7103 & 0.8524 & 0.7054 & 0.8465 & 0.8333 \\
\hline$\varepsilon_{2 t}=1^{2}$ & 2.1933 & 0.8041 & 2.2179 & 0.8131 & 2.2566 & 0.8273 & 2.7277 \\
\hline
\end{tabular}

Table 6.18

Case 1: The size of the risk premium shock and domestic output prices

\begin{tabular}{|c|c|c|c|c|c|c|c|}
\hline Risk & Rule 1 & & Rule 2 & & Rule 3 & & Rule 4 \\
\hline $\begin{array}{l}\text { premium } \\
\text { shock }\end{array}$ & $W L_{1}$ & $W L_{1} / W L_{4}$ & $W L_{2}$ & $W L_{2} / W L_{4}$ & $W L_{3}$ & $W L_{3} / W L_{4}$ & $W L_{4}$ \\
\hline$\varepsilon_{2 t}=0.05^{2}$ & 0.1719 & 0.9954 & 0.1724 & 0.9983 & 0.1722 & 0.9971 & 0.1727 \\
\hline$\varepsilon_{2 t}=0.1^{2}$ & 0.1833 & 0.9897 & 0.1840 & 0.9935 & 0.1836 & 0.9914 & 0.1852 \\
\hline$\varepsilon_{2 t}=0.5^{2}$ & 0.5381 & 0.9236 & 0,5656 & 0.9708 & 0.5447 & 0.9349 & 0.5826 \\
\hline$\varepsilon_{2 t}=1^{2}$ & 1.6441 & 0.9047 & 1.6705 & 0.9192 & 1.6702 & 0.9191 & 1.8173 \\
\hline
\end{tabular}

In Sections 6.2 and 6.4 we have shown that adding interest-rate smoothing term into optimal simple rules, broadly speaking, gives rise to better policy performance. One key question raised by this finding is why do simple feedback policy rules produce better policy performance with a lagged interest rate than without? Goodfriend (1991) argues that output and prices do not respond to daily fluctuations in short run interest rates, but rather to variations in the long-term interest rate. Hence to better achieve its stabilization 
goals, the central bank should try to affect these longer term rates. However, because long rates are determined by the market's expectation of future short run rates, an effective response to inflationary pressures requires the central bank to communicate a credible commitment to a changed future path of short rates. One straightforward way to do this, he argues, is for the central bank to establish a reputation for maintaining interest rates at a higher level for some period of time once they are raised, that is, follow an initial interest-rate change with further changes in the same direction. Such a policy, if understood by the private sector, offers the prospect of a more significant central bank policy effect upon aggregate demand without generating excessively volatile short-term interest rates.

Goodfriend's argument here is based on two key factors: forward-looking agents in an economic system and a central bank's ability to use commitment to an optimal policy rule to produce a better policy outcome than can be attained through optimization under discretion. However, while these two factors provide important reasons for expecting the success achieved by interest rate smoothing in our model, they may not capture all the reasons for that success. Here we explore two additional hypotheses for why in this model the addition of interest-rate smoothing to a simple optimal rule may deliver superior policy performance.

First of all it is often argued that the addition of the lagged interest rates may increase the relevant information available to the policy maker. To see whether this effect is present in our model, we calculate the relative welfare loss between each corresponding optimal simple policy rule with interest-rate smoothing and the policy rule without smoothing by using data from Table 6.6-6.9 and 6.10,6.12,6.13, and 6.14. The 
result is presented in Table 6.19, where $W L_{S}$ is used to denote the welfare loss associated with correspond optimal simple policy rule with interest-rate smoothing, and $W L_{N}$, the welfare loss without smoothing. Examining Table 6.19 we see that the improvement in performance of interest rate smoothing becomes larger in cases with less information in the model. As a general rule, the exception is Case 3. However, as was discussed earlier in section 6.4, the reason that the marginal welfare gain in Case 3 is smaller than that arising in Cases 1 and 2 is that in Case 3 at least part of the role played by the lagged interest rate $\hat{R}_{t-1}$ in money rule is replaced by the presence of the lagged Wicksellian natural rate, $\hat{r}_{H, t-1}^{f}$. Therefore it is because of the special appearance of the 'lagged' Wicksellian interest rate that the marginal welfare gain from adding the interest-rate smoothing term is smaller. Similar observations are found when the policy maker responds to the inflation measured in terms of the CPI.

As a final point it is worth noting that in this small open economy model, the information available to the policy maker is insufficient to eliminate all welfare losses even in Case 1 where the central bank is assumed to be able to observe all contemporaneous market values. Hence even in that case, the combination of sticky prices, incomplete asset markets, and incomplete exchange rate pass allows interest-rate smoothing to improve policy performance. ${ }^{94}$

\footnotetext{
${ }^{94}$ In an earlier version of this thesis, a small open economy model with complete exchange rate passthrough and complete asset markets. In that case the central bank that could access all contemporaneous information could calculate perfectly the contemporary value of the Wicksellian natural rate that when used resulted domestic prices becoming completely stabilized and the output gap eliminated. In this case the welfare loss could be driven to zero. In this case, however, requiring the monetary authority to use interestrate smoothing as an additional term in the policy rule reduced policy performance. This is consistent with our suggestion that it is only when there is information missing to the policy maker that the lagged interest rate add to policy performance and lower welfare losses.
} 
Table 6.19

The welfare gains from incorporating the lagged interest rate

\begin{tabular}{l|l|l|l|l|l}
\hline \multirow{2}{*}{$\begin{array}{l}\text { Information } \\
\text { cases }\end{array}$} & \multicolumn{5}{|c}{$W L_{S} / W L_{N}{ }^{*}$} \\
\cline { 2 - 6 } & Rule 1 (C) & Rule 2 (EX) & Rule 3 (PT) & Rule 4 (TR) & Rule 5 (IT) \\
\hline Case 1 & 0.9226 & 0.9205 & 0.9210 & 0.9201 & 0.9119 \\
\hline Case 2 & 0.8291 & 0.8296 & 0.8291 & 0.8306 & 0.8174 \\
\hline Case 3 & 0.9818 & 0.9654 & 0.9682 & 0.9654 & 0.9654 \\
\hline Case 4 & 0.6818 & 0.6730 & 0.6811 & 0.6505 & 0.6397 \\
\hline
\end{tabular}

A completely different reason why interest rate smoothing works to improve welfare performance in our analysis is because the central bank seeks to smooth interest rates as an entirely separate policy goal, that is, in addition to its inflation and output gap objectives. Here it is important to recognize that in the literature generally, it is necessary to add the squared change in the interest rate term $\left(\hat{R}_{t}-\hat{R}_{t-1}\right)^{2}$ arbitrarily into a standard loss function to derive results that mimic central bank interest rate smoothing behavior (see, for example, Rudebusch and Svensson, 1999; and Rudebusch, 2002). In our model, however, interest rate variability arises as a separate argument in the linearization of the welfare function because of the presence of the real money balances in the utility function. ${ }^{95}$ The importance of the real balance effect for interest rate smoothing can be found in simulations that indicate that when the weight on the interest gap in the loss function is reduced to zero, interest-rate smoothing policy rules cannot always deliver superior policy performance. In some cases, the addition of interest rate smoothing now has no affect. For example, in Case 1 the welfare loss under Rule 2 when the central bank

\footnotetext{
${ }^{95}$ This suggests that the current practice of ignoring a motivation for money use in models of this type (by leaving money balances out of the utility function, for example) does have a cost when it comes to implementing the analysis.
} 
targets the domestic output price is 0.0048 , while it is also 0.0048 after adding smoothing term. Similarly, the welfare losses remain identical under Rule 2 when the central bank responds to the CPI price. Similar results can be found for Cases 3 and 4.

Our simulations for the other information cases suggest a few other points that deserve mention. First, when the constraint on the variation in interest rate gap is removed from the loss function (as done above), the value of the optimized reaction coefficient on inflation becomes much larger than when the constraint was there. This shows not only a re-weighting of the policy targets but also that a concern with interest rate variability does not now constrain the central bank from reacting more strongly to innovations impacting the economy. The monetary authority becomes more aggressive. Second, when the simple rules are not optimized, the interest-rate smoothing rules can still deliver better performance. This again suggests an information role for lagged interest rates. That is, to the extent the monetary authority foregoes the gain available from optimization, there is a potential gain that the use of lagged interest rates may allow being realized. This is most apparent in Cases 3 and 4 . There the policy rules with inertia always generate better policy performance outcomes even when the simple rules are not optimized.

A final reason given for why interest rate smoothing improves the performance of a simple optimal rule is because it allows for the capture of the persistence of shocks. Aoki (2003), for example, argues that the reason why a optimal simple rule should involve a lagged interest rate term is because it is optimal for the central bank to respond to the persistence of economic shocks impacting the economy. In his model, then, the degree of interest-rate smoothing depends directly on the degree of persistence of demand 
shocks. This implies that if no persistence arose in the model, then policy performance could not be improved by adding lagged interest rate term into the optimal simple policy rule. To test whether this hypothesis is applicable to our analysis, we re-simulated the model for the case of no persistence to see whether welfare losses would still fall with interest rate smoothing? In our framework, there are four shocks, each of which follows an AR (1) process with persistence. In addition, the presence of price indexation leads any inflation rate shock to persist to some extent. Hence to shut off the persistence of the model, we set $\rho_{A}=\rho_{1}=\rho_{2}=\rho_{y}=\delta=0$. The test results show the opposite. First, optimal simple rules with interest-rate smoothing still produce better welfare outcomes; but even more interestingly, the less persistence there is in the model, the larger the welfare loss and the larger (rather than smaller) welfare gain from using the interest rate smoothing. That is, while there appear to be more than one reason for why interest rate smoothing produces better policy performance in our analysis, one of those reasons is not that it allows for the better incorporation of the model's persistence in the money rule.

\subsection{Concluding Remarks}

At present the Taylor rule is the monetary policy rule most often advocated in the literature, even for the case of a small open economy subject to external shocks. It was also the money rule that performed best in our small open economy model when the reaction coefficients were simply assigned as the values most often used in the literature. However, the conjecture that the Taylor rule will continue to perform best when the reaction coefficients are found optimally has proved misleading if not entirely wrong. In our model, it is always optimal to augment the Taylor rule with some factor that allows 
for the incorporation of external complications - in our case either the exchange rate gap and/or the law of one price gap. This finding is robust across our four different cases of information availability.

The choice of the appropriate price level and/or inflation rate to target has been another interesting policy question. While it appears that most inflation-targeting central banks have chosen to measure the rate of inflation in terms of some version of the CPI, our analysis finds that despite the appearance of the consumer price index is an important variable within the model, a central bank that bases its policy rule on domestic output prices will normally perform better overall than if it focuses on a consumer price index. This policy prescription is not unique to this model but has been also found by Clarida et al $(2001)^{96}$. As such our analysis reinforces this policy prescription.

Third, the addition of the lagged interest rate to an optimal simple policy rules typically enhances the level of social welfare and hence policy performance. The reason why this is the case was explored at length and appears to be for a combination of reasons - its implicit ability to capture information missed in other policy instruments, the fact that welfare depends directly in interest rate variability, and as a way of establishing and maintaining commitment. Perhaps most controversially, we find in section 6.3 that the optimized value of the coefficient on the lagged interest rate is greater than one. This is completely different from the empirical estimate usually found in the literature where they most often range between 0.7 and 0.9 . To the extent that this model captures some of the more important features of small open economies, it would imply that the central banks of these economies have been too timid in practice.

\footnotetext{
${ }^{96}$ Clarida et al. find that the central bank should target domestic inflation and allow the exchange rate to float when there is perfect exchange rate pass-through.
} 
Finally, our analysis suggests that the marginal welfare gain from incorporating foreign exchange considerations and/or lagged interest rates falls as the relevant information available to the policy maker improves. This is perhaps our most practical policy finding. It suggests that as a practical proposition, the less well developed is the statistical information available to a small open economy, the larger will be the gain from including some measure of the size of the external shock facing the economy and/or lagged interest rates in the monetary policy rule. 


\section{Appendix to Chapter 6}

\section{A6. 1 Partially optimal simple policy rules with inertia}

Tables A6.1 through A6.3 summarized the simulation results in Cases 2, 3, and 4 when alternative partially optimal simple money rules are applied. And note that here inflation targeted by the monetary authority is measured in CPI prices.

\section{Table A6.1}

Case 2: Partially optimal inertial simple rules using CPI prices

$$
\hat{R}_{t}=\phi_{\pi} \hat{\Pi}_{t}+\phi_{x} \hat{Y}_{t}+\phi_{q} \hat{q}_{t}+\phi_{\Psi} \hat{\Psi}_{t}+0.8 \hat{R}_{t-1}
$$

\begin{tabular}{c|c|c|c|c|c}
\hline \multirow{2}{*}{ Policy Rule } & \multicolumn{3}{|c|}{ Optimized reaction coefficients } & \multirow{2}{*}{ Welfare Loss } \\
\cline { 2 - 5 } & $\phi_{\pi}$ & $\phi_{x}$ & $\phi_{q}$ & $\phi_{\psi}$ & \\
\hline 1 (C) & 6.9719 & 0.1513 & 0.0616 & -0.2031 & 0.2111 \\
\hline 2 (EX) & 7.9511 & 0.3260 & -0.1645 & 0 & 0.2124 \\
\hline 3 (PT) & 7.1138 & 0.1851 & 0 & -0.1817 & 0.2112 \\
\hline 4 (TR) & 7.7745 & 0.2574 & 0 & 0 & 0.2128 \\
\hline 5 (IT) & 14.9898 & 0 & 0 & 0 & 0.2207 \\
\hline
\end{tabular}

Table A6.2

Case 3: Partially optimal inertial simple rules using CPI prices

$$
\hat{R}_{t}=\phi_{w} \hat{r}_{t-1}^{f}+\phi_{\pi} \hat{\Pi}_{t-1}+\phi_{x} x_{t-1}+\phi_{q} q_{g t-1}+\phi_{\Psi} \hat{\Psi}_{t-1}+0.8 \hat{R}_{t-1}
$$

\begin{tabular}{c|c|c|c|c|c|c}
\hline \multirow{2}{*}{$\begin{array}{c}\text { Policy } \\
\text { Rule }\end{array}$} & \multicolumn{4}{|c|}{ Optimized reaction coefficients } & \multirow{2}{*}{ Welfare Loss } \\
\cline { 2 - 6 } & $\phi_{w}$ & $\phi_{\pi}$ & $\phi_{x}$ & $\phi_{q}$ & $\phi_{\psi}$ & \\
\hline $1(\mathrm{C})$ & 0.5613 & 1.3725 & -0.0752 & 0.1137 & -0.0703 & 0.1797 \\
\hline
\end{tabular}




\begin{tabular}{c|c|c|c|c|c|c}
\hline 2 (EX) & 0.7053 & 1.9798 & -0.0266 & -0.015 & 0 & 0.1808 \\
\hline 3 (PT) & 0.6419 & 1.8591 & -0.0304 & 0 & -0.0191 & 0.1805 \\
\hline 4 (TR) & 0.7268 & 1.9875 & -0.0358 & 0 & 0 & 0.1809 \\
\hline 5 (IT) & 0.06022 & 1.6035 & 0 & 0 & 0 & 0.1811 \\
\hline
\end{tabular}

Table A6.3

Case 4: Partially optimal inertial simple rules using CPI prices

$$
\hat{R}_{t}=\phi_{\pi} \hat{\Pi}_{t-1}+\phi_{x} \hat{Y}_{t-1}+\phi_{q} \hat{q}_{t-1}+\phi_{\Psi} \hat{\Psi}_{t-1}+0.8 \hat{R}_{t-1}
$$

\begin{tabular}{c|c|c|c|c|c}
\hline \multirow{2}{*}{ Policy Rule } & \multicolumn{4}{|c|}{ Optimized reaction coefficients } & Welfare Loss \\
\cline { 2 - 5 } & $\phi_{\pi}$ & $\phi_{x}$ & $\phi_{q}$ & $\phi_{\psi}$ & \\
\hline 1 (C) & 3.3381 & 0.0614 & 0.0213 & -0.1557 & 0.2163 \\
\hline 2 (EX) & 3.5157 & 0.1928 & -0.1752 & 0 & 0.2204 \\
\hline 3 (PT) & 3.3547 & 0.0721 & 0 & -0.1479 & 0.2163 \\
\hline 4 (TR) & 3.4118 & 0.1283 & 0 & 0 & 0.2229 \\
\hline 5 (IT) & 4.9415 & 0 & 0 & 0 & 0.2336 \\
\hline
\end{tabular}

\section{A6. 2 Non-inertial optimal rules}

Table A6.4 through A6.6 report the simulation results when non-inertial optimal simple policy rules are employed in Cases 2, 3 and 4, respectively. Again, the CPI prices are used here.

\section{Table A6.4}

\section{Case 2: Optimal non-inertial simple money rules in CPI prices}

$$
\hat{R}_{t}=\phi_{\pi} \hat{\Pi}_{t}+\phi_{x} \hat{Y}_{t}+\phi_{q} \hat{q}_{t}+\phi_{\Psi} \hat{\Psi}_{t}
$$

\begin{tabular}{c|c|c|c|c|c}
\hline \multirow{2}{*}{ Policy Rule } & \multicolumn{4}{|c|}{ Optimized reaction coefficients } & \multirow{2}{*}{ Welfare Loss ** } \\
\cline { 2 - 5 } & $\phi_{\pi}{ }^{*}$ & $\phi_{x}$ & $\phi_{q}$ & $\phi_{\psi}$ & \\
\hline 1 (C) & 10 & 0.3174 & 0.1879 & -0.1369 & $0.2354(0.2140)$ \\
\hline
\end{tabular}




\begin{tabular}{c|c|c|c|c|c}
\hline 2 (EX) & 10 & 0.4028 & 0.0624 & 0 & $0.2356(0.2146)$ \\
\hline $3($ PT) & 10 & 0.4100 & 0 & -0.0617 & $0.2356(0.2141)$ \\
\hline 4 (TR) & 10 & 0.4269 & & & $0.2357(0.2151)$ \\
\hline 5 (IT) & 10 & 0 & 0 & 0 & $0.2516(0.2219)$ \\
\hline
\end{tabular}

Note that the interpretation of notation * and ** is the same as Table 6.12 .

\section{Table A6.5}

\section{Case 3: Optimal non-inertial simple rules in CPI prices}

$$
\hat{R}_{t}=\phi_{w} \hat{r}_{t-1}^{f}+\phi_{\pi} \hat{\Pi}_{t-1}+\phi_{x} x_{t-1}+\phi_{q} \hat{q}_{g, t-1}+\phi_{\Psi} \hat{\Psi}_{t-1}
$$

\begin{tabular}{c|c|c|c|c|c|c}
\hline \multirow{2}{*}{$\begin{array}{c}\text { Policy } \\
\text { Rule }\end{array}$} & \multicolumn{5}{|c|}{ Optimized reaction coefficients } & \multirow{2}{*}{ Welfare Loss } \\
\cline { 2 - 6 } & $\phi_{w}$ & $\phi_{\pi}$ & $\phi_{x}$ & $\phi_{q}$ & $\phi_{\psi}$ & \\
\hline 1 (C) & 1.3302 & 2.7548 & -0.2706 & 0.3612 & -0.2603 & 0.1866 \\
\hline 2 (EX) & 1.7992 & 4.5004 & 0.0090 & -0.1437 & 0 & 0.1899 \\
\hline 3 (PT) & 1.6605 & 4.1599 & -0.0761 & 0 & -0.0994 & 0.1884 \\
\hline 4 (TR) & 2.0056 & 4.6856 & -0.0717 & 0 & 0 & 0.1915 \\
\hline 5 (IT) & 2.0087 & 4.6838 & 0 & 0 & 0 & 0.1919 \\
\hline
\end{tabular}

Table A6.6

Case 4: Optimal non-inertial simple rules in CPI prices

$$
\hat{R}_{t}=\phi_{\pi} \hat{\Pi}_{t-1}+\phi_{x} \hat{Y}_{t-1}+\phi_{q} \hat{q}_{t-1}+\phi_{\Psi} \hat{\Psi}_{t-1}
$$

\begin{tabular}{c|c|c|c|c|c}
\hline \multirow{2}{*}{ Policy Rule } & \multicolumn{4}{|c|}{ Optimized reaction coefficients } & Welfare Loss \\
\cline { 2 - 5 } & $\phi_{\pi}$ & $\phi_{x}$ & $\phi_{q}$ & $\phi_{\psi}$ & \\
\hline 1 (C) & 20.8317 & 0.2012 & -0.2328 & -1.3779 & 0.2484 \\
\hline 2 (EX) & 14.6945 & 0.9500 & -1.4281 & 0 & 0.2580 \\
\hline 3 (PT) & 20.4254 & 0.0857 & 0 & -1.4488 & 0.2485 \\
\hline 4 (TR) & 8.8330 & 0.0994 & 0 & 0 & 0.2706 \\
\hline 5 (IT) & 11.2924 & 0 & 0 & 0 & 0.2790 \\
\hline
\end{tabular}




\section{Chapter 7}

\section{Optimal simple monetary policy rules when households observe aggregate prices with error}

\subsection{Introduction}

In the previous chapters we assumed that only the central bank faced a significant data deficiency when making its optimal policy decision. Private agents--both individuals and firms--were assumed to have perfect information in making their optimal choices. Our case for exploring the consequences of this asymmetry was that the decisions of private agents were somewhat less dependent on aggregate data than were the policy decisions of central government agencies such as the central bank. The problem, of course, is that private agents do need to know aggregate data to make their optimal consumption/production choices. For example, the firm needs to know the aggregate price level in order to set the appropriate (relative) price under Calvo pricing and knowledge of the current inflation rate is needed to adjust the current nominal price to indexation. In doing so, private individuals and firms share with the central bank the problem that aggregate indicators are not instantly available and, even when they are available with delay, such data are recognized to be preliminary and subject to later revision.

Hence from a conceptual point of view both private agents and the central bank encounter similar types of information problems and, if we take into account the superior information processing capabilities of the central bank, the policy maker may even have a 
comparative advantage in adapting to information constraints. It is then of interest to ask how our case for the incorporation of external considerations in optimal simple monetary policy rules is influenced by information issues that confront private agents. In particular, does the existence of individual measurement error weaken or strengthen the case for such rules.

Some cases of information uncertainty faced by private agents and/or policymakers have already been examined in the literature. For example, Orphanides and Williams (2005a, b) examine the performance and robustness of monetary policy rules in a macroeconomic model where the economy undergoes structural change and where both private agents and policymakers must relearn the true structure of the economy. Private agents then rely on adaptive learning to form expectations and update their beliefs based on incoming data. Policymakers employing an interest rate rule to maintain price stability and to minimize fluctuations of unemployment around its natural rate use a simple constant gain method to update their knowledge of the natural rate based on the observed rates of unemployment and ex post real interest rates. They find that efficient policies that take account of private learning and misperception of natural rates call for more aggressive response to inflation that would be optimal under perfect knowledge.

In the context of transitory rather than structural shocks, Svensson and Woodford (2003), following Backus and Driffill (1986) and Currie and Levine (1993), demonstrate that in the presence of forward-looking variables and symmetric partial information, the monetary policy response to the optimal estimates of the current output gap is the fundamentally the same as under certainty, that is, the certainty equivalence condition 
holds. Furthermore, the separation principle also applies in such an environment. ${ }^{97}$ They also show analytically that the optimal weight put on noisy indicator will depend upon the degree of uncertainty. In particular, when the degree of noise in the indicator of potential output is large, the optimal weight placed on the reaction coefficient of that indicator becomes small. This is slightly different from Orphanides' finding (2000). Orphanides argues that in such cases it is better to disregard uncertain data on the output gap in favour of placing greater reliance on inflation.

In a later extension of the earlier work discussed above, Svensson and Woodford (2004) show that when the private sector has more information than the policymaker, certainty equivalence still continues to hold when determining the optimal reaction function in state-space form, but not when the reaction function is in integrative form. In addition, the separation principle no longer holds. This is because the estimated state of the economy is no longer independent of the policy optimization process.

In this chapter we consider the consequences for optimal simple monetary policy rules of private agents having less than perfect price information (and often less accurate information than policymakers). Specifically, we begin by assuming that private agents can observe accurately their own individual price, the level of output, exchange and interest rates; but can observe the current aggregate levels of domestic and import prices only with error. Because all Calvo price setters are identical, measurement error is assumed to arise not as a consequence of structural change (as in Orphanides and

\footnotetext{
${ }^{97}$ It is well known that in the presence of partial information and there are no forward looking variables a linear model with a quadratic loss function is characterized by certainty equivalence. That is, the optimal policy is the same whether the state of the economy is fully observable (full information), or whether policy responds to an efficient estimate of the state vector rather than to its actual value. In addition, a separation principle applies. That is, the selection of the optimal policy (the optimization problem) and the estimation of the current state of the economy (the estimation or signal extraction problem) can be treated as separate problems. For more detail, see Chow (1975).
} 
Williams) but in response to transitory relative price shocks specific to each individual product that net out in the aggregate but create obstacles to accurate aggregate price measurement in the short run. In this context we examine how optimal simple policy rules change and assess whether the optimal coefficients in each policy rule require either a weakening/strengthening of response or a substitution away from noisy indicators to those that can be measured more accurately. To initiate this discussion we first assume that policymakers can fully access current information when making their policy decision. Hence we begin with an information structure that is explicitly asymmetric. In the later sections of the chapter, we consider the implications of both private agents and the monetary authority encountering the same measurement problem. What is of interest throughout is whether the optimal simple money rule continues to require some form of external measure in the optimal rule.

The rest of the chapter is organized as follows. The section 2 re-derives the model to incorporate measurement error. In section 3, we illustrate and discuss the simulation results that will follow these adjustments for policy rules with and without interest rate smoothing. Section 4 extends the measurement issue to the monetary authority. The last section presents concluding remarks.

\subsection{The Model of a Small Open Economy with Private Measurement Error}

The analytic framework in this extension remains fundamentally unchanged from earlier chapters except for the revised information structure. Hence to briefly review its central features, the world economy is made up of two regions: a domestic economy and a foreign one where the domestic economy is assumed to be so small that it has no impact 
on the foreign one. The domestic economy is represented by a representative household and a large number of identical firms. The government behavior is not modeled explicitly, except to collect taxes and transfer tax revenues to private agents (to offset monopolistic production). Households consume both domestic goods and imported goods by supplying labor in markets that are assumed to be perfectly competitive. Households are also assumed to be able to allocate consumption over time by accessing a one-period risk-free domestic bond and a risky foreign bond denominated in foreign currency. Asset markets are incomplete. Firms produce differentiated goods under monopolistic competition and price rigidities arise because firms set domestic good prices using Calvo's (1983) pricing specification and set imported good prices subject to quadratic adjustment costs--the latter leading to incomplete exchange rate pass-through. The inefficiency caused by monopolistic competition is offset by an optimal subsidy paid to firms, while inefficiency arising from sticky prices is addressed by policy makers through monetary policy. ${ }^{98}$ Foreign agents are modeled as identical to their domestic counterparts.

What is new to the analysis is that individuals and firms are assumed to observe some variables with error. Specifically, private agents are assumed to be able to observe their level of output, the exchange rate, and the interest rate accurately, but can observe the current period domestic price level $P_{H, t}$ and the current period import goods prices

\footnotetext{
${ }^{98}$ In the previous analysis there was no need to distinguish between a competitive market outcome under price clearing and economic efficiency. Private and social costs coincide once the monopoly distortion is removed. With measurement error, however, there is some ambiguity about which private outcome represents the efficient outcome. In what follows we assume that since utility is based on what households observe the real value of their money holdings to be (rather than on the actual level of their money holdings), then the efficient level of output will be the competitive market clearing outcome conditional on the price levels observed by the households. That is, the monetary authority is not expected to correct for the mistake that households make when observing aggregate prices.
} 
$P_{F, t}$ only with error. We motivate this form of measurement error in the following way. In our multi-market economy, individual producers are assumed to observe all past prices plus the prices they set this period costlessly. On the other hand, the current prices being set in other spot markets are assumed to be costly to observe such that accurate aggregate information becomes available only with delay. In previous chapters, this short run information cost introduced no measurement problem since all agents setting prices were identical and this knowledge implied accurate knowledge of the behavior of all other price setters. Each price setter could deduce accurately the aggregate price level arising each period by simple projection of their own choice. Hence to generate a short run measurement problem, we now assume that each firm is subject to a firm-specific shock, a relative shock arising in addition to the four aggregate shocks that already impact the model. Hence knowledge that the relative shock will be unique to the particular firm keeps the Calvo price setter from being able to project equal price adjustment to all other current price setters. In this way, Calvo pricing will result in a distribution of newly set prices that will reflect somewhat the relative shock arising in that market. It then follows that because information is costly to collect in the short run, any agency designed to estimate aggregate short run information from a sample of firm price settings and/or individual firms formulating a measure of the aggregate price level on the basis of the short run prices they encounter are likely to include random measurement error. Thus despite the fact that aggregation over all firm prices will result in an aggregate price level that will exclude the relative demand shock, the inability to observe all relative prices costlessly means that measurement error will creep into price setting behavior. The same 
situation is assumed to arise in the markets for foreign goods leading to the index of foreign prices also being observed with error.

The aggregate prices observed are denoted as $P_{H, t}{ }^{0}$ and $P_{F, t}{ }^{0}$ respectively. Because the measurement error exists only in the current period, households and firms observe historical prices accurately. This implies that $P_{H, t-k}{ }^{0}=P_{H, t-k}$ and $P_{F, t-k}{ }^{0}=P_{F, t-k}$ for $k \geq 1$. On the other hand, the policy maker is assumed to be able to access all information accurately. In this sense the initial model involves asymmetric information. $^{99}$

As before, the representative household maximizes the expected value of the discounted sum of time separable utilities

$$
U_{0}=E_{0} \sum_{t=0}^{\infty} \beta^{t} u\left(\frac{\chi_{1 t}}{1-\sigma} C_{t}^{1-\sigma}+\frac{1}{1-\varsigma}\left(\frac{M_{t}}{P_{t}^{0}}\right)^{1-\varsigma}-\frac{1}{1+\mu} \int_{0}^{1}\left(N_{t}(i)\right)^{1+\mu} d i\right)
$$

subject to an intertemporal budget constraint

$$
\begin{aligned}
& C_{t}+\frac{M_{t}}{P_{t}^{0}}+\frac{B_{t}}{P_{t}^{0} R_{t}}+\frac{\varepsilon_{t} B_{t}^{*}}{P_{t}^{0} R_{t}^{*} \exp \left(-b N F A_{t}^{0}+\ln \chi_{2 t}\right)}+\frac{T_{t}}{P_{t}^{0}}= \\
& \frac{1}{P_{t}^{0}} \int_{0}^{1} N_{t}(i) W_{t}(i) d i+\frac{1}{P_{t}^{0}} \int_{0}^{1} D_{t}(i) d i+\frac{1}{P_{t}^{0}} \int_{0}^{1} D_{t}(z) d z+\frac{M_{t-1}}{P_{t}^{0}}+\frac{B_{t-1}}{P_{t}^{0}}+\frac{\varepsilon_{t} B_{t-1}}{P_{t}^{0}}
\end{aligned}
$$

where notation is the same as in Chapter 2 , except that the consumer price index $P_{t}^{0}$ observed by households is defined as

$$
P_{t}^{0}=\left[(1-\alpha)\left(P_{H, t}^{0}\right)^{1-\eta}+\alpha\left(P_{F, t}^{0}\right)^{1-\eta}\right]_{\frac{1}{1-\eta}}
$$

99 This raises the question why the monetary authority does not simply pass this its superior information along to households and firms. The assumed inability to do so in our analysis implies the existence of credibility or timing (cost) issues in the transmission of reliable timely information to individuals. Hence at the time, individuals believe that they see the aggregate price level accurately and recognize their error with a one period lag. 
Similarly, the observed value of net foreign assets, $N F A_{t}{ }^{0}=\varepsilon_{t} B_{t}{ }^{*} / P_{t}^{0}$.

Solving the household's revised utility maximization problem we obtain the following first order conditions,

$$
\begin{gathered}
C_{t}^{-\sigma}=\beta R_{t} E_{t}\left(\frac{P_{t}^{0}}{P_{t+1}^{0}}\right)\left(\frac{\chi_{1 t+1}}{\chi_{1 t}}\right) C_{t+1}^{-\sigma} \\
C_{t}^{-\sigma}=\beta R_{t}^{*} \exp \left(-b N F A_{t}^{0}+\ln \chi_{2 t}\right) E_{t}\left(\frac{\varepsilon_{t+1}}{\varepsilon_{t}}\right)\left(\frac{P_{t}^{0}}{P_{t+1}^{0}}\right)\left(\frac{\chi_{1 t+1}}{\chi_{1 t}}\right) C_{t+1}{ }^{-\sigma} \\
\left(\frac{M_{t}}{P_{t}^{0}}\right)^{-\varsigma} / \chi_{1 t} C_{t}^{-\sigma}=\frac{R_{t}-1}{R_{t}} \\
\frac{N_{t}^{\mu}}{\chi_{t t} C_{t}^{-\sigma}}=\frac{W_{t}}{P_{t}^{0}} \\
R_{t}=R_{t}^{*} \exp \left(-b N F A_{t}^{0}+\ln \chi_{2 t}\right) \frac{\varepsilon_{t+1}}{\varepsilon_{t}}
\end{gathered}
$$

Turning to the supply side of the economy, each (identical) firm first solves the following cost minimization problem by choosing labor input given a certain level of output,

$$
\min _{N_{t}(i)}\left(\frac{W_{t}(i)}{P_{H, t}^{0}}\right) N_{t}(i)+\varphi_{H, t}(i)\left(Y_{t}(i)-A_{t} N_{t}(i)\right)
$$

This results in the firm's marginal cost function $\varphi_{H, t}(i)=W_{t}(i) / A_{t} P_{H, t}{ }^{\circ}$. Assuming a competitive (observable) labor market, this condition can be rewritten as $\varphi_{H, t}=W_{t} / A_{t} P_{H, t}{ }^{0}$. The firm then sets its price using Calvo pricing with indexation. The privately optimal price becomes, 


$$
P_{H, t}^{n}=\frac{E_{t} \sum_{k=t}^{\infty}(\omega \beta)^{k-t} \chi_{t+k} C_{t+k}{ }^{-\sigma} Y_{t+k}\left(P_{t+k}^{0}\right)^{-1}\left(P_{H, t+k}^{0}\right)^{1+\theta} X_{H, t t}{ }^{-\delta \theta} \varphi_{H, t+k}}{E_{t} \sum_{k=t}^{\infty}(\omega \beta)^{k-t} \chi_{t+k} C_{t+k}{ }^{-\sigma} Y_{t+k}\left(P_{t+k}^{0}\right)^{-1}\left(P_{H, t+k}^{0}\right)^{\theta} X_{H, t k}{ }^{\delta(1-\theta)}}
$$

Compared to the domestic producer, the representative importer can change its price, but only subject to a quadratic adjustment cost. The importer then sets its price $P_{F, t}(z)$ by solving the following maximization problem,

$$
\max E_{t} \sum_{k=0}^{\infty}\left\{Q_{t, t+k}\left[\left(1+\tau_{M}\right) P_{F, t+k}(z)-\varepsilon_{t} P_{F, t+k}{ }^{*}(z)\right] C_{F, t+k}(z)-A C_{M, t+k} P_{F, t+k}{ }^{0} C_{F, t+k}\right\}
$$

where

$$
C_{F, t}(z)=\left(\frac{P_{F, t}(z)}{P_{F, t}{ }^{\theta}}\right)^{-\theta} C_{F, t} \text { and } A C_{M, t}=\frac{\omega_{M}}{2}\left(\frac{P_{F, t}(z) / P_{F, t-1}(z)}{\left(P_{F, t-1}{ }^{0} / P_{F, t-2}{ }^{0}\right)^{\delta}}-1\right)^{2}
$$

Solving the problem and log-linearizing the relevant first-order conditions we obtain the following equations that describe the small open economy model with measurement errors.

$$
\begin{aligned}
& {\left[1+\frac{\gamma_{n}(\phi+\alpha-1)}{1-\alpha}\right] \hat{\Pi}_{H, t}=\frac{\omega \delta}{1-\omega \beta(1-\omega-\omega \delta)} \hat{\Pi}_{H, t-1}} \\
& +\frac{\omega \beta}{1-\omega \beta(1-\omega-\omega \delta)} E_{t} \hat{\Pi}_{H, t+1}+\frac{\gamma_{n}(\mu+\sigma-\mu \alpha)}{1-\alpha} \hat{Y}_{t} \\
& -\frac{\gamma_{n}(\phi+\alpha-1)}{(1-\alpha)^{2}} \hat{q}_{t}+\frac{\gamma_{n} \alpha(\sigma \eta+\alpha-1)}{(1-\alpha)^{2}} \hat{\Psi}_{t} \\
& +\left[\frac{\gamma_{n}(\phi+\alpha-1)}{1-\alpha}+\frac{(1-\omega)(1-\omega \beta-\omega \beta \delta)}{1-\omega \beta(1-\omega-\omega \delta)}\right] \hat{\Pi}_{H, t}{ }^{0} \\
& +\frac{\gamma_{n} \alpha(\sigma \eta-\phi)}{(1-\alpha)^{2}}\left(\hat{\Pi}_{F, t}-\hat{\Pi}_{F, t}{ }^{0}\right)-\frac{\gamma_{n} \alpha \sigma}{1-\alpha} \hat{Y}_{t}^{*}-\gamma_{n}(1+\mu) \hat{A}_{t}-\gamma_{n} \hat{\chi}_{1 t}
\end{aligned}
$$

where $\gamma_{n}=\frac{(1-\omega)(1-\omega \beta)}{1+\omega \beta(1-\omega-\omega \delta)}$ and $\phi=[\alpha(2-\alpha)(\sigma \eta-1)+1]$. 


$$
\begin{gathered}
\hat{\Pi}_{F, t}=\delta \hat{\Pi}_{F, t-1}+\beta E_{t} \hat{\Pi}_{F, t+1}-\beta \delta \hat{\Pi}_{F, t}{ }^{0}+\frac{\theta}{\omega_{M}} \hat{\Psi}_{t}, \\
\hat{Y}_{t}=E \hat{Y}_{t+1}-\frac{1-\alpha}{\sigma}\left(\hat{R}_{t}-(1-\alpha) E_{t} \hat{\Pi}_{H, t+1}-\alpha \hat{\Pi}_{F, t+1}\right) \\
+\frac{\alpha \eta(2-\alpha)}{1-\alpha}\left(\hat{q}_{t}-E_{t} \hat{q}_{t+1}\right)-\frac{\sigma \eta}{1-\alpha}\left(\hat{\Psi}_{t}-E_{t} \hat{\Psi}_{t+1}\right) \\
+\frac{\phi}{\alpha}\left(\hat{\Pi}_{H, t}-\hat{\Pi}_{H, t}{ }^{0}\right)+\frac{\phi \alpha-\alpha \sigma \eta}{\sigma(1-\alpha)}\left(\hat{\Pi}_{F, t}-\hat{\Pi}_{F, t}{ }^{0}\right) \\
+\alpha\left(\hat{Y}_{t}^{*}-\hat{Y}_{t+1}{ }^{*}\right)+\frac{1-\alpha}{\sigma}\left(\hat{\chi}_{1 t}-\hat{\chi}_{1 t+1}\right) \\
\hat{q}_{t}=E_{t} \hat{q}_{t+1}+\frac{\sigma(1-\alpha)}{\phi}\left(\hat{Y}_{t}-E_{t} \hat{Y}_{t+1}\right)+\frac{\alpha \sigma \eta}{\phi}\left(\hat{\Psi}_{t}-E_{t} \hat{\Psi}_{t+1}\right) \\
-(1-\alpha)\left(\hat{\Pi}_{H, t}-\hat{\Pi}_{H, t}{ }^{0}\right)-\left[\alpha-\frac{\alpha \sigma \eta}{\phi}\right]\left(\hat{\Pi}_{F, t}-\hat{\Pi}_{F, t}{ }^{0}\right) \\
-\frac{b(1-\alpha)^{2}}{\phi} N F A_{t}+\frac{(1-\alpha)^{2}}{\phi} \hat{\chi}_{2 t}-\frac{\sigma(1-\alpha)}{\phi}\left(\hat{Y}_{t}^{*}-E_{t} \hat{Y}_{t+1}^{*}\right) \\
N F A_{t}=\frac{1}{\beta} N F A_{t-1}-\frac{\alpha}{\beta(1-\alpha)} \hat{Y}_{t}+\frac{\alpha[\eta(2-\alpha)+\alpha-1]}{\beta(1-\alpha)^{2}} \hat{q}_{t} \\
-\frac{\alpha(\eta+\alpha-1)}{\beta(1-\alpha)^{2}} \hat{\Psi}_{t}+\frac{\alpha \eta(2-\alpha)}{\beta(1-\alpha)}\left(\hat{\Pi}_{H, t}-\hat{\Pi}_{H, t}{ }^{0}\right) \\
+\frac{\alpha \eta[\alpha(2-\alpha)-1]}{\beta(1-\alpha)^{2}}\left(\hat{\Pi}_{F, t}-\hat{\Pi}_{F, t}{ }^{0}\right)+\frac{\alpha}{\beta(1-\alpha)} \hat{Y}_{t}^{*} \\
\hat{\Psi}_{t}=\hat{\Psi}_{t+1}+(1-\alpha) \hat{\Pi}_{H, t}-(1-\alpha) \hat{\Pi}_{F, t}+\hat{q}_{t}-\hat{q}_{t-1},
\end{gathered}
$$

Here equation (7.12) is the revised New Keynesian Phillips curve while equation (7.13) describes the new equation of motion for import price inflation. The new equation for aggregate demand is expressed in equation (7.14). The last three equations then represent the revised versions of the real exchange rate equation, the net foreign asset position equation and the law of one price gap. By inspection it can be seen that the key gaps in the problem now also depend on the difference between observed and actual aggregate prices. Furthermore, it is easy to verify that under the conditions that 
$\hat{\Pi}_{H, t}=\hat{\Pi}_{H, t}{ }^{0}$ and $\hat{\Pi}_{F, t}=\hat{\Pi}_{F, t}{ }^{0}$, the six equations are identical to their corresponding counterparts in Chapter 2.

In the previous chapters we assumed that an optimal subsidy was used to offset the only inefficiency in the model (arising from monopolistic competition). Therefore the natural rate of output, $\hat{Y}_{t}^{f}$, the natural rate of the real exchange rate, $\hat{q}_{t}^{f}$, and the Wicksellian natural interest rate, $\hat{r}_{H, t}^{f}$, are efficient (welfare maximizing) outcomes. Under the current information structure, however, there is a discrepancy between the price that is observed by private agents (and acted upon) and the price that clears the market under competitive conditions. Hence under the assumption that what matters to households is the (observed) price level in their utility function, the natural rate of output is now expressed as

$$
\begin{aligned}
& \hat{Y}_{t}^{f}=\frac{\phi+\alpha-1}{(\mu+\sigma-\mu \alpha)(1-\alpha)} \hat{q}_{t}^{f}+\frac{\phi+\alpha-1}{(\mu+\sigma-\mu \alpha)(1-\alpha)}\left[\left(\hat{\Pi}_{H, t}\right)^{f}-\left(\hat{\Pi}_{H, t}{ }^{0}\right)^{f}\right] \\
& -\frac{\alpha(\sigma \eta+\alpha-1)}{(\mu+\sigma-\mu \alpha)(1-\alpha)}\left[\left(\hat{\Pi}_{F, t}\right)^{f}-\left(\hat{\Pi}_{F, t}{ }^{0}\right)^{f}\right]+\frac{\alpha \sigma}{\mu+\sigma-\mu \alpha} \hat{Y}_{t}^{*} \\
& +\frac{(1+\mu)(1-\alpha)}{\mu+\sigma-\mu \alpha} \hat{A}_{t}+\frac{1-\alpha}{\mu+\sigma-\mu \alpha} \hat{\chi}_{1 t}
\end{aligned}
$$

where

$$
\hat{\Pi}_{H, t}^{f}=\frac{1}{\alpha}\left[\begin{array}{c}
\frac{\mu+\sigma-\mu \alpha}{1-\alpha}\left(\hat{Y}_{t}^{f}-\hat{Y}_{t-1}{ }^{f}\right)-\frac{\alpha \sigma \eta(2-\alpha)}{(1-\alpha)^{2}}\left(\hat{q}_{t}^{f}-\hat{q}_{t-1}{ }^{f}\right) \\
-(1+\mu)\left(\hat{A}_{t}-\hat{A}_{t-1}\right)-\left(\hat{\chi}_{1 t}-\hat{\chi}_{1 t-1}\right)-\frac{\alpha \sigma}{(1-\alpha)}\left(\hat{Y}_{t}^{*}-\hat{Y}_{t-1}{ }^{\prime \prime}\right)
\end{array}\right]
$$

Previously under complete asset markets and completely flexible import prices, the law of one price held for import goods so that $\hat{\Pi}_{F, t}^{f}=0$. However, when import prices are not observed accurately by private agents, $\left(\hat{\Pi}_{F, t}{ }^{0}\right)^{f}$ is not zero. Its value will 
depend upon how $P_{F, t}{ }^{0}$ is specified (explained more fully below). Similarly, the real exchange rate under perfectly flexible (but inaccurately measured) prices is

$$
\hat{q}_{t}^{f}=\frac{\sigma(1-\alpha)}{\phi}\left(\hat{Y}_{t}^{f}-\hat{Y}_{t}^{*}\right)
$$

where the $\hat{Y}_{t}^{f}$ is as determined in (7.18). Finally, for the same definitions, the Wicksellian natural rate is

$$
\hat{r}_{H, t}{ }^{f}=\frac{\sigma}{1-\alpha} E_{t} \Delta \hat{Y}_{t+1}^{f}-\frac{\phi+\alpha-1}{(1-\alpha)^{2}} E_{t} \Delta \hat{q}_{t+1}{ }^{f}-\frac{\alpha \sigma}{1-\alpha} E_{t} \Delta \hat{Y}_{t+1}^{*}-E_{t} \Delta \hat{\chi}_{1 t+1}
$$

Thus far we have only assumed that private agents observe prices with error. The specific form of the measurement error has not yet been defined. We now specify the measurement error by assuming that private agents observe aggregate prices at time $t$ as equal to: $:^{100}$

$$
\begin{aligned}
& \hat{P}_{H, t}{ }^{0}=\hat{P}_{H, t}+\varepsilon_{P H, t}, \\
& \hat{P}_{F, t}{ }^{0}=\hat{P}_{F, t}+\varepsilon_{P F, t},
\end{aligned}
$$

where $\hat{P}_{H, t}{ }^{0}\left(\hat{P}_{H, t}\right)$ are the log deviation of observed (market clearing) prices of the domestic goods from its steady state value, and $\hat{P}_{F, t}{ }^{0}\left(\hat{P}_{F, t}\right)$ the log deviation of observed (market clearing) prices of the imported goods from its steady state value, while $\varepsilon_{P H, t}$ and $\varepsilon_{P F, t}$ denote measurement errors, which are assumed to be iid with a mean value of zero and constant variance equal to ${\sigma_{P H}}^{2}$ and ${\sigma_{P F}}^{2}$. Subtracting $\hat{P}_{H, t-1}$ on both sides of (7.21) and $\hat{P}_{F, t-1}$ on both sides of (7.22) We then obtain the following two expressions:

\footnotetext{
${ }^{100}$ In other contexts, this assumption has been used by Aoki (2003) and Svensson and Woodford (2003, 2004).
} 


$$
\begin{aligned}
& \hat{\Pi}_{H, t}{ }^{0}=\hat{\Pi}_{H, t}+\varepsilon_{P H, t}, \\
& \hat{\Pi}_{F, t}{ }^{0}=\hat{\Pi}_{F, t}+\varepsilon_{P F, t} .
\end{aligned}
$$

Under the perfectly flexible prices, equation (7.24) can be written as

$$
\left(\hat{\Pi}_{F, t}{ }^{0}\right)^{f}=\left(\hat{\Pi}_{F, t}\right)^{f}+\varepsilon_{P F, t}
$$

To investigate the policy implications of private agents acting on prices that contain measurement error, we need to specify the policy instrument and the welfare criteria used to evaluate the alternative policies. To make our simulation results comparable with the work done earlier, we assume that policy maker employs the same set of policy instruments as it did in previous chapters. On this basis we again evaluate five simple rules with and without interest rate smoothing. Rule 1 is our most comprehensive money rule, corresponding to the case where the monetary authority sets the short-term interest rate in response to changes in the inflation rate, the real GDP output gap, the real exchange rate gap, and the law of one price gap. This we call the comprehensive rule (C). Dropping the law of one price gap from Rule 1 gives us Rule 2, called the real exchange rate rule (ER). For comparative purposes, we replace the real exchange rate term in Rule 2 with the law of one price gap. This becomes Rule 3, the exchange rate pass-through rule (PT). Rule 4 has no openness component and corresponds to the Taylor rule (TR). Rule 5 further supposes that the monetary policy authority chooses to focus exclusively on inflation. This we call inflation targeting (IT). ${ }^{101}$ The welfare criterion used to evaluate the alternative monetary policy is taken from

\footnotetext{
101 The terminology used here differs somewhat from that used by Svensson (2000). Svensson links "targeting" to the monetary authority's objective. Hence when stabilizing inflation around the inflation rate target is the only objective for monetary policy, Svensson calls this strict inflation targeting. When there are additional objectives for monetary policy, for instance when the objective of stabilizing output is added, Svensson calls the resulting monetary policy rule flexible inflation targeting.
} 
Chapter 3 (and reflects the utility functions of individuals). Furthermore the values of the structural parameters are exactly the same as in Chapter 4. Finally to keep the analysis simple, we assume that the two measurement errors $\varepsilon_{P H, t}$ and $\varepsilon_{P F, t}$ are identical with ${\sigma_{P H}}^{2}={\sigma_{P F}}^{2}$ and ${\sigma_{P H}}^{2}={\sigma_{P F}}^{2}=0.005^{2}$

\subsection{Optimal Simple Policy Rules when Private Agents Observe Aggregate Price}

\section{Levels with Error}

As mentioned in section 7.1 , the main concern in this chapter is the impact on simple policy rules when households and firms optimize on less than accurate price information. To solve for these optimal rules, we first rewrite the model presented in preceding section compactly as

$$
A_{1}\left[\begin{array}{l}
X_{1, t+1} \\
E_{t} X_{2, t+1}
\end{array}\right]=A_{2}\left[\begin{array}{l}
X_{1, t} \\
X_{2, t}
\end{array}\right]+B R_{g, t}+\left[\begin{array}{l}
\varepsilon_{t+1} \\
0_{2}
\end{array}\right]
$$

where $X_{1, t}$ is a column vector of predetermined variables, and the initial values of $X_{1,0}$ are all givens. $X_{2, t}$, on the other hand, is a column vector of non-predetermined variables. $R_{g, t}$ is the gap between the current short-term interest rate and the Wicksellian natural rate and is the policy instrument. $\varepsilon_{t+1}$ is a column vector of innovations to the economic system and $0_{2}$ is a zero column vector. Lastly, $A_{1}$ and $A_{2}$ are matrices, and $B$ a column vector of the structural parameters, all of which are assumed to be constants.

Assume then that the monetary authority can precommit to a simple policy rule of the form $\hat{R}_{t}=-F X_{t}$, where $\hat{R}_{t}$ is the policy instrument, $F$ is a $1 \times n$ row vector constrained to be sparse in some specified way which depends upon the policy maker's 
preferences and $X_{t}=\left[X_{1 t}, X_{2 t}\right]$. The optimal simple rule will then solve for the values of $\mathrm{F}$ that minimize the loss function subject to the economic system denoted by $(7.26)$. The solutions are presented in Table 7.1 through 7.3.

The optimal simple policy rules and associated welfare losses summarized in Table 7.1 are based on the following assumption of policymaker behavior. When the economy is hit by shocks, key endogenous economic variables such as inflation and output are driven from their targeted levels (in our analysis these targets are defined in terms of their fully flexible price levels). The policymaker then adjusts its policy instrument (the shortterm interest rate) to bring these targeted variables back into line with their designated targets immediately (with no delay in response allowed). In cases where the interest rate adjusts only in response to the deviations of these variables from target, the policy rule is sometimes called a non-inertia policy rule (to distinguish it from rules that involve smoothing). Our general specification of this form of rule is

$$
\hat{R}_{t}=\phi_{w} \hat{r}_{H, t}^{f}+\phi_{\pi} \hat{\Pi}_{H, t}+\phi_{x} x_{t}+\phi_{q} q_{g, t}+\phi_{\Psi} \hat{\Psi},
$$

where $\hat{r}_{H, t}^{f}$ and $\hat{\Pi}_{H, t}$ are the interest and inflation rate gaps, and the output gap $x_{t}$ and the real exchange rate gap $q_{g, t}$ are defined, respectively, to be $x_{t}=\hat{Y}_{t}-\hat{Y}_{t}^{f}$, and $q_{g, t}=\hat{q}_{t}-\hat{q}_{t}^{f}$

In Chapter 6, our most important finding was that the first three policy rules, rules that incorporate either the exchange rate or the law of one price gap (or both), worked at least as well as, and often much better than, rules that exclude external considerations. This finding is observed in Table 7.1 as well. The welfare losses in the last column of the first three rows of Table 7.1 are all lower than the corresponding values for the Taylor 
Rule and Inflation Targeting Rule in rows four and five. Hence our previous conclusion that some reflection of the external side of the economy should be incorporated into the policy rule is robust to this specification of measurement error. However, the similarity of this general finding does not mean that the asymmetric information structure has had no impact on the optimal simple policy rules. Rather, when we compare the results summarized in Table 7.1 with those in Table $7.2^{102}$ (in which private agents are assumed to access to perfect information), a number of features are worth discussing in more detail. First, note that as would be expected, noisy price signals mislead private agents and cause their "optimized decisions" to deviate further from the optimal choices that would be made when information is complete. Hence despite the actions of the central bank, the cost arising from noisy information is reflected in a higher welfare losses. Not only is the welfare loss larger under each of the money rules in Table 7.1 , but the minimum welfare loss reported in Table 7.2 rises from 0.1719 to 0.1721 in Table 7.1 . Second, note that the optimal coefficients are typically smaller when one compares the cells of Table 7.2 to the corresponding cells in Table 7.1. This coincides with the finding of Woodford and Svensson (2003) cited earlier, i.e., as information becomes less reliable (subject to greater noise) the weight placed on the reaction coefficient becomes smaller.

\section{Table 7.1}

Non-inertial optimal policy rules when inflation contains noise

\begin{tabular}{l|l|l|l|l|l|l}
\hline \multirow{2}{*}{$\begin{array}{l}\text { Policy } \\
\text { Rule }\end{array}$} & \multicolumn{5}{|c|}{ Optimized reaction coefficients } & \multirow{2}{*}{ Welfare Loss } \\
\cline { 2 - 6 } & $\phi_{w}$ & $\phi_{\pi}$ & $\phi_{x}$ & $\phi_{q}$ & $\phi_{\psi}$ & \\
\hline $1(\mathrm{C})$ & 1.8517 & 5.0377 & -0.0734 & 0.2827 & -0.2117 & 0.1721 \\
\hline $2(\mathrm{EX})$ & 2.9829 & 10.4648 & 0.1979 & -0.1385 & 0 & 0.1727 \\
\hline
\end{tabular}

102 Table 7.2 is reproduced from Table 6.13 , for convenience. Similarly Tables 7.4 and 7.6 are reproduced from Tables 6.1 and 6.9 , respectively. 


\begin{tabular}{l|l|l|l|l|l|l}
\hline 3 (PT) & 2.6372 & 8.9678 & 0.1116 & 0 & -0.0999 & 0.1724 \\
\hline 4 (TR) & 3.0581 & 10.3410 & 0.1134 & 0 & 0 & 0.1729 \\
\hline 5 (IT) & 3.1399 & 10.9175 & 0 & 0 & 0 & 0.1731 \\
\hline
\end{tabular}

Table 7.2

Non-inertial optimal policy rules when information is complete

\begin{tabular}{l|l|l|l|l|l|l}
\hline \multirow{2}{*}{$\begin{array}{l}\text { Policy } \\
\text { Rule }\end{array}$} & \multicolumn{5}{|c|}{ Optimized reaction coefficients } & \multirow{2}{*}{ Welfare Loss } \\
\cline { 2 - 6 } & $\phi_{w}$ & $\phi_{\pi}$ & $\phi_{x}$ & $\phi_{q}$ & $\phi_{\psi}$ & \\
\hline 1 (C) & 1.8950 & 5.0801 & -0.0730 & 0.2825 & -0.2127 & 0.1719 \\
\hline 2 (EX) & 3.1280 & 11.2058 & 0.2115 & -0.1538 & 0 & 0.1724 \\
\hline 3 (PT) & 2.7212 & 9.4060 & 0.1144 & 0 & -0.1065 & 0.1722 \\
\hline 4 (TR) & 3.1698 & 10.8912 & 0.1169 & 0 & 0 & 0.1727 \\
\hline 5 (IT) & 3.2723 & 11.5622 & 0 & 0 & 0 & 0.1729 \\
\hline
\end{tabular}

A second general finding in Chapter 6 was that optimal simple policy rules that incorporate some smoothing (inertial policy rules) tend to work better than do optimal policy rules without smoothing (non-inertial policy rules). It is then of interest to examine whether this result still holds when private agents observe aggregate price with error. We begin by investigating the case where the reaction coefficient on the lagged interest is set at 0.8 . In this case the general policy form is written as

$$
\hat{R}_{t}=\phi_{w} \hat{r}_{H, t}^{f}+\phi_{\pi} \hat{\Pi}_{H, t}+\phi_{x} x_{t}+\phi_{q} q_{g, t}+\phi_{\Psi} \hat{\Psi}_{t}+0.8 \hat{R}_{t-1}
$$

These simulation results are presented in Table 7.3. When compared with those in 7.1 , we can see that welfare losses reported in Table 7.3 are significantly smaller than those arising in Table 7.1. The smallest welfare loss when the lagged interest rate term was not included in the policy rule is 0.1721 (see row 1 of Table 7.1 ), whereas it falls to 0.1600 in the first row of Table 7.3. This then reinforces our Chapter 6 finding that 
adding the lagged interest rate term into simple policy rules improves policy performance. Moreover, the less accurate is the information available to individuals, the greater will be the improvement in policy performance that can be made by adding the lagged interest rate term to the optimal simple policy rule. This is illustrated in Figure 7.1 where the percentage improvement in welfare from adding interest rate smoothing is shown across policy rules. Label I illustrates the percentage gain in the full information case, where for each rule the percentage gain is calculated as follows, the welfare loss associated each rule reported in Table 7.4 is subtracted that reported in Table 7.2 and then divided by the value in Table 7,4 . The same method is used to calculate values denoted by labels II and III, where label II represents the case in which private agents observe aggregate prices with error as reported in Tables 7.3 versus 7.1. Because the measurement error is relatively small (so that the actual improvement in the Tables is not easily visible in Figure 1) we use label III to denote the outcomes when a higher level of noise is adopted (as will be reported later in Tables 7.8 and 7.7). This better illustrate how increasing the noise in the problem increases the gain in policy performance that can be made with interest rate smoothing.

\section{Table 7.3}

Partially optimal inertial policy rules when inflation contains noise

\begin{tabular}{l|l|l|l|l|l|l}
\hline \multirow{2}{*}{$\begin{array}{l}\text { Policy } \\
\text { Rule }\end{array}$} & \multicolumn{5}{|c|}{ Optimized reaction coefficients } & Welfare Loss \\
\cline { 2 - 7 } & $\phi_{w}$ & $\phi_{\pi}$ & $\phi_{x}$ & $\phi_{q}$ & $\phi_{\psi}$ & \\
\hline $\mathbf{1 ~ ( C )}$ & 0.4449 & 1.1525 & -0.0308 & 0.1017 & -0.0539 & 0.1600 \\
\hline 2 (EX) & 0.4926 & 1.3375 & 0.0205 & 0.0046 & 0 & 0.1610 \\
\hline 3 (PT) & 0.4867 & 1.3579 & 0.0228 & 0 & 0.0037 & 0.1610 \\
\hline $4($ TR) & 0.4951 & 1.3486 & 0.0229 & 0 & 0 & 0.1610 \\
\hline $5($ IT) & 0.5282 & 1.4855 & 0 & 0 & 0 & 0.1612 \\
\hline
\end{tabular}


Table 7.4

Partially optimal inertial policy rules when information is complete

\begin{tabular}{l|l|l|l|l|l|l}
\hline \multirow{2}{*}{$\begin{array}{l}\text { Policy } \\
\text { Rule }\end{array}$} & \multicolumn{5}{|c|}{ Optimized reaction coefficients } & Welfare Loss \\
\cline { 2 - 7 } & $\phi_{w}$ & $\phi_{\pi}$ & $\phi_{x}$ & $\phi_{q}$ & $\phi_{\psi}$ & \\
\hline $1(\mathrm{C})$ & 0.4439 & 1.1482 & -0.0311 & 0.1023 & -0.0542 & 0.1598 \\
\hline 2 (EX) & 0.4920 & 1.3348 & 0.0204 & 0.0046 & 0 & 0.1608 \\
\hline $3(\mathbf{P T})$ & 0.4860 & 1.3553 & 0.0228 & 0 & -0.0037 & 0.1608 \\
\hline $4(\mathrm{TR})$ & 0.4903 & 1.3460 & 0.0229 & 0 & 0 & 0.1608 \\
\hline $5($ IT) & 0.5276 & 1.4830 & 0 & 0 & 0 & 0.1610 \\
\hline
\end{tabular}

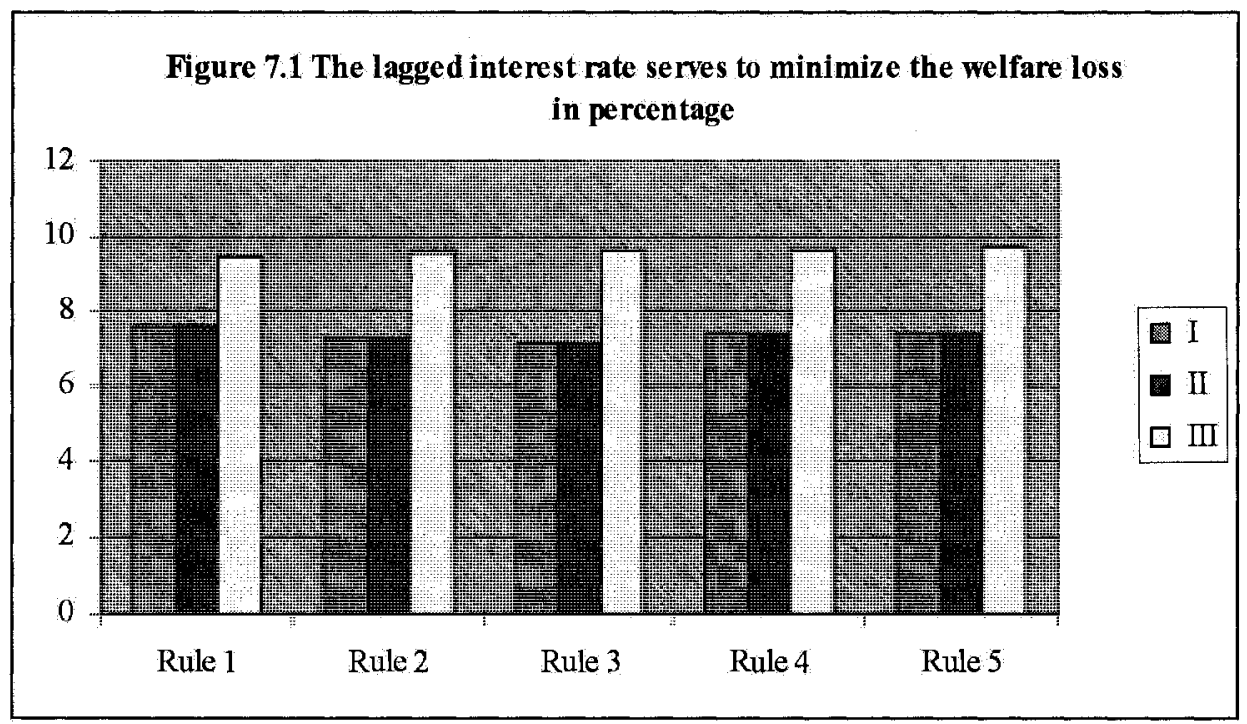

Two other findings deserve mention. First, note that the ranking of the optimal simple policy rules is retained under smoothing. Again the comprehensive rule works best while inflation targeting works least well. Second, the size of the reaction coefficient on the Wicksellian natural rate, $\phi_{w}$, gets smaller when the lagged interest rate is formally taken into account when adjusting the current period interest rate. For example, the coefficients of the Wicksellian natural rate are all smaller than 0.5 in Table 
7.3, while they are greater than 1.8 in Table 7.1. This is consistent with the findings observed in Chapter 6 and suggests that to some extent the lagged interest rate is a substitute for the Wicksellian rate in the policy rule.

We turn next to examine fully inertial optimal simple policy rules. In this case the reaction coefficient on the lagged interest rate is determined optimally rather than simply set by the policy maker. The general policy form for this case is

$$
\hat{R}_{t}=\phi_{w} \hat{r}_{H, t}^{f}+\phi_{\pi} \hat{\Pi}_{H, t}+\phi_{x} x_{t}+\phi_{q} q_{g, t}+\phi_{\Psi} \hat{\Psi}_{t}+\phi_{r} \hat{R}_{t-1}
$$

The simulation results for this case are showed in Table 7.5. Scanning it we see once again that the first three policy rules that incorporate the exchange rate in some form result in smaller welfare losses, with Rules 1 through 3 working better than Rules 4 and 5. In the complete private information analysis of Chapter 6 , we found that the coefficient of the lagged interest rate is greater than 1 when optimally determined and this observation is repeated here. When we compare the performances of two sets of inertial policy rules (see the welfare losses listed in Tables 7.3 and 7.5), we find, as expected, that the fully optimal simple policy rules work better than do their partially optimal alternatives. But, as also found in Chapter 6, the gain from incorporating a fully optimized, rather than an arbitrarily set, reaction coefficient on the lagged interest rate is smaller than the original gain involved from incorporating some form of the lagged interest rate term itself. Finally when we compare the performances of two sets of fully optimal policy rules with different information structures, we find the gain from fully optimal rules is higher under perfect information than with noise (see the welfare losses listed in Tables 7.5 and 7.6). The gain in performance from fully optimal smoothing rules fall as information becomes more incomplete. 


\section{Table 7.5}

Fully optimal inertial policy rules when inflation contains noise

\begin{tabular}{|c|c|c|c|c|c|c|c|}
\hline Policy & \multicolumn{6}{|c|}{ Optimized reaction coefficients } & \multirow{2}{*}{$\begin{array}{l}\text { Welfare } \\
\text { Loss }\end{array}$} \\
\hline Rule & $\phi_{w}$ & $\phi_{\pi}$ & $\phi_{x}$ & $\phi_{q}$ & $\phi_{\psi}$ & $\phi_{r}$ & \\
\hline 1 (C) & -0.0546 & 2.1007 & 0.0105 & 0.0039 & \begin{tabular}{|l|l|}
0.0298 \\
\end{tabular} & 1.9245 & 0.1588 \\
\hline $2(\mathbf{E X})$ & 0.0720 & 1.9391 & -0.0077 & 0.0390 & 0 & 1.6626 & \begin{tabular}{|l|l|}
0.15888 \\
\end{tabular} \\
\hline 3 (PT) & -0.0637 & 2.1146 & 0.0125 & 0 & 0.0322 & 1.9428 & 0.1588 \\
\hline 4 (TR) & 0.0687 & 2,0767 & 0.0114 & 0 & 0 & \begin{tabular}{|l|l|}
1.6597 \\
\end{tabular} & 0.1590 \\
\hline 5 (IT) & 0.0607 & 2.1955 & 0 & 0 & 0 & 1.7163 & 0.1591 \\
\hline
\end{tabular}

Table 7.6

Fully optimal inertial policy rules when information is complete

\begin{tabular}{l|l|l|l|l|l|l|l}
\hline \multirow{2}{*}{$\begin{array}{l}\text { Policy } \\
\text { Rule }\end{array}$} & \multicolumn{6}{|c|}{ Optimized reaction coefficients } & Welfare \\
\cline { 2 - 7 } & $\phi_{w}$ & $\phi_{\pi}$ & $\phi_{x}$ & $\phi_{q}$ & $\phi_{\psi}$ & $\phi_{r}$ & Loss \\
\hline 1 (C) & -0.0570 & 2.1011 & 0.0107 & 0.0036 & 0.0302 & 1.9290 & 0.1586 \\
\hline 2 (EX) & 0.0725 & 1.9345 & -0.0077 & 0.0392 & 0 & 1.6606 & 0.1587 \\
\hline 3 (PT) & -0.0657 & 2.1143 & 0.0125 & 0 & 0.0324 & 1.9464 & 0.1586 \\
\hline 4 (TR) & 0.0691 & 2.0736 & 0.0115 & 0 & 0 & 1.6583 & 0.1589 \\
\hline 5 (IT) & 0.0609 & 2.1932 & 0 & 0 & 0 & 1.7155 & 0.1589 \\
\hline
\end{tabular}

As mentioned in introduction and discussed briefly in relation to case 1, Svensson and Woodford (2003) hypothesize that the optimal response to imperfectly observed targeting variables will be inversely related to the degree of noise in those observations. In the limit, an extremely noisy measure will be ignored completely in the optimal policy rule. ${ }^{103}$ It is then of interest to examine whether their hypothesis is confirmed under our measurement error information structure (where private agents observe prices with error

${ }^{103}$ See Svensson and Woodford (2003), pp. 691-720. 
while policymakers observe the resulting prices accurately). As we have seen from comparing Table 7.1 with Table 7.2 , the optimized reaction coefficients across all optimal simple policy rules are typically smaller than those presented in Table 7.2 , especially the coefficients on inflation (our variable with error). For example, in Table 7.1 the coefficients of inflation and the Wicksellian natural rate under Rule 1 are 5.0377 and 1.8517 , respectively, while they are 5.0811 and 1.8950 in Table 7.2 . Similarly these two coefficients associated with Rule 2 in Table 1 are 10.4648 and 2.9829, respectively, while they are 11.2058 and 3.1280 in Table 7.2. In this case the results are consistent with the Svensson and Woodford conjecture.

What is interesting, however, is this observation is not repeated when we compare Tables 7.3 and 7.4, nor is it repeated in Tables 7.5 and 7.6. In neither of these cases does the coefficient size fall with an increase in the noise in the variable. Hence to further test whether the amount of noise contained in inflation has any effect on the coefficient size, we increase the degree of noise contained in the measure of inflation and redo Tables 7.1, 7.3 and 7.5, and report the results in Tables 7.7, 7.8, and 7.9, respectively. Comparing results in relevant Tables, we find that the same pattern of observations still holds. For example when we enlarge $\sigma_{p t}{ }^{2}$ from $0.005^{2}$ to $0.05^{2}$, the coefficients of inflation become much smaller under both non-inertial policy rules. The values of coefficient of inflation are all smaller than 5 with the maximum value 4.9 in Table 7.7 , while the coefficients are all greater than 5 with the maximum value 10.9 in Table 7.1. On the other hand, the values of coefficients of inflation in Table 7.8 are all larger than those reported in Table 7.3 and the same ranking is maintained when we compare the values of coefficient of inflation reported in Table 7.9 as compared to Table 7.5. 
Based on these findings we reach the following conclusion: the effect of a loss in private information on the size of the corresponding optimal coefficient in the policy response function will be ambiguous. In particular, it is not the case in our structure that the less perfect is private agents' knowledge of actual inflation, the lower will be the weight placed on inflation in the monetary authority's optimal response function.

\section{Table 7.7}

Non-Inertial optimal policy rules when inflation is observed with larger noise

\begin{tabular}{l|l|l|l|l|l|l}
\hline \multirow{2}{*}{$\begin{array}{l}\text { Policy } \\
\text { Rule }\end{array}$} & \multicolumn{5}{|c|}{ Optimized reaction coefficients } & Welfare Loss \\
\cline { 2 - 6 } & $\phi_{w}$ & $\phi_{\pi}$ & $\phi_{x}$ & $\phi_{q}$ & $\phi_{\psi}$ & \\
\hline 1 (C) & 1.9095 & 4.8287 & -0.0433 & 0.2221 & -0.1235 & 0.1931 \\
\hline 2 (EX) & 1.9572 & 4.9809 & 0.0712 & 0.0217 & 0 & 0.1935 \\
\hline 3 (PT) & 1.9002 & 4.8245 & 0.0848 & 0 & -0.0848 & 0.1936 \\
\hline 4 (TR) & 1.9164 & 4.8677 & 0.0845 & 0 & 0 & 0.1936 \\
\hline 5 (IT) & 1.9289 & 4.9749 & 0 & 0 & 0 & 0.1939 \\
\hline
\end{tabular}

Table 7.8

Partially optimal inertial policy rules when inflation contains more noise

\begin{tabular}{l|l|l|l|l|l|l}
\hline \multirow{2}{*}{$\begin{array}{l}\text { Policy } \\
\text { Rule }\end{array}$} & \multicolumn{5}{|c|}{ Optimized reaction coefficients } & Welfare Loss \\
\cline { 2 - 6 } & $\phi_{w}$ & $\phi_{\pi}$ & $\phi_{x}$ & $\phi_{q}$ & $\phi_{\psi}$ & \\
\hline $\mathbf{1}(\mathbf{C})$ & 0.5470 & 1.5660 & -0.0012 & 0.0419 & -0.0237 & 0.1764 \\
\hline $\mathbf{2}$ (EX) & 0.5558 & 1.5992 & 0.0211 & 0.0003 & 0 & 0.1766 \\
\hline $\mathbf{3}$ (PT) & 0.5519 & 1.6047 & 0.0212 & 0 & -0.0030 & 0.1766 \\
\hline 4 (TR) & 0.5557 & 1.5996 & 0.0212 & 0 & 0 & 0.1766 \\
\hline 5 (IT) & 0.5883 & 1.7198 & 0 & 0 & 0 & 0.1767 \\
\hline
\end{tabular}

Table 7.9

Fully optimal inertial policy rules when inflation contains more noise 


\begin{tabular}{|c|c|c|c|c|c|c|c|}
\hline Policy & \multicolumn{6}{|c|}{ Optimized reaction coefficients } & \multirow{2}{*}{$\begin{array}{l}\text { Welfare } \\
\text { Loss }\end{array}$} \\
\hline Rule & $\phi_{w}$ & $\phi_{\pi}$ & $\phi_{x}$ & $\phi_{q}$ & $\phi_{\psi}$ & $\phi_{r}$ & \\
\hline $1(\mathrm{C})$ & 0.0351 & 2.2428 & 0.0049 & 0.0096 & 0.0143 & 1.7932 & 0.1734 \\
\hline $2(\mathbf{E X})$ & 0.0533 & 2.2598 & -0.0057 & 0.0283 & 0 & 1.7651 & 0.1735 \\
\hline 3 (PT) & 0.0325 & 2.2399 & 0.0102 & 0 & 0.0187 & 1.7932 & 0.1734 \\
\hline 4 (TR) & 0.0590 & 2.2897 & 0.0090 & 0 & 0 & 1.7296 & 0.1736 \\
\hline 5 (IT) & 0.0604 & 2.3597 & 0 & 0 & 0 & 1.7544 & 0.1736 \\
\hline
\end{tabular}

Before we proceed to the case where both private and public agents encounter the same measurement error, let us summarize what we have found for the asymmetric case. First and foremost, our perfect private information conclusion that in a small open economy with price stickiness, incomplete exchange rate pass through and incomplete asset markets, the incorporation of some external measure into the policy rule -- the exchange rate and/or the law of one price gap - will improve policy performance can be extended to the case of private measurement error. Orphanides and Williams (2005b) claim that faced with structural change policies that appear to be optimal under perfect knowledge can perform very poorly if implemented in an environment of imperfect knowledge. This conjecture is not confirmed in our framework where it is transitory shocks that introduce imperfect information into the problem. ${ }^{104}$ The ranking of optimal simple policy rules under complete information remained unchanged when measurement asymmetries arose between private and public agents. This is our second observation. The third important observation is that inertial optimal simple policy rules again perform better than non-inertial policy rules and fully optimal simple policy rules continue to perform better than do partially optimal simple policy rules. As expected, social welfare

${ }^{104}$ We can report here that simulations done for the case where measurement error incorporated persistence increased the size of the welfare loss but did not change the ordering across money rules. Hence our findings for the case of i.i.d. measurement errors generalize at least in this dimension. 
is lower the more inaccurate is the aggregate price information held by private agents with social welfare loss positively related to the degree of noise contained in the observed rate of inflation. Finally, we cannot confirm for our case the Svensson and Woodford (2003) conjecture that the weight that should be placed on inflation in the policy rule should vary inversely with the accuracy of private agent's information on that variable.

\subsection{Optimal Simple Policy Rules when both Private Agents and Policymakers Face} the Same Measurement Error

While policymakers such as the central bank have some advantages over private agents in information collecting and processing, it is not unreasonable to assume that policymakers often face similar measurement issues when making policy. Here for convenience we consider the case where all agents observe current aggregate prices with a common error. Other indicators and historical prices can be observed accurately. Thus our information structure is symmetric and incomplete. Finally, to make the results comparable across with the asymmetric case above, we assume that the aggregate price levels observed by the central bank are defined as did in section 7.2.

To investigate the effects of symmetric incomplete information on optimal simple policy rules and associated welfare losses we follow the preceding section and first examine non-interest rate (inertial) optimal simple policy rules, and then move on to (inertial) policy rules with interest rate smoothing. The general form of non-inertial policy can be expressed as

$$
\hat{R}_{t}=\phi_{w} \hat{r}_{H, t}^{f}+\phi_{\pi}\left(\hat{\Pi}_{H, t}\right)^{0}+\phi_{x} x_{t}+\phi_{q} q_{g, t}+\phi_{\Psi} \hat{\Psi}_{t}
$$


where $\left(\hat{\Pi}_{H, t}\right)^{0}$ denotes the deviation of inflation from steady state directly observed by policymakers.

The simulation results for our five policy rules are presented in Table 7.10. Looking at the welfare loss, we see that Rule 1 works best. The welfare loss arising under its use is 0.1729 . Performing second and third best are Rules 3 and 2; with the associated welfare losses arising under their use being 0.1746 and 0.1750 , respectively. ${ }^{105}$ The Taylor rule and the Inflation Targeting rule result in the largest welfare losses, and therefore they are ranked at the bottom. This performance pattern across policy rules is once again exactly the same as that found in Chapter 6 and the previous section, which implies that the scale of measurement noise has not affected the relative performance of alternative policies. Comparing the welfare losses reported in Table 7.10 with those reported in Table 7.1, however, we do see that the level of performance that can be expected from policy has been affected by noise. For example, the smallest welfare loss in Table 7.10 is 0.1729 , which is larger than its counterpart in Table $7.1(0.1721)$. This leads us to the following conclusion: the accurateness of the information received by agents does not alter the relative ranking of alternative policy rules, but does worsen the overall outcome.

Table 7.10

Non-inertial optimal policy rules when information is symmetric

\begin{tabular}{l|l|l|l|l|l|l}
\hline \multirow{2}{*}{$\begin{array}{l}\text { Policy } \\
\text { Rule }\end{array}$} & \multicolumn{5}{|c|}{ Optimized reaction coefficients } & Welfare Loss \\
\cline { 2 - 6 } & $\phi_{w}$ & $\phi_{\pi}$ & $\phi_{x}$ & $\phi_{q}$ & $\phi_{\psi}$ & \\
\hline $1(\mathrm{C})$ & 1.2198 & 1.9326 & -0.1342 & 0.3801 & -0.2284 & 0.1729 \\
\hline
\end{tabular}

${ }^{105}$ Note that the differences in welfare across rules and across cases stand out more strongly as the size of the information problem becomes bigger. This is a further example of what was demonstrated in Tables 7.8 and 7.9 . 


\begin{tabular}{l|l|l|l|l|l|l}
\hline 2 (EX) & 1.8418 & 4.8629 & 0.1314 & -0.0573 & 0 & 0.1750 \\
\hline 3 (PT) & 1.7471 & 4.5358 & 0.0995 & 0 & -0.0510 & 0.1746 \\
\hline 4 (TR) & 1.9132 & 5.0017 & 0.0949 & 0 & 0 & 0.1752 \\
\hline 5 (IT) & 1.9307 & 5.1543 & 0 & 0 & 0 & 0.1757 \\
\hline
\end{tabular}

Following our previous structure, we next investigate the performance of adding partial inertia to the previous optimal simple policy rule without smoothing. Such a partially inertial optimal simple policy rule can be expressed as

$$
\hat{R}_{t}=\phi_{w} \hat{r}_{H, t}^{f}+\phi_{\pi}\left(\hat{\Pi}_{H, t}\right)^{0}+\phi_{x} x_{t}+\phi_{q} q_{g, t}+\phi_{\Psi} \hat{\Psi}_{t}+0.8 \hat{R}_{t-1} .
$$

Equations (7.31) is exactly the same as equation (7.28), except that the actual inflation, $\hat{\Pi}_{H, t}$ in equation (7.28) is replaced by the rate of inflation actually observed $\left(\hat{\Pi}_{H, t}\right)^{0}$. Scanning the results presented in Table 7.11 , we see that all key findings extracted from Table 7.10 remain (the welfare losses across rules being smaller than those presented in Table 7.10). Hence once again the results show that inertial policy rules perform better than non-inertial policy rules. Comparing the welfare losses in Table 7.11 with those reported in Table 7.3, we see that even with partial interest rate smoothing the inability to observe aggregate price levels accurately by both individuals and the central bank does increase the size of welfare losses.

Table 7.11

Partially inertial optimal policy rules when measurement error is symmetric

\begin{tabular}{l|l|l|l|l|l|l}
\hline \multirow{2}{*}{$\begin{array}{l}\text { Policy } \\
\text { Rule }\end{array}$} & \multicolumn{5}{|c|}{ Optimized reaction coefficients } & Welfare Loss \\
\cline { 2 - 7 } & $\phi_{\psi}$ & $\phi_{\pi}$ & $\phi_{x}$ & $\phi_{q}$ & $\phi_{\psi}$ & \\
\hline $\mathbf{1}(\mathbf{C})$ & 0.4257 & 1.0773 & -0.0288 & 0.1039 & -0.0549 & 0.1606 \\
\hline $2 \mathbf{( E X )}$ & 0.4679 & 1.2412 & 0.0245 & 0.0044 & 0 & 0.1617 \\
\hline $3 \mathbf{( P T )}$ & 0.4617 & 1.2601 & 0.0268 & 0 & -0.0037 & 0.1617 \\
\hline
\end{tabular}




\begin{tabular}{l|l|l|l|l|l|l}
\hline 4 (TR) & 0.4659 & 1.2509 & 0.0269 & 0 & 0 & 0.1617 \\
\hline 5 (IT) & 0.5064 & 1.3971 & 0 & 0 & 0 & 0.1620 \\
\hline
\end{tabular}

Finally, a fully optimal inertial simple policy rule for the central bank that observes aggregate prices with error can be written as

$$
\hat{R}_{t}=\phi_{w} \hat{r}_{H, t}^{f}+\phi_{\pi}\left(\hat{\Pi}_{H, t}\right)^{0}+\phi_{x} x_{t}+\phi_{q} q_{g, t}+\phi_{\Psi} \hat{\Psi}_{t}+\phi_{r} \hat{R}_{t-1}
$$

As expected, the simulation results summarized in Table 7.12 indicate that welfare losses can be decreased further by optimizing over the lagged interest rate. This can be seen by scanning the results in Table 7.12 relative to those in Table 7.11). Hence in a more clearly observable way, our results confirm the hypothesis that fully optimal inertial policy rules work better than do arbitrary inertial rules.

Table 7.12

Fully optimal inertial policy rules when measurement error is symmetric

\begin{tabular}{l|l|l|l|l|l|l|l}
\hline \multirow{2}{*}{$\begin{array}{l}\text { Policy } \\
\text { Rule }\end{array}$} & \multicolumn{6}{|c|}{ Optimized reaction coefficients } & \multirow{2}{*}{ Welfare } \\
\cline { 2 - 7 } & $\phi_{w}$ & $\phi_{\pi}$ & $\phi_{x}$ & $\phi_{q}$ & $\phi_{\psi}$ & $\phi_{r}$ & Loss \\
\hline 1 (C) & 0.2730 & 1.4312 & -0.0103 & 0.0604 & -0.0221 & 1.1707 & 0.1602 \\
\hline $2(\mathbf{E X )}$ & 0.2072 & 1.5546 & 0.0054 & 0.0301 & 0 & 1.3227 & 0.1603 \\
\hline $3($ PT) & 0.1809 & 1.6366 & 0.0212 & 0 & 0.0132 & 1.3800 & 0.1604 \\
\hline 4 (TR) & 0.2252 & 1.6196 & 0.0208 & 0 & 0 & 1.2764 & 0.1605 \\
\hline $5($ IT) & 0.2321 & 1.7798 & 0 & 0 & 0 & 1.3296 & 0.1607 \\
\hline
\end{tabular}

In the previous section where private agents alone observed aggregate price levels with error, there was not an unequivocal answer to whether the value of optimized reaction coefficient on inflation in the policy rule should become smaller as the noise contained in inflation becomes larger. It is then of interest to know if this remains true when the same measurement issue also faces policymakers. Comparing the size of the 
optimized reaction coefficients, we can see that the coefficient values on inflation across rules appearing in Tables 7.10 though 7.12 are all smaller than the corresponding ones reported in Tables 7.1, 7.3 and 7.5. Hence our findings are consistent with Svensson and Woodford. To illustrate the differences made across our different measurement cases, we use the following three diagrams. In Figure 7.2 we present the three optimal inflation reaction coefficients for the five policy non-interest rate smoothing rules that correspond to the cases of no measurement error (Table 7,2 results labeled as A), private measurement error (Table 7.1 results labeled as B) and private and public symmetric measurement error (Table 7.10 results labeled as $\mathrm{C}$ ). Figure 7.3 repeats the same basic structure for partial interest rate smoothing (from Tables 7.4, 7.3 and 7.11). Finally in Figure 7.4 we present the three coefficient values for the five different policy rules utilizing fully optimal interest rate smoothing (from Tables 7.6, 7.5, and 7.12). Again for the three coefficients; Label A represents the perfect information case in which all agents are able to access to accurate complete information; Label B denotes the case of asymmetric private measurement error modeled in section 7.3; and Label $\mathrm{C}$ represents the case where measurement error is symmetric for both private agents and the monetary authority.

From Figure 7.2 , we can see that for each of the five policy rules, the inflation coefficient in the optimal policy rule labeled B is smaller than for label $A{ }^{106}$ But the same observation does not hold in Figures 7.3 and 7.4. In these figures, the weight placed on inflation under label B are very close to that under label A but are often larger. However, the picture is quite different when we compare the values represented by label

\footnotetext{
${ }^{106}$ This is true for Rule 1 but not apparent on the diagram because of the relatively small size of the measured difference.
} 
$\mathrm{C}$ (the case in which both private agents and policymakers face measurement error issue) with those labeled A (no measurement error issue). The weights placed on inflation across rules under label $\mathrm{C}$ in Figure 7.2 fall dramatically less than half their value under label A. In Figures 7.3 and 7.4, the fall is not as dramatic, but all inflation coefficients are now smaller in value than those represented by label A. To the extent we compare fully optimal rules, the Svensson and Woodford hypothesis is confirmed by our analysis.
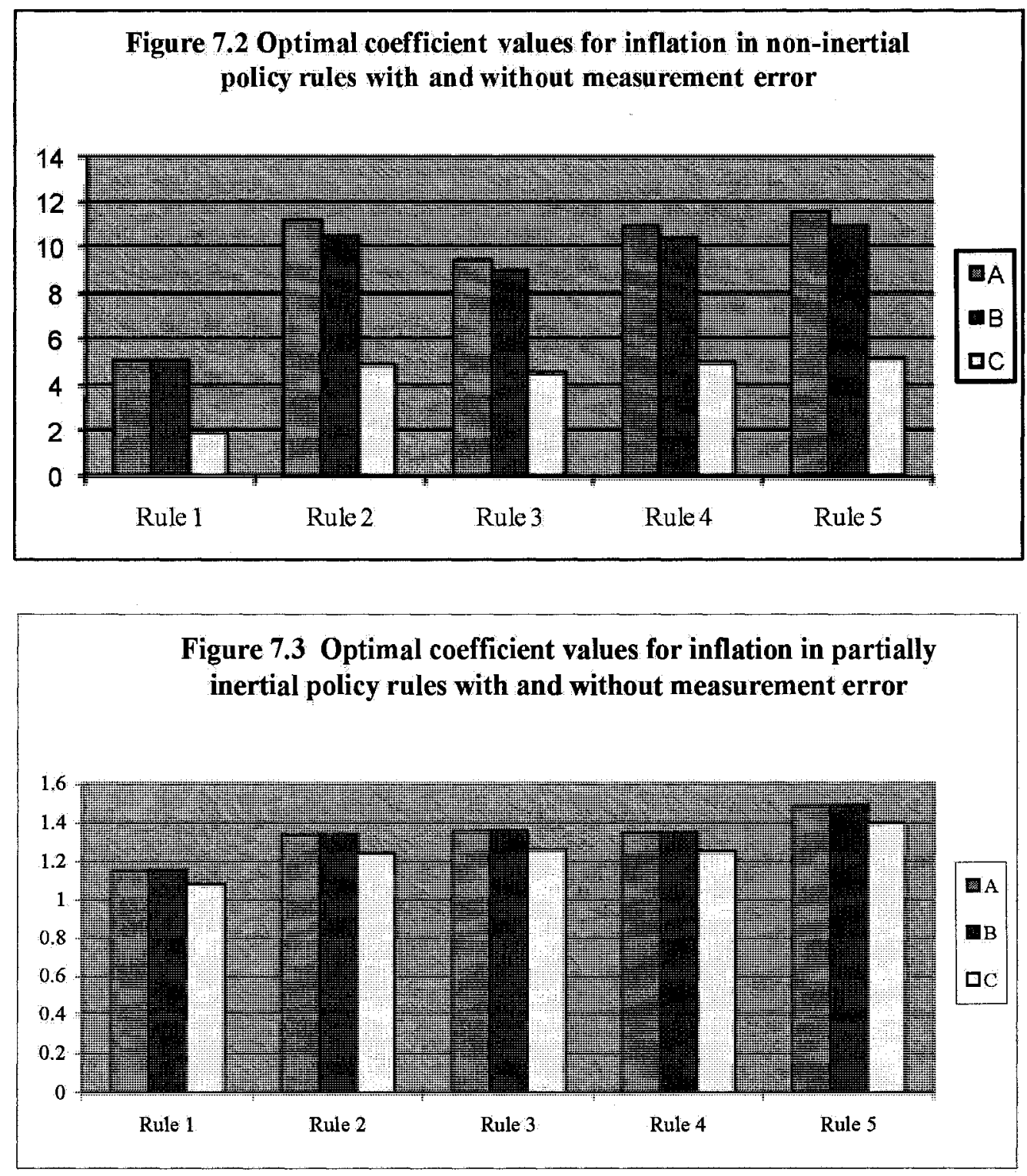


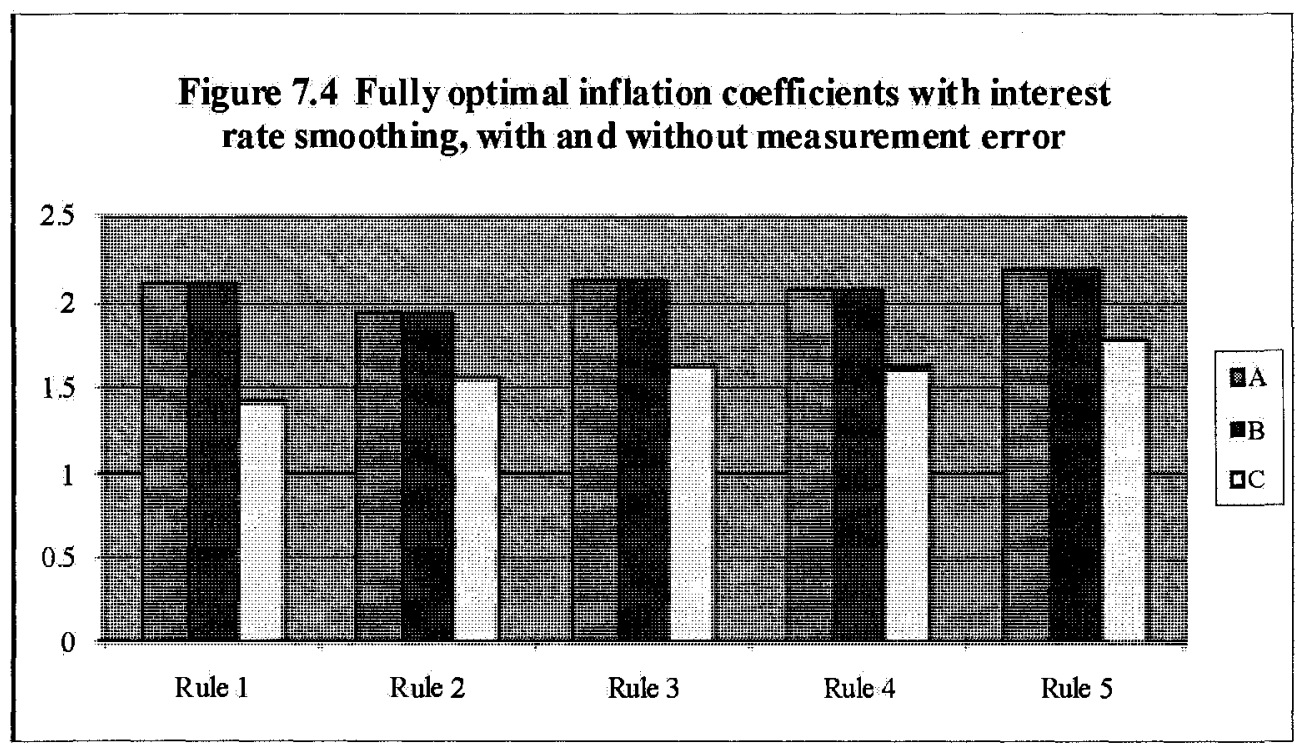

We have shown that optimal central bank behave is to respond more cautiously in the money rule to variables that are more noisy. When the noisy variable is inflation, the optimal response is to weigh inflation less in the policy rule. The next question to be asked is whether the central bank should react to other variables in the money rule in the same manner. For example should the Wicksellian natural rate and/or the output gap be treated more cautiously as well or, in contrast, should the weights be larger and monetary policy behave more aggressively with respect to these other indicators? Svensson and Woodford (2003) do not raise this issue explicitly, but from the analytical solution to their optimal policy rule, we see that the coefficients of the other indicators are independent of measurement noise and therefore will be unaffected by its size or even presence.

However, the simulation results above show that with measurement error, the optimized values of the coefficients of the other variables in the policy rule are affected as well. This is illustrated for the coefficient of the Wicksellian natural rate in Figure 7.5 (see Tables7.2, 7.1, and 7.10). As can be seen there, the optimal weights put on the 
Wicksellian natural rate when both individuals and policy makers observe prices with measurement error (label C) are much smaller those arising when only individuals experience measurement error (label B) or neither experience measurement error (label A). A similar picture could be drawn for the lagged interest rate. It should be mentioned that optimal weights of other variables are not always decreased, under some cases they increase a bit. For example, the optimal weight to be put on the output gap under Rule 4 in the symmetric case is 0.0208 (see Table 7.12), while being only 0.0115 in the perfect information case (see Table 7.6).

The reason why the weights on the other variables are affected can be attributed to the forward-looking feature of our model and the endogenous nature of the targeted variables. In a forward-looking model, current period endogenous non-predetermined variables, such as inflation and the output gap depend on private-sector expectations of present and future endogenous variables and present and future policy responses. Hence when any one endogenous variable contains noise, that noise will be transmitted to other variables via private-sector expectations and market interactions. With more mistakes (relative to perfect information), more noise is transmitted and hence it becomes optimal for policymakers to act cautiously to these variables as well, Nevertheless, because all variables will not be contaminated by noise proportionally, some change in the relative weighting under optimization can be expected. 


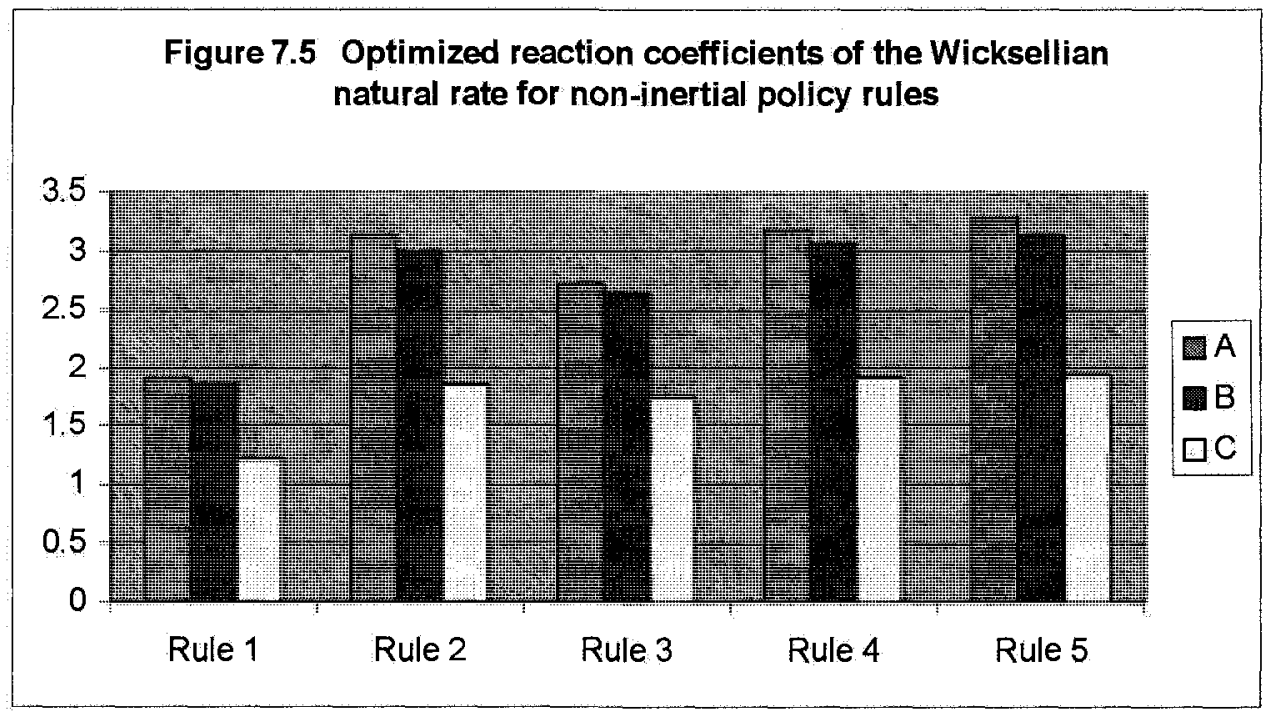

\subsection{Concluding remarks}

The problem of how decision making should respond to the lack of fully accurate information is an important issue that continues to confront policymakers. In previous chapters we explored this issue at length for cases where policymakers were denied successively more information while private agents were assumed to remain fully informed. For this to translate into an important contribution to policy, the results would need to remain qualitatively unchanged when private agents possess less than complete information. In this chapter we explore this proposition in greater depth by assuming first that private agents can observe the relevant aggregate price levels only with error. From this analysis we can derive our first important insight, that for this case of private measurement error the relative ranking of optimal simple policy rules remains unchanged. Our conclusion that some recognition of the external side of the economy should be present in the money rule is more robust. A second dimension of the chapter 
has been to explore whether these findings persist when the measurement error is made symmetric for policy makers.

The main conclusions of this chapter are as follows. First, incomplete information in the sense of measurement error leads to welfare loss, regardless of whether that loss accrues to private agents or policymakers. The less accurate is the information available, the more welfare will suffer. In our framework the symmetric information structure matters primarily because it implies even less information available than in the asymmetric information case, and therefore results in a higher welfare loss. In relative terms, the fall in welfare tends to be larger when the measurement issue is experienced by the policy maker. Second, while information deficiency weakens policy effectiveness, it does not in our cases alter the relative efficiency of the alternative policy rules. In our analysis, the comprehensive policy rule is always at the top of the list, while the inflation targeting rule is always at the bottom. Thus Orphanides and Williams' (2005b) conjecture that policies that appear optimal under perfect knowledge perform poorly if implemented in an environment of imperfect knowledge is not supported by our work. Third, and closely related to the main contribute mentioned above is that under our assumptions policy rules incorporated exchange rate in some form perform better than or at least work as well as do the Taylor rule and the inflation targeting rule. As importantly, there is no change in relative ranking of optimal simple money rules whether the measurement error is experienced symmetrically or asymmetrically across private and public agents.

In terms of the coefficients within the policy rules, the optimal weight placed on the indicators that can be observed only with noise receives less weight in the policy rule 
than otherwise. An increase in noise strictly reduces policy weight. And lastly, in a dynamic forward-looking general equilibrium model all endogenous variables interact. Therefore as long as one variable contains noise, the noise in that variable will be transmitted to others via individual expectations and market interactions in general equilibrium. Hence the optimal weight assigned to these contaminated indicators will tend to fall, but because the degree of interaction depends upon the specific model, the relative weighting can decrease or increase depending upon specific circumstance. 


\section{References}

Adolfson, Malin, 2007, "Incomplete Exchange Rate Pass-Through and Simple Monetary Policy Rules," Journal of International Money and Finance, (26), pp. 468-494.

Amato, J., and T. Laubach, 1999, "The Value of Interest Rate Smoothing: How the Private Sector Helps the Federal Reserve." Economic Review, Federal Reserve Bank of Kansas City, 84, pp. 47-64.

Ambler, Steve, Ali Dib, and Nooman Rebei, 2003, "Nominal Rigidities and Exchange Rate Pass-Through in a Structural Model of a Small Open Economy", Bank of Canada, Working Paper: No.29.

Ambler, Steve, Ali Dib, and Nooman Rebei, 2004, "Optimal Taylor Rules in an Estimated Model of a Small Open Economy.” Bank of Canada, Working Paper: No.36.

Anderson, Brian D. O. and John B. Moore, 1979, Optimal Filtering, Prentice Hall, Inc., New Jersey,

Anderson, Brian D. O. and John B. Moore, 1990, Optimal Control: Linear Quadratic Methods, Prentice Hall, Inc., New Jersey.

Anderson, Gary and George Moore, 1985, "A Linear Algebraic Procedure for Solving Linear Perfect Foresight Models." Economics Letters, 17, pp. 247-252.

Aoki, Kosuke, 2001, "Optimal Policy Responses to Relative-price Changes," Journal of Monetary Economics, 48, pp. 58-50. 
Aoki, Kosuke, 2002, "Optimal Commitment Policy under Noisy Information." CREI, Universitat Pompeu Fabra and CEPR.

Aoki, Kosuke, 2003, "On the Optimal Monetary Policy Response to Noisy Indicators." Journal of Monetary Economics 50, pp. 501-523.

Attfield, C. L. F., D. Demery and N. W. Duck, 1991, Rational Expectations in Macroeconomics: An introduction to Theory and Evidence. Basil Blackwell, Inc.

Backus, D. and J. Driffill, 1986, "The Consistency of Optimal Policy in Stochastic Rational Expectations Models," Discussion Paper No. 124, Center for Economic Policy Research, London.

Barro, R. J. and D. B. Gordon, 1983, "Rules, Discretion, and Reputation in a Model of Monetary Policy", Journal of Monetary Economics, 12(1), pp. 101-121.

Batini, Nicoletta and Andrew G. Haldane, 1999, "Forwarding-Looking Rules for Monetary Policy," in John B. Taylor, ed., Monetary Policy Rules, Chicago: University of Chicago Press.

Batini, Nicoletta, Richard Harrison, and Stephen P, Millard, 2003, "Monetary Policy Rules for an Open Economy," Journal of Economic Dynamics and Control, 27, pp. 2059-2094.

Bellman, R., 1957, Dynamic Programming, Princeton, N. J., Princeton University Press. Benigno, P., 2001, "Price Stability with Imperfect Financial Integration," New York University, manuscript.

Benigno, Gianluca, and Pierpaolo Benigno, 2000, "Monetary Policy Rules and the Exchange Rate," Bank of England, manuscript. 
Benigno, Gianluca and Pierpaolo Benigno, 2003, "Price Stability in Open Economies", The Review of Economic Studies, vol. 70, pp. 743-765.

Benigno, Pierpaolo, 2004, "Optimal Monetary Policy in a Currency Area," Journal of International Economics, 63(2), pp. 293-320.

Bernanke, Ben S., Thomas Laubach, Frederic S. Mishkin, and Adam S. Posen, 1999, Inflation Targeting: Lessons from the International Experience, Princeton University Press, Princeton and Oxford.

Bertsekas, Dimitri P., 1976, Dynamic Programming and Stochastic Control. Academic Press, New York.

Betts, Caroline, and Michael B. Devereux, 1997, "The International Monetary Policy Transmission Mechanism: A Model of Real Exchange Rate Adjustment under Pricing to Market," University of British Columbia, manuscript.

Betts, Caroline, and Michael B. Devereux, 1998, "The International Effects of Monetary Policy in a Two-Country Model," University of British Columbia, manuscript.

Binder, Michael and M. Hashem Pesaran, 1995, "Multivariate Rational Expectations Models and Macroeconometric Modeling: A Review and Some New Results." In M. Hashem Pesaran and Mike Wickens, eds., Handbook of Applied Econometrics: Macroeconomics, Blackwell Publisher Ltd., Oxford, UK.

Binder, Michael and M. Hashem Pesaran, 1996, "Multivariate Linear Rational Expectations Models: Characterization of the Nature of the Solutions and Their Fully Recursive Computation," University of Maryland, manuscript.

Blanchard, Oliver, 2007, "Current Account Deficits in Rich Countries," IMF Staff Papers, 54(2), pp. 191-219. 
Blanchard, Oliver J. and Stanley Fischer, 1989, Lectures on Macroeconomics, The MIT Press, Cambridge.

Blanchard, Olivier Jean and Charles M. Kahn, 1980, "The solution of Linear Difference Models under Rational Expectations." Econometrica, 48, pp. 1305-1312.

Bowman, David and Brian Doyle, 2003, "New Keynesian, Open Economy Models and Their Implications for Monetary Policy," Board of Governors of the Federal Reserve System, International Finance Discussion Papers No. 762, March.

Bullard, James, and Kaushik Mitra, 2002, "Learning about Monetary Policy Rules." Journal of Monetary Economics, 49, pp. 1105-1129.

Calvo, G, A., 1978, "On the Time Consistency of Optimal Policy in a Monetary Economy," Econometrica, 46, pp. 1411-1428.

Campa, Jose Manuel, and Linda S. Goldberg, 2002, "Exchange Rate Pass-Through into Import Prices: A Macro or Micro Phenomenon?" Federal Reserve Bank of New York, mimeo.

Casares, M., 2001,"Business Cycle and Monetary Policy Analysis in a Structural Stickprice Model of the euro area". ECB Working Paper No. 49.

Chari, V.V., Patrick J. Kehoe, and Ellen R. McGrattan, 2002, "Can Stick Price Models Generate Volatile and Persistent Real Exchange Rates?” Research Department Staff Report 277, Federal Reserve Bank of Minneapolis.

Choudhri, Ehsan U., Hamid Faruque, and Dalia S. Hakura, 2005, "Explaining the Exchange Rate Pass-Through in Different Prices," Journal of International Economics, 65, pp. 349-374. 
Choudhri, Ehsan U., 2005, "Monetary Policy in a Small Emerging Economy: Exploring Desirable Interest Rate Rules," Carleton University, manuscript.

Chow, Gregory C., 1975, Analysis and Control of Dynamic Economic Systems. John Wiley \& Sons, Inc, New York.

Chow, Gregory C., 1997, Dynamic Economics. Oxford University Press, New York.

Christiano, Lawrence J, Martin Eichenbaum, and Charles Evans, 2001, Nominal Rigidities and the Dynamic Effects of a Shock to Monetary Policy, NBER working paper 8403 .

Clarida, Richard, Jordi Gali and Mark Gertler, 1998, "Monetary Policy Rules in Practice: Some International Evidence." European Economic Review, 42, pp. 1033-67.

Clarida, Richard, Jordi Gali, and Mark Gertler, 1999, "The Science of Monetary Policy: A New Keynesian Perspective," Journal of Economic Perspectives, 37(4), pp. $147-180$.

Clarida, Richard, Jordi Gali, and Mark Gertler, 2000, "Monetary Policy Rules and Macroeconomic Stability: Evidence and Some Theory," Quarterly Journal of Economics, 115(1), February, pp. 147-180.

Clarida, Richard, Jordi Gali, and Mark Gertler, 2001, “Optimal Monetary Policy in Open versus Closed Economies: An Integrated Approach," AEA Papers and Proceedings, Vol.91, No. 2, pp. 248-252.

Clarida, Richard, Jordi Gali, and Mark Gertler, 2002, "A Simple Framework for International Monetary Policy Analysis," Journal of Monetary Economics, 49, pp. 879-904. 
Clark, Peter B., Charles A. E. Goodhart and Haizhou Huang, 1999, "Optimal Monetary Policy Rules in a Rational Expectations Model of the Phillips Curve." Journal of Monetary Economics, 43, pp. 497-520.

Corsetti, S., and P Pesenti, 2001, "Welfare and Macroeconomic Interdependence," Quarterly Journal of Economics, 116(2), pp. 421-446.

Corsetti, Giancarlo, and Paolo Pesenti, 2005, "International Dimensions of Optimal Monetary Policy," Journal of Monetary Economics, 52(2), pp. 281-305.

Cukierman, Alex, Inflation, Stagflation, Relative Prices, and Imperfect Information, Cambridge University Press, Cambridge.

Currie, David and Paul Levine, 1982, "A Solution Technique for Discrete and Continuous Time Stochastic Dynamic Models under Rational Expectations with Full and Partial Information Sets," QMC PRISM paper No.1.

Currie, David and Paul Levine, 1987, "Credibility and Time Inconsistency in a Stochastic World," Journal of Economics, 47(3), pp. 225-252.

Currie, David and Paul Levine, 1993, Rules, Reputation and Macroeconomic Policy Coordination, Cambridge University Press, Cambridge.

De Paoli, Bianca, 2006, "Monetary Policy and Welfare in a Small Open Economy", CEP discussion paper NO 639.

Devereux, Michael B. and Charles Engel, 2003, "Monetary Policy in the Open Economy Revisited: Price Setting and Exchange-Rate Flexibility," Review of Economic Studies, Vol. 70, No. 4. pp. 765-783.

Dennis, Richard, 2004, "Solving for Optimal Simple Rules in Rational Expectations Models." Journal of Economic Dynamics \& Control, 28, pp. 1635-1660. 
Dennis, Richard, 2005, "Optimal Policy in Rational Expectations Models: New Solution Algorithms." Federal Reserve Bank of San Francisco, working paper 2005-09.

Dib, Ali, 2003, "Monetary Policy in Estimated Models of Small Open and Closed Economies," Bank of Canada, Working Paper No. 2003-27.

Domowitz, I., Hubbard, G., Petersen, B., 1988, "Market structure and cyclical fluctuations in U.S. Manufacturing”, Review of Economics and Statistics 80, pp. $55-66$.

Engel, Charles, 1993, "Real Exchange Rates and Relative Prices: An Emperical Investigation," Journal of Monetary Economics, 32, pp. 35-50.

Engel, Charles, 2002, "Expenditure Switching and Exchange Rate Policy," NBER Macroeconomics Annual, 17, pp. 231-272.

Erceg, Christopher, J., Dale W. Henderson, and Andrew T. Levin, 2000, “Optimal Monetary Policy with Staggered Wage and Price Contracts." Journal of Monetary Economics, 46, pp. 281-313.

Evans, George W. and Seppo Honkapohja, 2001, Learning and Expectations in Macroeconomics. Princeton University Press, Princeton and Oxford.

Fuhrer, Jeffrey C. and George R. Moore, 1995, "Inflation Persistence." Quarterly Journal of Economics, February, No. 440, pp. 127-159.

Gali, Jordi, 1999, "Technology, Employment, and the Business Cycle: Do Technology Shocks Explain Aggregate Fluctuations?" American Economic Review, 89 (1), pp. 249-271.

Gali, Jordi and Mark Gertler, 1999, "Inflation Dynamics: A Structural Econometric Analysis." Journal of Monetary Economics, Vol. 44, No. 2, pp. 195-222. 
Gali, Jordi, Mark Gertler, and D. Lopez-Salido, 2001, "European Inflation Dynamics." European Economic Review, 45 (7), pp. 1237-1270.

Gali, Jordi, and Tommaso Monacelli, 2002, "Optimal Monetary Policy and Exchange Rate Volatility in a Small Open Economy," NBER Working Paper, No. 8905.

Gali, Jordi and Tommaso Monacelli, 2005, "Monetary Policy and Exchange Rate Volatility in a Small Open Economy”, Review of Economic Studies, vol. 75, pp. 707-734.

Gavin, Willian T., Benjamin D. Keen, and Michael R. Pakko, 2005, "The Monetary Instrument Matters." Federal Reserve Bank of St. Louis Review, September/October, Volume 87, No. 5, pp. 633-658.

Ghironi, F., 1999, "Alternative Monetary Rules for a Small Open Economy: The Case of Canada," Federal Reserve Bank of New York, manuscript.

Goldberg, Pinelopi, and Michael Knetter, 1997, “Goods Prices and Exchange Rates: What We Have Learned?" Journal of Economic Literature, Vol. 35, pp. 1243-92.

Golub, G. H., Van Loan, C. F., 1983, Matrix Computations. The Johns Hopkins University Press, Blatimore, Maryland.

Goodfriend, Marvin, 1991, Interest Rate Smoothing in the Conduct of Monetary Policy," Carnegie-Rochester Conference Series on Public Policy, Spring, pp. 7-30.

Goodfriend, Marvin and Robert G. King, 1997, "The New Neoclassical Synthesis and the Role of Monetary Policy," NBER Macroeconomics Annual 1997, pp.231-283.

Harvey, Andrew C., 1989, Forecasting, Structural Time Series Models and the Kalman Filter. Cambridge University Press, Cambridge. 
Hooper, P. and J. Marquez, 1995, "Exchange Rates, Prices, and External Adjustment in the United States and Japan, in Kenen, P. ed., Understanding Interdependence, Princeton University Press, Princeton, pp. 107-168.

Huang, Kevin X. D., and Zheng Liu, 2005, "Inflation Targeting: What Inflation Rate to Target?" Journal of Monetary Economics, 52, pp. 1435-1462.

Ireland, Peter, 2001, "Sticky-Price Models of the Business Cycle: Specification and Stability," Journal of Monetary Economics, 47(1), pp. 3-18.

Ireland, Peter, 2004, "Technology Shock in the New Keynesian Model," NBER Working Paper, 10309.

Judd, Kenneth L., 1998, Numerical Methods in Economics. The MIT Press, Cambridge, Massachusetts.

Keynes, J. M., 1923, A Tract on Monetary Reform, London: Macmillan.

Kim, J., and S. Kim, 2003, "Spurious Welfare Reversals in International Business Cycle Models," Journal of International Economics, 60, pp. 471-500.

Kim, J., S. Kim, E. Schaumbeg, and C. A. Kim, 2003, "Calculating and Using Second Order Accurate Solutions of Discrete Time Dynamic Equilibrium Models," Princeton University, manuscript.

Kirsanova, Tatiana, Campbell Leith and Simon Wren-Lewis, 2006, "Should Central Banks Target Consumer Prices or the Exchange Rate?" The Economic Journal, 116 , pp. 208-231.

Klein, Paul, 2000, "Using the Generalized Schur Form to Solve a Multivariate Linear Rational Expectations Model." Journal of Economic Dynamic \& Control, 24, pp. 1405-1423. 
Kollmann, Robert, 1997, “The Exchange Rate in a Dynamic Optimizing Current Account Model with Nominal Rigidities," IMF, manuscript.

Kollmann, Robert, 1999, "Explaining International Co-movements of Output and Asset Returns: the Role of Money and Nominal Rigidities," IMF, manuscript.

Kollmann, Robert, 2002a, "Monetary Policy and Exchange Rate Volatility in a Small Open Economy," Review of Economic Studies, 49(5), pp. 989-1015.

Kollmann, R. 2002b, "Monetary Policy Rules in the Open Economy: Effects on Welfare and Business Cycles." Journal of Monetary Economics, 49, pp. 989-1015.

Kozocki, S., 1999, "How Useful Are Taylor Rules for Monetary Policy?" Economic Review, Federal Reserve Bank of San Francisco, pp. 3-16.

Kwakernaak, Huibert and Raphael Sivan, 1972, Linear Optimal Control Systems. John Wiley \& Sons, Inc., New York.

Kydland, F. E. and E. C. Prescott, 1982, "Time to Build and Aggregate Fluctuation," Econometrica, 6, pp. 1345-1370.

Lane, Philip R., 2001, “The New Open Economy Macroeconomics: A Survey,” Journal of International Economics, 54, pp. 235-266.

Laxton, Douglas, and Paolo Pesenti, 2003, "Monetary Rules for Small, Open, Emerging Economics," Journal of Monetary Economics, 49, pp. 1109-46.

Leitemo, Kai, and Ulf Soderstrom, 2005, "Simple Monetary Policy Rules and Exchange Rate Uncertainty," Journal of International Money and Finance, 24(3), pp. 481507.

Lucas, R. E. Jr., 1972, "Expectations and the Neutrality of Money," Journal of Economic Theory, 4, pp. 103-24. 
Lucas, R. E. Jr., 1973, "Some International Evidence on Output Inflation Tradeoff," American Economic Review, 63, pp. 326-35,

Malik, Hamza Ali, 2005, "Monetary-Exchange Rate Policy and Current Account Dynamics," Lakehead University, Canada, manuscript.

Martins, J., Scarpetta, S., Pilat, D., 1996, "Mark-up Pricing, Market Structure and the Business Cycle", OECD Economic Studies 27 (II), pp. 71-106.

McCallum, Bennett T., 1983, "On Non-uniqueness in Rational Expectations Models: An Attempt at Perspective." Journal of Monetary Economics, 11, pp. 139-168.

McCallum, Bennett T., 1998, "Solutions to Linear Rational Expectations Models: A Compact Exposition." Economics Letters, 61, pp. 143-147.

McCallum, Bennett T. and Edward Nelson, 1999, "An Optimizing IS-LM Specification for Monetary Policy and Business Cycle Analysis," Journal of Money, Credit, and Banking, 31(3), pp. 296-316.

McCallum, Bennett T. and Edward Nelson, 1999, "Performance of Operational Policy Rules in an Estimated Semiclassical Structural Model.” In John Taylor ed., 1999, Monetary Policy Rules. Chicago: The University of Chicago Press.

McCallum, Bennett T. and Edward Nelson, 2001, "Monetary Policy for an Open Economy: An Alternative Framework with Optimizing Agents and Sticky Prices.” Oxford Review of Economic Policy, 16, pp. 74-91.

Miranda, Mario J., and Paul L. Fackler, 2002, Applied Computational Economics and Finance. The MIT Press, Cambridge, Massachusetts.

Monacelli, Tommaso, 2003, "Monetary Policy in A Low Pass-Through Environment," European Central Bank, Working Paper, No. 227. 
Morrison, C., 1994, "The Cyclical Nature of Markups in Canadian Manufacturing: A Production Theory Approach", Journal of Applied Econometrics 9 (3), pp. 269282.

Obstfeld, Maurice and Kenneth Rogoff, 1995a, "Exchange Rate Dynamics Redux," Journal of Political Economy, 103, pp. 624-660.

Obstfeld, Maurice and Kenneth Rogoff, 1995b, "The Intertemporal Approach to the Current Account," Handbook of International Economics, Vol. III, pp. 17311799 .

Obstfeld, Maurice and Kenneth Rogoff, 1996, Foundations of International Macroeconomics, Cambridge, Massachusetts: The MIT Press.

Orphanides, Athansios, 2000, "The Quest for Prosperity without Inflation," Working Paper No. 15, European Central Bank.

Orphanides, Athansios and John C. Williams, 2005a, "Inflation Targeting under Imperfect Knowledge." Federal Reserve Board, Washington D.C., mimeo.

Orphanides, Athansios and John C. Williams, 2005b, "Robust Monetary Policy with Imperfect Knowledge." Federal Reserve Board, Washington D.C., mimeo.

Pontryagin, L. S., S. V. Boltyanskii, R. V. Gamkrelidze, and E. F. Mishchenko, 1962, The Mathematical Theory of Optimal Processes, New York, Wiley.

Rao, C.Radhakrishna and M. Bhaskara Rao, 1998, Matrix Algebra and Its Applications to Statistics and Econometrics. World Scientific Publishing Co. Pte. Ltd., Singapore. Rogers, John H., and Michael Jenkins, 1996, "Haircuts or Hysteresis? Sources of Movements in Real Exchange Rates," Journal of International Economics, 38, pp. $339-360$. 
Rogoff, Kenneth, 1996, "The Purchasing Power Parity Puzzle," Journal of Economic Literature, 34, pp. 647-668.

Rotemberg, Julio J. and Michael Woodford, 1999, "Interest-Rate Rules in an Estimated Sticky-Price Model," in John B. Taylor, ed., "Monetary Policy Rules." Chicago: University of Chicago Press.

Rotemberg, Julio J. and Michael Woodford, 1995, "Dynamic General Equilibrium Models with Imperfectly Competitive Product Markets," in T. F. Cooley (ed.), Frontiers of Business Cycle Research, Princeton University Press, Princeton, pp. 243-293.

Rotemburg, Julio J., 1982, "Sticky Prices in the United States," Journal of Political Economy, 90, pp. 1187-1211.

Rotemberg, Julio J. and Michael Woodford, 1997, “An Optimization-Based Econometric Framework for the Evaluation of Monetary Policy:" NBER Macroeconomics Annual, 1997, 12, pp. 297-346.

Rudebusch, Glenn D. and Lars E. O. Svensson, 1999, "Policy Rules for Inflation Targeting", in John Taylor (ed.), "Monetary Policy Rules", Chicago, University of Chicago Press, pp. 203-262.

Rudebusch, Glenn D., 2002, "Term Structure Evidence on Interest Rate Smoothing and Monetary Policy Inertia,” Journal of Monetary Economics, 49, pp. 1161-1187.

Sargent, Thomas J., 1987, Macroeconomic Theory, Academic Press, Inc., California.

Schott, James R., 1997, Matrix Analysis for Statistics, John Wiley \& Sons, INC.

Simon, Carl P. and Lawrence Blume, 1994, Mathematics for Economists, W. W. Norton \& Company, Inc. New York. 
Sims, Christopher A. 2001, "Solving Linear Rational Expectations Models." Computational Economics, 20, pp. 1-20.

Smets, Frank, and Raf Wouters, 2000, "Optimal Monetary Policy in a Small Open Economy: An Application to the Euro Area," European Central Bank, manuscript.

Smets, Frank and Raf Wouters, 2002, "Openness, Imperfect Exchange Rate PassThrough and Monetary Policy." Journal of Monetary Economics, 49, pp. 947981.

Smets, Frank, and Raf Wouters, 2002, "An Estimated Dynamic Stochastic General Equilibrium Model of the Euro Area," European Central Bank, May, manuscript.

Soderlind, Paul, 1999, "Solution and Estimation of RE Macromodels with Optimal Policy." European Economics Review, 43, pp. 813-823.

Steinsson, Jon, 2002, "Optimal Monetary Policy in an Economy with Inflation Persistence," Harvard University, May, manuscript.

Steinsson Jon, 2003, "Optimal Monetary Policy in an Economy with Inflation Persistence." Journal of Monetary Economics, 50, pp. 1425-1456.

Sutherland, Alan, 2002, "Cost Push Shocks and the Optimal Choice of Monetary Target in an Open Economy", Discussion paper 27/02, Economic Research Centre of the Deutsche Bundesbank.

Sutherland, Alan, 2005, "Incomplete Pass-Through and the Welfare Effects of Exchange Rate Variability," Journal of International Economics, 65, pp. 375-399.

Svensson, Lars E. O., 1997, "Inflation Targeting: Some Extensions", NBER working paper 5962. 
Svensson, Lars E. O. and Michael Woodford, 1999, "Implementing Optimal Policy Through Inflation-Forecast Targeting", Princeton University, November.

Svensson, Lars E. O., 2000, "Open Economy Inflation Targeting," Journal of International Economics, 50, pp. 155-183.

Svensson, Lars E.O. and Michael Woodford, 2003, "Indicator Variables for Optimal Policy," Journal of Monetary Economics 50, pp. 691-720

Svensson, Lars E.O. and Michael Woodford, 2004, "Indicator Variables for Optimal Policy under Asymmetric Information," Journal of Economic Dynamics \& Control 28 pp. $661-690$.

Taylor, John, 1993, "Discretion versus Policy Rules in Practice," Carnegie-Rochester Conference Series on Public Policy, 39: pp195-214.

Taylor, John B., 1999a, "Robustness and Efficiency of Monetary Policy Rules as Guidelines for Interest Rate Setting by the European Central Bank," Journal of Monetary Economics, 43(3), pp. 655-679.

Taylor, John B., (ed.), 1999b, Monetary Policy Rules, Chicago: The University of Chicago Press.

Uhlig, Harald, 1999, "A Toolkit for Analyzing Nonlinear Dynamic Stochastic Models Easily." In Ramon Marimon and Andrew Scott, (ed.), Computational Methods for the Study of Dynamic Economic, Oxford University Press, Oxford.

Walsh, Carl E., 2003, Monetary Theory and Policy, second edition, The MIT Press, Cambridge.

Weerapana, A. 2000, "The Performance of Simple Monetary Policy Rules in a Large Open Economy," Wellesley College working Paper, No, 2000-05. 
Whiteman, Charles H., 1983, Linear Rational Expectations Models: A User's Guide. University of Minnesota Press, Minneapolis.

Wicksell, Knut, 1898, Interest and Rate, 1898, English translation by R. F. Kahn, London: Macmillan, for the Royal Economic Society, 1936. Reprinted New York: Augustus M. Kelly, 1962.

Wicksell, Knut, 1907, "The Influence of the Rate of Interest Rate on Prices," Economic Journal, 17: pp 213-220.

Woodford, Michael, 1996, "Control of the public debt: A Requirement for Price Stability?" Mimeo, Princeton University.

Woodford, Michael, 1999, "Optimal Monetary Policy Inertia," NBER Working Paper No. 7261.

Woodford, Michael, 2001a, "The Taylor Rule and Optimal Inertia." American Economic Review Papers and Proceedings, 91, pp. 232-237.

Woodford, Michael, 2001b, "Inflation Stabilization and Welfare," NBER Working Paper, No. 8071 .

Woodford Michael, 2003 Interest and Prices: Foundations of a Theory of Monetary Policy Princeton, N.J., Princeton University Press, Princeton and Oxford.

Yun, Tack, 1996, "Nominal Price Rigidity, Money Supply Endogeneity, and Business Cycles." Journal of Monetary Economics, Vol. 37, pp. 345-370. 